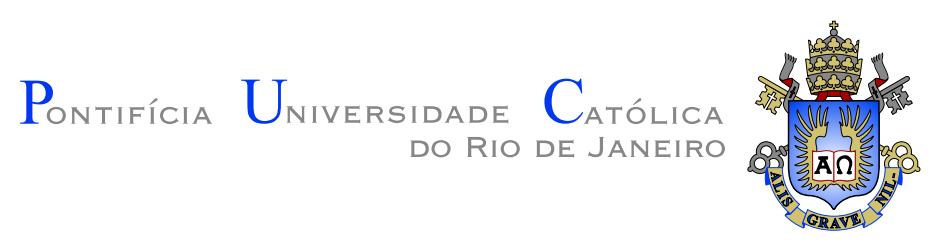

Thiago Mühlbeier

\title{
Potencial de um detector de neutrinos no laboratório subterrâneo de ANDES para o estudo de neutrinos de supernovas
}

\begin{abstract}
Tese de Doutorado
Tese apresentada ao Programa de Pós-graduação em Física do Departamento de Física da PUC-Rio como requisito parcial para obtenção do título de Doutor em Física
\end{abstract}

Orientador: Prof. Hiroshi Nunokawa

Rio de Janeiro Abril de 2013 


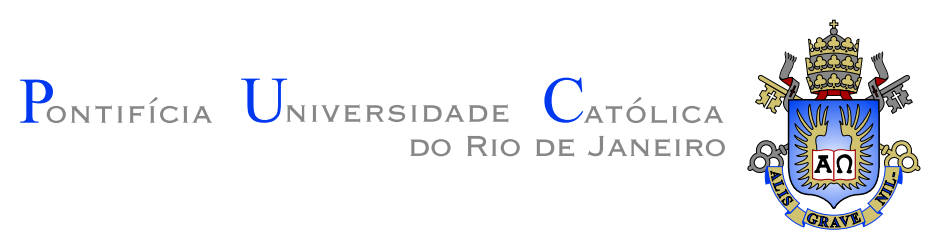

Thiago Mühlbeier

\title{
Potencial de um detector de neutrinos no laboratório subterrâneo de ANDES para o estudo de neutrinos de supernovas
}

\begin{abstract}
Tese apresentada ao Programa de Pós-graduação em Física do Departamento de Física do Centro Técnico Científico da PUC-Rio como requisito parcial para obtenção Do título de Doutor em Física. Aprovada pela Comissão Examinadora abaixo assinada.
\end{abstract}

\author{
Prof. Hiroshi Nunokawa \\ Orientador \\ Departamento de Física - PUC-Rio \\ Prof. Edivaldo Moura Santos \\ UFRJ \\ Prof. Enio Frota da Silveira \\ Departamento de Física - PUC-Rio \\ Prof. Ernesto Kemp \\ UNICAMP
}

Prof. Carla Brenda Bonifazi

UFRJ

Prof. João Carlos Costa dos Anjos

CBPF

Prof. José Eugenio Leal

Coordenador Setorial do Centro Técnico Científico - PUC-Rio

Rio de Janeiro, 30 de Abril de 2013 
Todos os direitos reservados. É proibida a reprodução total ou parcial do trabalho sem autorização da universidade, do autor e do orientador.

\section{Thiago Mühlbeier}

Graduou-se em Licenciatura Plena e Bacharelado em Física na Universidade Estadual do Rio de Janeiro em 2006. Em 2009 obteve o grau de Mestre em Ciências - Física pela Pontifícia Universidade Católica do Rio de Janeiro.

Ficha Catalográfica

Mühlbeier, Thiago

Potencial de um detector de neutrinos no laboratório subterrâneo de ANDES para o estudo de neutrinos de supernovas / Thiago Mühlbeier; orientador: Hiroshi Nunokawa. - Rio de Janeiro : PUC-Rio, Departamento de Física, 2013.

v., 149 f: il. ; $29,7 \mathrm{~cm}$

1. Tese (doutorado) - Pontifícia Universidade Católica do Rio de Janeiro, Departamento de Física.

Inclui referências bibliográficas.

1. Física - Tese. 2. Neutrinos de Supernovas. 3. Triangulação. 4. Oscilação de Neutrinos. 5. Laboratórios Subterrâneos. 6. ANDES. 7. Efeito de Matéria da Terra. 8. Efeito MSW. I. Nunokawa, Hiroshi. II. Pontifícia Universidade Católica do Rio de Janeiro. Departamento de Física. III. Título.

CDD: 510 


\section{Agradecimentos}

Ao meu orientador, Prof. Dr. Hiroshi Nunokawa, pela paciência, dedicação e as valiosas dicas de como resolver os infindáveis problemas surgidos no decorrer desta tese;

Aos meus pais, Ervino e Lília, por sempre terem acreditado em mim e me incentivado nos estudos;

À minha namorada, Kelly, pela paciência, compreensão e apoio constante durante os últimos meses do meu doutorado.

À minha irmã Cláudia, meu cunhado, Ivo, e meus sobrinhos Rafael e Nicole pelo apoio e incentivo das mais diversas formas;

Ao meu irmão, Marcos, minha cunhada Adriane e meus sobrinhos Matheus, Karla e Maria Eduarda que, mesmo distantes, apoiaram, de uma forma ou de outra, o meu trabalho;

Aos meus parentes, sejam primos, tios ou tias pela convivência que tivemos e os ensinamentos que obtive deste contato;

Aos meus professores do ensino fundamental e médio, no Instituto Sinodal da Paz, que plantaram a semente da curiosidade em mim, em especial aos professores Blázio, Alceu, Marlise e Gisela;

Aos meus professores da graduação, na UERJ, e aos da pós-graduação, da PUC-Rio, que foram responsáveis pela minha formação acadêmica;

Aos meus amigos de graduação, pelos ótimos momentos que passamos juntos, seja estudando ou nos divertindo em algum churrasco;

Aos meus amigos e colegas de trabalho da área de neutrinos, Fábio e Alexander, pelas discussões que sanaram inúmeras dúvidas, tanto em física como em fortran ou gnuplot, evitando que eu assumisse conceitos errôneos ou cometesse erros primários;

Aos demais alunos e funcionários da Pós-Graduação do Departamento de Física da PUC-Rio que, de incontáveis formas, ajudaram no andamento desta tese, em especial ao Jefferson, Vanessa, Mari, Giza, Márcia, Eliane e Julinho;

Ao CNPq e à FAPERJ pelo apoio financeiro, que foi de fundamental importância. 


\section{Resumo}

Mühlbeier, Thiago; Nunokawa, Hiroshi. Potencial de um detector de neutrinos no laboratório subterrâneo de ANDES para o estudo de neutrinos de supernovas. Rio de Janeiro, 2013. 149p. Tese de Doutorado - Departamento de Física, Pontifícia Universidade Católica do Rio de Janeiro.

A oportunidade ímpar da construção do primeiro laboratório subterrâneo no Hemisfério Sul precisa ser alicerçada com importantes objetivos científicos, de maneira a competir com os demais laboratórios já existentes ao redor do planeta. Assim, no intuito de auxiliar na solidificação desta ideia, esta tese tem o propósito de verificar o potencial de um detector de neutrinos no futuro laboratório de ANDES para a observação de neutrinos vindos da próxima Supernova Galáctica. A observação de tais partículas é de fundamental importância para a compreensão do mecanismo de explosão de estrelas e para inferir ou desvendar propriedades dos neutrinos. Para tal, simulamos o número de eventos para o decaimento $\beta$ inverso e para o espalhamento elástico $\nu-p$ que seriam obtidos com a próxima supernova com o detector de ANDES considerando diferentes tipos de cintiladores líquidos, levando em conta o efeito MSW. Mostramos que através do espalhamento elástico $\nu-p$ é possível reconstruir o fluxo total de todos os sabores de neutrinos, energia média e total liberados por neutrinos não-eletrônicos. Além disso, a comparação da razão dos números de eventos que vem de corrente carregada (depende de oscilação) e de corrente neutra (independente de oscilação), nos permite inferir a hierarquia de massa para alguns casos. Adicionalmente, estudamos a contribuição do laboratório de ANDES para a observação do efeito de matéria da Terra ao compararmos dados com outros detectores do Hemisfério Norte. Finalmente, investigamos a precisão angular para determinar a direção de uma supernova apenas usando o registro de neutrinos, que pode ser o caso de Supernovas que formam buracos negros ou sofram efeito de obscurecimento devido a poeira interestelar.

\section{Palavras-chave}

Neutrinos de Supernovas; Triangulação; Oscilação de Neutrinos; Laboratórios Subterrâneos; ANDES; Efeito de Matéria da Terra; Efeito MSW; 


\section{Abstract}

Mühlbeier, Thiago; Nunokawa, Hiroshi (Advisor). Potential of a neutrino detector at the ANDES underground laboratory for the study of supernova neutrinos. Rio de Janeiro, 2013. 149p. PhD Thesis - Departamento de Física, Pontifícia Universidade Católica do Rio de Janeiro.

The unique opportunity of construction of the first underground laboratory in the Southern Hemisphere must be grounded with important scientific goals in order to compete with others already existing laboratories around the world. In order to assist this idea as much as possible, this $\mathrm{PhD}$ Thesis aims to investigate the potential of a future neutrino detector at the ANDES laboratory to the observation of neutrinos coming from the next Galactic Supernova. The observation of such particles is of fundamental importance to understanding the mechanism and starburst inferred or unravel properties of neutrinos as mass hierarchy, for example. To this end, we simulate the number of events for inverse $\beta$ decay and the elastic scattering of $\nu-p$ that would be obtained with nearby supernova at the ANDES detector taking into account different types of liquid scintillator and the phenomenon of MSW effect. We show that through the $\nu-p$ elastic scattering is possible to reconstruct the total flux of all flavors of neutrinos, total and average energy released by non-electron neutrinos. Furthermore, comparison of the ratio of observed and theoretical prediction, the number of events coming from charged current (which depends oscillation) and neutral current (independent of oscillation), allows us to infer mass hierarchy at some cases. We also studied the contribution of the ANDES laboratory to the observation of the Earth matter effect combining data with other detectors in the Northern Hemisphere. Finally, we investigate the angular precision for determining the direction of the supernova only using its neutrinos, which can be the situation when either supernovae collapses forming black holes or obscuration due to interstellar dust.

\section{Keywords}

Supernovae Neutrinos; Underground Laboratories; Neutrino Oscillation; ANDES; MSW Effect; Earth Matter Effect; Triangulation Technique; 


\section{Sumário}

1 Introdução 15

2 Teoria das Supernovas $\quad 20$

2.1 Um Pouco de História 20

2.2 Evolução Estelar 21

2.3 Tipos de Supernovas 28

2.4 Etapas da Explosão de uma Supernova (SN) 33

2.5 Espectro dos Neutrinos Provenientes de SN 44

2.6 Modelo de Distribuição de SN na Galáxia 45

$\begin{array}{lll}2.7 & \text { SN1987A } & 46\end{array}$

3 Oscilações de Neutrinos $\quad 50$

3.1 Neutrinos 50

3.2 Oscilação no Vácuo 52

3.3 Mistura para Duas Gerações 60

3.4 Oscilação de Neutrinos na Matéria 62

3.5 Evolução dos Estados de Sabor 66

$\begin{array}{lll}3.6 & \text { Efeito MSW } & 68\end{array}$

3.7 Oscilação de Neutrinos em Supernovas 70

3.8 Probabilidade de Oscilação e Fluxo de Neutrinos em Detectores $\quad 77$

3.9 Quantificando o Efeito de Matéria 85

4 Detector de Neutrinos no ANDES $\quad 87$

$\begin{array}{lll}4.1 & \text { Laboratórios Subterrâneos } & 87\end{array}$

4.2 Detectores com Cintilador 94

$\begin{array}{lll}4.3 & \text { Por que ANDES? } & 102\end{array}$

5 Resultados $\quad \mathbf{1 0 6}$

$\begin{array}{lll}5.1 & \text { Metodologia } & 106\end{array}$

5.2 ANDES com Alquilbenzeno 110

5.3 Comparando Eventos de Corrente Carregada e Corrente Neutra 113

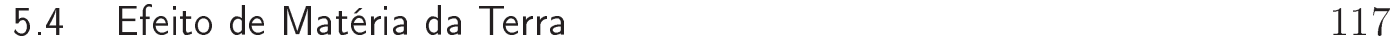

5.5 Triangulação 123

6 Conclusão 133 


\section{Lista de figuras}

1.1 Primeiro experimento para detectar neutrinos, em 1953. Retirado da Ref. [9].

1.2 Panorama da distribuição dos laboratórios subterrâneos atualmente. 19

2.1 Diagrama Hertsprung-Russell. A Sequência Principal é a linha diagonal(na verdade, uma faixa) onde as estrelas permanecem o maior tempo de sua evolução, durante a queima de hidrogênio.

2.2 Diagrama H-R onde é mostrado a luminosidade das estrelas relativas ao Sol em função da temperatura. A Sequência Principal é a faixa onde as estrelas permanecem o maior tempo de sua evolução, durante a transformação do hidrogênio em hélio. Elas ingressam sempre pela parte inferior da faixa. Estrelas de pequena massa na parte inferior, as de massa intermediária na parte central e as de grande massa na parte superior da sequência principal.

2.3 Camadas da queima nuclear de uma estrela muito massiva ao final de sua evolução. Figura de Mafalda Martins, ESO.

2.4 Curva de luz de SNe dos Tipos I e II.

2.5 Quadro de classificação de Supernovas. Reproduzido da Ref. [38].

2.6 Tipos de SN de estrelas massivas em função da massa e metalicidade iniciais.

2.7 Estrutura de uma estrela de $15 \mathrm{M}_{\odot}$ antes de colapsar. Superior: dentro do núcleo a emissão de neutrinos $\left(\epsilon_{\nu}\right)$, gerados via captura eletrônica, responde por quase a totalidade da perda de energia da estrela $\left(L_{t o t}\right)$. A energia gerada pela queima dos elementos pode ser observada pelos picos na taxa de geração de energia nuclear $\left(\epsilon_{\text {nuc }}\right)$. Inferior: a distribuição dos elementos químicos dentro de uma estrela de $15 M_{\odot}$. No núcleo, "Fe" inclui todos os isótopos entre $48 \lesssim A \lesssim 65$. Figura retirada da Ref. [47].

2.8 Velocidade de queda e velocidade do som em função do raio com densidade central de $10^{12} \mathrm{~g} \mathrm{~cm}^{-3}$ e massa do progenitor em $15 M_{\odot}$. $O$ ponto onde as velocidades se encontram marca o limite entre os dois tipos de colapso que ocorrem no centro da estrela. Este ponto é chamado de ponto sônico.

2.9 Probabilidade de distribuição de SN na Via Láctea. As linhas sólida (vermelha), tracejada (azul) e pontilhada (verde) indicam estrelas de nêutrons, pulsares e SN IA, respectivamente.

2.10 Distribuição de probabilidade de SN em coordenadas equatoriais. Maiores probabilidades "caem" sobre o disco galáctico. Os pontos são as últimas $10 \mathrm{SN}$ galácticas ocorridas na Via Láctea e a SN1987A, ocorrida na Grande Nuvem de Magalhães. 
3.1 Ilustração representativa da helicidade dos neutrinos. 51

3.2 Ilustração representativa da hierarquia de massa dos neutrinos. 53

3.3 Relação entre os auto-estados de sabor dos neutrinos $\nu_{e}, \nu_{\mu}$ e $\nu_{\tau}$ e os auto-estados de massa $\nu_{1}, \nu_{2}$ e $\nu_{3}$ em termos dos ângulos de mistura $\theta_{12}, \theta_{13}, \theta_{23}$.

3.4 Diagramas de Feynman representando processos de espalhamento elástico coerente que geram os potenciais $V_{C C}$, através da troca de um bóson $\mathrm{W}$, e $V_{N C}$, pela troca de um bóson $\mathrm{Z}$.

3.5 Ilustração que mostra a ressonância para dois sabores em um meio inomogêneo. As linhas pontilhadas mostram os auto-estados de massa efetiva quando não há mistura e as linhas contínuas indicam a massa efetiva dos auto-estados de sabor quando existe o efeito de mistura. Note que a menor diferença de massa quadrada efetiva se dá quando a ressonância tem o seu valor mínimo.

3.6 Diagramas dos níveis de cruzamento para as hierarquias a) normal e b) invertida. As linhas sólidas mostram a evolução da Hamiltoniana efetiva como função da densidade eletrônica enquanto que as linhas pontilhadas correspondem aos sabores. Densidade negativa é referente ao caso dos antineutrinos.

4.1 Fluxo de múons em função da profundidade em metros de águaequivalente para vários laboratórios subterrâneos e minas ao redor do mundo.

4.2 Desenho esquemático do Laboratório Nacional de Gran Sasso, na Itália.

4.3 Desenho esquemático do Observatório de Kamioka, no Japão, que é administrado pela Universidade de Tóquio, com exceção do experimento KamLAND, que pertence à Universidade de Tohoku.

4.4 Desenho esquemático do laboratório SNOLAB, situado em Sudbury, no Canadá. É o laboratório subterrâneo mais profundo atualmente.

4.5 Local onde será construído o laboratório de ANDES.

4.6 Figura esquemática mostrando a diferença de altitude ao longo dos $14 \mathrm{~km}$ de túnel. A fronteira entre o Chile e a Argentina é o melhor local para a construção do laboratório do ponto de vista de blindagem.

4.7 Design previsto para o laboratório ANDES. Os dois tubos paralelos são os túneis de tráfego. Após, os três átrios e os dois poços, grande e pequeno. O poço maior será usado para instalar um detector a base de líquido cintilador.

4.8 Design do experimento Borexino situado na cavidade C do LNGS.

4.9 Esquete do detector Borexino contendo uma esfera de aço inoxidável, protegida por 1040 toneladas de água pura, para barrar múons atmosféricos. 
4.10 Diagrama esquemático do detector de KamLAND.

4.11 Panorama do laboratório SNO, situado há $\sim 2000 \mathrm{~m}$ de profundidade na mina Creighton, em Sudbury, Canadá. Agora será a casa do experimento $\mathrm{SNO}+$.

4.12 O detector do laboratório SNO localizado na cavidade feita exclusivamente para ele. Toda estrutura será reaproveitada pelo experimento $\mathrm{SNO}+$, que substituirá a água pesada por líquido cintilador como meio para detecção.

4.13 Distribuição do fluxo de geoneutrinos na Terra.

4.14 Fluxo total de geoneutrinos esperados em vários locais da Terra, separando a contribuição do manto e da crosta. O ponto em azul mostra o fluxo esperado para o detector de ANDES após 5 anos de tomada de dados com precisão de 1 e $3 \sigma$ de nível de confiança.

5.1 Fluxos de antineutrinos eletrônicos esperados na Terra para a ocorrência de um SN a $10 \mathrm{kpc}$ de distância. No painel superior, $\left\langle E_{\bar{\nu}_{x}}\right\rangle=18 \mathrm{MeV}$, enquanto que no inferior $\left\langle E_{\bar{\nu}_{x}}\right\rangle=22 \mathrm{MeV}$. Em ambos, $\left\langle E_{\bar{\nu}_{e}}\right\rangle=15 \mathrm{MeV}$.

5.2 Distribuição de energia esperada de eventos de $\bar{\nu}_{e}$ para o detector de ANDES. Supomos $\left\langle E_{\bar{\nu}_{e}}\right\rangle=15 \mathrm{MeV}$ (curvas pontilhadas azuis) e $\left\langle E_{\bar{\nu}_{x}}\right\rangle=18 \mathrm{MeV}$ e $22 \mathrm{MeV}$ para os gráficos superior e inferior, respectivamente (curvas verdes tracejadas).

5.3 Distribuição de eventos em função da energia de extinção do próton para o detector de ANDES.

5.4 Os círculos vermelhos representam a reconstrução do fluxo original dos neutrinos provenientes de uma SN distante $10 \mathrm{kpc}$ da Terra para o detector de ANDES através do espalhamento elástico entre neutrino-próton. A barra de erro é equivalente a $1 \sigma$.

5.5 Sensitividade na determinação da $\left\langle E_{\nu_{x}}\right\rangle$ e da $E_{\nu_{x}}^{\text {Tot }}$ originais mostrados em 1, 2 e 3 níveis de confiança para o detector de ANDES através do número de eventos devido ao espalhamento elástico neutrino-próton. Consideramos 3 diferentes valores de entrada para $\left\langle E_{\nu_{x}}\right\rangle=15,18$ e $21 \mathrm{MeV}$ e a mesma $E_{\nu_{x}}^{T o t}=5 \times 10^{52} \mathrm{erg}$. Os valores de entrada estão indicados com um asterisco na figura.

5.6 A razão $R\left(N_{\bar{\nu}_{e} p} / N_{\nu p}\right)$ em função de $\left\langle E_{\nu_{x}}\right\rangle$ para o detector de ANDES.

5.7 Agora razão $R\left(N_{\bar{\nu}_{e} p} / N_{\nu p}\right)$ em função de $\left\langle E_{\bar{\nu}_{e}}\right\rangle$ para o detector de ANDES.

5.8 A razão $R\left(N_{\bar{\nu}_{e} p} / N_{\nu p}\right)$ em função de $\bar{p}$ para o detector de ANDES considerando $\left\langle E_{\nu_{x}}\right\rangle=18$ e $21 \mathrm{MeV}$.

5.9 A razão $R\left(N_{\bar{\nu}_{e} p} / N_{\nu p}\right)$ em função da razão das luminosidades $L_{\bar{\nu}_{e}} / L_{\nu_{x}}$ para o caso onde $E_{\nu_{x}}=18 \mathrm{MeV}$ e os demais parâmetros são os mesmos da tabela 5.1. 
5.10 Um oscilograma de neutrino onde o isocontorno da razão $P^{\oplus}\left(\bar{\nu}_{1} \rightarrow\right.$ $\left.\bar{\nu}_{e}\right) / c_{12}^{2}$ é feito no plano do ângulo nadiral, $\theta_{\text {nadir }}$, e da energia do neutrino. $O$ valor unitário no gráfico representa a inexistência (ausência) do efeito de matéria da Terra.

5.11 Diferença fracional no espectro do fluxo de neutrinos com e sem o efeito de matéria da Terra, $\Delta F_{\bar{\nu}_{e}} / F_{\bar{\nu}_{e}}$.

5.12 Soluções possíveis para apontar o local de ocorrência de uma SN que seja consistente com a diferença de tempo de chegada dado pela combinação de detectores organizados par a par. Coordenada correta para este exemplo é $\alpha=17^{h} 42^{m} 27^{s}$ e $\delta=-28^{\circ} 55^{\prime}$.

5.13 Situação onde os quatro detectores utilizados nesta tese são agrupados três a três. Cada linha é uma combinação diferente. As cores roxo, amarelo e verde indicam, respectivamente, níveis de confiança de $1 \sigma, 2 \sigma$ e $3 \sigma$. Supomos que a incerteza na medida da diferença temporal entre dois detectores é $\pm 4 \mathrm{~ms}$ para a coluna da esquerda e \pm 2 ms para a da direita.

5.14 Situação onde os quatro detectores utilizados nesta tese são agrupados três a três. Cada linha é uma combinação diferente. As cores roxo, amarelo e verde indicam, respectivamente, níveis de confiança de $1 \sigma, 2 \sigma$ e $3 \sigma$. Supomos que a incerteza na medida da diferença temporal entre dois detectores é $\pm 4 \mathrm{~ms}$ para a coluna da esquerda e \pm 2 ms para a da direita.

5.15 Idem a Fig. 5.15, mas para a explosão de SN localizada na posição oposta ao centro da Via Láctea. 


\section{Lista de tabelas}

2.1 Eventos de neutrinos detectados nos experimentos Kamiokande-II, IMB e Baksan relativos a SN1987A. A primeira coluna indica a ordem dos eventos, a segunda coluna mostra o tempo de detecção, considerando o primeiro evento como tempo zero. A terceira informa-nos a energia do neutrino incidente e a quarta coluna nos diz a incerteza para a energia e, finalmente, a última coluna fornece a taxa média de ruído para cada evento.

3.1 Tabela de léptons e anti-léptons

3.2 Valores de acoplamento para campos de férmions. $\mathrm{E} \sin ^{2} \theta_{w}=$ 0.231 .

4.1 Número esperado de eventos de geoneutrinos para o detector de ANDES com 3 kt de líquido cintilador após um ano de operação com $80 \%$ de eficiência considerando diferentes lugares na Terra. TNU significa terrestrial neutrino units.

5.1 Parâmetros de referência de SN usados ao longo deste trabalho, a menos que explicitamente declarado diferente.

5.2 Número esperado de eventos de neutrinos de SN observados através do decaimento beta inverso e pelo espalhamento elástico prótonneutrino para os 3 tipos de líquidos cintiladores com três kt e uma SN distante $10 \mathrm{kpc}$ da Terra. Eles são (a) $80 \%$ de $\mathrm{C}_{12} \mathrm{H}_{26}$ e $20 \%$ de $\mathrm{C}_{9} \mathrm{H}_{12}$ usado em KamLAND, (b) $\mathrm{C}_{9} \mathrm{H}_{12}$ (pseudocumeno) usado no Borexino, e (c) $\mathrm{C}_{6} \mathrm{H}_{5} \mathrm{C}_{12} \mathrm{H}_{25}$ (alquilbenzeno) para ser usado no detector $\mathrm{SNO}+\mathrm{NH}$ e $\mathrm{IH}$ indicam, respectivamente, hierarquias normal e invertida. Para $\nu+p \rightarrow \nu+p$ nós consideramos a energia cinética devido ao recuo do próton, $T_{\text {que }}$, com energia superior a $0,2 \mathrm{MeV}$, de acordo com as referências citadas no texto.

5.3 Precisão mostrada de acordo com nível de confiança para a determinação da $\left\langle E_{\nu_{x}}\right\rangle$ e da $E_{\nu_{x}}^{\text {Tot }}$ para o detector de ANDES devido à observação de uma SN conforme parâmetros da tabela 5.1. Os demais parâmetros foram mantido fixos. Na primeira coluna estão representados os valores de entrada.

5.4 Coordenadas geográficas dos detectores considerados nesta tese. $\mathrm{Na}$ última coluna mostramos a probabilidade de neutrinos oriundos de uma SN galáctica cruzarem o interior da Terra, sendo através do Manto ou do conjunto Manto + Núcleo (indicado apenas como Núcleo). 
5.5 Probabilidade de sombreamento da Terra para a associação de dois detectores localizados em Kamioka, no Japão, e no Polo Sul.

5.6 Probabilidade de sombreamento pela Terra para o caso onde temos três detectores combinados: Super-Kamiokande, IceCube e ANDES.

5.7 Probabilidade de sombreamento da Terra para o caso com quatro detectores: Kamioka, Polo Sul, ANDES e Sudbury.

5.8 Número de eventos esperados de decaimento $\beta$ inverso no detector de Super-Kamiokande $\left(1.7 \times 10^{33}\right.$ prótons livres $)$ para uma SN distante $5 \mathrm{kpc}$ da Terra para $E<30 \mathrm{MeV}, 30<E / \mathrm{MeV}<40$, $40<E / \mathrm{MeV}<50$ e $E>50 \mathrm{MeV}$ para o caso de vácuo e efeito de matéria com uma distância de $1000 \mathrm{~km}$ e diversos parâmetros de SN. Nós supomos $L_{\overline{\nu_{e}}} / L_{\nu_{x}}=1$ and $\left\langle E_{\bar{\nu}_{e}}\right\rangle=15$ $\mathrm{MeV}$, assim como que o detector de Super-Kamiokande recebe neutrinos sem o efeito de matéria da Terra enquanto que o detector de ANDES recebe os neutrinos após os mesmos terem viajado $1000 \mathrm{~km}$ dentro do planeta. Os números na linha indicada como "vácuo" são aqueles a serem observados pelo detector SK, que precisa compará-los com a previsão teórica para o próprio SK, calculada do número de eventos observados no detector de ANDES. Na última coluna nós mostramos em quantos $\sigma$ a observação em 1000 km é distinguível do vácuo, e Comp.é a abreviação para "Compatibilidade". Na primeira coluna, o significa "observado" e p, "previsto".

5.9 Número de eventos estimados par uma SN a 10 kpc da Terra, assim como a precisão na determinação do tempo de chegada dos neutrinos. 128 
"A science is any discipline in which the fool of this generation can go beyond the point reached by the genius of the last generation."

Herman Max Gluckman 


\section{Introdução}

Embora hoje acreditemos que os primeiros neutrinos surgiram logo após o nascimento do universo, há aproximadamente 15 bilhões de anos, e ainda são produzidos em grandes quantidades nos interiores estelares, foi somente no final do séc. XIX, quando Becquerel ${ }^{1}$ e o casal Curie $^{2}$ descobriram a radiatividade a partir de sais de urânio, que foram dados os primeiros passos, embora ainda bem distantes, em direção ao conhecimento de sua existência.

Rutherford $^{3}$, antes da virada do século, mostrou a existência de 2 tipos de radiação ${ }^{4}, \alpha$ e $\beta$. Os primeiros anos do séc. XX tornaram-se uma verdadeira corrida na busca de melhor compreensão dos tipos de radiação, sendo realizados diversos experimentos por inúmeros físicos, até ser de consenso acadêmico que:

1. Radiação Alfa era composta de núcleos de ${ }^{4} \mathrm{He}$;

2. Radiação Beta eram elétrons gerados no núcleo radiativo; e

3. Radiação Gama era um fóton criado pelo núcleo radiativo com energia de alguns poucos $\mathrm{MeV}$.

Assim, acreditou-se que a radiação beta deveria ter seu espectro de energia discreto por consistir de uma única partícula emitida do núcleo radiativo. Entretanto, Meitner, Hahn, Wilson, von Baeyer e Chadwick, na década de 1910, mostraram que o espectro da radiação beta era contínuo [1, 2, 3, 4].

${ }^{1}$ Antoine Henri Becquerel (1852-1908) foi um físico francês laureado com o Prêmio Nobel de Física pela descoberta da radiatividade ao detectar radiação proveniente do urânio.

${ }^{2}$ Pierre (1859-1906), francês, e Marie Curie (1867-1934), polonesa, foram físicos que tornaram-se conhecidos no meio científico ao conseguirem isolar o elemento rádio, muito mais radiativo que o urânio e também foram agraciados com o Prêmio Nobel de Física de 1903.

${ }^{3}$ Ernest Rutherford (1871-1937) foi um físico neozelandês que, entre inúmeros trabalhos, ficou famoso ao descobrir que a carga de um átomo está concentrada em seu centro, e também desenvolveu a moderna concepção de átomo.

${ }^{4} \mathrm{~A}$ radiação gama $(\gamma)$ foi descoberta em 1901 por Villard. 
Este resultado, usando técnicas mais modernas, foi confirmado em 1927 por Ellis e Wooster [5]. A comprovação do espectro contínuo reforçou a ideia já defendida por Bohr [6], entre outros - da não conservação da energia e do momento angular.

Entretanto, por sua crença incondicional nas leis de conservação, o físico Wolfgang Pauli ${ }^{5}$, sempre rejeitou a ideia de Bohr e isto o levou a prever, em 1930, a existência de uma partícula, que ele chamou de nêutron, cujas características eram: tipo fermiônica, neutra e que interagiria muito fracamente. Esta partícula também seria emitida no decaimento beta, mantendo assim a energia e momento conservados. A predição da existência do neutrino está em uma carta aberta para "Liebe Radiaktive Damen und Herren" ${ }^{6}$ escrita por Pauli para o encontro regional de Tübingen, na Áustria, que ele não pôde comparecer [7].

Em 1932, Chadwick descobriu o nêutron conhecido atualmente [8] e o nome da misteriosa partícula de Pauli foi renomeada, por Fermi ${ }^{7}$, para neutrino, que em italiano significa pequeno nêutron. Fermi formulou a teoria do decaimento beta via interação fraca, em 1934, onde a existência de um neutrino se encaixava muito bem, mas até o início da década de 50 tudo que se tinha eram os resultados, consistentes com a existência do neutrino, das medidas de recúo do núcleo durante o decaimento beta. Somente em 1952 é que Reines $^{8}$ e Cowan ${ }^{9}$ procuraram um modo de medir o decaimento beta através da detecção de um pósitron (descoberto em 1933 por Anderson ${ }^{10}$ ) criado por um antineutrino. O experimento proposto foi de colocar um detector, composto de 1,4 mil litros de cintiladores líquidos, próximo de um reator nuclear em Hanford, Washington, no EUA (ver Fig. 1.1 [9]). Este foi o primeiro experimento de neutrinos produzidos em reatores e usava a reação

${ }^{5}$ Wolfgang Ernst Pauli (1900-1958) foi um físico austríaco que, dentre outras importantes contribuições, postulou o princípio de exclusão dos férmions que leva seu nome, recebendo por isso o Prêmio Nobel de Física de 1945.

${ }^{6}$ Prezados Senhores e Senhoras Radioativos

${ }^{7}$ Enrico Fermi (1901-1954) foi um físico italiano que se destacou pelo desenvolvimento da teoria quântica, na física nuclear, de partículas e mecânica estatística. Recebeu o Prêmio Nobel de Física em 1938 por seu trabalho em radiatividade induzida.

${ }^{8}$ Frederick Reines (1918-1998) foi um físico norte-americano, laureado com o Prêmio Nobel em 1995 pela detecção do neutrino eletrônico em conjunto com Clyde L. Cowan Jr.

${ }^{9}$ Clyde Lorraine Cowan Jr (1919-1974) foi um engenheiro químico norte-americano cujo principal trabalho foi a deteç̧ão do neutrino eletrônico juntamente com Frederick Reines.

${ }^{10}$ Carl David Anderson (1905-1991) foi um físico norte-americano, cujo principal trabalho foi a descoberta do pósitron, que lhe rendeu o Prêmio Nobel de Física em 1936. 


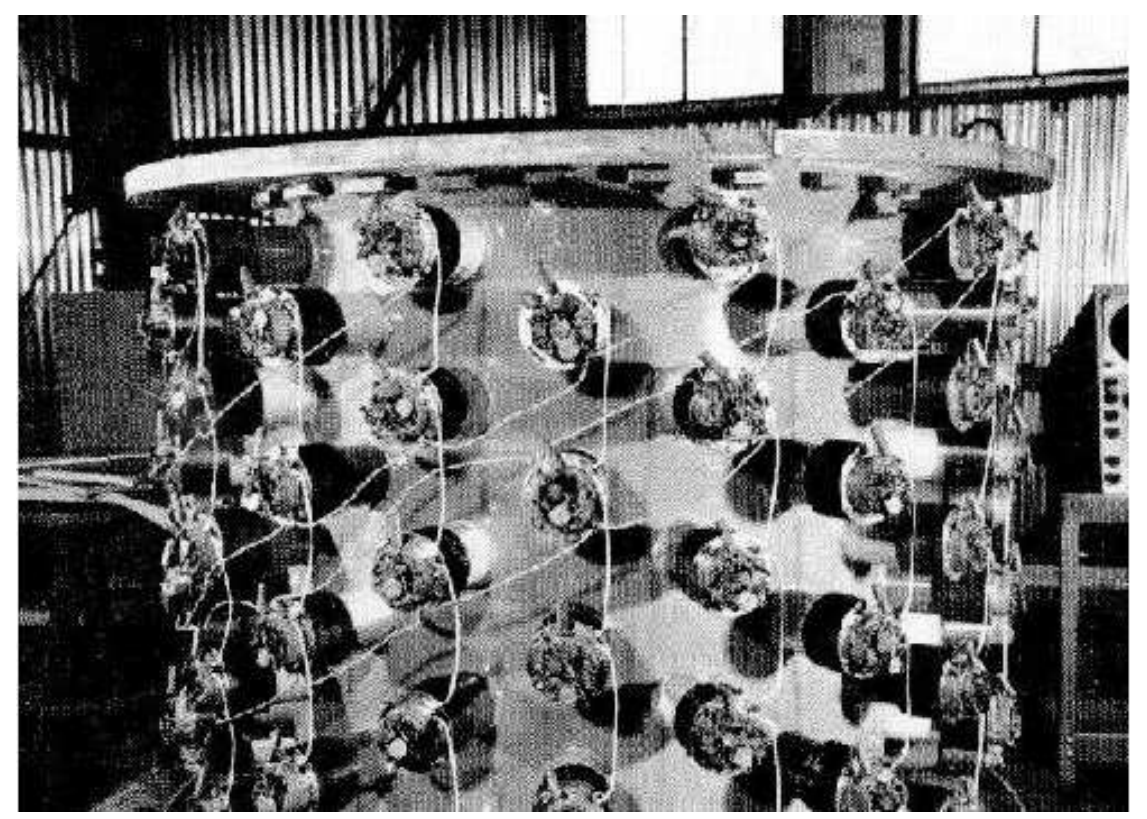

Figura 1.1: Primeiro experimento para detectar neutrinos, em 1953. Retirado da Ref. [9].

$\bar{\nu}+p \rightarrow n+e^{+}$como canal principal.

Os primeiros resultados [10] vieram no verão de 1953, mas não foram convincentes. O experimento foi refeito em 1956 com maiores cuidados, agora na Carolina do Sul e, com as melhorias principalmente com relação ao ruído, foi encontrada a primeira evidência experimental da existência de neutrinos [11]. Com o conceito de número leptônico por sabor conservando-se em interações fracas, introduzido em 1953 por Konopinski e Mahmoud [12] para explicar a não observância de certos modos de decaimento como, por exemplo, $\mu^{-} \rightarrow$ $e^{-}+\gamma$, foi percebido que os neutrinos $\nu_{e}$ e $\nu_{\mu}$ eram partículas distintas. Então, pela reação acima que produz pósitrons, sabemos que a partícula detectada em 1956 foi $\bar{\nu}_{e}$. A procura pelo $\nu_{\mu}$ foi idealizada no começo da década de 60, sendo concretizada em 1962 [13]. Em 1977 Martin Perl [14] descobre a terceira família dos léptons, o tau, indicando que deveria existir o neutrino do tau, mas esta descoberta só apareceu, experimentalmente, nos anos 2000 [15].

Paralelamente às descobertas de várias partículas, começou no final dos anos 60 a funcionar o primeiro detector subterrâneo para neutrinos oriundos de fontes astrofísicas que, à época, significava o Sol. O experimento Homestake [16], localizado na mina de mesmo nome, foi o primeiro do tipo e funcionou por mais de 20 anos, revelando o problema dos neutrinos solares e dando os primeiros indícios de oscilação. Muitos experimentos foram realizados desde então na tentativa de encontrar evidências de oscilação de neutrinos, como o 
Kamiokande [17], no Japão, ou o IMB [18, 19], no EUA. Os resultados obtidos por KamLAND [20] e Super-Kamiokande [21] confirmaram as espectativas a respeito da existência da oscilação de neutrinos, indicando que os neutrinos possuem massa.

Laboratórios subterrâneos tem sido de importância fundamental nos últimos 50 anos e, atualmente, há dezenas deles em operação onde existem os mais variados experimentos, das mais diversas áreas. Entretanto, a física de neutrino continua tendo grande destaque e, mais recentemente, a procura por evidências de matéria escura, já que estas, a princípio, também atuariam apenas via interação fraca.

Curiosamente, todos os laboratórios subterrâneos correntes estão situados no Hemisfério Norte, como mostra a Fig. 1.2. Embora a primeira vista isto não é um problema, já que as partículas estudadas nestes laboratórios cruzam a Terra facilmente, a existência de um laboratório subterrâneo no Hemisfério Sul tem importância científica, devido a vários efeitos. Um exemplo é a modulação do sinal esperado para a matéria escura por causa do movimento de rotação da Terra no halo de matéria escura da Via Láctea. No Hemisfério Sul, a variação no sinal diurno - taxa de matéria escura observada nos experimentos-, comparado com o Hemisfério Norte, é mais acentuado devido a proteção realizada pelo núcleo da Terra em algum momento do dia.

A importância de um laboratório no Hemisfério Sul também tem lugar na física de neutrinos, em especial a astrofísica de neutrinos. Um detector nesta região do globo somado aos já existentes no Hemisfério Norte, por exemplo, permitiria um estudo mais completo do efeito Mikheyev-Smirnov-Wolfenstein (MSW) na Terra, já que por ocasião do surgimento da próxima supernova galáctica a probabilidade de registrarmos eventos vindos diretamente da estrela moribunda e eventos que cruzaram a Terra antes de chegarem ao detector aumentam significativamente [22]. Estes e outros resultados são apresentados nesta tese e estão embasados na Ref. [22].

Assim, com a aprovação da construção do túnel de Água Negra pelos governos da Argentina e do Chile, a comunidade científica da América do Sul viu uma oportunidade ímpar para a construção do primeiro laboratório subterrâneo no Hemisfério Sul.

Esta tese está dividida da seguinte forma: começamos no cap. 2 com uma breve introdução sobre a história de supernovas para depois explicarmos um pouco da física das estrelas, isto é, como elas nascem, crescem e morrem, 


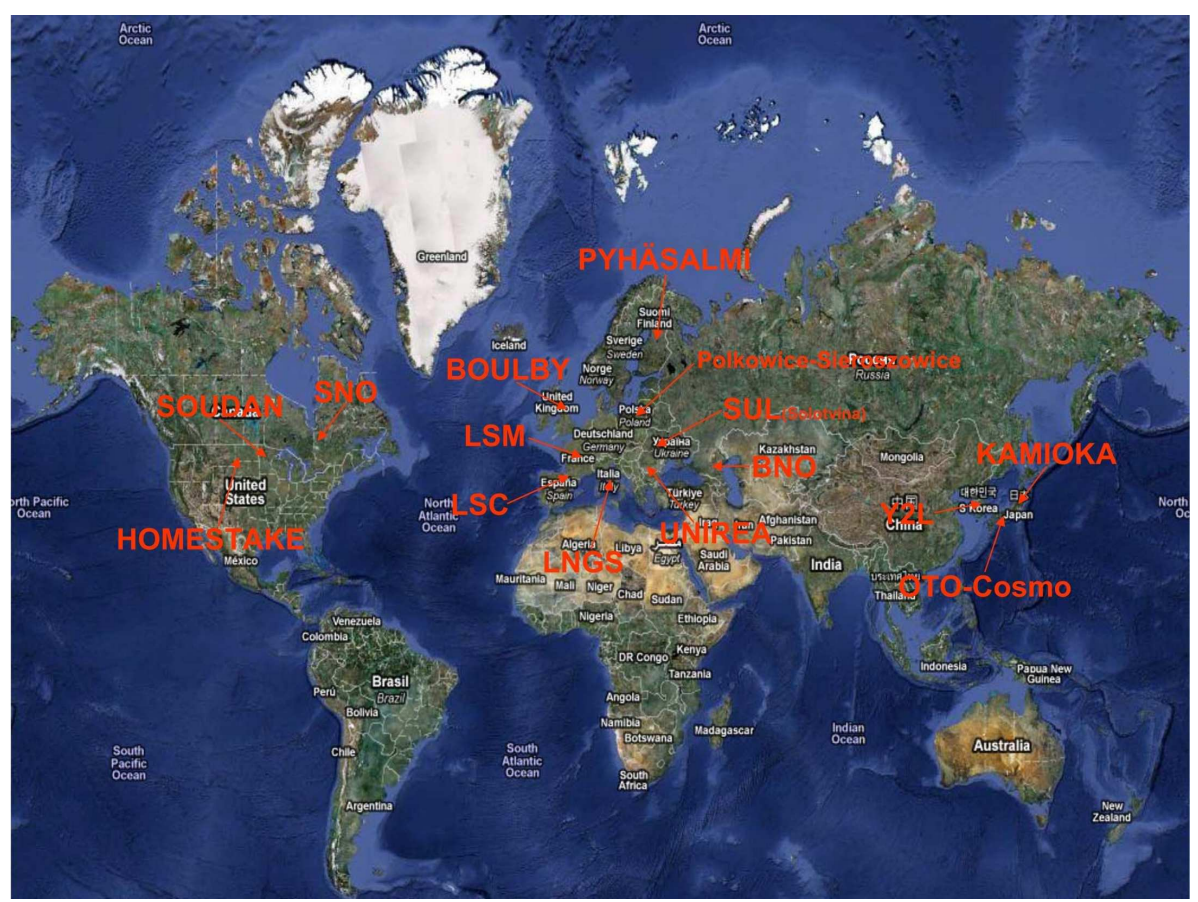

Figura 1.2: Panorama da distribuição dos laboratórios subterrâneos atualmente.

nos aprofundando no processo de explosão para estrelas massivas. No cap. 3 temos a física de partículas: explicamos como se comportam os neutrinos e o processo de oscilação, primeiramente, no vácuo, depois na matéria, que é a parte que nos interessa, pois é no interior das estrelas e da Terra, em regiões de alta e/ou baixa densidades, que ocorrem as oscilações e são alterados os fluxos dos sabores dos neutrinos. Após este primeiro contato com a teoria mais geral, mas importante, vamos ao cap. 4, onde discutiremos um pouco sobre os laboratórios subterrâneos, de modo geral, para depois nos determos a três deles: Gran Sasso, Kamioka e Sudbury. Eles, em especial o detector $\mathrm{SNO}_{+}$, que nortearam este trabalho. Falaremos rapidamente sobre os seus cintiladores e objetivos, assim como resultados alcançados. Finalizando o cap. 4, falaremos com detalhes a respeito do laboratório de ANDES (Água Negra Deep Experiment Site) [23, 24], focando o potencial do laboratório e do detector de neutrinos. O cap. 5 contém os resultados obtidos ao considerarmos o aparecimento de uma supernova galáctica levando-se em conta o detector de ANDES e/ou em combinação com outros detectores em locais distantes para o estudo de propriedades de neutrinos, assim como efeito MSW. As conclusões alcançadas neste estudo estão no cap. 6. 


\section{2}

\section{Teoria das Supernovas}

O capítulo está dividido da seguinte forma: na seção 2.1 veremos um pouco da história das supernovas, os primeiros registros e locais de observação; depois, na seção 2.2, faremos uma abordagem rápida sobre evolução estelar para, na seção 2.3, conhecermos os tipos de classificação de supernovas existentes atualmente. Em seguida, na seção 2.4, vamos nos aprofundar sobre as etapas e os processos de explosões de supernovas. Na seção 2.5 falaremos rapidamente sobre o espectro dos neutrinos, mencionando a função distribuição utilizada neste estudo. Então na seção 2.6 veremos o modelo de distribuição de SN na Galáxia utilizado nesta tese; e fecharemos o capítulo com a seção 2.7 onde descreveremos um pouco sobre o evento que marcou a astrofísica de neutrinos, a observação da SN1987A, os experimentos envolvidos e o que foi registrado.

\section{1}

\section{Um Pouco de História}

No distante século II A.C. astrônomos chineses começaram a registrar com detalhes suas observações astronômicas. Assim, objetos que apareciam no céu repentinamente e permaneciam visíveis por um certo período de tempo (vários meses ou alguns anos) e depois desapareciam foram batizados pelos chineses como "estrelas visitantes". Estas estrelas foram, provavelmente, supernovas [25]. Uma das mais conhecidas foi registrada em 185 D.C. e sua remanescente (RCW 86) produz, ainda hoje, fortes imagens em raio-X [26].

Em maio de 1006 D.C. apareceu a supernova mais brilhante, foi tão intenso que seu brilho foi maior que o de Vênus [27], sendo observada na China, no Oriente Médio e na Europa. Sua remanescente foi observada primeiramente em imagens de rádio, em 1965; e, ultimamente, em raios gama de alta energia [28]. Alguns anos depois, em 1054 surgiu o que hoje conhecemos 
como Nebulosa do Caranguejo, a qual foi novamente vista pelos chineses, mas, desta vez, não há registros da observação na Europa. A SN1054 mostra vários filamentos brilhantes e todo o seu volume é luminoso, o que reforça a hipótese de que há uma estrela de nêutrons em seu centro que emite radiação eletromagnética em todas as frequências com intervalos regulares. Nas SN185 e SN1006 há apenas cascas radiantes, que seria a onda de choque do processo de explosão e não há estrelas de nêutrons em seus centros.

Alguns séculos a frente, temos a observação de uma "nova estrela" realizada pelo jovem físico dinamarquês, Tycho Brahe, em novembro de 1572 . Esta remanescente é observada hoje em raio-X e também não possui estrela de nêutrons em seu interior [29]. Ainda é válido destacar as observações de Kepler, em 1604, de uma supernova que ficou visível por um ano inteiro e hoje é medida em raio-X; assim como a supernova de Cassiopéia A, entre 1650 e 1680, cuja remanescente emite em rádio, as quais foram as últimas supernovas registradas na Galáxia [30, 31].

O termo Supernova (SN) não havia sido utilizado até a década de 30 do século passado. Foram Baade e Zwicky [32] que introduziram o vocábulo para distinguir estas Novas ${ }^{1}$ extremamente luminosas das conhecidas até então. O brilho delas era tão intenso que poderia ser observado mesmo que ocorresse em outra galáxia. Estes dois astrônomos foram os pioneiros no estudo e na busca por SN de maneira mais sistemática, aumentando o número de SN descobertas a cada ano. Até a década de 50, o número de SN observadas anualmente era de algumas unidades. Após este período, a quantidade chegou na casa da dezena e no século 21 temos mais de uma centena de novas SN sendo descobertas anualmente.

\section{2}

\section{Evolução Estelar}

\subsection{1}

\section{Nascimento de uma Estrela}

Há muitas dúvidas sobre o processo exato de formação estelar, mas a hipótese mais aceita é a de aglutinação de partículas por atração gravitaci-

\footnotetext{
${ }^{1}$ Novas são eventos de explosões que resultam em perda de massa da estrela, mas não há mudança em sua estrutura. Também são identificadas através de aumento de brilho e posterior enfraquecimento, mas em proporções muito menores que as supernovas.
} 
onal. Esta concepção é tão antiga quanto a própria ideia de gravidade [33]. Entretanto somente na metade final do século XX que começamos a obter um melhor entendimento sobre a formação de estrelas através da observação de ondas em infravermelho e rádio.

A ação da gravidade é fundamental no processo de formação estelar, mas há outros mecanismos que exercem papel crucial em diferentes escalas ao combater a gravidade. Por exemplo, em dimensões galácticas, a formação de estrelas por aglutinação de partículas em nuvens moleculares, devido a gravidade, é contraposta por forças de marés galácticas ${ }^{2}$, fazendo com que haja a condensação de matéria apenas em áreas onde há uma densidade maior de partículas, sobrepujando este tipo de efeito de maré. Em escalas intermediárias, nas nuvens moleculares gigantes ${ }^{3}$, podem existir turbulência e campos magnéticos exercendo uma função de destaque ao contrapor a ação da gravidade. Finalmente, a pressão térmica é o principal opositor à gravidade em escalas pequenas, isto é, no núcleo de nuvens pré-estelares.

Assim, após a gravidade vencer seus oponentes, irá começar a condensar matéria e após 1000 anos irá formar o que chamamos de protoestrela. Sabemos que $F_{\text {gravitacional }} \propto(1 / \text { distância })^{2}$, o que significa que as regiões mais internas da protoestrela sofrem uma ação maior da gravidade fazendo com que "caiam" mais rapidamente. No início do colapso as partículas não colidem entre si, ou seja, a pressão interna é zero, por isso podemos dizer que o colapso da nuvem se dá em queda livre. Depois de formado o núcleo na região central do colapso, há o período de acreção de partículas das camadas mais externas. Com o aumento das colisões entre as moléculas, os grãos de poeira são aquecidos e irradiam no infravermelho e durante milhares de anos essa radiação consegue escapar e mantém a protoestrela fria, com pressão interna muito pequena. Entretanto, em um dado momento, a densidade no núcleo alcança valores críticos e a própria poeira torna-se opaca para a radiação infravermelha. Isso faz com que a pressão interna e a temperatura aumentem.

Este embate entre pressão e força gravitacional continua até que seja atingido o equilíbrio, chamado de Equilíbrio Hidrostático. A partir daí a

2Maré galáctica é uma força de maré em que objetos "sentem" a ação de um campo gravitacional devido a uma galáxia.

${ }^{3}$ Nuvem molecular gigante é uma espécie de nuvem interestelar composta majoritariamente por hidrogênio, com densidade de $\sim 10^{3}$ partículas por centímetro cúbico e diâmetro que pode chegar a algumas centenas de parsecs $\left(\sim 10^{15} \mathrm{~km}\right)$, onde há a formação de moléculas de $\mathrm{H}_{2}$ e cuja massa varia entre $10^{3}-10^{7}$ massas solares [34]. 


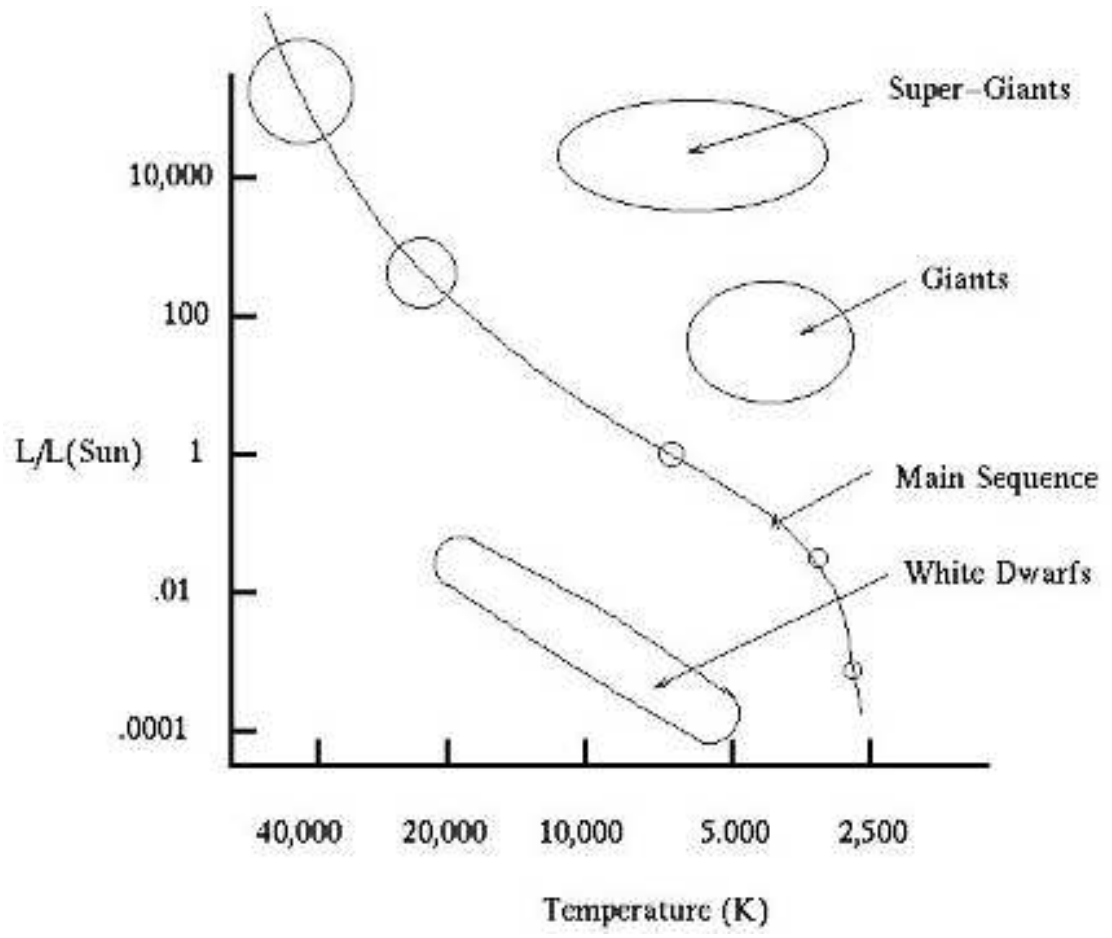

Figura 2.1: Diagrama Hertsprung-Russell. A Sequência Principal é a linha diagonal(na verdade, uma faixa) onde as estrelas permanecem o maior tempo de sua evolução, durante a queima de hidrogênio.

protoestrela passa a chamar-se estrela da pré-sequência principal ou estrela T Tauri e encontra-se localizada no canto direito do diagrama HertzsprungRussell (H-R $)^{4}$, que relaciona a luminosidade das estrelas com suas temperaturas, conforme mostra a Fig. 2.1.

Nesta etapa a estrela da pré-sequência principal possui uma temperatura superficial menor do que terá quando adulta, mas o seu raio e luminosidade, que se deve somente pela irradiação de calor via convecção do núcleo para a superfície, são maiores do que ela terá em seu futuro. Após alguns milhões de anos nesta fase, a parte central da estrela consegue atingir a temperatura de aproximadamente $10^{7} \mathrm{~K}$ e, a esta magnitude, começam a ocorrer as primeiras reações de fusão nuclear do hidrogênio. A partir de então, as fusões nucleares regirão a vida deste astro e ele é considerado oficialmente uma estrela e, daí em diante, pertencente à sequência principal do Diagrama H-R[35].

${ }^{4}$ Trabalho realizado em 1911 pelo astrônomo dinamarquês Ejnar Hertzsprung e em 1914, de maneira independente, pelo astrônomo americano Henry Norris Russell. Ambos observaram que as estrelas não se distribuiam uniformemente, mas se agrupavam em áreas bem delimitadas. 


\section{2 .2}

\section{Sequência Principal}

Estrelas pertencentes à Sequência Principal do Diagrama H-R são consideradas estrelas em fase adulta, isto é, são estrelas que transformam hidrogênio em hélio utilizando um dos dois caminhos possíveis: cadeia próton-próton (pp) ou o ciclo Carbono-Nitrogênio-Oxigênio (CNO). Inicialmente, as estrelas entram na parte inferior da largura da sequência principal, e o local depende de sua massa: estrelas com até 3 massas solares (estrelas de pequena massa) posicionam-se na parte inferior da sequência principal; entre 3 e 10 massas solares (estrelas de massa intermediária) localizam-se na parte central; e acima de 10 massas solares (estrelas de grande massa) ficam na parte superior da sequência principal do Diagrama H-R (Fig. 2.2). O modo de evolução das estrelas depende muito de sua massa e não nos deteremos em nenhum caso específico. Comentaremos as características gerais da evolução para todos os tipos de estrela.

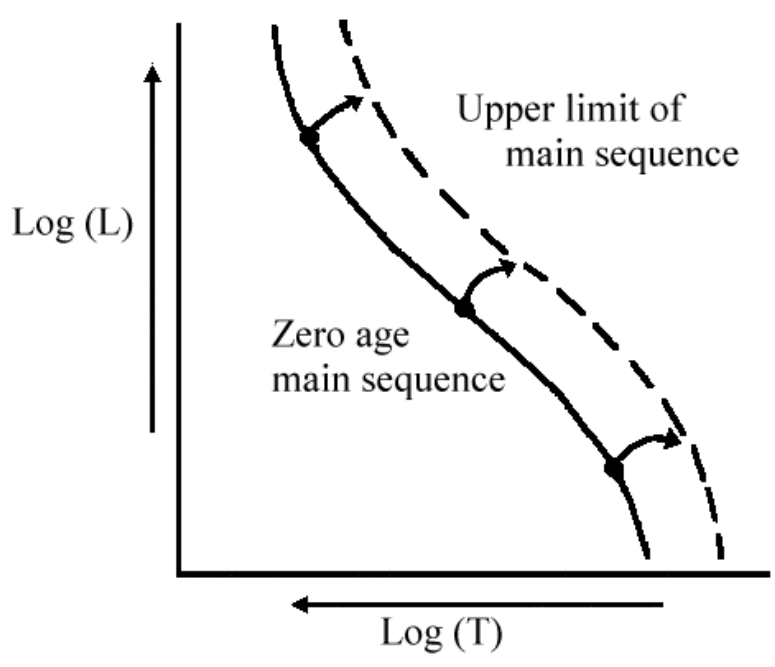

Figura 2.2: Diagrama H-R onde é mostrado a luminosidade das estrelas relativas ao Sol em função da temperatura. A Sequência Principal é a faixa onde as estrelas permanecem o maior tempo de sua evolução, durante a transformação do hidrogênio em hélio. Elas ingressam sempre pela parte inferior da faixa. Estrelas de pequena massa na parte inferior, as de massa intermediária na parte central e as de grande massa na parte superior da sequência principal. 
O tempo de duração da queima do hidrogênio depende da massa inicial da estrela, o que significa que pode durar milhões de anos para estrela mais massivas ou até chegar a bilhões de anos para estrelas menos massivas, como o Sol. Entretanto, com a diminuição da quantidade do gás devido a queima, a temperatura e densidade precisam subir para manter a mesma taxa de fusão nuclear. O aumento da temperatura se dá de modo gradual e a estrela se expande levemente, o que resulta em um maior fluxo de energia para a superfície e no aumento da luminosidade. Com uma grande quantidade de hidrogênio transformado em hélio, o peso molecular médio muda, alterando a estrutura do astro. Inicialmente estas mudanças são graduais, mas posteriormente se dão de modo mais rápido. A relação massa-luminosidade, calculada por Arthur S. Eddington em 1924 [36], mostra que

$$
\frac{L_{*}}{L_{\odot}}=\left(\frac{M_{*}}{M_{\odot}}\right)^{\alpha},
$$

sendo $1<\alpha<6$ definido de acordo com a massa da estrela [37] e os símbolos $(\odot)$ e $(*)$ referem-se ao Sol e a estrela sob estudo, respectivamente. Estrelas da Sequência Principal geralmente recebem o valor $\alpha=3.5$. O tempo de vida $t$ de uma estrela que depende de sua energia (massa) e a taxa com que ela gasta essa energia (luminosidade). Então,

$$
\frac{t_{*}}{t_{\odot}}=\frac{\left(\frac{M_{*}}{M_{\odot}}\right)}{\left(\frac{L_{*}}{L_{\odot}}\right)}=\frac{\left(\frac{M_{*}}{M_{\odot}}\right)}{\left(\frac{M_{*}}{M_{\odot}}\right)^{3.5}}=\left(\frac{M_{*}}{M_{\odot}}\right)^{-2.5}
$$

conclui-se que quanto maior é a massa da estrela, menor é o tempo de vida dela.

Passados vários milhões de anos, às vezes até mesmo bilhões, a quantidade de hidrogênio diminui bastante, produzindo mudanças na estrutura da estrela. Uma das mudanças é a diminuição da pressão interna, o que faz com que a estrela comece a contrair-se. Esta ação libera energia gravitacional, que adicionada a da queima do hidrogênio, ainda existente, vence a força da gravidade e empurra as camadas mais externas para fora. Em suma, nesta fase o núcleo da estrela se contrai enquanto que as camadas externas se expandem. É o começo do fim.

A estrela sai da Sequência Principal para se tornar uma estrela gigante 
vermelha $^{5}$.

\subsection{3}

\section{Destinos das Estrelas}

Como mencionado acima, o modo de evolução dependerá muito da massa da estrela quando localizada na sequência principal do diagrama H-R. Embora os limites não sejam rígidos, podemos mostrar a evolução estelar da seguinte maneira:

\section{Pouquíssima Massa}

Estrelas com massas entre 0.08 e 0.8 massas solares são classificadas como estrelas de pouquíssima massa. O núcleo da estrela se contrai muito lentamente e a temperatura central não aumenta muito. O envoltório continua a se expandir e ela torna-se uma estrela supergigante. Como a expansão é contínua, em um dado momento a estrela não consegue mais manter gravitacionalmente o seu envoltório e este é ejetado no espaço. O destino final dela é tornar-se uma nebulosa planetária ${ }^{6}$.

\section{Pouca Massa}

Esta é a chamada região de massa solar, pois é onde está incluso a estrela do nosso sistema solar, o Sol. Ela vai de 0.8 até 3 massas solares. Sofrendo uma ação maior da gravidade, o núcleo da estrela consegue se contrair e sua temperatura se eleva a aproximadamente $10^{8} \mathrm{~K}$, quando inicia-se a queima do hélio em carbono através do processo triplo-alpha

$$
{ }^{4} \mathrm{He}+{ }^{4} \mathrm{He}+{ }^{4} \mathrm{He} \rightarrow{ }^{12} \mathrm{C}+\gamma \quad(7,5 \mathrm{MeV}) .
$$

Após consumir praticamente toda sua reserva de hélio, a estrela sofre o mesmo processo que passado na fase do hidrogênio: o núcleo se contrai e o envoltório se expande ainda mais. Temos uma supergigante. Como o núcleo não conseguirá contrair-se o suficiente para iniciar a queima do carbono, o seu destino é ejetar as camadas exteriores e transformar-se em nebulosa planetária.

\footnotetext{
${ }^{5}$ Esta denominação deve-se ao fato da estrela aumentar em até 200 vezes de tamanho e possuir temperatura superficial baixa, em torno de $3000 \mathrm{~K}$.

${ }^{6}$ Como a estrela expele as suas camadas mais externas, sua parte central e quente fica exposta. A forte radiação ultravioleta proveniente desta estrela residual faz o gás ejetado fluorescer.
} 


\section{Massa Intermediária}

São estrelas entre 3 e 8 massas solares. Neste intervalo o núcleo da estrela consegue atingir a temperatura de $10^{9} \mathrm{~K}$ e iniciar a queima do carbono através dos processos

$$
\begin{gathered}
{ }^{12} \mathrm{C}+{ }^{12} \mathrm{C} \rightarrow{ }^{24} \mathrm{Mg}, \\
{ }^{12} \mathrm{C}+{ }^{12} \mathrm{C} \rightarrow{ }^{20} \mathrm{Ne}+\alpha, \\
{ }^{12} \mathrm{C}+\alpha \rightarrow{ }^{16} \mathrm{O} .
\end{gathered}
$$

Embora não haja unanimidade, acredita-se que a estrela possa ter dois destinos:

1. A energia necessária para quebrar o núcleo de carbono sólido é tão grande que a estrela explodirá como uma supernova e não restará nada, a não ser o gás lançado no espaço; ou

2. A explosão não destruirá completamente a estrela e deixará uma estrela residual, muito pequena e densa, uma estrela de nêutrons.

\section{Muita Massa}

A partir de $\sim 8 M_{\odot}$ consideramos que uma estrela é massiva e, como a força gravitacional é bem maior para esta região, as estrelas passam por vários estágios de queima nuclear. Assim como as estrelas de menor massa, elas queimam hidrogênio, hélio e carbono; mas vão além devido a contração lenta e suave do núcleo. A temperatura consegue chegar a aproximadamente $2 \times 10^{9} \mathrm{~K}$, o que é suficiente para queimar os resíduos da queima do carbono: oxigênio e neônio. Os novos elementos da fusão anterior serão silício, enxofre e magnésio. Esta transformação se dá pelos seguintes processos:

$$
\begin{gathered}
{ }^{16} \mathrm{O}+{ }^{16} \mathrm{O} \rightarrow{ }^{28} \mathrm{Si}+\alpha \\
{ }^{16} \mathrm{O}+{ }^{16} \mathrm{O} \rightarrow{ }^{32} \mathrm{~S},
\end{gathered}
$$




$$
{ }^{20} \mathrm{Ne}+\alpha \rightarrow{ }^{24} \mathrm{Mg}
$$

Caso a estrela possua massa superior a 15 ou 20 massas solares, o seu núcleo consegue alcançar temperaturas de $3 \times 10^{9} \mathrm{~K}$, o que permite a queima nuclear chegar até a última escala, o Ferro, que é o elemento pesado mais estável. Nesta etapa, a estrela queima vários tipos de elementos, cada um em uma camada: a de hidrogênio, mais externa; e junto ao núcleo, o silício. Diz-se que a estrela possui estrutura interna semelhante a uma cebola [35]. Veja a Fig. 2.3.

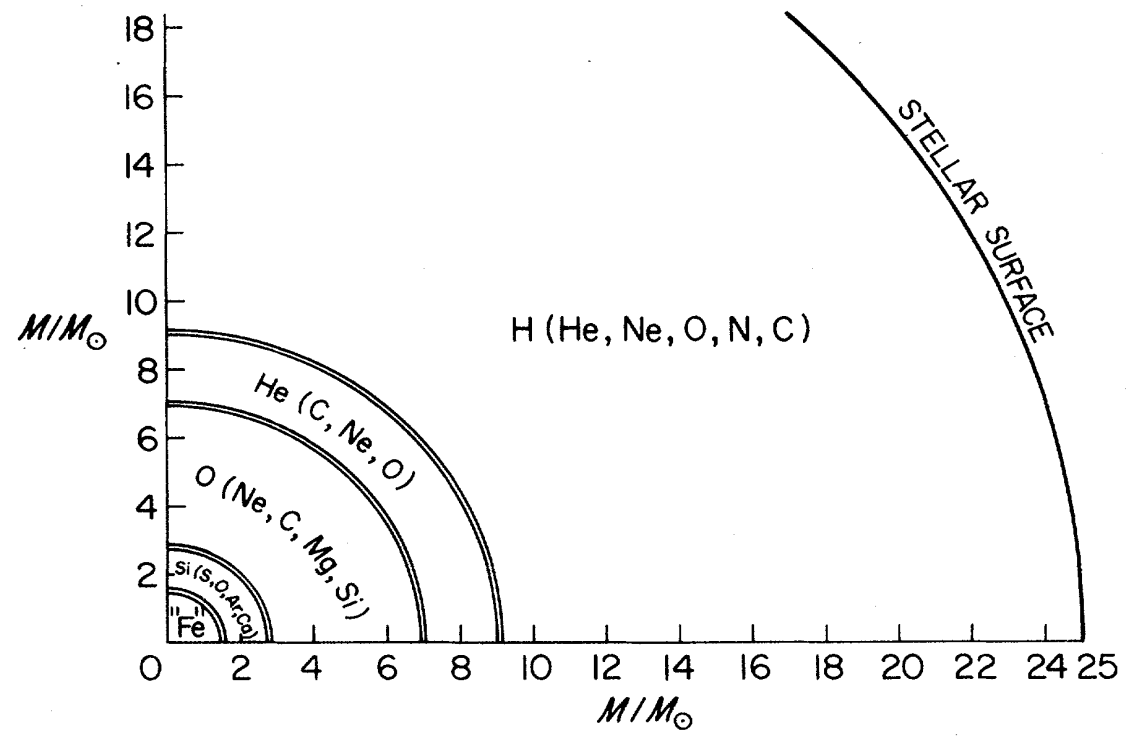

Figura 2.3: Camadas da queima nuclear de uma estrela muito massiva ao final de sua evolução. Figura de Mafalda Martins, ESO.

Vimos que estrelas com muita massa sofrem um processo violento ao final da queima de seu combustível nuclear. Estas são as explosões de supernovas. Como este é o evento que nos interessa neste trabalho, vamos nos aprofundar nesta parte e vê-la acontecer detalhadamente.

\section{3}

\section{Tipos de Supernovas}

As primeiras classificações de supernovas ocorreram no final da década de 30, por Zwicky e seu assistente, J.J. Johnson, que realizaram uma busca minuciosa no céu e encontraram aproximadamente 20 supernovas. Na mesma época, Minkowski mediu o espectro das supernovas descobertas. Pelo estudo das linhas espectrais, postulou-se que havia 2 tipos de supernovas, classificando-as 


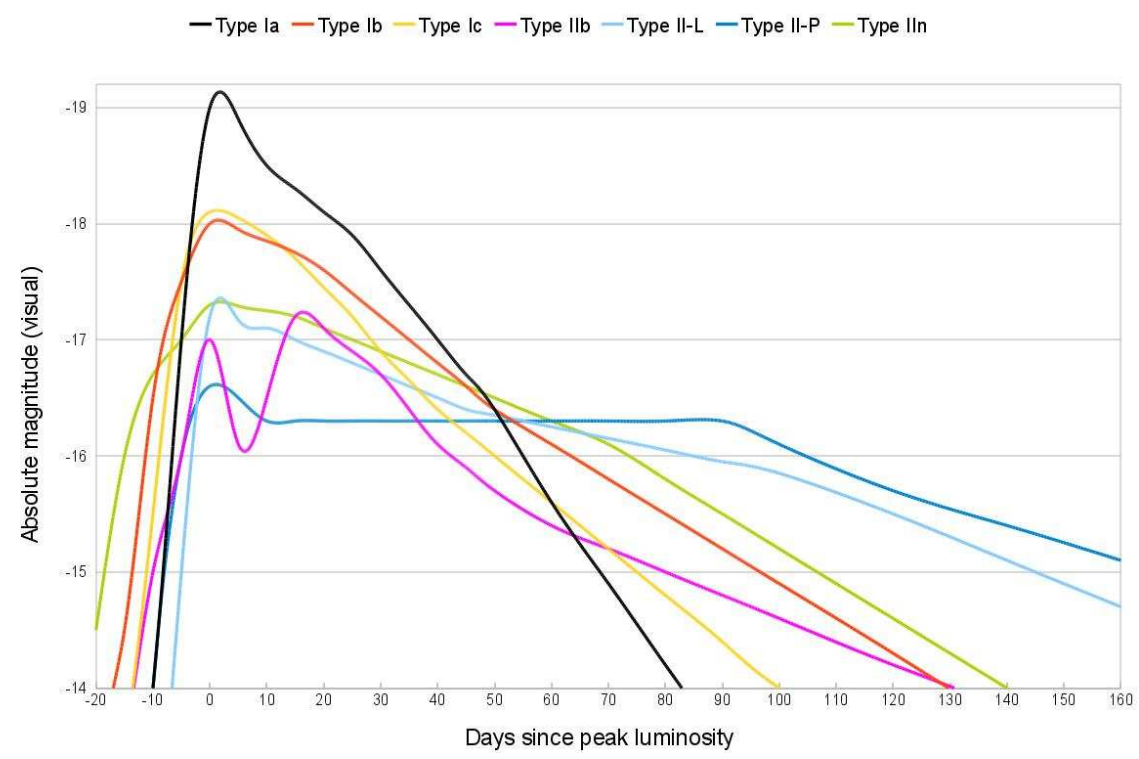

Figura 2.4: Curva de luz de SNe dos Tipos I e II.

como Tipo I e Tipo II. A principal característica é a ausência (presença) da linha espectral do hidrogênio para o tipo I (tipo II). Mas o fator mais importante é o processo de criação de supernovas, que pode ser termonuclear ou por colapso do núcleo.

Supernovas do Tipo I possuem curva de luz característica com um pico de luminosidade que dura em média 2 semanas e depois lenta e suavemente decresce de intensidade nos meses seguintes (vide Fig. 2.4). São divididas em tipos Ia, Ib e Ic.

Explosões do tipo Ia apresentam linha espectral de silício - formada da fusão de carbono com oxigênio - e são encontradas em todo tipo de galáxia: elíptica, espiral e irregular. Acredita-se que SN do tipo Ia ocorrem em regiões de estrelas muito velhas, e por isso pensa-se que são geradas por anãs-brancas ${ }^{7}$ com núcleo de carbono ou oxigênio que tem uma companheira próxima, formando um sistema binário. Esta companheira perde massa, através de inúmeros mecanismos, que é atraída gravitacionalmente pela anã-branca. Essa acreção de massa tem um limite, o limite de Chandrasekhar. Quando a anãbranca ultrapassar este limite, a pressão de degenerescência dos elétrons não

${ }^{7}$ Uma estrela de pouca massa ou intermediária $\left(\lesssim 8 M_{\odot}\right)$ transforma-se em gigante vermelha e, na queima do hélio ou carbono, ejeta suas camadas mais externas para o espaço, restando apenas o núcleo. Tudo isso - núcleo e gás lançado ao espaço - passa a ser chamado de nebulosa planetária. Após alguns milhares de anos, todo o gás lançado no espaço se dissipa e o núcleo da antiga estrela torna-se visível. Temos uma anã-branca, muito pequena e muito densa. 
mais suportará o puxão gravitacional e a estrela começa a colapsar, iniciando a fusão do carbono e oxigênio em metais mais pesados, que irá liberar uma quantidade enorme de energia e resultará na explosão termonuclear [30, 38]. Podemos calcular este valor máximo da seguinte maneira [39]: consideramos uma anã-branca com $N$ elétrons e de raio $R$ tal que o número de densidade eletrônica é $n \sim N / R^{3}$. E, de acordo com o Princípio de Exclusão de Pauli, o volume por elétron é $v \sim 1 / n$, com um tamanho $r \sim v^{1 / 3}$. Usando o Princípio de Incerteza de Heisenberg em unidades naturais temos que o momento do elétron é $p \sim r^{-1} \sim n^{1 / 3}$. Para estrelas com pouca massa, a pressão de degenerescência eletrônica consegue igualar-se com a gravidade e os elétrons não são relativísticos. Os elétrons de estrelas massivas possuem regime relativístico devido a alta densidade alcançada no núcleo durante a contração do astro. Neste caso podemos desprezar a massa do elétron e escrever a energia devido a pressão deste gás de Fermi como

$$
E_{p} \simeq p \sim \frac{N^{1 / 3}}{R}
$$

Cada elétron é associado com uma massa $m \equiv m_{N} / Y_{e}$, onde $m_{N}$ é a massa de um nucleon e $Y_{e}$ é a fração eletrônica ${ }^{8}$. Assim, a energia gravitacional por elétron é

$$
E_{G} \sim-\frac{G M m}{R} \sim-\frac{G m_{N}^{2} N Y_{e}^{-2}}{R}
$$

onde $G$ é a constante gravitacional ${ }^{9}$ e a massa total é $M \simeq m N \simeq m_{N} N / Y_{e}$. Podemos observar que o equlilíbrio é alcançado quando a energia total é mínima

$$
E=E_{P}+E_{G} \sim \frac{N^{1 / 3}}{R}-\frac{G m_{N}^{2} N Y_{e}^{-2}}{R}
$$

Importante observar que ambas energias variam em $1 / R$. Para não haver

${ }^{8}$ Expressada matematicamente por $Y_{e}=\frac{N_{p}}{N_{p}+N_{n}}$, sendo $N_{p}$ e $N_{n}$ os números de prótons e nêutrons, respectivamente.

${ }^{9}$ A Constante da Gravitação Universal expressa a atração gravitacional que é produzida entre dois corpos de um quilograma cada, separados por uma distância de um metro, com valor atual dado por

$$
G=6.6743 \times 10^{-11} \mathrm{~m}^{3} \mathrm{~kg}^{-1} \mathrm{~s}^{-2} .
$$


colapso é necessário que a energia seja não negativa. Então podemos determinar o número máximo de elétrons fazendo $E=0$ em (2.12):

$$
N_{\max } \sim\left(G m_{N}^{2} Y_{e}^{-2}\right)^{-3 / 2} \simeq 2 \times 10^{57} Y_{e}^{3},
$$

e a massa para esta quantidade é a massa de Chandrasekhar

$$
M_{\max } \sim N_{\max } m_{N} Y_{e}^{-1}
$$

Utilizando dados de cálculos mais precisos [39, 40], a eq. (2.14) produz

$$
M_{\text {max }} \simeq 5.83 Y_{e}^{2} M_{\odot} \simeq 1.46 M_{\odot}
$$

onde utilizamos o valor típico (0.5) para $Y_{e}$. Quando a energia da eq. (2.12) torna-se negativa o colapso começa, engatilhando a fusão de carbono e oxigênio em elementos mais pesados, que libera uma quantidade enorme de energia, da ordem de $10^{51}$ erg causando a explosão termonuclear da estrela. Levando em consideração a temperatura da anã-branca $(T \leq 1 \mathrm{MeV})$, sua densidade $\left(\rho_{c} \leq 10^{10}\right.$ g.cm $\left.{ }^{-3}\right)$ e o caminho livre médio do neutrino espalhado pelo núcleo do ${ }^{56} \mathrm{Ni}$ que é da ordem de $10^{8} \mathrm{~cm}$ (maior que o raio da anã-branca - $O\left(10^{7}\right.$ ) $\mathrm{cm})$, vemos que o neutrino escapa livremente e sem ser termalizado. Com isso, neutrinos levam apenas uma pequena fração da energia (menor que $O\left(10^{49}\right)$ erg) e não desempenham um papel importante nas explosões de supernovas termonucleares [30, 41].

As supernovas dos tipos Ib e Ic não apresentam a linha espectral do hidrogênio, o que as classifica como pertencente ao Tipo I, mas também não possuem a linha de silício. Elas são observadas em regiões onde há estrelas jovens, o que indica que são estrelas de vida curta, porém mais massivas. Antes de explodirem como supernovas, essas estrelas perderam a sua camada de hidrogênio. Então, qual é a diferença entre os tipos Ib e Ic? O que os distingue é que uma supernova do Tipo Ib apresenta riqueza em hélio enquanto que a do Tipo Ic mostra ausência deste elemento, pois também perdeu sua concha de hélio enquanto ainda era uma estrela [42].

Estrelas que não consumiram ou perderam suas camadas de hidrogênio mais externas, acabam sua vida na forma de supernovas do Tipo II. Esses eventos são observados comumente nos braços de galáxias espirais, mas ocasionalmente registra-se também em galáxias irregulares [43]. Seus progenitores 


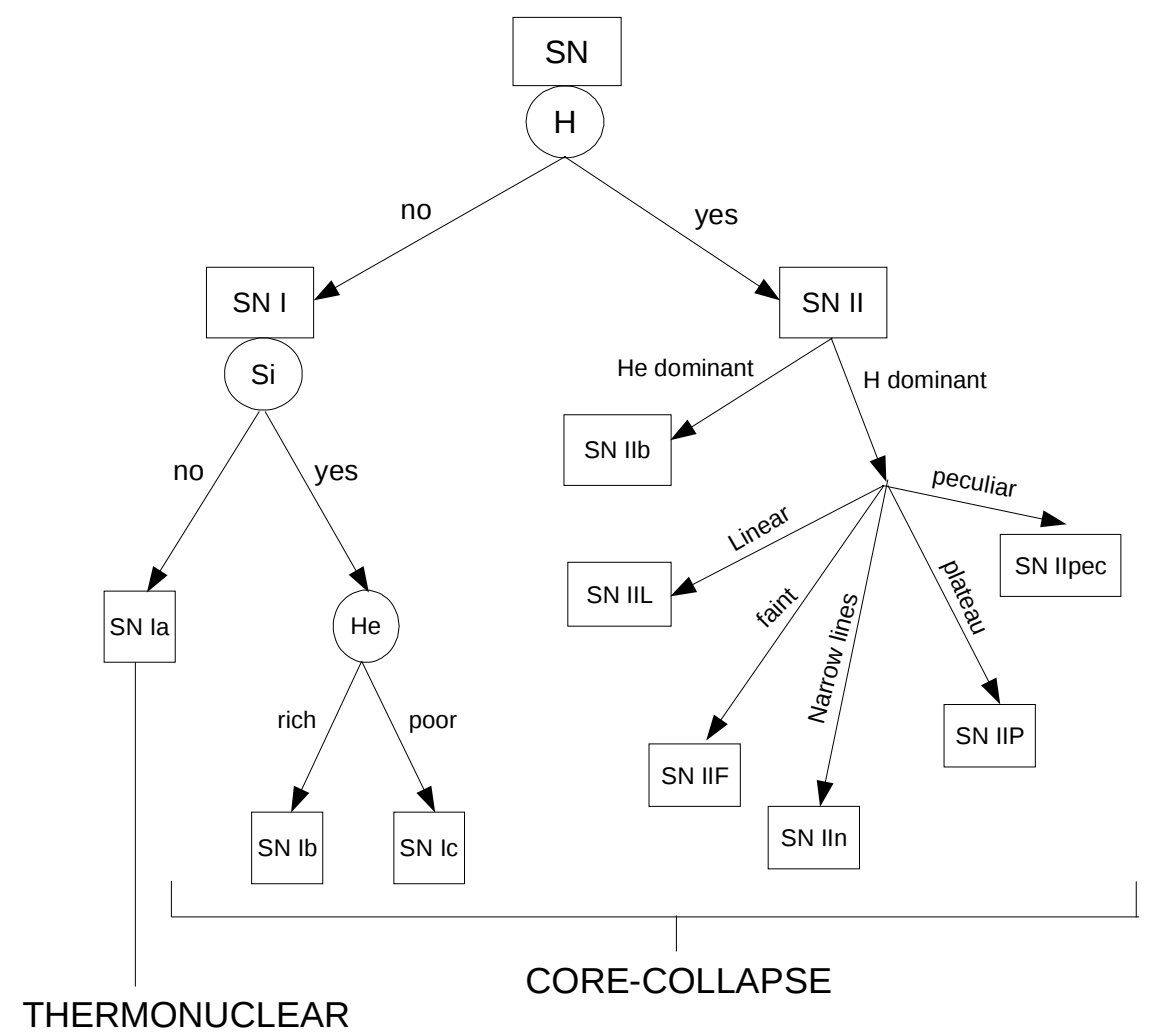

Figura 2.5: Quadro de classificação de Supernovas. Reproduzido da Ref. [38].

eram massivos $\left(>8 M_{\odot}\right)$ e por isso tiveram vida curta (ver eq.(2.2) ). Outra diferença em relação à do Tipo I é que o decaimento da luminosidade é mais brusco no primeiro mês após o seu máximo e depois a queda é mais lenta, formando um platô no seu espectro (ver Fig. 2.4). Como os efeitos visuais deste tipo de acontecimento variam muito de um caso para outro, há muitos subtipos de SN do Tipo $\mathrm{II}^{10}$, como podemos observar na Fig. 2.4.

Do ponto de vista da física de neutrinos, as supernovas tipos Ib, Ic e II são mais interessantes que as do tipo Ia pois produzem uma quantidade enorme de neutrinos. Essa diferença deve-se ao fato de que o processo de criação da supernova do tipo Ia é através de explosão termonuclear, enquanto que os demais são por colapso do núcleo, conforme pode ser visto no esquema da Fig. 2.5, reproduzido da Ref. [38].

O objeto central quando do colapso da estrela é um corpo compacto, muito quente e com alta taxa de produção de neutrinos de todas as gerações. São os neutrinos que levam aproximadamente $99 \%$ da energia de ligação gravitacional da estrela ( $\left.3 \times 10^{53} \mathrm{erg}\right)$, enquanto que somente $1 \%$ é perdido

${ }^{10}$ para um aprofundamento neste ponto recomendamos a leitura das Ref. [44, 45]. 


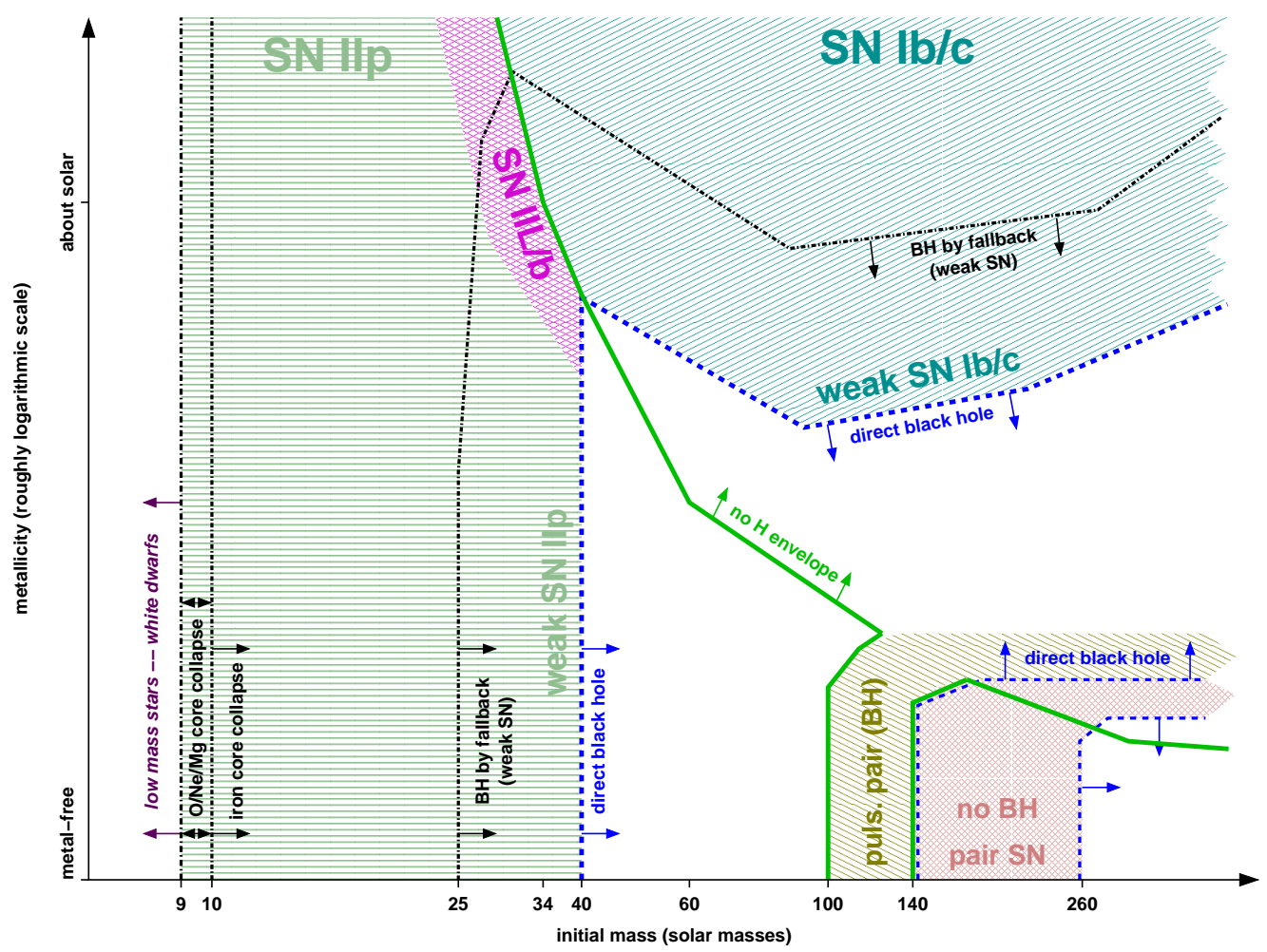

Figura 2.6: Tipos de SN de estrelas massivas em função da massa e metalicidade iniciais.

através de energia cinética e menos ainda $(0,01 \%)$ na forma de radiação.

Após a explosão de supernova (SN), restará da estrela original uma estrela de nêutrons ou teremos a formação de um buraco negro. Este resultado dependerá da massa da progenitora ao ingressar na sequência principal, milhões de anos antes. A Fig. 2.6 nos mostra um gráfico dos possíveis destinos da estrela em função de sua massa inicial e metalicidade (elementos pesados) [46].

\section{4}

\section{Etapas da Explosão de uma Supernova (SN)}

\subsection{1}

\section{Pré-Colapso}

Antes de começar o colapso da região central da estrela, há uma queda brusca na função densidade entre o núcleo (manto) e as camadas vizinhas, conforme podemos ver na Fig. 2.7 [47]. Isto faz com que a escala de tempo dinâmica do núcleo seja muito menor que nas zonas circundantes. Por isto, o que acontece nestas regiões de elementos mais leves não influencia na dinâmica 
nuclear e, daqui em diante, acompanharemos apenas o que acontece no núcleo estelar.

\section{4 .2}

\section{Início do Colapso}

Basicamente, são três os processos que podem desencadear o colapso da estrela. Em geral, podemos dizer que o núcleo de uma estrela massiva ( $\mathrm{O}, \mathrm{Ne}, \mathrm{Mg}$ ou $\mathrm{Fe}$ ) aumenta com as cinzas da queima nuclear nas camadas externas vizinhas a ele, e faz com que sua massa se aproxime do limite de Chandrasekhar. O raio nuclear é de poucos milhares de quilômetros $\left(\sim 10^{4}\right.$ $\mathrm{km})$, com uma densidade em torno de $10^{10} \mathrm{~g} \cdot \mathrm{cm}^{-3}$ e temperatura de $10^{10} \mathrm{~K}$ $(1 \mathrm{MeV})$. A gravidade é contrabalançada pela pressão dos elétrons degenerados, mas com o aumento da massa o núcleo se contrai e a temperatura aumenta.

Assim, para estrelas de menor massa $\left(8 M_{\odot}<M_{*}<10 M_{\odot}\right)$ [47, 48], cujos núcleos são compostos de oxigênio, neônio e magnésio ( $\mathrm{O}, \mathrm{Ne}, \mathrm{Mg}$ ), resultado da queima do carbono, a degenerescência eletrônica ocorre antes de haver condições para iniciar a queima do Ne. A captura eletrônica pelos elementos do núcleo se dá, por exemplo,

$$
{ }^{24} \mathrm{Mg}+e^{-} \rightarrow{ }^{24} \mathrm{Na}+\nu_{e},
$$

ou de maneira geral

$$
e^{-}+N(Z, A) \rightarrow N(Z-1, A)+\nu_{e}
$$

e também por prótons livres

$$
e^{-}+p \rightarrow n+\nu_{e}
$$

devido a alta energia de Fermi dos elétrons [30]

$$
\mu_{e} \sim 11.1\left(\frac{\rho Y_{e}}{10^{10} \mathrm{~g} / \mathrm{cm}^{3}}\right)^{1 / 3} \mathrm{MeV} .
$$

A diminuição na quantidade de elétrons faz com a pressão exercida por eles diminua e o núcleo começa a colapsar, aumentando a densidade no núcleo da estrela, que por sua vez aumenta a energia de Fermi, gerando uma maior taxa 

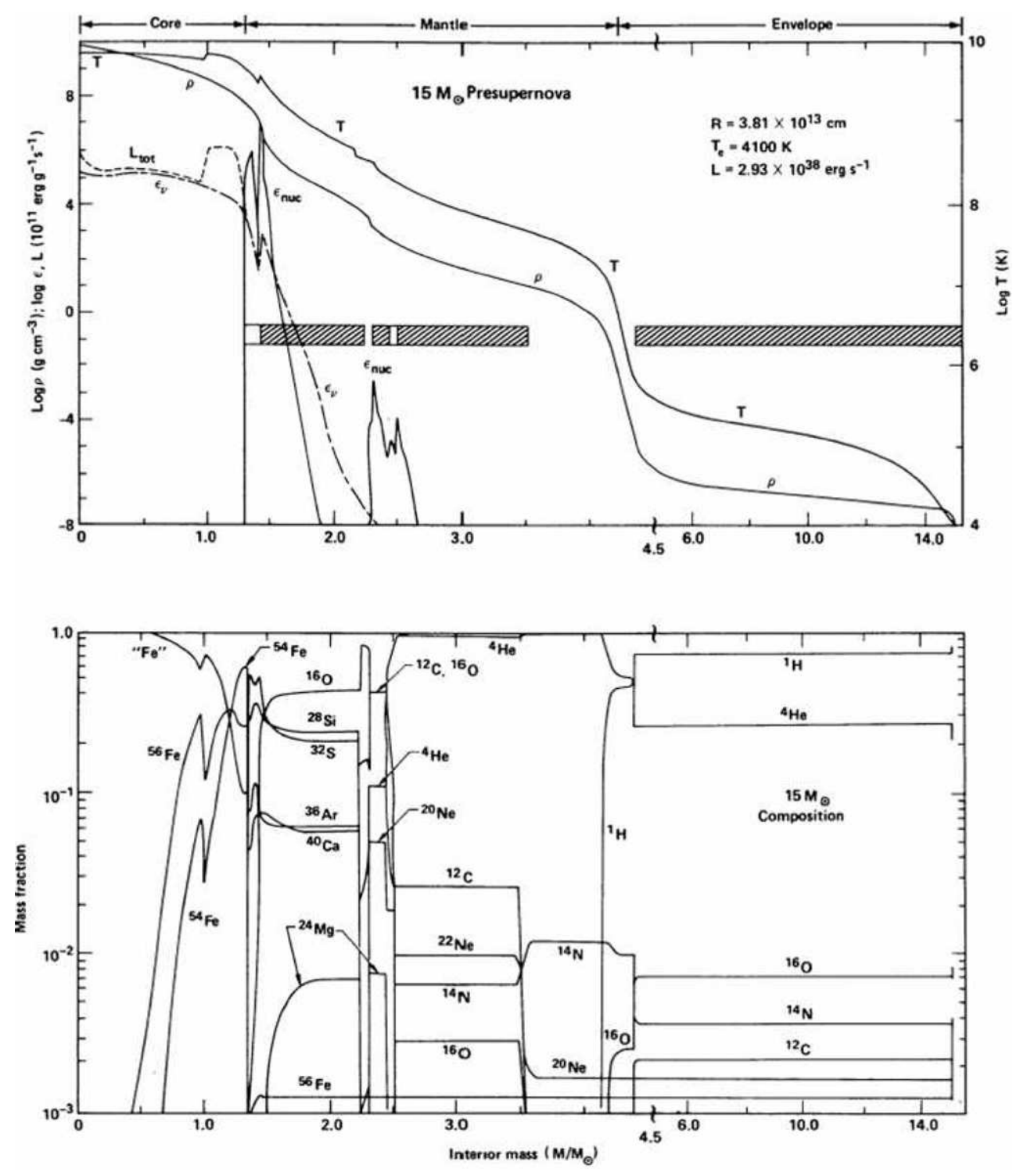

Figura 2.7: Estrutura de uma estrela de $15 \mathrm{M}_{\odot}$ antes de colapsar. Superior: dentro do núcleo a emissão de neutrinos $\left(\epsilon_{\nu}\right)$, gerados via captura eletrônica, responde por quase a totalidade da perda de energia da estrela $\left(L_{t o t}\right)$. A energia gerada pela queima dos elementos pode ser observada pelos picos na taxa de geração de energia nuclear $\left(\epsilon_{n u c}\right)$. Inferior: a distribuição dos elementos químicos dentro de uma estrela de $15 M_{\odot}$. No núcleo, "Fe" inclui todos os isótopos entre $48 \lesssim A \lesssim 65$. Figura retirada da Ref. [47]. 
de captura eletrônica, acelerando o colapso. Este tipo de SN contribui com uma taxa de $\sim 20 \%$ do total de SN $[49,50]$.

Os neutrinos eletrônicos produzidos pelas eqs. (2.17) e (2.18) conseguem sair rapidamente do núcleo, levando consigo energia e número leptônico. Isso é possível porque seu livre caminho médio é maior que o raio nuclear. Como dito anteriormente, a luminosidade de uma supernova chega na ordem de $10^{53} \mathrm{erg} . \mathrm{s}^{-1}$, mas nesta fase o brilho é de apenas $1 \%$ deste valor, pois o período de duração da captura eletrônica é muito curto, menor que 10 ms [31].

Estrelas com mais massa conseguem queimar o $\mathrm{Ne}$, formando um núcleo de Fe em seus centros. O ferro é o elemento mais estável da natureza (com maior energia de ligação) e por isso não queimará, mesmo com o aumento da temperatura; e a estrela ficará sem combustível. Mas o aumento da temperatura causa o processo de fotodissociação do núcleo de ferro, liberando partículas $\alpha$ e aumentando o número de nucleons livres,

$$
\gamma+{ }^{56} \mathrm{Fe} \rightarrow 13 \alpha+4 n
$$

Essa reação absorve aproximadamente $124 \mathrm{MeV}$ de energia e reduz a energia cinética e a pressão dos elétrons. Com a pressão menor, diminui o valor da massa de Chandrasekhar até esta tornar-se menor que a massa do núcleo da estrela. Quando este limite é alcançado, a pressão dos elétrons degenerados não consegue mais contrabalançar o puxão gravitacional e começa o colapso. Com este acontecimento, a temperatura na parte central da estrela aumenta e gera mais fotodissociação, que acelera o colapso.

Estrelas entre $100 M_{\odot}<M_{*}<140 M_{\odot}$ e acima de $M_{*}>260 M_{\odot}$ sofrem instabilidade gravitacional devido a formação de pares $e^{-} e^{+}$de fótons de alta energia, que transforma energia térmica em energia de massa de repouso. Isto acaba por reduzir o índice de adiabaticidade abaixo do valor (4/3) que mantém a estabilidade hidrodinâmica da estrela, iniciando o colapso da mesma e resultando no surgimento de um buraco negro. Finalmente, estrelas cujas massas caem neste intervalo, sofrem uma violenta explosão termonuclear forte o suficiente para destruir totalmente a estrela, sem deixar remanescente e lançando ao espaço $>50 M_{\odot}$ de ${ }^{56} \mathrm{Ni}[46,51]$. 


\section{4 .3}

\section{Aprisionamento de Neutrinos}

A descoberta da interação fraca de corrente neutra (NC, em inglês) possibilitou que Freedman [52], Sato [53, 54] e Mazurek [55] postulassem o aprisionamento de neutrinos. Este tipo de interação aumenta consideravelmente o espalhamento elástico dos neutrinos pelos nucleons (predominantemente os nêutrons, mas também os prótons - em escala muito menor).

O comprimento de onda do neutrino é

$$
\lambda \sim \frac{\hbar c}{E_{\nu}}=20\left[\frac{E_{\nu}}{10 \mathrm{MeV}}\right]^{-1} \mathrm{fm}
$$

cujo valor é maior que o tamanho do núcleo atômico do ferro

$$
R_{\mathrm{Fe}} \sim 5(A / 56)^{1 / 3} \mathrm{fm}
$$

Então, eles são espalhados coerentemente pelos núcleos dos átomos de Fe com a consequência de termos o processo de decaimento beta inverso ( eq.(2.18)). Sabemos que o caminho livre médio do neutrino pode ser calculado por

$$
\lambda_{\nu}=\frac{1}{\sigma_{A} n_{A}}
$$

onde $n_{A}=\rho /\left(A m_{u}\right)$ é a densidade de núcleos atômicos $\left(m_{u}\right.$ é massa em unidades de massa atômica) e $\sigma_{A}$ é a seção de choque de espalhamento coerente que podemos escrever [56]

$$
\sigma_{A}=\frac{1}{16} \sigma_{0}\left(\frac{E_{\nu}}{m_{e} c^{2}}\right)^{2} A^{2}\left[1-\frac{Z}{A}+\left(4 \sin ^{2} \theta_{w}-1\right) \frac{Z}{A}\right]^{2}
$$

sendo que

$$
\sigma_{0}=\frac{4 G_{F}\left(m_{e} c^{2}\right)^{2}}{\pi(\hbar c)^{4}}=1.705 \times 10^{-44} \mathrm{~cm}^{2}
$$

é a seção de choque de referência para interação fraca e $G_{F}$ e $\theta_{w}$ são as constantes de acoplamento de Fermi e o ângulo de Weinberg ${ }^{11}$, respectivamente. Como os elétrons envolvidos no processo de captura passarão toda sua energia

${ }^{11}$ Atualmente os melhores valores obtidos experimentalmente são [57]:

$$
G_{F}=1.166 \times 10^{-5} \mathrm{GeV}^{-2} \text { e } \sin ^{2} \theta_{W}=0.231 .
$$


para o neutrino [31], podemos estimar a energia média do neutrino eletrônico no núcleo de ferro como

$$
E_{\nu}=\frac{\int_{0}^{\mu_{e}} E E^{2} E^{2} d E}{\int_{0}^{\mu_{e}} E^{2} E^{2} d E}=\frac{5}{6} \mu_{e}
$$

substituindo (2.19) em (2.26) e arrumando as frações temos

$$
E_{\nu}=10.3\left(\frac{\rho}{3 \times 10^{10} \mathrm{~g} \mathrm{~cm}^{-3}}\right)^{1 / 3}\left(\frac{Y_{e}}{26 / 56}\right)^{1 / 3} \mathrm{MeV}
$$

Introduzindo as equações (2.24) e (2.27) na eq. (2.23) o livre caminho médio será

$$
\lambda_{\nu}=85\left(\frac{\rho}{3 \times 10^{10} \mathrm{~g} \mathrm{~cm}^{-3}}\right)^{-5 / 3}\left(\frac{A}{56}\right)^{-1}\left(\frac{Y_{e}}{26 / 56}\right)^{-2 / 3} \mathrm{~km} .
$$

Considerando o núcleo esférico, sem rotações, podemos estimar o seu raio através das equações de volume $\left(V=\frac{4 \pi R^{3}}{3}\right)$ e densidade $\left(\rho=\frac{M}{V}\right)$

$$
\begin{aligned}
R_{\text {núcleo }} & \approx\left(\frac{3 M_{\text {núcleo }}}{4 \pi \rho}\right)^{1 / 3} \\
& \approx 2.7 \times 10^{2}\left(\frac{\rho}{3 \times 10^{10} \mathrm{~g} \mathrm{~cm}^{-3}}\right)^{-1 / 3}\left(\frac{Y_{e}}{26 / 56}\right)^{2 / 3} \mathrm{~km},
\end{aligned}
$$

e comparando as equações (2.28) e (2.29) tiramos dois resultados:

1. O livre caminho médio do neutrino torna-se menor que o tamanho do núcleo de ferro, e

2. A dependência do livre caminho médio com a densidade é $\propto \rho^{-5 / 3}$ enquanto que o raio possui uma dependência $\propto \rho^{-1 / 3}$. Então, com o aumento da densidade, os neutrinos não conseguirão escapar livremente do núcleo estelar.

Tudo isto indica que há a formação de uma região superficial que determina se os neutrinos escaparão livres (posição radial além desta superfície) ou ficarão presos nela (aquém desta superfície). Tal região é conhecida como neutrinosfera. Para encontrarmos a posição radial da neutrinosfera devemos 
calcular a sua superfície limítrofe quando o valor da profundidade ótica ${ }^{12}$ se aproxima da unidade. O valor comumente usado é $2 / 3[31,58]$ pois é levado em consideração que o neutrino não sairá radialmente e sem interação, mas sim com algum ângulo. Escrevemos a profundidade ótica como

$$
\tau\left(r, E_{\nu}\right)=\int_{r}^{\infty} \frac{d r}{\lambda_{\nu}}
$$

e substituimos as eqs. (2.27) e (2.28) em (2.30) com $A=56$ como o valor típico do ferro para obtermos

$$
\tau\left(r, E_{\nu}\right)=\frac{\left(8.5 \times 10^{6}\right)^{-1}}{\left(3 \times 10^{10}\right)}\left(\frac{E_{\nu}}{10}\right)^{2} \int_{r}^{\infty} \rho(r) d r
$$

e podemos aproximar a distribuição de densidade como [31]

$$
\rho(r)=H r^{-3}
$$

onde $H=3 \times 10^{31} \mathrm{~g}$ é uma constante durante a fase de colapso. Então, a integral fica

$$
\begin{aligned}
\int_{r}^{\infty} \rho(r) d r & =\int_{r}^{\infty} H r^{-3} d r \\
& =\frac{H r^{-2}}{2}
\end{aligned}
$$

e utilizamos novamente a eq. (2.32) para fazermos $r=(H / \rho)^{1 / 3}$ e substituir em (2.33). Assim, a nova forma da eq. (2.31) será

$$
\tau\left(r, E_{\nu}\right)=\frac{1}{8.5 \times 10^{6}} \frac{1}{\left(3 \times 10^{10}\right)}\left(\frac{E_{\nu}}{10 \mathrm{MeV}}\right)^{2} \frac{H^{1 / 3}}{2} \rho^{2 / 3} .
$$

Substituindo $H$ pelo seu valor, juntamente com alguma aritmética obtemos a expressão final para a profundidade ótica como

$$
\tau\left(r, E_{\nu}\right) \approx 6.1\left(\frac{E_{\nu}}{10 \mathrm{MeV}}\right)^{2}\left(\frac{\rho}{10^{12} \mathrm{~g} \mathrm{~cm}^{-3}}\right)^{2 / 3}
$$

Pela eq. (2.35) e o valor usual da profundidade ótica $(\tau=2 / 3)$ podemos ${ }^{12}$ Profundidade ótica informa quão opaco um meio é para a radiação que passa através dele. 
obter a densidade da neutrinosfera

$$
\rho\left(R_{\nu}\left(E_{\nu}\right)\right)=3.6 \times 10^{10}\left(\frac{E_{\nu}}{10 \mathrm{MeV}}\right)^{-3} \mathrm{~g} \cdot \mathrm{cm}^{-3}
$$

e aplicando a eq. (2.32) em (2.36) encontramos a posição radial da neutrinosfera

$$
R_{\nu} \simeq 1.0 \times 10^{2}\left(\frac{E_{\nu}}{10 \mathrm{MeV}}\right) \mathrm{km}
$$

Pelas equações acima vemos que a posição da neutrinosfera depende da energia do neutrino. Então, nota-se que este valor varia de sabor para sabor. Da teoria de caminho aleatório podemos encontrar o tempo de difusão dos neutrinos para fora do núcleo estelar.

$$
t_{\text {dif }}=\frac{3 R_{\text {núcleo }}^{2}}{\lambda_{\nu} c} \simeq 3 \times 10^{-1}\left(\frac{\rho}{10^{12} \mathrm{~g} \mathrm{~cm}^{-3}}\right) \mathrm{s} .
$$

Depois, temos o tempo de colapso do núcleo, isto é, o tempo dinâmico

$$
t_{\mathrm{din}}=\frac{1}{\sqrt{G \rho}} \simeq 3.9 \times 10^{-3}\left(\frac{\rho}{10^{12} \mathrm{~g} \mathrm{~cm}^{-3}}\right)^{-1 / 2} \mathrm{~s}
$$

onde $G$ é a constante gravitacional.

Comparando as eqs. (2.38) e (2.39) vemos que quando a densidade é $\sim 10^{12}$ g.cm ${ }^{-3}$ os neutrinos não conseguem escapar livremente do centro da estrela porque o tempo de difusão é maior que o tempo do colapso, ou seja, eles estão presos. Este evento indica que a fração leptônica ${ }^{13}\left(Y_{l}=Y_{e}+Y_{\nu}\right)$ é mantida praticamente constante a partir deste momento. Uma vez presos, os neutrinos tornam-se degenerados, igual aos elétrons (Princípio de Pauli), e isto faz com que seja suprimido o processo de neutronização (transformação de prótons em nêutrons) via captura eletrônica. Este equilíbrio é chamado de equilíbrio $\boldsymbol{\beta}$ e, depois de alcançado, a entropia (matéria + neutrinos) é conservada no centro da estrela e o colapso continua de maneira adiabática.

${ }^{13} Y_{\nu}$ é a fração de neutrinos eletrônicos por barion, cuja expressão matemática é

$$
Y_{\nu}=n_{\nu_{e}}-n_{\bar{\nu}_{e}} .
$$




\section{4 .4}

\section{Colapso Homólogo}

O colapso do núcleo da estrela pode ser dividido em duas partes distintas: a primeira é a parte interna do núcleo que colapsa homologamente, isto é, velocidade subsônica e proporcional ao raio da massa em queda $(v \propto r)$; a segunda parte é a externa do núcleo, que cai praticamente em quedalivre com velocidade supersônica. Essa diferença de velocidades pode ser observada na Fig. 2.8 [59]. Goldreich e Weber [60] e Yahil e Lattimer [61] predisseram o colapso homólogo e mostraram que a pressão desaparece à massa de Chandrasekhar, isto é, somente a região interna do núcleo (dentro do limite de Chandrasekhar) colapsa homologamente. O tempo dinâmico da parte nuclear mais externa é maior que a parte mais central, fazendo com que a periferia do núcleo seja deixada para trás durante o colapso e por isso, podemos trabalhar como queda livre. É importante observar que o limite de Chandrasekhar depende de $Y_{e}^{2}$ (eq. 2.15), sendo que alguns trabalhos [62, 63] mostraram que o valor da fração eletrônica situa-se entre 0.35 e 0.38 ; isto nos indica que a massa que colapsa homologamente possui o valor entre 0.7 e 0.8 massas solares, estando de acordo com resultados de simulação [64].

\subsection{5}

Bounce Nuclear, Formação da Onda de Choque e Explosão de SN

O colapso do núcleo interno continua além do limite de densidade nuclear $\left(\approx 10^{14}\right.$ g.cm $\left.{ }^{-3}\right)$, mas a partir deste valor a pressão de repulsão dos nucleons degenerados diminui o colapso até que este pare. Como o índice de adiabaticidade $(\gamma)$ é maior que o valor crítico (4/3), há uma configuração hidrostática estável para o núcleo interno e, quando este contrai além desta configuração, o núcleo ricochetea, semelhante a uma mola comprimida quando uma de suas extremidades é solta. Neste instante a densidade atinge o seu máximo, de 3 a 4 vezes a densidade nuclear, e a este processo de "ricochetear" do núcleo interno damos o nome de "bounce". A região que agora encontra-se em equilíbrio forma uma proto estrela de nêutron com um raio em torno dos $10 \mathrm{~km}$, mas o movimento do bounce do núcleo interno, devido a inércia, gera uma onda de pressão que cresce e transforma-se em uma onda de choque na periferia do núcleo interno com velocidade da ordem de $100 \mathrm{~km} \mathrm{~ms}^{-1}$ [38]. Importante salientar que o núcleo interno não sofre com a onda de choque ou 


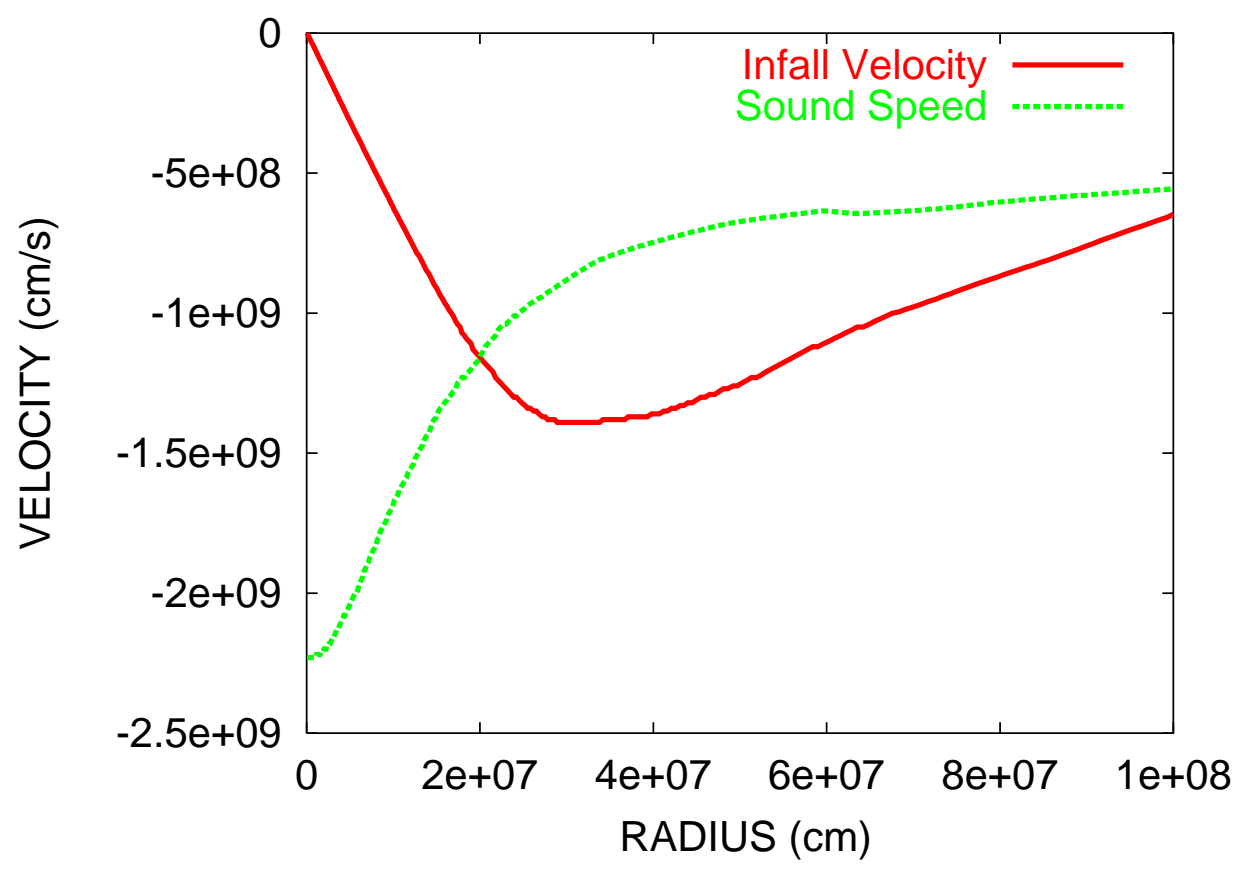

Figura 2.8: Velocidade de queda e velocidade do som em função do raio com densidade central de $10^{12} \mathrm{~g} \mathrm{~cm}^{-3}$ e massa do progenitor em $15 M_{\odot}$. O ponto onde as velocidades se encontram marca o limite entre os dois tipos de colapso que ocorrem no centro da estrela. Este ponto é chamado de ponto sônico.

com a onda de pressão, ele permanece praticamente da mesma forma que se encontrava quando do colapso homólogo.

Quando a onda de choque encontra a região do núcleo externo (região fora do limite de Chandrasekhar), que ainda cai com velocidade supersônica, ocorre uma desaceleração abrupta da matéria em queda e dissipação de energia da onda por fotodissociação dos núcleos atômicos, que estavam em queda livre, em nucleons livres. Prótons livres possuem uma alta taxa de captura eletrônica (eq. 2.17), isso cria uma quantidade enorme de neutrinos eletrônicos. Enquanto a onda não chega na região da neutrinosfera, os neutrinos eletrônicos não conseguem escapar e ficam presos logo atrás da onda de choque. A partir do momento que a neutrinosfera é alcançada, os neutrinos do elétron desacoplam da matéria e propagam-se livremente. Esta liberdade acontece subitamente e sua duração é menor que 20 ms. Chamamos este momento de explosão de neutronização ou explosão rápida do neutrino eletrônico ou ainda de pulso de fuga ${ }^{14}$. Devido ao curto tempo de duração do evento, a energia

${ }^{14} \mathrm{O}$ sinal deste aumento significativo de neutrinos eletrônicos é o que se espera identificar em detectores terrestres, como o Super-Kamiokande (SK), quando do nascimento de uma supernova galáctica. 
emitida é somente da ordem de $10^{51}$ erg [58].

A região onde a onda de choque passou encontra-se quente e não há uma grande quantidade de elétrons degenerados, permitindo que pósitrons relativísticos sejam criados e, a partir destes, anti-neutrinos eletrônicos $\left(\bar{\nu}_{e}\right)$ pela reação

$$
e^{+}+n \rightarrow p+\bar{\nu}_{e} .
$$

Também há a criação de todos os sabores de neutrinos (eletrônicos, muônicos e tauônicos) produzidos pela aniquilação de pares elétron-pósitron

$$
e^{-}+e^{+} \rightarrow \nu_{e, \mu, \tau}+\bar{\nu}_{e, \mu, \tau},
$$

por bremsstrahlung nucleon-nucleon

$$
N+N \rightarrow N+N+\nu_{e, \mu, \tau}+\bar{\nu}_{e, \mu, \tau},
$$

ou bremsstrahlung elétron-nucleon

$$
e^{ \pm}+N \rightarrow e^{ \pm}+N+\nu_{e, \mu, \tau}+\bar{\nu}_{e, \mu, \tau},
$$

assim como por decaimento de plasma

$$
\gamma \rightarrow \nu_{e, \mu, \tau}+\bar{\nu}_{e, \mu, \tau},
$$

e fotoaniquilação

$$
\gamma+e^{ \pm} \rightarrow e^{ \pm}+\nu_{e, \mu, \tau}+\bar{\nu}_{e, \mu, \tau},
$$

entre outros com menor porcentagem de participação.

Num raio de aproximadamente $200 \mathrm{~km}$ a onda de choque, gerada pela compressão do núcleo interno e que perdeu energia pelas dissociações nos átomos de ferro, corre o risco de parar o seu movimento e "morrer". Para que haja a explosão de supernova sem esta "morte" da onda de choque, são necessárias combinações especiais [65, 66] dos parâmetros físicos, mas de um modo geral podemos dizer que, se a estrela à época da sequência principal possuia até 10 massas solares, a onda de choque conseguirá sair do manto e expelir as camadas da estrela, gerando a explosão de SN. Todo este processo 
ocorre na ordem de 100 ms após o "bounce" do núcleo e recebe o nome de explosão rápida de SN.

Acima deste valor de massa, a onda de choque para aproximadamente após os $100 \mathrm{~ms}$ e a matéria do núcleo externo continua a cair e a sofrer o processo de fotodissociação dos átomos em nucleons livres que posteriormente geram neutrinos. Se $10 \%$ da energia destes neutrinos for transferida para a matéria próxima do local onde a onda de choque estagnou nos 300 ms seguintes, é possível que ela seja restaurada, possibilitando a explosão de SN [67]. Salientamos que a grande maioria desta energia proviria dos neutrinos eletrônicos, que possuem uma seção de choque muito maior que os demais sabores. O reativamento da onda de choque recebe o nome de reaquecimento via neutrino e sua possibilidade foi descoberta através de simulações numéricas [68]. E todo o processo de explosão chama-se explosão atrasada de SN, ocorrendo aproximadamente 0.5 segundo após o "bounce".

Estas hipóteses de explosão são amplamente aceitas no meio científico, mas ressaltamos que não conhece-se completamente o processo de explosão de supernovas, pois além da falta de ocorrências deste tipo de eventos na nossa Galáxia, as simulações realizadas atualmente não estão conseguindo, com grande frequência, obter "explosões" e os resultados dependem fortemente das suposições feitas pelos pesquisadores [69, 70, 71, 72, 73].

\section{5}

\section{Espectro dos Neutrinos Provenientes de SN}

O espectro não-térmico dos neutrinos que saem da SN pode ser mostrado usando a distribuição de Fermi-Dirac, que de um modo geral e bastante comum na literatura é expresso como [74],

$$
F_{\nu_{\alpha}}^{0}(E)=\frac{1}{4 \pi D^{2}} \frac{\Phi_{\nu_{\alpha}}}{T_{\nu_{\alpha}}^{3} f_{2}\left(\eta_{\nu_{\alpha}}\right)} \frac{E^{2}}{e^{E / T_{\nu_{\alpha}}-\eta_{\nu_{\alpha}}+1}}
$$

onde D é a distância da Terra à SN, $\Phi_{\nu_{\alpha}}$ é o número total de neutrinos de sabor $\alpha$ emitidos, $E$ é a energia do neutrino, $T_{\nu_{\alpha}}$ e $\eta_{\nu_{\alpha}}$ representam, respectivamente, a temperatura efetiva e o potencial químico. A função $f_{n}\left(\eta_{\nu_{\alpha}}\right)$ é definida como

$$
f_{n}\left(\eta_{\nu_{\alpha}}\right) \equiv \int_{0}^{\infty} \frac{x^{n}}{\mathrm{e}^{x-\eta_{\nu_{\alpha}}}+1} \mathrm{~d} x
$$

Há outros modos de mostrar o espectro dos neutrinos como, por exemplo, 
o utilizado no trabalho de [75], que assumiu a distribuição na forma [76]

$$
F_{\nu_{\alpha}}^{0}(E)=\frac{1}{4 \pi D^{2}} \frac{\Phi_{\nu_{\alpha}}}{\left\langle E_{\nu_{\alpha}}\right\rangle} \frac{\beta_{\alpha}^{\beta_{\alpha}}}{\Gamma\left(\beta_{\alpha}\right)}\left[\frac{E}{\left\langle E_{\nu_{\alpha}}\right\rangle}\right]^{\beta_{\alpha}-1} \exp \left[-\beta_{\alpha} \frac{E}{\left\langle E_{\nu_{\alpha}}\right\rangle}\right]
$$

onde $\left\langle E_{\nu_{\alpha}}\right\rangle$ é a energia média do neutrino de sabor $\alpha, \Gamma\left(\beta_{\alpha}\right)$ é a função gama e $\beta_{\alpha}$ é um parâmetro para descrever o desvio em relação a um espectro térmico e varia tipicamente entre 2.5 e 5 .

Neste trabalho utilizaremos a eq. (2.48) para estudar o espectro de neutrinos na Terra. Esta parametrização é baseada em simulações numéricas pelo grupo de Garching [77, 78, 79] e não leva em conta a oscilação de neutrinos.

\section{6}

\section{Modelo de Distribuição de SN na Galáxia}

Neste trabalho usamos o mesmo modelo de distribuição de SN galácticas dado na Ref. [80]. Para projetar esta distribuição no céu, i.e., vista do solo, o movimento de rotação da Terra causa um efeito adicional, que é contornado ao considerarmos uma distribuição suave com simetria azimutal. Assim, podemos escrever a densidade superficial de eventos de SN local usando a parametrização usada para estrelas de nêutrons [81],

$$
\sigma_{\mathrm{SN}}(r) \propto r^{4} \exp \left[-\frac{r}{1.25 \mathrm{kpc}}\right]
$$

onde $\sigma_{S N}(r)$ é a densidade superficial de eventos de SN em função da distância radial do centro da Galáxia e pc significa parsec ${ }^{15}$. A distribuição vertical de SN é

$$
R_{\mathrm{SN}}(z) \propto 0.79 \exp \left[-\left(\frac{z}{212 \mathrm{pc}}\right)^{2}\right]+0.21 \exp \left[-\left(\frac{z}{636 \mathrm{pc}}\right)^{2}\right]
$$

onde z é a distância do plano galáctico tal que a distribuição de SN é

$$
n_{S N}(r, z) \propto R_{\mathrm{SN}}(z) \sigma_{\mathrm{SN}}(r)
$$

${ }^{15}$ Parsec é uma medida de distância usada em astronomia e é definida como a distância do Sol para um objeto astronômico cujo paralaxe é de um arco de segundo. Seu valor é:

$$
1 \mathrm{pc}=3.08567758 \times 10^{16} \mathrm{~m} .
$$




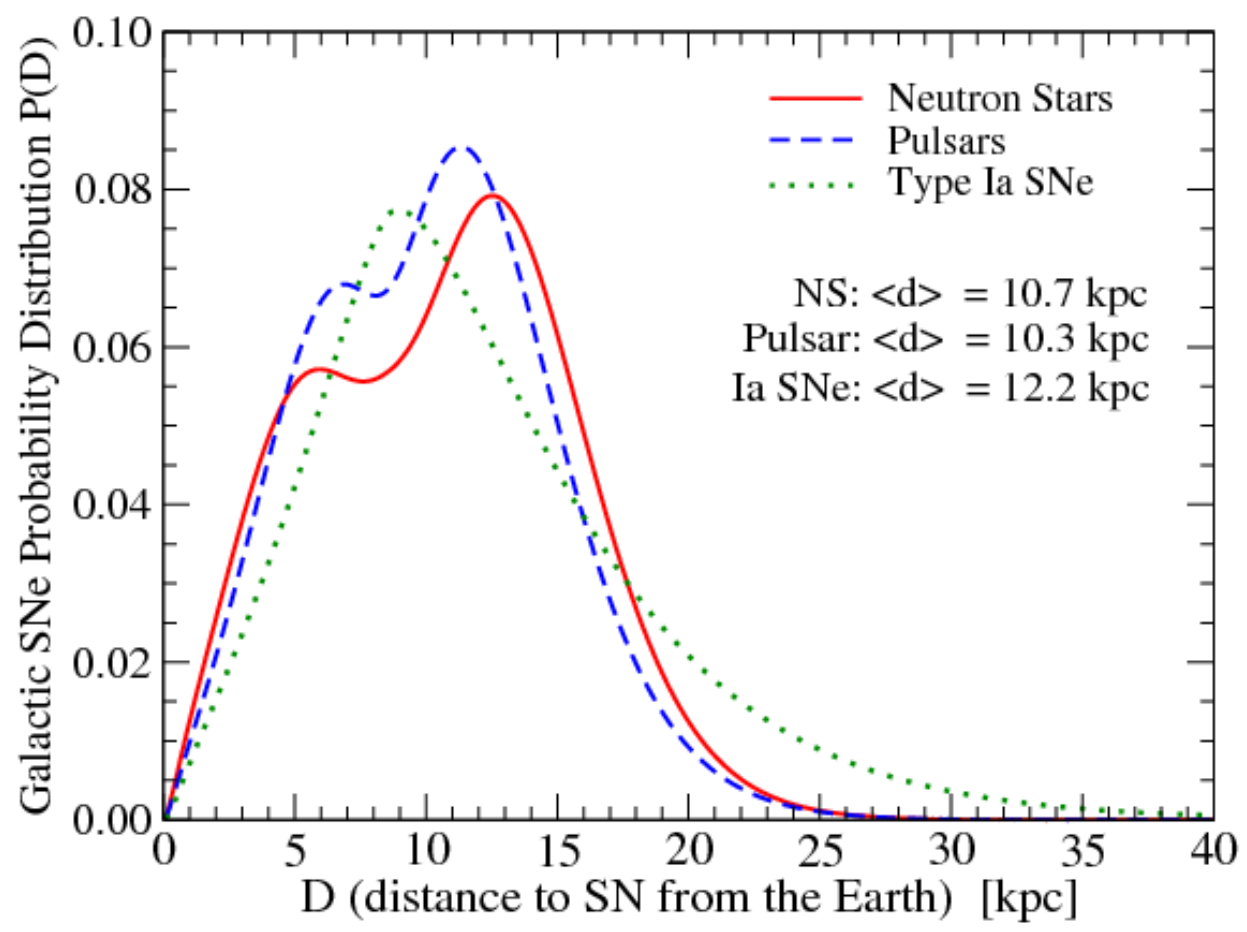

Figura 2.9: Probabilidade de distribuição de SN na Via Láctea. As linhas sólida (vermelha), tracejada (azul) e pontilhada (verde) indicam estrelas de nêutrons, pulsares e SN IA, respectivamente.

A distribuição de probabilidade de SN em função da distância à Terra esperada aqui na Via Láctea é mostrada na Fig. 2.9 [80], onde também estão representadas as distribuições para pulsares (curva tracejada) e SN IA (curva pontilhada). A normalização foi feita de forma que

$$
\int d \alpha \int \cos \delta d \delta f(\alpha, \delta)=1
$$

Utilizando esta probabilidade de distribuição, mostramos na Fig. 2.10 [82] que a maior probabilidade recai sobre o disco galáctico. Também pode ser visto nesta figura as últimas 10 SN registradas na Via Láctea juntamente com a SN1987A, que é a única observada através de neutrinos até hoje.

\section{7}

\section{SN1987A}

A única supernova estudada até o momento através de neutrinos ocorreu em fevereiro de 1987, em uma galáxia satélite à Via-Láctea denominada Grande Nuvem de Magalhães (LMC, em inglês) que situa-se a distância de 52 kpc 


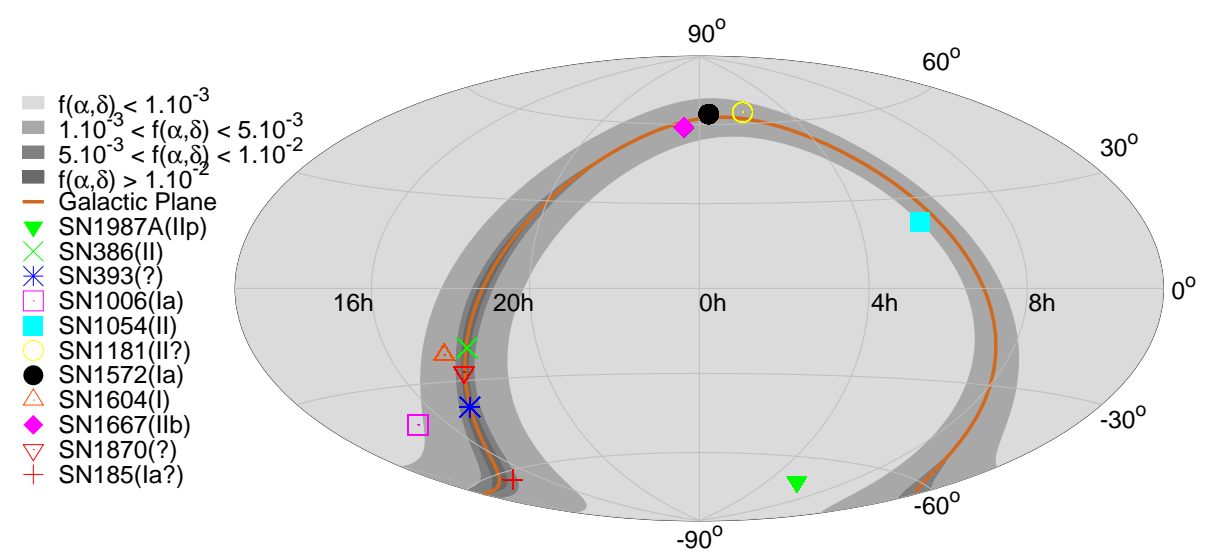

Figura 2.10: Distribuição de probabilidade de SN em coordenadas equatoriais. Maiores probabilidades "caem" sobre o disco galáctico. Os pontos são as últimas 10 SN galácticas ocorridas na Via Láctea e a SN1987A, ocorrida na Grande Nuvem de Magalhães.

da Terra. O espectro mostrou linhas de Hidrogênio [83], possibilitando sua classificação como supernova do Tipo II. Tal enquadramento, define que a explosão se deu por colapso do núcleo. Até hoje não foi possível identificar se houve remanescente da estrela progenitora, mas há indícios de que existe um pulsar [84] naquele local.

Nesta época, havia em funcionamento quatro grandes detectores subterrâneos com capacidade para perceber os neutrinos oriundos de uma SN: Kamiokande-II [85, 86], IMB [87, 88], Baksan [89] e LSD [90]. Todos os detectores registraram a passagem de neutrinos provenientes da SN1987A durante um intervalo de tempo de aproximadamente 10 segundos a algumas horas antes da possibilidade de observação ótica. Os três primeiros observaram ao mesmo tempo (com incertezas de calibração do tempo), mas o sinal do LSD foi captado cinco horas antes dos outros experimentos o que faz com que normalmente seus registros não sejam levados em consideração para análise dos dados da SN1987A [38].

Kamiokande-II e IMB são detectores de água Cherenkov e foram construídos para observarem o decaimento do próton. O detector Baksan, por sua vez, é do tipo cintilador e foi construído para observar colapsos estelares. O Kamiokande-II (IMB, Baksan) pode detectar antineutrinos eletrônicos com energia maior que $1.8 \mathrm{MeV}(20 \mathrm{MeV}, 10 \mathrm{MeV})$ através da reação do decai- 
mento beta inverso

$$
\bar{\nu}_{e}+p \rightarrow n+e^{+} .
$$

O Kamiokande-II (IMB - Baksan) reportou 11 (8 - 5) eventos devido a neutrinos da SN1987A, como mostra a tabela 2.1, reproduzida da Ref. [91]. 


\begin{tabular}{|c|c|c|c|c|}
\hline Eventos & $t_{i}(\mathrm{~s})$ & $\bar{E}_{i}(\mathrm{MeV})$ & $\overline{\sigma_{i}(\mathrm{MeV})}$ & $\overline{\overline{B_{i}\left(\mathrm{~s}^{-1}\right)}}$ \\
\hline \multicolumn{5}{|c|}{ Kamiokande-II } \\
\hline 1 & $\equiv 0.0$ & 20 & 2.9 & $1.6 \times 10^{-5}$ \\
\hline 2 & 0.107 & 13.5 & 3.2 & $1.9 \times 10^{-3}$ \\
\hline 3 & 0.303 & 7.5 & 2.0 & $2.9 \times 10^{-2}$ \\
\hline 4 & 0.324 & 9.2 & 2.7 & $1.2 \times 10^{-2}$ \\
\hline 5 & 0.507 & 12.8 & 2.9 & $2.1 \times 10^{-3}$ \\
\hline $6^{*}$ & 0.686 & 6.3 & 1.7 & $3.7 \times 10^{-2}$ \\
\hline 7 & 1.541 & 35.4 & 8.0 & $4.5 \times 10^{-5}$ \\
\hline 8 & 1.728 & 21.0 & 4.2 & $8.2 \times 10^{-5}$ \\
\hline 9 & 1.915 & 19.8 & 3.2 & $1.5 \times 10^{-5}$ \\
\hline 10 & 9.219 & 8.6 & 2.7 & $1.5 \times 10^{-2}$ \\
\hline 11 & 10.433 & 13.0 & 2.6 & $1.9 \times 10^{-3}$ \\
\hline 12 & 12.439 & 8.9 & 1.9 & $1.6 \times 10^{-2}$ \\
\hline $13^{*}$ & 17.641 & 6.5 & $1.6^{* *}$ & $3.8 \times 10^{-2}$ \\
\hline $14^{*}$ & 20.257 & 5.4 & $1.4^{* *}$ & $2.9 \times 10^{-2}$ \\
\hline $15^{*}$ & 21.355 & 4.6 & $1.3^{* *}$ & $2.8 \times 10^{-2}$ \\
\hline $16^{*}$ & 23.814 & 6.5 & $1.6^{* *}$ & $3.8 \times 10^{-2}$ \\
\hline \multicolumn{5}{|c|}{ IMB } \\
\hline 1 & $\equiv 0.0$ & 38 & 7 & 0 \\
\hline 2 & 0.412 & 37 & 7 & 0 \\
\hline 3 & 0.650 & 28 & 6 & 0 \\
\hline 4 & 1.141 & 39 & 7 & 0 \\
\hline 5 & 1.562 & 36 & 9 & 0 \\
\hline 6 & 2.684 & 36 & 6 & 0 \\
\hline 7 & 5.010 & 19 & 5 & 0 \\
\hline 8 & 5.582 & 22 & 5 & 0 \\
\hline \multicolumn{5}{|c|}{ Baksan } \\
\hline 1 & $\equiv 0.0$ & 12.0 & 2.4 & $8.4 \times 10^{-4}$ \\
\hline 2 & 0.435 & 12,9 & 3.6 & $1.3 \times 10^{-3}$ \\
\hline 3 & 1.710 & 23.5 & 4.7 & $1.2 \times 10^{-3}$ \\
\hline 4 & 7.687 & 17.6 & 3.5 & $1.3 \times 10^{-3}$ \\
\hline 5 & 9.099 & 20.3 & 4.1 & $1.3 \times 10^{-3}$ \\
\hline
\end{tabular}

* Considerado como evento de background.

**Calculado usando um ajuste linear $\sigma_{i} v s \epsilon_{i}$ para eventos anteriores.

Tabela 2.1: Eventos de neutrinos detectados nos experimentos Kamiokande-II, IMB e Baksan relativos a SN1987A. A primeira coluna indica a ordem dos eventos, a segunda coluna mostra o tempo de detecção, considerando o primeiro evento como tempo zero. A terceira informa-nos a energia do neutrino incidente e a quarta coluna nos diz a incerteza para a energia e, finalmente, a última coluna fornece a taxa média de ruído para cada evento. 


\section{3}

\section{Oscilações de Neutrinos}

A oscilação de neutrinos é um fenômeno quântico, proposto pela primeira vez por Pontecorvo [92, 93], quando este sugeriu a oscilação entre $\nu \rightarrow \bar{\nu}$ fazendo analogia com a oscilação dos Káons $\left(K^{0} \leftrightarrow \bar{K}^{0}\right)$ durante a década de 50. Tal tipo de oscilação acabou não sendo comprovada, mas a ideia de oscilação permaneceu viva e foi usada para introduzir o conceito de mistura de sabores por Maki, Nakagawa e Sakata [94] em 1962. Apesar de muito mencionada nos anos 60 e início dos 70 como solução para o problema dos neutrinos solares, foi somente na segunda metade da década de 70 que surgiu a teoria padrão para a oscilação dos neutrinos [95, 96, 97].

\section{1 \\ Neutrinos}

Neutrinos, assim como todas as partículas que formam a matéria conhecida, são férmions, o que significa que eles respeitam o Princípio de Exclusão de Pauli e obedecem a estatística de Fermi-Dirac. Também é característica geral o valor do spin $(1 / 2)$ e a helicidade ${ }^{1}$, sempre negativa para os neutrinos e sempre positiva para os antineutrinos (Fig. 3.1), considerando-os ultra-relativísticos. Mas os neutrinos estão em um subgrupo, chamado de léptons ${ }^{2}$. As partículas que pertencem a esta categoria são: elétrons, múons, taus e os respectivos neutrinos, $\nu_{e}, \nu_{\mu}$ e $\nu_{\tau}$, onde o subíndice indica o que, na física de partículas, chamamos de sabor. Cada lépton é associado a um anti-lépton, isto é, sua antipartícula (ver Tabela 3.1). Os elétrons, múons e taus possuem carga e massa enquanto que os neutrinos, por sua vez, não possuem carga e interagem muito fracamente com a matéria, somente via interação fraca - de corrente carregada

${ }^{1}$ Pode-se definir helicidade como a projeção do spin ao longo do momento, se tem mesmo sentido, é positiva; se contrários, é negativa.

${ }^{2}$ Proveniente do grego, significa leve. O nome foi dado para contrapor-se aos hádrons, que são partículas pesadas. 


\begin{tabular}{|c|c|c|c|c|c|}
\hline \multicolumn{3}{|c|}{ Léptons } & \multicolumn{3}{c|}{ Anti-Léptons } \\
\hline $1^{a}$ Geração & $2^{a}$ Geração & $3^{a}$ Geração & $1^{a}$ Geração & $2^{a}$ Geração & $3^{a}$ Geração \\
\hline$e^{-}$ & $\mu^{-}$ & $\tau^{-}$ & $e^{+}$ & $\mu^{+}$ & $\tau^{+}$ \\
$\nu_{e}$ & $\nu_{\mu}$ & $\nu_{\tau}$ & $\bar{\nu}_{e}$ & $\bar{\nu}_{\mu}$ & $\bar{\nu}_{\tau}$ \\
\hline
\end{tabular}

Tabela 3.1: Tabela de léptons e anti-léptons

(CC, em inglês) ou de corrente neutra (NC, em inglês). Por isso, os $\nu_{e}, \nu_{\mu}$ e $\nu_{\tau}$ são auto-estados da interação fraca, o que significa que são auto-estados de sabor. Por outro lado, podemos considerar os auto-estados de massa do Hamiltoniano livre e denominamos de auto-estados de massa, cujo símbolo é $\nu_{i} \mathrm{e}$ tem massa definida $m_{i}$, onde $i=1,2$ ou 3. O caso dos neutrinos é semelhante ao setor dos quarks: os auto-estados de sabor são combinações lineares dos auto-estados de massa, determinados pela matriz unitária Cabbibo-KobayashiMaskawa $(\mathrm{CKM})^{3}$ [98]. Para um aprofundamento no assunto, recomendamos a leitura, por exemplo, de [99, 100, 101]. Em outras palavras, os neutrinos também se misturam significando que um $\nu_{e}$, em princípio, é uma combinação linear dos auto-estados de massa $\nu_{i}$. Importante observar que os experimentos de oscilação de neutrinos são capazes de detectar apenas os sabores dos neutrinos, que não são auto-estados de massa do Hamiltoniano, levando à possibilidade de oscilação de neutrinos.

Meto-Disentel

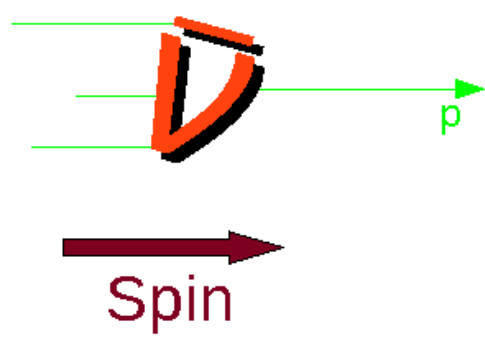

Mắ-Escuerde

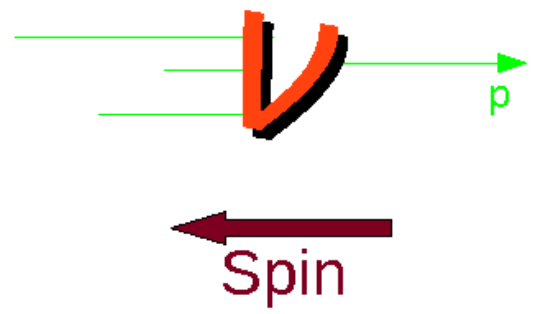

Figura 3.1: Ilustração representativa da helicidade dos neutrinos.

\footnotetext{
${ }^{3}$ Trabalho realizado primeiramente pelo físico italiano Nicola Cabbibo, em 1963, para duas gerações. Posteriormente, os físicos japoneses Makoto Kobayashi e Toshihide Maskawa ampliaram para três gerações. Tal trabalho rendeu o Prêmio Nobel de Física de 2008 aos japoneses.
} 


\subsection{1}

\section{Hierarquia}

Vimos que temos três tipos de neutrinos que são auto-estados de massa do Hamiltoniano $\left(\nu_{1}, \nu_{2}\right.$ e $\left.\nu_{3}\right)$ e embora não saibamos quais sejam os valores de cada neutrino massivo, sabemos que eles possuem valores distintos ${ }^{4}$ e, ao menos, dois deles não nulos. Há duas formas de organizar os neutrinos quanto ao seu valor absoluto de massa (veja Fig. 3.2):

1. Hierarquia Normal: é a situação quando consideramos que $m_{1}<m_{2}<$ $m_{3}$, isto é, o neutrino mais leve é $\nu_{1}$ e o mais pesado o $\nu_{3}$.

2. Hierarquia Invertida: aqui o neutrino mais leve é $\nu_{3}$ e o mais pesado o $\nu_{2}$, resultando em $m_{2}>m_{1}>m_{3}$.

\section{2 \\ Oscilação no Vácuo}

\subsection{1}

\section{Neutrinos}

Na teoria padrão para a oscilação, um neutrino com sabor $\alpha$ e momento $\vec{p}$ criado em um processo de interação fraca pode ter o seu estado de sabor descrito como

$$
\left|\nu_{\alpha}\right\rangle=\sum_{k} U_{\alpha k}^{*}\left|\nu_{k}\right\rangle \quad(\alpha=e, \mu, \tau)
$$

onde U é a matriz de mistura, unitária ${ }^{5}$, para o setor de neutrinos. Ela foi batizada de Matriz $\mathrm{MNS}^{6}$ em homenagem aos físicos que a criaram, Maki, Nakagawa e Sakata ${ }^{7}$, em 1962. Como temos três espécies de neutrinos ativos, a matriz MNS é 3 x 3 e parametrizada por rotações, semelhante as rotações

${ }^{4}$ Condição necessária para haver oscilação entre os sabores, já comprovado experimentalmente.

${ }^{5}$ É uma matriz complexa, $3 \times 3$, que satisfaz a condição

$$
U^{\dagger} U=1
$$

${ }^{6}$ A matriz MNS também é conhecida na literatura como matriz PMNS em referência ao trabalho pioneiro realizado por Pontecorvo.

${ }^{7}$ Originalmente o trabalho dos físicos japoneses envolveu apenas mistura para duas gerações, sendo ampliado para três alguns anos mais tarde. 


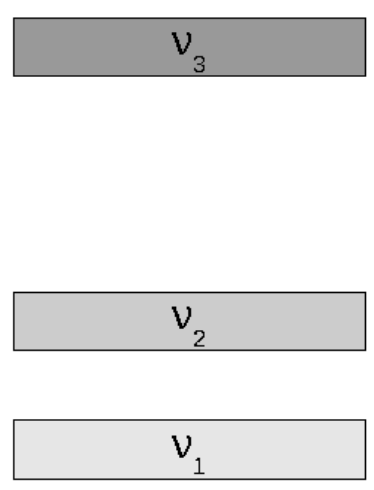

NORMAL

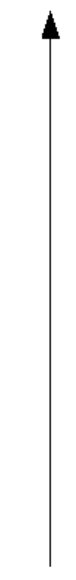

MASSA
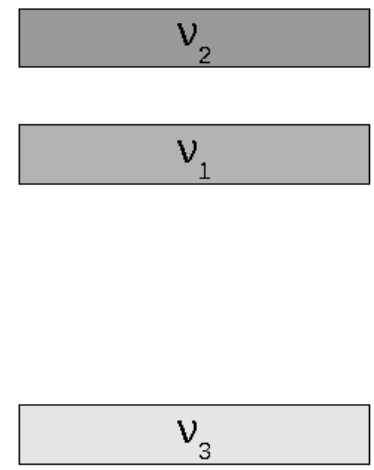

INVERTIDA

Figura 3.2: Ilustração representativa da hierarquia de massa dos neutrinos.

da mecânica utilizando ângulos de Euler. Estas rotações podem ser feitas em várias ordens, mudando sua forma final, embora não altere os resultados físicos. No entanto, iremos utilizar a mesma ordem que é amplamente vista na literatura [57],

$$
U_{M N S}=\left(\begin{array}{ccc}
1 & 0 & 0 \\
0 & c_{23} & s_{23} \\
0 & -s_{23} & c_{23}
\end{array}\right)\left(\begin{array}{ccc}
c_{13} & 0 & s_{13} e^{-i \phi} \\
0 & 1 & 0 \\
-s_{13} e^{i \phi} & 0 & c_{13}
\end{array}\right)\left(\begin{array}{ccc}
c_{12} & s_{12} & 0 \\
-s_{12} & c_{12} & 0 \\
0 & 0 & 1
\end{array}\right)
$$

que resulta na expressão

$$
U_{M N S}=\left(\begin{array}{ccc}
c_{12} c_{13} & s_{12} c_{13} & s_{13} e^{-i \phi} \\
-s_{12} c_{23}-c_{12} s_{23} s_{13} e^{i \phi} & c_{12} c_{23}-s_{12} s_{23} s_{13} e^{i \phi} & s_{23} c_{13} \\
s_{12} s_{23}-c_{12} c_{23} s_{13} e^{i \phi} & -c_{12} s_{23}-s_{12} c_{23} s_{13} e^{i \phi} & c_{23} c_{13}
\end{array}\right),
$$

onde definimos que $c_{i j}\left(s_{i j}\right)$ é $\cos \theta_{i j}\left(\sin \theta_{i j}\right)$ e os subíndices do ângulo $\theta$ indicam a mistura entre os dois neutrinos massivos envolvidos, e $\phi$ é a fase de violação carga-paridade $(\mathrm{CP})$. Para uma melhor compreensão veja a ilustração da Fig. 3.3, retirada da Ref. [102].

A título de curiosidade, a matriz MNS representada na Eq. (3.3) tem esta forma se considerarmos que neutrinos são partículas de Dirac, assim como o são todas as partículas conhecidas do Modelo Padrão. Entretanto, existe a possibilidade de neutrinos terem outra natureza, i.e., serem partículas 
de Majorana ${ }^{8}$, o que implicaria em uma modificação na matriz MNS. Esta mudança seria o acréscimo de duas fases de violação de $\mathrm{CP}$, deixando a Eq. (3.3) assim:

$$
U_{M N S}^{M}=U_{M N S} \times\left(\begin{array}{ccc}
e^{i \alpha_{1}} & 0 & 0 \\
0 & e^{i \alpha_{2}} & 0 \\
0 & 0 & 1
\end{array}\right)
$$

Mas para o estudo de oscilação, a natureza dos neutrinos não causa qualquer efeito nos resultados porque as fases de violação de CP de Majorana são canceladas durante o desenvolvimento matemático [103, 104, 105]. Sendo assim, vamos trabalhar com a forma de Dirac por simplicidade.

Para termos a ortonormalidade nos auto-estados de massa, de acordo com a Fig 3.3, assumimos que

$$
\left\langle\nu_{k} \mid \nu_{j}\right\rangle=\delta_{k j} \quad(k, j=1,2,3),
$$

e a unitariedade da matriz de mistura implica que os estados de sabores também são ortonormais

$$
\left\langle\nu_{\alpha} \mid \nu_{\beta}\right\rangle=\delta_{\alpha \beta} \quad(\alpha, \beta=e, \mu, \tau) .
$$

Como dito acima, os auto-estados do Hamiltoniano não são os estados de sabores do neutrino, mas sim, os estados de massa. Então

$$
\mathscr{H}\left|\nu_{k}\right\rangle=E_{k}\left|\nu_{k}\right\rangle
$$

onde a energia $E_{k}$ é

$$
E_{k}=\sqrt{\vec{p}^{2}+m_{k}^{2}}
$$

Utilizando a equação correspondente a equação de Schrödinger, da mecânica

${ }^{8}$ Teoria formulada pelo físico italiano Ettore Majorana na década de 1930. Basicamente, uma partícula seria também sua antipartícula, i.e., não há diferença entre uma partícula e sua antipartícula. Não conhecemos nenhum férmiom que satisfaça esta condição atualmente, mas neutrinos podem ter esta característica, por ter carga nula. O estudo do duplo decaimento beta sem neutrinos é uma das formas de provarmos a natureza do neutrino (Dirac ou Majorana). 


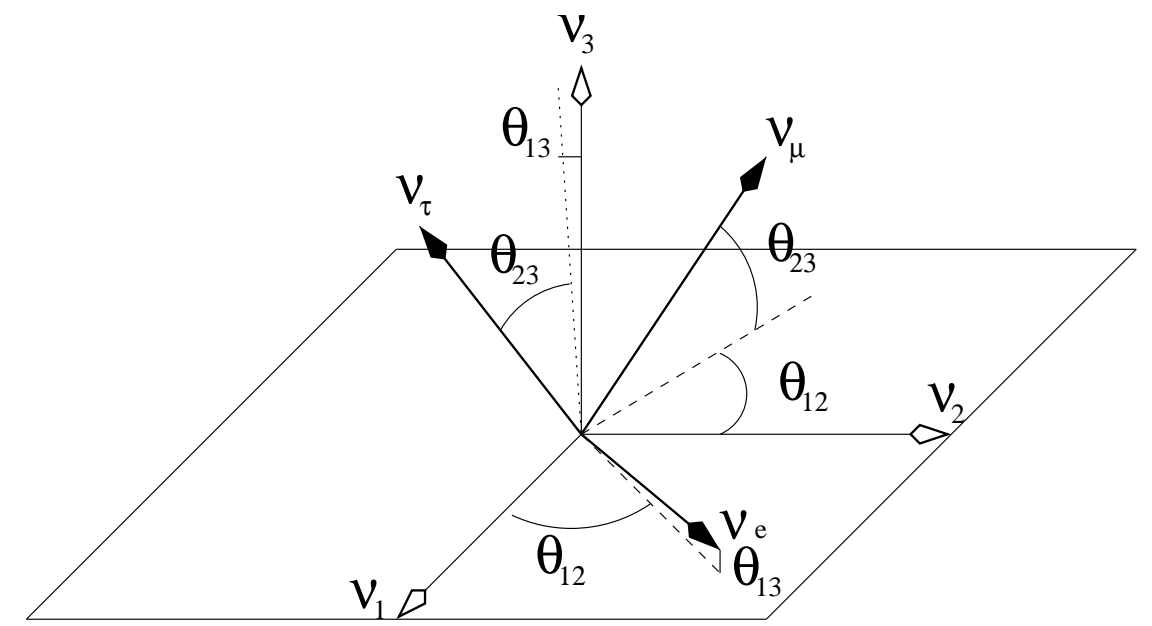

Figura 3.3: Relação entre os auto-estados de sabor dos neutrinos $\nu_{e}, \nu_{\mu}$ e $\nu_{\tau}$ e os auto-estados de massa $\nu_{1}, \nu_{2}$ e $\nu_{3}$ em termos dos ângulos de mistura $\theta_{12}, \theta_{13}, \theta_{23}$.

quântica não relativística, vemos que os neutrinos massivos evoluem no tempo,

$$
\begin{aligned}
i \frac{d}{d t}\left|\nu_{k}(t)\right\rangle & =\mathscr{H}\left|\nu_{k}(t)\right\rangle, \\
\left|\nu_{k}(t)\right\rangle & =e^{-i E_{k} t}\left|\nu_{k}\right\rangle .
\end{aligned}
$$

Considerando o estado de sabor, $\left|\nu_{\alpha}(t)\right\rangle$, que no tempo $t=0$ cria um neutrino com sabor $\alpha$ e aplicando a eq. (3.1) podemos ter a evolução temporal como

$$
\left|\nu_{\alpha}(t)\right\rangle=\sum_{k} U_{\alpha k}^{*} e^{-i E_{k} t}\left|\nu_{k}\right\rangle
$$

e usando a relação de unitariedade da matriz U podemos expressar os estados massivos em termos dos estados de sabor. Assim, a eq. (3.1) fica

$$
\left|\nu_{k}\right\rangle=\sum_{\alpha} U_{\alpha k}\left|\nu_{\alpha}\right\rangle
$$

Substituindo a eq. (3.12) na eq. (3.11), temos

$$
\left|\nu_{\alpha}(t)\right\rangle=\sum_{\beta=e, \mu, \tau}\left(\sum_{k} U_{\alpha k}^{*} e^{-i E_{k} t} U_{\beta k}\right)\left|\nu_{\beta}\right\rangle .
$$

Então, a superposição dos estados massivos resulta em um sabor, como na eq. (3.1) no instante $t=0$. Mas na medida que o tempo evolui $(t>0)$, torna-se uma superposição de diferentes estados de sabor ${ }^{9}$.

${ }^{9}$ Somente se a matriz de mistura U não for diagonal, o que indica que os neutrinos misturam-se. 
O coeficiente de $\left|\nu_{\beta}\right\rangle$ é a amplitude de transição (A) do sabor $\alpha$ para o sabor $\beta$ em função do tempo. Matematicamente, escrevemos

$$
A_{\nu_{\alpha} \rightarrow \nu_{\beta}}(t) \equiv\left\langle\nu_{\beta} \mid \nu_{\alpha}(t)\right\rangle=\sum_{k} U_{\alpha k}^{*} U_{\beta k} e^{-i E_{k} t}
$$

Sabendo a amplitude de transição, podemos determinar a probabilidade de transição $(\mathrm{P})$,

$$
P_{\nu_{\alpha} \rightarrow \nu_{\beta}}(t)=\left|A_{\nu_{\alpha} \rightarrow \nu_{\beta}}(t)\right|^{2}=\sum_{k, j} U_{\alpha k}^{*} U_{\beta k} U_{\alpha j} U_{\beta j}^{*} e^{-i\left(E_{k}-E_{j}\right) t}
$$

expandindo a eq. (3.8) temos

$$
E_{k}=|\vec{p}|\left(1+\frac{m_{k}^{2}}{\vec{p}^{2}}\right)^{1 / 2} \simeq|\vec{p}|+\frac{m_{k}^{2}}{2|\vec{p}|}
$$

e levando em conta que neutrinos são ultra-relativísticos, podemos desprezar a contribuição da massa e fazer $E=|\vec{p}|$, deixando a equação

$$
E_{k} \simeq E+\frac{m_{k}^{2}}{2 E}
$$

Para a diferença de energia da eq. (3.15) utilizamos a eq. (3.17) e obtemos

$$
E_{k}-E_{j} \simeq \frac{m_{k}^{2}-m_{j}^{2}}{2 E}=\frac{\Delta m_{k j}^{2}}{2 E}
$$

deixando a probabilidade de transição de sabor, eq. (3.15), como

$$
P_{\nu_{\alpha} \rightarrow \nu_{\beta}}(t)=\sum_{k, j} U_{\alpha k}^{*} U_{\beta k} U_{\alpha j} U_{\beta j}^{*} \exp \left(-i \frac{\Delta m_{k j}^{2} t}{2 E}\right) .
$$

Por questões práticas, vamos fazer mais uma mudança: nos experimentos de oscilação de neutrinos não se mensura o tempo de propagação e como conhecemos a distância $(L)$ entre a fonte de neutrinos (reator, acelerador, supernova, etc) e o detector, podemos fazer a aproximação $t=L$, pois lembremos que estas partículas são ultra-relativísticas, isto é, viajam com velocidades muito próximas a da luz. Assim, a probabilidade de conversão 
é dada por

$$
P_{\nu_{\alpha} \rightarrow \nu_{\beta}}(L, E)=\sum_{k, j} U_{\alpha k}^{*} U_{\beta k} U_{\alpha j} U_{\beta j}^{*} \exp \left(-i \frac{\Delta m_{k j}^{2} L}{2 E}\right)
$$

Note que assim somente a distância $L$ e a energia $E$ são as grandezas que variam nos experimentos e que determinam a fase de oscilação. A diferença de massa quadrada $\left(\Delta m_{k j}^{2}\right)$, na fase de oscilação, é uma constante física e os elementos da matriz de mistura U, que compõem a amplitude de oscilação, são constantes da natureza.

Outro ponto importante da eq. (3.20) é que se $L=0$ temos,

$$
P_{\nu_{\alpha} \rightarrow \nu_{\beta}}(L=0, E)=\sum_{k, j} U_{\alpha k}^{*} U_{\beta k} U_{\alpha j} U_{\beta j}^{*}
$$

mas a relação de unitariedade nos diz que

$$
U U^{\dagger}=1 \Longleftrightarrow \sum_{k} U_{\alpha k} U_{\beta k}^{*}=\delta_{\alpha \beta}
$$

implicando que a troca de sabor só ocorrerá se existir alguma distância, isto é, $L>0$.

A eq. (3.20) pode ser reescrita - se reorganizarmos a amplitude de oscilação - na forma

$$
P_{\nu_{\alpha} \rightarrow \nu_{\beta}}(L, E)=\sum_{k}\left|U_{\alpha k}\right|^{2}\left|U_{\beta k}\right|^{2}+2 \mathfrak{R e} \sum_{k>j} U_{\alpha k}^{*} U_{\beta k} U_{\alpha j} U_{\beta j}^{*} \exp \left(-2 \pi i \frac{L}{L_{k j}^{o s c}}\right)
$$

onde $L_{k j}^{o s c}$ é o comprimento de oscilação ${ }^{10}$,

$$
L_{k j}^{o s c}=\frac{4 \pi E}{\left|\Delta m_{k j}^{2}\right|}
$$

Para vermos outra forma amplamente usada de probabilidade de oscila-

${ }^{10}$ Comprimento de oscilação é a distância em que a fase gerada pela diferença de massa quadrada, $\Delta m_{k j}^{2}$, torna-se igual a $2 \pi$. 
ção, fazemos o quadrado da relação de unitariedade (eq. (3.22)),

$$
\sum_{k}\left|U_{\alpha k}\right|^{2}\left|U_{\beta k}\right|^{2}+2 \mathfrak{R e} \sum_{k>j}\left[U_{\alpha k}^{*} U_{\beta k} U_{\alpha j} U_{\beta j}^{*}\right]=\delta_{\alpha \beta}
$$

e rearrumamos os termos para ficar com a expressão

$$
\sum_{k}\left|U_{\alpha k}\right|^{2}\left|U_{\beta k}\right|^{2}=\delta_{\alpha \beta}-2 \Re \mathfrak{R e} \sum_{k>j}\left[U_{\alpha k}^{*} U_{\beta k} U_{\alpha j} U_{\beta j}^{*}\right]
$$

Agora, partindo novamente da eq. (3.20), trabalhando a amplitude de oscilação, separando as partes real e imaginária e usando a eq. (3.26), obtemos

$$
\begin{aligned}
P_{\nu_{\alpha} \rightarrow \nu_{\beta}}(L, E)= & \delta_{\alpha \beta}-2 \mathfrak{R e} \sum_{k>j}\left[U_{\alpha k}^{*} U_{\beta k} U_{\alpha j} U_{\beta j}^{*}\right]+ \\
& +2 \mathfrak{R e} \sum_{k>j} U_{\alpha k}^{*} U_{\beta k} U_{\alpha j} U_{\beta j}^{*} \cos \left(\frac{\Delta m_{k j}^{2} L}{2 E}\right)+ \\
& +2 \mathfrak{I m} \sum_{k>j} U_{\alpha k}^{*} U_{\beta k} U_{\alpha j} U_{\beta j}^{*} \sin \left(\frac{\Delta m_{k j}^{2} L}{2 E}\right)
\end{aligned}
$$

onde utilizamos a relação

$$
e^{-i \theta}=\cos \theta-i \sin \theta
$$

para modificarmos o termo oscilante. Rearrumando a eq. (3.27)

$$
\begin{aligned}
P_{\nu_{\alpha} \rightarrow \nu_{\beta}}(L, E)= & \delta_{\alpha \beta}-2 \sum_{k>j} \mathfrak{R e}\left[U_{\alpha k}^{*} U_{\beta k} U_{\alpha j} U_{\beta j}^{*}\right]\left[1-\cos \left(\frac{\Delta m_{k j}^{2} L}{2 E}\right)\right]+ \\
& +2 \sum_{k>j} \mathfrak{I m}\left[U_{\alpha k}^{*} U_{\beta k} U_{\alpha j} U_{\beta j}^{*}\right] \sin \left(\frac{\Delta m_{k j}^{2} L}{2 E}\right)
\end{aligned}
$$

fazendo proveito da relação trigonométrica

$$
\sin ^{2} \theta=\frac{1}{2}(1-\cos 2 \theta)
$$


podemos deixar a probabilidade de oscilação como

$$
\begin{aligned}
P_{\nu_{\alpha} \rightarrow \nu_{\beta}}(L, E)= & \delta_{\alpha \beta}-4 \sum_{k>j} \mathfrak{R e}\left[U_{\alpha k}^{*} U_{\beta k} U_{\alpha j} U_{\beta j}^{*}\right] \sin ^{2}\left(\frac{\Delta m_{k j}^{2} L}{4 E}\right)+ \\
& +2 \sum_{k>j} \mathfrak{I m}\left[U_{\alpha k}^{*} U_{\beta k} U_{\alpha j} U_{\beta j}^{*}\right] \sin \left(\frac{\Delta m_{k j}^{2} L}{2 E}\right)
\end{aligned}
$$

Então, esta é a forma geral da probabilidade de oscilação e pode ser dividida em dois casos: se considerarmos o canal $\alpha \neq \beta$ passamos a chamá-la de probabilidade de transição, enquanto que se considerarmos $\alpha=\beta$, denominamos probabilidade de sobrevivência. Neste último caso pode-se perceber que os componentes da amplitude de oscilação (elementos da matriz de mistura) não têm parte imaginária, uma vez que sua forma é $\left|U_{\alpha k}\right|^{2}\left|U_{\alpha j}\right|^{2}$ e o último termo da eq. (3.31) desaparece e fica com a seguinte aparência:

$$
P_{\nu_{\alpha} \rightarrow \nu_{\alpha}}(L, E)=1-4 \sum_{k>j} \mathfrak{R e}\left|U_{\alpha k}\right|^{2}\left|U_{\alpha j}\right|^{2} \sin ^{2}\left(\frac{\Delta m_{k j}^{2} L}{4 E}\right)
$$

\section{2 .2}

\section{Antineutrinos}

Assim como no caso dos neutrinos, os antineutrinos com sabor $\alpha$ ( $\alpha=$ $e, \mu, \tau)$ são comumente produzidos em interações fracas pelo canal CC. Os antineutrinos $\bar{\nu}_{\alpha}$ são superposições de antineutrinos massivos $\bar{\nu}_{k}(k=1,2,3)$,

$$
\left|\bar{\nu}_{\alpha}\right\rangle=\sum_{k} U_{\alpha k}\left|\bar{\nu}_{k}\right\rangle
$$

onde U é a matriz de mistura dos antineutrinos. Destacamos a semelhança entre esta equação e a eq. (3.1), onde podemos ver que a única diferença entre ambas expressões se dá no coeficiente dos neutrinos (antineutrinos) massivos que sofre uma conjugação complexa.

As propriedades cinemáticas dos antineutrinos são exatamente iguais as dos neutrinos, por isso o desenvolvimento matemático feito acima para o caso de neutrinos é válido aqui também, com exceção do complexo conjugado da matriz de mistura. Então, a probabilidade de transição de sabor do 
antineutrino é

$$
P_{\bar{\nu}_{\alpha} \rightarrow \bar{\nu}_{\beta}}(L, E)=\sum_{k, j} U_{\alpha k} U_{\beta k}^{*} U_{\alpha j}^{*} U_{\beta j} \exp \left(-i \frac{\Delta m_{k j}^{2} L}{2 E}\right)
$$

Repare no termo oscilatório, veja que ele é idêntico ao caso dos neutrinos. Isto indica que o comprimento de oscilação $\left(L_{k j}^{o s c}\right)$ não muda para os antineutrinos. Realizando o mesmo trabalho matemático de separar as partes real e imaginária da amplitude de oscilação e usufruindo novamente das relações de trigonometria, a probabilidade de oscilação do antineutrino é

$$
\begin{aligned}
P_{\bar{\nu}_{\alpha} \rightarrow \bar{\nu}_{\beta}}(L, E)=\delta_{\alpha \beta} & -4 \sum_{k>j} \mathfrak{R e}\left[U_{\alpha k}^{*} U_{\beta k} U_{\alpha j} U_{\beta j}^{*}\right] \sin ^{2}\left(\frac{\Delta m_{k j}^{2} L}{4 E}\right) \\
& -2 \sum_{k>j} \mathfrak{I m}\left[U_{\alpha k}^{*} U_{\beta k} U_{\alpha j} U_{\beta j}^{*}\right] \sin \left(\frac{\Delta m_{k j}^{2} L}{2 E}\right),
\end{aligned}
$$

e difere-se do caso anterior apenas pelo sinal do último termo, a parte imaginária dos elementos da matriz de mistura.

\section{3}

\section{Mistura para Duas Gerações}

Nesta seção veremos uma aproximação muito usada, a mistura para duas gerações. Vamos desconsiderar um terceiro neutrino, tanto como sabor, como em massa, o que torna os cálculos muito mais simples e dependentes de menos parâmetros que o caso de três neutrinos. Outro argumento a favor desta aproximação é o fato de que, experimentalmente, muitas vezes não somos capazes de distinguir a influência da mistura dos três neutrinos ativos e os dados são analisados com um modelo cuja mistura é somente entre dois neutrinos.

Então, sejam dois neutrinos de sabores $\nu_{\alpha}$ e $\nu_{\beta}$, onde cada sabor é uma combinação linear de dois neutrinos massivos, $\nu_{1}$ e $\nu_{2}$. Antes de prosseguirmos, vamos destacar dois pontos importantes:

1. Os sabores $\alpha e \beta$ são sabores puros, isto é, $\alpha, \beta=e, \mu$ ou $\alpha, \beta=\mu, \tau$ ou ainda $\alpha, \beta=e, \tau$; ou

2. Os sabores $\alpha$ e $\beta$ são combinações lineares de sabores puros como, por exemplo, $\nu_{\alpha}=\nu_{e}$ e $\nu_{\beta}=c_{\mu} \nu_{\mu}+c_{\tau} \nu_{\tau} \operatorname{com} c_{\mu}^{2}+c_{\tau}^{2}=1$. Esta condição 
é muito utilizada nos experimentos de desaparecimento de neutrinos eletrônicos.

A matriz de mistura efetiva é

$$
U=\left(\begin{array}{cc}
\cos \theta & \sin \theta \\
-\sin \theta & \cos \theta
\end{array}\right)
$$

onde $\theta$ é o ângulo de mistura, cujo valor encontra-se no intervalo $0 \leq \theta \leq \pi / 2$.

Para calcularmos a probabilidade de transição para $\alpha \neq \beta$, usamos a eq. (3.31). O primeiro e o último termo desaparecem, restando apenas o segundo, cuja amplitude de oscilação pode ser encontrada através da eq. (3.36)

$$
\begin{aligned}
P_{\nu_{\alpha} \rightarrow \nu_{\beta}}(L, E) & =-4[-\sin \theta \cos \theta \cos \theta \sin \theta] \sin ^{2}\left(\frac{\Delta m^{2} L}{4 E}\right) \\
& =4 \sin ^{2} \theta \cos ^{2} \theta \sin ^{2}\left(\frac{\Delta m^{2} L}{4 E}\right) \\
& =\sin ^{2} 2 \theta \sin ^{2}\left(\frac{\Delta m^{2} L}{4 E}\right) \quad(\alpha \neq \beta),
\end{aligned}
$$

onde utilizamos a relação trigonométrica $\sin 2 \theta=2 \sin \theta \cos \theta$. Olhando para o termo oscilante, vemos que a diferença de massa quadrada não possui subíndices, isto porque estamos trabalhando com duas gerações de neutrinos:

$$
\Delta m^{2} \equiv \Delta m_{21}^{2} \equiv m_{2}^{2}-m_{1}^{2}
$$

e, por simplicidade de cálculo, fazemos $m_{1}$ o mais leve, levando $\Delta m^{2}$ a ser positivo.

Para o caso de $\alpha=\beta$, a probabilidade de sobrevivência provém da eq. $(3.32)$,

$$
P_{\nu_{\alpha} \rightarrow \nu_{\alpha}}(L, E)=1-\sin ^{2} 2 \theta \sin ^{2}\left(\frac{\Delta m^{2} L}{4 E}\right) \quad(\alpha=\beta)
$$

Da ausência de qualquer fase na matriz de mistura efetiva para dois neutrinos (eq. 3.36) vemos que não há violação Carga-Paridade (CP), levando a igualdade na probabilidade de transição entre neutrinos e antineutrinos:

$$
P_{\nu_{\alpha} \rightarrow \nu_{\beta}}(L, E)=P_{\nu_{\beta} \rightarrow \nu_{\alpha}}(L, E)=P_{\bar{\nu}_{\alpha} \rightarrow \bar{\nu}_{\beta}}(L, E)=P_{\bar{\nu}_{\beta} \rightarrow \bar{\nu}_{\alpha}}(L, E)
$$




\section{4}

\section{Oscilação de Neutrinos na Matéria}

Quando neutrinos propagam-se na matéria estão sujeitos a um potencial $^{11}$ devido ao espalhamento elástico coerente do neutrino com os elétrons e nucleons do meio [106], alterando a mistura dos neutrinos. Os neutrinos também estão sujeitos a espalhamentos incoerentes, cuja ordem de magnitude do livre caminho médio pode ser estimada sem dificuldades, sabendo que

$$
\sigma_{c m} \sim G_{F} s
$$

onde s é a variável de Mandelstam ${ }^{12}$ e $G_{F}$ é a constante de Fermi, cujo valor é $G_{F}=1.166 \times 10^{-5} \mathrm{GeV}^{-2}$. A variável de Mandelstan representa o quadrado da energia total no referencial do centro de massa. No referencial do laboratório (alvo em repouso) temos $s=2 E M$, com E sendo a energia do neutrino e $\mathrm{M}$ a massa do alvo.

$$
\sigma_{l a b} \sim G_{F} E M \sim 10^{-38} \frac{E M}{\mathrm{GeV}^{2}} \quad \mathrm{~cm}^{2}
$$

e o caminho livre médio no meio

$$
\ell \sim \frac{1}{N \sigma} \sim \frac{10^{38} \mathrm{~cm}}{\left(N \mathrm{~cm}^{3}\right)\left(E M / \mathrm{GeV}^{2}\right)}
$$

sendo $\mathrm{N}$ a densidade do número de partículas alvo, normalmente nucleons. Para termos uma idéia do resultado, na matéria normal a densidade é $N_{A} / \mathrm{cm}^{3} \sim$ $10^{24} \mathrm{~cm}^{-3}$, gerando

$$
\ell \sim \frac{10^{14}}{E / \mathrm{GeV}} \mathrm{cm}
$$

Repare que a Terra $\left(R_{\oplus} \sim 10^{9} \mathrm{~cm}\right)$ só seria uma barreira para os neutrinos com energia superior a $10^{5} \mathrm{GeV}$ (um valor muito elevado!) ou para regiões onde a densidade fosse extremamente alta, por exemplo $10^{12} N_{A} \cdot \mathrm{cm}^{-3}$, onde os neutrinos com energia típica da ordem de $\mathrm{MeV}$ teriam um livre caminho médio de poucos quilômetros. Tais densidades são alcançadas em estrelas de

\footnotetext{
${ }^{11}$ Esse potencial é equivalente ao índice de refração, que tem seu valor de acordo com a velocidade da luz no meio.

${ }^{12}$ São quantidades numéricas que englobam energia, momento e os ângulos de espalhamento de partículas em processos de colisões de duas para duas partículas $p_{1}+p_{2} \rightarrow p_{3}+p_{4}$
} 
nêutrons e núcleos de supernovas.

\subsection{1}

\section{Potencial Efetivo na Matéria}

O comportamento do neutrino muda quando ele se propaga na matéria. Sua evolução é afetada pelo potencial efetivo das interações fracas de CC e NC (Fig. 3.4). Pela interação fraca de CC, temos o potencial $V_{C C}$ para um neutrino eletrônico propagando-se em um meio homogêneo e isotrópico de elétrons não polarizados [106], como mostraremos abaixo.

Da Lagrangeana efetiva de interação fraca por corrente carregada em baixa energia [38] obtemos a Hamiltoniana efetiva de CC,

$$
\mathscr{H}_{e f}^{(C C)}(x)=\frac{G_{F}}{\sqrt{2}}\left[\bar{\nu}_{e}(x) \gamma^{\rho}\left(1-\gamma^{5}\right) e(x)\right]\left[\bar{e}(x) \gamma_{\rho}\left(1-\gamma^{5}\right) \nu_{e}(x)\right]
$$

e para separarmos as contribuições do neutrino e do elétron, aplicamos a Transformação de Fierz, dada por

$$
\mathscr{H}_{e f}^{(V-A)}\left(\psi_{1}, \psi_{2}, \psi_{3}, \psi_{4}\right)=\mathscr{H}_{e f}^{(V-A)}\left(\psi_{1}, \psi_{4}, \psi_{3}, \psi_{2}\right)
$$

na Eq. (3.45) e o resultado será

$$
\mathscr{H}_{e f}^{(C C)}(x)=\frac{G_{F}}{\sqrt{2}}\left[\bar{\nu}_{e}(x) \gamma^{\rho}\left(1-\gamma^{5}\right) \nu_{e}(x)\right]\left[\bar{e}(x) \gamma_{\rho}\left(1-\gamma^{5}\right) e(x)\right]
$$

Fazendo a média do Hamiltoniano efetivo sobre o fundo de elétrons no referencial de repouso do meio, temos que

$$
\begin{aligned}
\overline{\mathscr{H}_{e f}^{(C C)}}(x)= & \frac{G_{F}}{\sqrt{2}} \bar{\nu}_{e}(x) \gamma^{\rho}\left(1-\gamma^{5}\right) \nu_{e}(x) \int d^{3} p_{e} f\left(E_{e}, T\right) \\
& \times \frac{1}{2} \sum_{h_{e}= \pm 1}\left\langle e^{-}\left(p_{e}, h_{e}\right)\left|\bar{e}(x) \gamma_{\rho}\left(1-\gamma^{5}\right) e(x)\right| e^{-}\left(p_{e}, h_{e}\right)\right\rangle,
\end{aligned}
$$

onde $f\left(E_{e}, T\right)$ é uma função distribuição e os estados do elétron correspondem ao diagrama da esquerda na Fig. 3.4. Note que o quadri-momento e a helicidade não mudam com o espalhamento porque a interação não deve modificar o meio. Vamos normalizar os estados do elétron a um volume finito $(V)$ e a integral em que a distribuição estatística depende da energia do elétron, $E_{e}$, e da temperatura $T$, para $N_{e} V$ (densidade eletrônica e volume, respectivamente), 

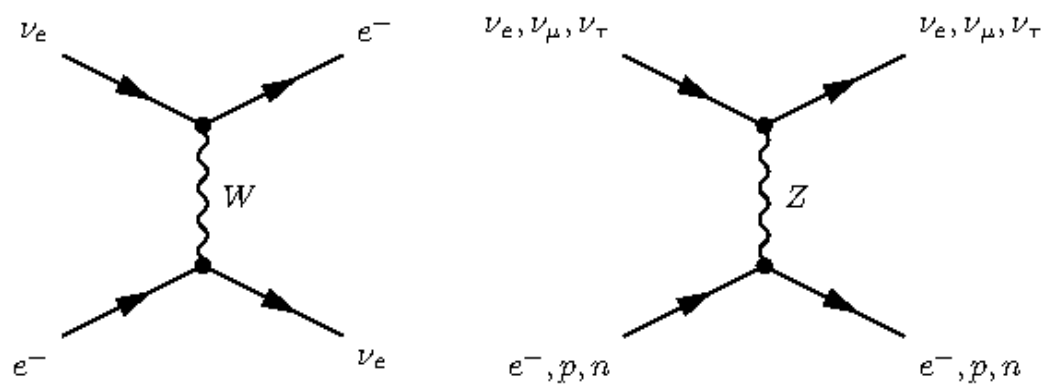

Figura 3.4: Diagramas de Feynman representando processos de espalhamento elástico coerente que geram os potenciais $V_{C C}$, através da troca de um bóson W, e $V_{N C}$, pela troca de um bóson $\mathrm{Z}$.

que é o número total de elétrons. O resultado da média da Hamiltoniana é

$$
\overline{\mathscr{H}_{e f}^{(C C)}}(x)=V_{C C} \bar{\nu}_{e}(x) \gamma^{0} \nu_{e}(x)
$$

sendo o potencial de corrente carregada $\left(V_{C C}\right)$ na forma

$$
V_{C C}=\sqrt{2} G_{F} N_{e}
$$

onde $N_{e}$ é o número de densidade de elétrons.

Para o potencial NC de neutrinos propagando-se em um meio com densidade fermiônica $N_{f}$, o cálculo é feito de maneira análoga: partindo da Lagrangeana efetiva de interação fraca de corrente neutra encontramos a Hamiltoniana efetiva

$$
\begin{aligned}
\overline{\mathscr{H}_{e f}^{(N C)}}(x)= & \frac{G_{F}}{\sqrt{2}} \sum_{\alpha=e, \mu, \tau}\left[\bar{\nu}_{\alpha}(x) \gamma^{\rho}\left(1-\gamma^{5}\right) \nu_{\alpha}(x)\right] \times \\
& \sum_{f}\left[\bar{f}(x) \gamma_{\rho}\left(g_{V}^{f}-g_{A}^{f} \gamma^{5}\right) f(x)\right],
\end{aligned}
$$

mas agora trabalha-se com todos os sabores, uma vez que o meio interage igualmente com todos os neutrinos, independentemente de sabor. O potencial de corrente neutra, $V_{N C}$, que encontra-se é

$$
V_{N C}^{f}=\sqrt{2} G_{F} N_{f} g_{V}^{f}
$$

onde $g_{V}^{f}$ é a constante de acoplamento fraco para férmions (quarks, neutrinos e léptons carregados) e cujos valores encontram-se na Tab. 3.2. Mantendo-se a idéia de um meio eletricamente neutro, consideramos que há um número 


\begin{tabular}{cc}
\hline Fermions & $g_{V}$ \\
\hline$\nu_{e}, \nu_{\mu}, \nu_{\tau}$ & $g_{V}^{\nu}=\frac{1}{2}$ \\
$e, \mu, \tau$ & $g_{V}^{l}=-\frac{1}{2}+2 \sin ^{2} \theta_{W}$ \\
$u, c, t$ & $g_{V}^{u}=\frac{1}{2}-\frac{4}{3} \sin ^{2} \theta_{w}$ \\
$d, s, b$ & $g_{V}^{d}=-\frac{1}{2}+\frac{2}{3} \sin ^{2} \theta_{w}$ \\
\hline
\end{tabular}

Tabela 3.2: Valores de acoplamento para campos de férmions. $\mathrm{E} \sin ^{2} \theta_{w}=0.231$.

de densidade igual de prótons e elétrons, implicando em cancelamento mútuo do potencial de corrente neutra, restando apenas a contribuição dos nêutrons. Assim,

$$
V_{N C}=-\frac{1}{2} \sqrt{2} G_{F} N_{n}
$$

com $N_{n}$ a densidade de nêutrons e o acoplamento no valor de $-1 / 2$. Então, o potencial efetivo total para baixa temperatura e densidade é a soma das equações $(3.50)$ e (3.53)

$$
V_{\alpha}=V_{C C} \delta_{\alpha e}+V_{N C}=\sqrt{2} G_{F}\left(N_{e} \delta_{\alpha e}-\frac{1}{2} N_{n}\right)
$$

Na tentativa de uma melhor compreensão do significado físico dos potenciais $\left(V_{\alpha}\right)$, vamos compará-los com a energia potencial de um neutrino de sabor qualquer propagando-se no meio. Para neutrinos de Dirac, os potenciais são:

$$
\mathscr{V}_{D \alpha}^{(+)} \simeq V_{\alpha} \frac{m_{\nu_{\alpha}}^{2}}{4}\left\langle\frac{1}{E^{2}}\right\rangle
$$

onde $\mathscr{V}_{D \alpha}^{(+)}$é o potencial para o neutrino de sabor $\alpha$ com helicidade positiva e

$$
\mathscr{V}_{D \alpha}^{(-)} \simeq V_{\alpha}
$$

sendo $\mathscr{V}_{D \alpha}^{(-)}$o equivalente para a helicidade negativa.

Como neutrinos são relativísticos, é de fácil percepção que a Eq. (3.55) é suprimida, uma vez que a massa dos neutrinos é muito pequena. E, pela Eq. (3.56), vemos que a energia potencial é aproximadamente igual ao potencial do neutrino.

No caso dos antineutrinos de Dirac, obtemos resultados opostos: em 
antineutrinos de mão direita a energia potencial se equivale ao potencial dos neutrinos, mas com sinal invertido, e para antineutrinos de mão-esquerda há uma supressão equivalente a da eq. (3.55), também com sinal trocado:

$$
\overline{\mathscr{V}}_{D \alpha}^{(+)} \simeq-V_{\alpha} \quad, \quad \overline{\mathscr{V}}_{D \alpha}^{(-)} \simeq-V_{\alpha} \frac{m_{\nu_{\alpha}}^{2}}{4}\left\langle\frac{1}{E^{2}}\right\rangle
$$

Os resultados para $\mathscr{V}_{D \alpha}^{( \pm)}$e $\overline{\mathscr{V}}_{D \alpha}^{( \pm)}$mostram de maneira clara que, no limite relativístico, os neutrinos podem ser considerados partículas sem massa nas interações e somente os neutrinos de Dirac de mão-esquerda (helicidade negativa) e os antineutrinos de mão-direita (helicidade positiva) participam das interações fracas.

No caso dos neutrinos de Majorana ${ }^{13}$ a energia potencial será

$$
\mathscr{V}_{M \alpha}^{( \pm)} \simeq \mp V_{\alpha}
$$

que coincide com os resultados obtidos para os neutrinos de Dirac. Então, a energia potencial dos neutrinos ultra-relativísticos de Dirac de mão-esquerda e os neutrinos de Majorana são iguais, enquanto que a energia potencial dos antineutrinos relativísticos de Dirac de mão-direita coincide com os neutrinos de Majorana de mão-direita.

\section{5}

\section{Evolução dos Estados de Sabor}

Um neutrino de mão-esquerda, relativístico, com um sabor qualquer $(e, \mu, \tau)$ e momento $\vec{p}$ tem seu estado de sabor descrito pela Eq. (3.1). Mas vimos que os estados de sabor não são auto-estados de massa da Hamiltoniana, então vamos utilizar os neutrinos massivos que são auto-estados da Hamiltoniana no vácuo, $\mathscr{H}_{0}$,

$$
\mathscr{H}_{0}\left|\nu_{k}\right\rangle=E_{k}\left|\nu_{k}\right\rangle
$$

onde $E_{k}$ é fornecido pela Eq. (3.8). O potencial é somado a energia, resultando num Hamiltoniano de dois termos,

$$
\mathscr{H}=\mathscr{H}_{0}+\mathscr{H}_{I}
$$

${ }^{13}$ Nesta classificação os neutrinos são idênticos aos antineutrinos. 
onde $\mathscr{H}_{I}$ é devido ao potencial,

$$
\mathscr{H}_{I}\left|\nu_{\alpha}\right\rangle=V_{\alpha}\left|\nu_{\alpha}\right\rangle
$$

A equação de evolução temporal na representação de Schrödinger é dada pela Eq. (3.9) e, se a multiplicarmos pela esquerda pelo estado $\left\langle\nu_{\beta}\right|$ obtemos a amplitude de transição $\nu_{\alpha} \rightarrow \nu_{\beta}$ após um tempo t,

$$
\psi_{\alpha \beta}(t)=\left\langle\nu_{\beta} \mid \nu_{\alpha}(t)\right\rangle \quad \text { com } \quad \psi_{\alpha \beta}(0)=\delta_{\alpha \beta}
$$

Assim, a Eq. (3.9) terá a forma

$$
i \frac{d}{d t} \psi_{\alpha \beta}(t)=\sum_{\eta}\left(\sum_{k} U_{\beta k} E_{k} U_{\eta k}^{*}+\delta_{\beta \eta} V_{\beta}\right) \psi_{\alpha \eta}(t)
$$

Novamente estamos considerando que neutrinos são relativísticos, ou seja, $p \simeq E$ e $t \simeq x$. Utilizando ainda a equação de conservação de probabilidade $\left(\sum_{\beta} P_{\nu_{\alpha} \rightarrow \nu_{\beta}}(t)=1\right)$ e a Eq. (3.17), reescrevemos a Eq. (3.63) como

$$
\begin{aligned}
i \frac{d}{d x} \psi_{\alpha \beta}(x)= & \left(p+\frac{m_{1}^{2}}{2 E}+V_{N C}\right) \psi_{\alpha \beta}(x) \\
& +\sum_{\eta}\left(\sum_{k} U_{\beta k} \frac{\Delta m_{k 1}^{2}}{2 E} U_{\eta k}^{*}+\delta_{\beta e} \delta_{\eta e} V_{C C}\right) \psi_{\alpha \eta}(x) .
\end{aligned}
$$

Repare que o primeiro termo do lado direito é comum a todas as transições de sabores, o que o torna irrelevante para oscilações pois é eliminado com uma simples mudança de fase. Então, somente o segundo termo da direita é importante para a amplitude de transição. Perceba também que há dependência com a diferença de massa quadrada na oscilação de neutrinos na matéria, do mesmo modo que existe para a oscilação no vácuo. Vamos reescrever a Eq. (3.64), desprezando a fase comum, da seguinte forma:

$$
i \frac{d}{d x} \Psi_{\alpha}=\mathcal{H}_{F} \Psi_{\alpha}
$$

que tem a mesma estrutura da equação de Schrödinger e $\mathcal{H}_{F}$ é a matriz Hamiltoniana efetiva, dada por

$$
\mathcal{H}_{F}=\frac{1}{2 E}\left(U \mathbb{M}^{2} U^{\dagger}+\mathbb{A}\right)
$$


onde para três gerações de neutrinos ativos temos

$$
\Psi_{\alpha}=\left(\begin{array}{c}
\psi_{\alpha e} \\
\psi_{\alpha \mu} \\
\psi_{\alpha \tau}
\end{array}\right), \quad \mathbb{M}^{2}=\left(\begin{array}{ccc}
0 & 0 & 0 \\
0 & \Delta m_{21}^{2} & 0 \\
0 & 0 & \Delta m_{31}^{2}
\end{array}\right), \quad \mathbb{A}=\left(\begin{array}{ccc}
A_{C C} & 0 & 0 \\
0 & 0 & 0 \\
0 & 0 & 0
\end{array}\right)
$$

sendo

$$
A_{C C} \equiv 2 E V_{C C}=2 \sqrt{2} E G_{F} N_{e} .
$$

Um ponto importante é que se estivéssemos tratando o neutrino como partícula de Majorana, isto é, com suas duas fases adicionais, o resultado seria exatamente o mesmo. Observe o primeiro termo do parênteses na Eq. (3.66) e veja que as fases de Majorana se cancelariam. Então, nos experimentos de oscilação de neutrinos, seja no vácuo ou na matéria, não é possível distinguir entre a natureza dos neutrinos (Dirac ou Majorana) [107, 108, 109].

\section{6 \\ Efeito MSW}

Por simplicidade de cálculo, vamos considerar o caso de mistura entre dois neutrinos de sabores $\nu_{e}$ e $\nu_{\mu}$ e os massivos $\nu_{1}$ e $\nu_{2}$, assumindo que no instante inicial $(t=0)$ o neutrino seja eletrônico. Esse seria o caso dos neutrinos produzidos no interior do Sol. Reescrevendo a Eq. (3.67) para duas gerações,

$$
\Psi_{\alpha}=\left(\begin{array}{c}
\psi_{\alpha e} \\
\psi_{\alpha \mu}
\end{array}\right), \quad \mathbb{M}^{2}=\left(\begin{array}{cc}
0 & 0 \\
0 & \Delta m_{21}^{2}
\end{array}\right), \quad \mathbb{A}=\left(\begin{array}{cc}
A_{C C} & 0 \\
0 & 0
\end{array}\right),
$$

a partir de agora vamos fazer $\Delta m_{21}^{2}=\Delta m^{2}$ e como o neutrino é criado com o sabor eletrônico, temos $\alpha=e$. Assim, utilizando as matrizes de (3.69) e a Eq. (3.66), escrevemos a equação de evolução (3.65) como

$$
\begin{aligned}
i \frac{d}{d x}\left(\begin{array}{c}
\psi_{e e} \\
\psi_{e \mu}
\end{array}\right)= & \frac{1}{2 E}\left[\left(\begin{array}{cc}
\cos \theta & \sin \theta \\
-\sin \theta & \cos \theta
\end{array}\right)\left(\begin{array}{cc}
0 & 0 \\
0 & \Delta m^{2}
\end{array}\right)\left(\begin{array}{cc}
\cos \theta & -\sin \theta \\
\sin \theta & \cos \theta
\end{array}\right)+\right. \\
& \left.+\left(\begin{array}{cc}
A_{C C} & 0 \\
0 & 0
\end{array}\right)\right]\left(\begin{array}{l}
\psi_{e e} \\
\psi_{e \mu}
\end{array}\right),
\end{aligned}
$$


e após algumas linhas de desenvolvimento matemático, obtemos

$$
i \frac{d}{d x}\left(\begin{array}{c}
\psi_{e e} \\
\psi_{e \mu}
\end{array}\right)=\frac{1}{4 E}\left(\begin{array}{cc}
-\Delta m^{2} \cos 2 \theta+A_{C C} & \Delta m^{2} \sin 2 \theta \\
\Delta m^{2} \sin 2 \theta & \Delta m^{2} \cos 2 \theta-A_{C C}
\end{array}\right)\left(\begin{array}{l}
\psi_{e e} \\
\psi_{e \mu}
\end{array}\right)
$$

onde desprezamos o termo de fase comum, que é irrelevante.

A probabilidade de transição do neutrino, gerado eletrônico, para o estado de sabor muônico é

$$
P_{\nu_{e} \rightarrow \nu_{\mu}}(x)=\left|\psi_{e \mu}(x)\right|^{2}
$$

e, por consequência, a probabilidade de sobrevivência torna-se

$$
P_{\nu_{e} \rightarrow \nu_{e}}(x)=\left|\psi_{e e}(x)\right|^{2}=1-P_{\nu_{e} \rightarrow \nu_{\mu}}(x)
$$

Podemos diagonalizar a matriz $\mathcal{H}_{F}$ através de uma transformação ortogonal do tipo

$$
U_{M}^{T} \mathcal{H}_{F} U_{M}=\mathcal{H}_{M}
$$

onde $U_{M}$ é a matriz de mistura efetiva na matéria dada por

$$
U_{M}=\left(\begin{array}{cc}
\cos \theta_{M} & \sin \theta_{M} \\
-\sin \theta_{M} & \cos \theta_{M}
\end{array}\right)
$$

e $\mathcal{H}_{M}$ é a Hamiltoniana efetiva na matéria na base auto-estado de massa. Então, usando a eq. (3.75), o cálculo da eq. (3.74) é

$$
\begin{aligned}
\mathcal{H}_{M} & =\frac{1}{4 E}\left(\begin{array}{cc}
\cos \theta_{M} & -\sin \theta_{M} \\
\sin \theta_{M} & \cos \theta_{M}
\end{array}\right)\left(\begin{array}{cc}
-a & b \\
b & a
\end{array}\right)\left(\begin{array}{cc}
\cos \theta_{M} & \sin \theta_{M} \\
-\sin \theta_{M} & \cos \theta_{M}
\end{array}\right) \\
& =\frac{1}{4 E}\left(\begin{array}{cc}
-a \cos 2 \theta_{M}-b \sin 2 \theta_{M} & b \cos 2 \theta_{M}-a \sin 2 \theta_{M} \\
b \cos 2 \theta_{M}-a \sin 2 \theta_{M} & a \cos 2 \theta_{M}+b \sin 2 \theta_{M}
\end{array}\right),
\end{aligned}
$$

onde fizemos $a=\Delta m^{2} \cos 2 \theta-A_{C C}$ e $b=\Delta m^{2} \sin 2 \theta$ por simplicidade de cálculo. Agora, utilizando a relação

$$
\cos 2 \theta_{M}=\frac{\Delta m^{2} \cos 2 \theta-A_{C C}}{\Delta m_{M}^{2}} \quad, \quad \sin 2 \theta_{M}=\frac{\Delta m^{2} \sin 2 \theta}{\Delta m_{M}^{2}},
$$


sendo $\Delta m_{M}^{2}$ a diferença de massa quadrada efetiva, e de onde podemos obter o ângulo de mistura efetiva na matéria:

$$
\tan 2 \theta_{M}=\frac{\tan 2 \theta}{1-\frac{A_{C C}}{\Delta m^{2} \cos 2 \theta}} .
$$

Finalmente chegamos na matriz diagonal de $\mathcal{H}_{F}$ com as relações de (3.77):

$$
\mathcal{H}_{M}=\frac{1}{4 E}\left(\begin{array}{cc}
-\Delta m_{M}^{2} & 0 \\
0 & \Delta m_{M}^{2}
\end{array}\right)
$$

fazendo

$$
\Delta m_{M}^{2}=\sqrt{\left(\Delta m^{2} \cos 2 \theta-A_{C C}\right)^{2}+\left(\Delta m^{2} \sin 2 \theta\right)^{2}} .
$$

Note que temos uma situação de ressonância quando o potencial torna-se

$$
A_{C C}^{R}=\Delta m^{2} \cos 2 \theta \text {. }
$$

Este fato foi observado por Mikheev e Smirnov [110] baseado no trabalho de Wolfenstein [106] entre a segunda metade da década de 70 e a primeira de 80. Por isso, este efeito recebe o nome das iniciais destes três cientistas, ou seja, efeito $M S W$. Pela Eq. (3.78), vemos que na ressonância o ângulo de mistura efetiva é máximo $\left(\theta_{M}=\pi / 4\right)$, deixando a possibilidade de transição entre 2 sabores nesta região maior do que se estivessem no vácuo. Também é neste momento que temos a menor diferença de massa quadrada entre os dois sabores (o primeiro termo da raiz quadrada da Eq. (3.80) desaparece).

\section{7}

\section{Oscilação de Neutrinos em Supernovas}

\subsection{1}

\section{Densidades}

Como vimos no capítulo anterior, uma estrela gigante ou supergigante vermelha possui um raio nuclear da ordem de $10^{4} \mathrm{~km}$ e é nesta região que os neutrinos são criados. No entanto, o raio da estrela pode ultrapassar uma unidade astronômica ${ }^{14}$ (UA) e os neutrinos devem percorrer esta distância para

\footnotetext{
${ }^{14}$ É a distância média da Terra ao Sol e equivale a aproximadamente 150 milhões de km.
} 
alcançar o meio interestelar.

A densidade no interior da pré-supernova é dependente com a sua distância radial, assim como o ângulo de mistura efetivo. Então, as regiões onde podem ocorrer a conversão de um sabor em outro são denominadas camadas de ressonância e a densidade neste local pode ser calculada pela seguinte expressão [111]

$$
\rho_{\text {res }} \sim 1.4 \times 10^{6}\left(\frac{\Delta m^{2}}{1 \mathrm{eV}^{2}}\right)\left(\frac{10 \mathrm{MeV}}{E}\right)\left(\frac{0.5}{Y_{e}}\right) \cos 2 \theta \mathrm{g} / \mathrm{cm}^{3}
$$

o que resulta em duas camadas de ressonância:

1. Alta Densidade $\left(\rho_{H}\right)$ : como o próprio nome diz, a oscilação ocorre em regiões bem povoadas de partículas, tipicamente $10^{3}$ ou $10^{4} \mathrm{~g} / \mathrm{cm}^{3}$ e é comumente associada à diferença de massa quadrada entre $\nu_{2}$ e $\nu_{3}$, isto é, $\Delta m_{a t m}^{2}$.

2. Baixa Densidade $\left(\rho_{L}\right)$ : ocorre em densidades menores e seu valor varia entre 10 e $30 \mathrm{~g} / \mathrm{cm}^{3}$. Esta faixa de densidade é encontrada em regiões intermediárias do Sol e por isso essa densidade é caracterizada pela diferença de massa quadrada entre $\nu_{1}$ e $\nu_{2}$, cujo símbolo é $\Delta m_{\odot}^{2}$.

\subsection{2}

\section{Regiões de Transição}

Podemos reescrever a equação de evolução utilizando os ângulos de mistura efetivos e a diferença de massa quadrada efetiva, mas antes temos de diagonalizar a Hamiltoniana efetiva como

$$
\Psi_{e}=U_{M} \Phi_{e}, \quad \text { sendo } \quad \Psi_{e}=\left(\begin{array}{c}
\psi_{e e} \\
\psi_{e \mu}
\end{array}\right) \text { e } \Phi_{e}=\left(\begin{array}{c}
\phi_{e 1} \\
\phi_{e 2}
\end{array}\right),
$$

assim, a equação de evolução fica

$$
\begin{array}{r}
i \frac{d}{d x}\left(U_{M} \Phi_{e}\right)=\mathcal{H}_{F} U_{M} \Phi_{e} \\
i \Phi_{e} \frac{d U_{M}}{d x}+i U_{M} \frac{d \Phi_{e}}{d x}=\mathcal{H}_{F} U_{M} \Phi_{e}
\end{array}
$$


multiplicando pela esquerda por $U_{M}^{\dagger}$ diagonalizamos a Hamiltoniana e após um exercício algébrico, obtemos

$$
i \frac{d}{d x}\left(\begin{array}{c}
\phi_{e 1} \\
\phi_{e 2}
\end{array}\right)=\frac{1}{4 E}\left(\begin{array}{cc}
-\Delta m_{M}^{2} & -4 E i \frac{d \theta_{m}}{d x} \\
4 E i \frac{d \theta_{m}}{d x} & \Delta m_{M}^{2}
\end{array}\right)\left(\begin{array}{c}
\phi_{e 1} \\
\phi_{e 2}
\end{array}\right) .
$$

Aqui temos 2 opções para trabalhar:

1. Densidade da matéria é constante: neste caso, $\frac{d \theta_{M}}{d x}=0$, o que leva a termos apenas a diagonal principal em (3.85) e a evolução das amplitudes dos neutrinos massivos na matéria é desacoplada. A probabilidade de transição segue os mesmos passos matemáticos que fizemos para a probabilidade de oscilação no vácuo, com exceções do ângulo de mistura e a diferença de massa quadrada que são substituídas por seus equivalentes da matéria (efetivo) e fica

$$
P_{\nu_{e} \rightarrow \nu_{\mu}}(x)=\sin ^{2} 2 \theta_{M} \sin ^{2}\left(\frac{\Delta m_{M}^{2} x}{4 E}\right)
$$

2. Densidade da matéria não é constante: aqui é necessário levar em conta a derivada do ângulo em função da distância. Então, derivando a primeira equação de (3.77) temos

$$
-2 \sin 2 \theta_{M} \frac{d \theta_{M}}{d x}=-\frac{1}{\Delta m_{M}^{2}} \frac{d A_{C C}}{d x}+\frac{\left(\Delta m^{2} \cos 2 \theta-A_{C C}\right)}{\left(\Delta m_{M}^{2}\right)^{2}} \frac{d}{d x} \Delta m_{M}^{2}
$$

e a derivada no último termo é feita utilizando a eq. (3.80) para obtermos

$$
\frac{d \theta_{M}}{d x}=\frac{1}{2} \frac{\sin 2 \theta_{M}}{\Delta m_{M}^{2}} \frac{d A_{C C}}{d x}
$$

A influência da não-constância da densidade na equação de evolução é que ela possiblita transições entre os neutrinos massivos $\left(\nu_{1}^{M}\right.$ e $\left.\nu_{2}^{M}\right)$. Se os termos da diagonal secundária são muito menores que a diferença entre os termos da diagonal principal a probabilidade de transição pode ser desconsiderada devido ao pequeno valor que é obtido.

Para podermos quantificar estas transições vamos introduzir o parâmetro 
de adiabaticidade, que é dado por

$$
\gamma=\frac{\Delta m_{M}^{2}}{4 E\left|d \theta_{M} / d x\right|}=\frac{\left(\Delta m_{M}^{2}\right)^{2}}{2 E \sin 2 \theta_{M}\left|d A_{C C} / d x\right|} \quad .
$$

Ele determina se ocorrerão transições nas camadas da estrela através da Probabilidade de Cruzamento, isto é, a probabilidade de um neutrino com um auto-estado de massa "pular" para outro auto-estado de massa. Esta probabilidade pode ser calculada como [112, 113]

$$
P_{c}=\exp \left(-\frac{\pi}{2} \gamma\right)
$$

Podemos separar as camadas da estrela em três partes distintas:

1. Caso I: se em todos os pontos da trajetória do neutrino temos $\gamma \gg 1$, a evolução será adiabática, ou seja, não haverá transições entre os neutrinos massivos ou estas serão desprezíveis, pois a Probabilidade de Cruzamento (Eq. (3.90)) será muito pequena (Fig. 3.5) e para um neutrino com $E \sim$ $10 \mathrm{MeV}$, o valor do ângulo de mistura $\theta_{13}$ deve obedecer $\sin ^{2} 2 \theta_{13} \gtrsim 10^{-3}$. Este seria o caso mais simples, já que implica na evolução independente das amplitudes e o efeito da evolução seria apenas um fator de fase e teríamos as oscilações de sabores.

2. Caso II: este é o caso quando $\gamma \sim 1$ e $10^{-5} \lesssim \sin ^{2} 2 \theta_{13} \lesssim 10^{-3}$, o que faz com que a Probabilidade de Cruzamento varie fortemente de acordo com a energia do neutrino; quanto maior a energia, maior a chance de cruzamento.

3. Caso III: esta situação é onde o valor do parâmetro de adiabaticidade é muito menor que a unidade e $\sin ^{2} 2 \theta_{13} \lesssim 10^{-5}$, levando a Probabilidade de Cruzamento próxima da unidade $\left(P_{c} \sim 1\right)$, e com grande chance de ocorrer transições entre os neutrinos massivos. Neste caso dizemos que a evolução é não-adiabática.

O ponto de menor valor de $\gamma$ é chamado de máxima violação da adiabaticidade e pode ser calculado a partir da derivada da expressão do $\cos 2 \theta$ da Eq. (3.77) junto com a utilização da Eq. (3.88) para ter a forma

$$
\frac{d A_{C C}}{d x}=-\frac{\Delta m_{M}^{2}}{\sin ^{2} 2 \theta_{M}} \frac{d \cos 2 \theta_{M}}{d x} .
$$


Agora, substituindo na Eq. (3.89), a nova expressão de $\gamma$ é

$$
\gamma=\frac{\Delta m^{2} \sin 2 \theta}{2 E\left|d \cos 2 \theta_{M} / d x\right|}
$$

Para o ponto de máxima violação da adiabaticidade, i.e., quando a primeira derivada é nula, temos

$$
\left.\frac{d^{2} \cos 2 \theta_{M}}{d x^{2}}\right|_{x=x_{\text {max.viol. }}}=0
$$

e das Eqs. (3.77) e (3.81) podemos ver que o ponto de ressonância é dado por

$$
\left.\cos 2 \theta_{M}\right|_{x=x_{R}}=0
$$

Então, em geral, o ponto de mínimo de $\gamma$ não é coincidente com o ponto de ressonância $\left(x_{R}\right)$. Se fizermos a segunda derivada da Eq. (3.77) a Eq. (3.93) pode ser reescrita da forma

$$
\left[3 \cos 2 \theta_{M} \sin 2 \theta_{M}\left(\frac{d A_{C C}}{d x}\right)^{2}+\Delta m^{2} \sin 2 \theta \frac{d^{2} A_{C C}}{d x^{2}}\right]_{x=x_{\text {max.viol. }}}=0
$$

e vemos que se a densidade for linear, os pontos de máxima violação e de ressonância coincidem. Se o ângulo de mistura for muito pequeno, podemos desprezar o segundo termo na Eq. (3.95) e, com boa aproximação, também temos igualdade entre violação e ressonância.

\subsection{3}

\section{Exemplo para Duas Gerações}

A produção de neutrinos no interior das estrelas se dá dentro do núcleo, em regiões de alta densidade, $\rho \rightarrow \infty$, e como os neutrinos muônicos e tauônicos são produzidos e reagem igualmente no interior estelar, podemos reduzir o problema para duas gerações no intuito de uma melhor compreensão. Assim, da estrutura da Eq. (3.83) temos

$$
\begin{aligned}
& \left|\nu_{e}\right\rangle=\cos \theta_{M}(x)\left|\nu_{1}^{M}\right\rangle+\sin \theta_{M}(x)\left|\nu_{2}^{M}\right\rangle \\
& \left|\nu_{\mu}\right\rangle=-\sin \theta_{M}(x)\left|\nu_{1}^{M}\right\rangle+\cos \theta_{M}(x)\left|\nu_{2}^{M}\right\rangle
\end{aligned}
$$




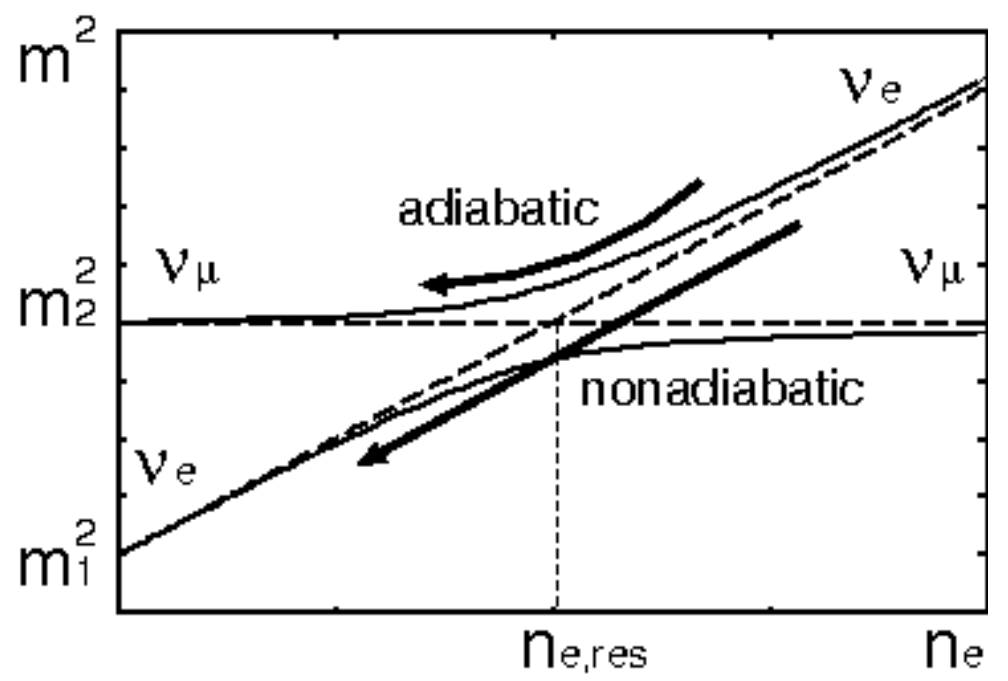

Figura 3.5: Ilustração que mostra a ressonância para dois sabores em um meio inomogêneo. As linhas pontilhadas mostram os auto-estados de massa efetiva quando não há mistura e as linhas contínuas indicam a massa efetiva dos auto-estados de sabor quando existe o efeito de mistura. Note que a menor diferença de massa quadrada efetiva se dá quando a ressonância tem o seu valor mínimo.

onde devido a alta densidade podemos ver o comportamento das funções seno e cosseno dos ângulos de mistura efetivos (Eq. (3.77)) como

$$
\cos 2 \theta_{M}(x) \rightarrow-1 \quad \text { e } \quad \sin 2 \theta_{M}(x) \rightarrow 0 \quad,
$$

o que implica em

$$
\cos \theta_{M}(x) \rightarrow 0 \quad \text { e } \quad \sin \theta_{M}(x) \rightarrow 1 \quad
$$

Nas estrelas, a propagação é feita usando os estados de massa efetiva (ver Eq. 3.85), o que significa que temos de rearrumar as Eqs. (3.96) para que fiquem como

$$
\begin{aligned}
& \left|\nu_{1}^{M}\right\rangle=\cos \theta_{M}(x)\left|\nu_{e}\right\rangle-\sin \theta_{M}(x)\left|\nu_{\mu}\right\rangle \\
& \left|\nu_{2}^{M}\right\rangle=\sin \theta_{M}(x)\left|\nu_{e}\right\rangle+\cos \theta_{M}(x)\left|\nu_{\mu}\right\rangle .
\end{aligned}
$$

Observando as condições expostas acima, vemos que um neutrino eletrônico, quando criado no núcleo estelar, é quase que puramente $\nu_{2}^{M}$ e um neutrino muônico quase $\nu_{1}^{M}$ puro.

Nas camadas mais externas da estrela a densidade torna-se muito menor 
que no local de criação e, por isso, podemos considerá-la como vácuo, $\rho \rightarrow 0$, o que provoca uma mudança nos ângulos de mistura: do valor efetivo para o valor do vácuo,

$$
\cos 2 \theta_{M}(x) \rightarrow \cos 2 \theta \quad \text { e } \quad \sin 2 \theta_{M}(x) \rightarrow \sin 2 \theta
$$

Então, se a propagação for adiabática, isto é, $\gamma \gg 1$, não haverá cruzamento entre os auto-estados de massa $\left(\nu_{1} \nrightarrow \nu_{2}\right)$. Assumindo que o valor do ângulo de mistura no vácuo seja pequeno, temos que as Eqs. (3.99) ficam

$$
\begin{aligned}
& \left|\nu_{\mu}\right\rangle=\left|\nu_{1}^{M}\right\rangle \stackrel{\gamma \gg 1}{\longrightarrow}\left|\nu_{1}^{M}\right\rangle \stackrel{\text { vácuo }}{\longrightarrow}\left|\nu_{1}\right\rangle=\left|\nu_{e}\right\rangle, \\
& \left|\nu_{e}\right\rangle=\left|\nu_{2}^{M}\right\rangle \stackrel{\gamma \gg 1}{\longrightarrow}\left|\nu_{2}^{M}\right\rangle \stackrel{\text { vácuo }}{\longrightarrow}\left|\nu_{2}\right\rangle=\left|\nu_{\mu}\right\rangle,
\end{aligned}
$$

isto é, o neutrino criado muônico (eletrônico) possui auto-estado de massa efetiva $\nu_{1}^{M}\left(\nu_{2}^{M}\right)$ e durante o seu caminho para fora da estrela passa de uma região de alta densidade para outra de baixa densidade, ou vácuo, mas o autoestado de massa continua o mesmo, mudando apenas o seu valor (no vácuo, a estrutura é análoga a Eq. (3.99) apenas com a mudança de valores dos ângulos de mistura) e o neutrino resultante tem o sabor eletrônico (muônico) como pode ser observado na Fig. 3.5.

Agora, se $\gamma \ll 1$ ocorre a transição entre os auto-estados de massa $\left(\nu_{1}^{M} \rightarrow \nu_{2}^{M}\right)$ na passagem do neutrino pela região de ressonância, ou seja, o esquema de (3.101) fica

$$
\begin{gathered}
\left|\nu_{\mu}\right\rangle=\left|\nu_{1}^{M}\right\rangle \stackrel{\gamma \ll 1}{\longrightarrow}\left|\nu_{2}^{M}\right\rangle \stackrel{\text { vácuo }}{\longrightarrow}\left|\nu_{2}\right\rangle=\left|\nu_{\mu}\right\rangle, \\
\left|\nu_{e}\right\rangle=\left|\nu_{2}^{M}\right\rangle \stackrel{\gamma \ll 1}{\longrightarrow}\left|\nu_{1}^{M}\right\rangle \stackrel{\text { vácuo }}{\longrightarrow}\left|\nu_{1}\right\rangle=\left|\nu_{e}\right\rangle .
\end{gathered}
$$

Se considerarmos antineutrinos, as Eqs. (3.96) não sofrem alterações, mas como o potencial é negativo para os antineutrinos, a Eq. (3.97) passa a ter a forma

$$
\cos 2 \theta_{M}(x) \rightarrow 1 \quad \text { e } \quad \sin 2 \theta_{M}(x) \rightarrow 0 \quad,
$$

implicando em $\theta_{M}=0$. Observando as Eqs. (3.99), mas utilizando o novo valor do ângulo de mistura efetiva, vemos que o antineutrino eletrônico é criado como $\bar{\nu}_{1}^{M}$ e o antineutrino muônico como $\bar{\nu}_{2}^{M}$. Quando a densidade torna-se menor $(\rho \rightarrow 0)$ há a mudança nos ângulos de mistura que passam a ter os valores 
equivalentes ao vácuo, como explicamos para o caso dos neutrinos. Assim, se a propagação for adiabática, isto é, $\gamma \gg 1$, o equivalente às Eqs. (3.101) é

$$
\begin{aligned}
& \left|\bar{\nu}_{e}\right\rangle=\left|\bar{\nu}_{1}^{M}\right\rangle \stackrel{\gamma \gg 1}{\longrightarrow}\left|\bar{\nu}_{1}^{M}\right\rangle \stackrel{\text { vácuo }}{\longrightarrow}\left|\bar{\nu}_{1}\right\rangle=\left|\bar{\nu}_{e}\right\rangle, \\
& \left|\bar{\nu}_{\mu}\right\rangle=\left|\bar{\nu}_{2}^{M}\right\rangle \stackrel{\gamma \gg 1}{\longrightarrow}\left|\bar{\nu}_{2}^{M}\right\rangle \stackrel{\text { vácuo }}{\longrightarrow}\left|\bar{\nu}_{2}\right\rangle=\left|\bar{\nu}_{\mu}\right\rangle,
\end{aligned}
$$

onde podemos perceber que o antineutrino eletrônico (muônico), criado como $\bar{\nu}_{1}^{M}\left(\bar{\nu}_{2}^{M}\right)$ não muda de sabor em seu trajeto de fuga da estrela, isto é, não oscila.

Para o caso em que $\gamma \ll 1$, o resultado será o mesmo, tendo em vista que não haverá o cruzamento entre os auto-estados dos antineutrinos massivos, pois não existe ponto de ressonância no canal dos antineutrinos.

Concluindo, podemos dizer que a densidade pela qual o neutrino passa no seu trajeto em direção ao espaço é importante para ocorrer ou não a oscilação de sabor, onde para um índice de adiabaticidade alto temos oscilação enquanto que para um valor baixo de gama $(\gamma)$ as oscilações são fortemente suprimidas. Por outro lado, por não haver ponto de ressonância que afete a propagação dos antineutrinos, estes percorrem o caminho sem sofrer oscilações.

\section{8}

\section{Probabilidade de Oscilação e Fluxo de Neutrinos em Detectores}

A Fig. 3.6 [111] nos mostra a evolução dos neutrinos e antineutrinos desde o local de onde são criados, no interior da pré-SN, em alta densidade, até escaparem da estrela, alcançando o espaço (vácuo - próximo do zero da abscissa), tanto para a hierarquia normal (a) quanto para a hierarquia invertida (b). Também podemos notar as duas camadas de ressonância: alta $(\mathrm{H})$ para neutrinos atmosféricos e baixa (L) para os neutrinos solares e que situam-se em posições diferentes, dependendo da hierarquia adotada. No caso da hierarquia normal (à esquerda) as camadas de ressonância afetam apenas a propagação dos neutrinos, enquanto que para a hierarquia invertida (à direita) a camada de alta ressonância influencia a propagação dos antineutrinos e não afeta os neutrinos.

Subdividimos esta seção em duas partes: a primeira, considerando hierarquia normal e a segunda, para hierarquia invertida, sendo que dentro de cada parte novamente subdividimos o estudo para o fluxo dos neutrinos, antineutrinos e o efeito da conversão. 
(a)

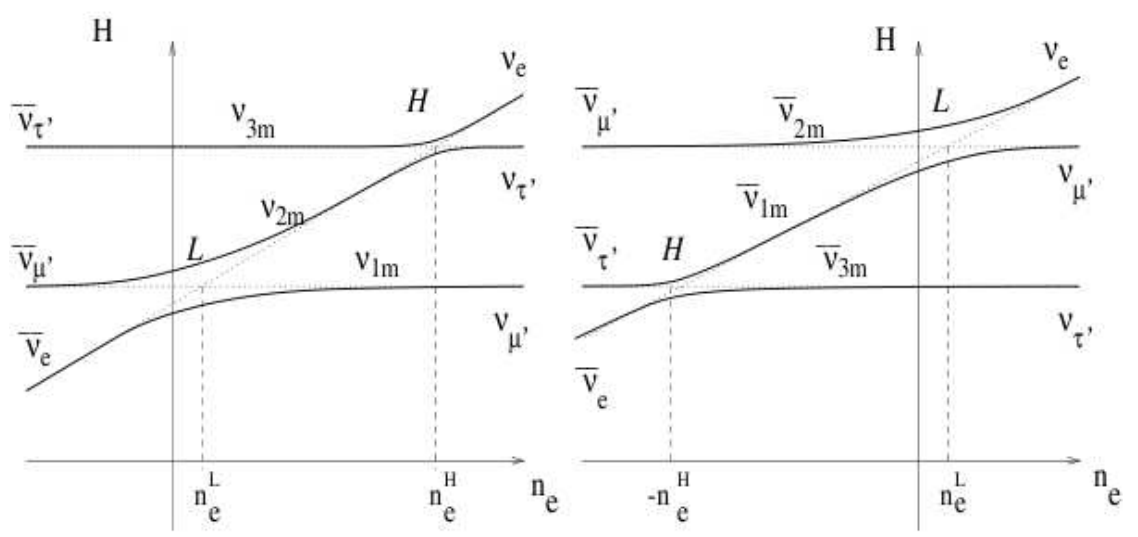

Figura 3.6: Diagramas dos níveis de cruzamento para as hierarquias a) normal e b) invertida. As linhas sólidas mostram a evolução da Hamiltoniana efetiva como função da densidade eletrônica enquanto que as linhas pontilhadas correspondem aos sabores. Densidade negativa é referente ao caso dos antineutrinos.

\subsection{1}

\section{Hierarquia Normal}

\section{Neutrinos}

Os neutrinos são produzidos no interior estelar, em regiões de alta densidade, $\rho \rightarrow \infty$ (ou $\rho \gg \rho_{H}, \rho_{L}$ ), o que leva a uma forte supressão das misturas. Observando a Fig. 3.6a vemos que eles são criados como,

$$
\nu_{e}=\nu_{3}^{M} \quad, \quad \nu_{\mu}=\nu_{1}^{M} \quad, \quad \nu_{\tau}=\nu_{2}^{M} .
$$

Também vamos considerar os fluxos dos auto-estados neste local como originais e da forma

$$
F_{1}^{0(M)}=F_{x}^{0} \quad, \quad F_{2}^{0(M)}=F_{x}^{0} \quad, \quad F_{3}^{0(M)}=F_{e}^{0},
$$

onde denotamos pelo subíndice $\mathrm{x}$ os neutrinos tauônicos e muônicos, tendo em vista que eles reagem da mesma forma (corrente neutra) e são gerados em quantidades iguais.

As duas camadas de ressonância que vimos na subseção 3.7.1 são independentes no que diz respeito à dinâmica de transição. Então podemos denominar de $P_{H}$ e $P_{L}$ as probabilidades de cruzamento dos auto-estados massivos nas regiões de alta e baixa densidade, respectivamente. 
Vamos analisar primeiramente o fluxo do auto-estado $\nu_{1}$ ao chegar na superfície da estrela. $\mathrm{O}$ neutrino originado eletrônico $\left(\nu_{3}^{M}\right)$ tem uma probabilidade $P_{H} P_{L}$ de chegar à superfície como $\nu_{1}$, pois houve o cruzamento $\nu_{3}^{M} \rightarrow \nu_{2}^{M}$ e posteriormente a troca $\nu_{2}^{M} \rightarrow \nu_{1}^{M}$ antes dele sair para o espaço. Assim, a contribuição dos neutrinos eletrônicos originais pode ser expressa

$$
F_{1}=P_{H} P_{L} F_{e}^{0}
$$

Os neutrinos muônicos no centro da estrela $\left(\nu_{1}^{M}\right)$ que oscilaram para o tipo eletrônico podem ser encontrados da seguinte maneira: sabemos que a quantidade de neutrinos massivos que mudam seu auto-estado é dado pela probabilidade $P_{L}$, então o número de neutrinos massivos que permaneceu no mesmo auto-estado de massa é $\left(1-P_{L}\right)$. Desse modo,

$$
F_{1}=\left(1-P_{L}\right) F_{x}^{0}
$$

e para o neutrino criado com sabor tauônico seguimos a mesma lógica do muônico usada acima para obtermos

$$
F_{1}=P_{L}\left(1-P_{H}\right) F_{x}^{0}
$$

O fluxo total de neutrinos que chegam à superfície da pré-supernova é encontrado ao somarmos as Eqs. (3.107 - 3.109)

$$
\begin{aligned}
F_{1} & =P_{H} P_{L} F_{e}^{0}+\left(1-P_{L}\right) F_{x}^{0}+P_{L}\left(1-P_{H}\right) F_{x}^{0} \\
& =P_{H} P_{L} F_{e}^{0}+\left(1-P_{H} P_{L}\right) F_{x}^{0} .
\end{aligned}
$$

Usando o mesmo raciocínio para os outros 2 auto-estados de massa, obtemos

$$
\begin{aligned}
F_{2} & =P_{H}\left(1-P_{L}\right) F_{e}^{0}+P_{L} F_{x}^{0}+\left(1-P_{H}\right)\left(1-P_{L}\right) F_{x}^{0} \\
& =P_{H}\left(1-P_{L}\right) F_{e}^{0}+\left(1-P_{H}+P_{H} P_{L}\right) F_{x}^{0}, \\
F_{3} & =\left(1-P_{H}\right) F_{e}^{0}+P_{H} F_{x}^{0}+0 F_{x}^{0} \\
& =\left(1-P_{H}\right) F_{e}^{0}+P_{H} F_{x}^{0} .
\end{aligned}
$$

Como os detectores utilizados atualmente tem capacidade de captar apenas os sabores, não os auto-estados de massa, precisamos reescrever as Eqs. (3.110) e 
(3.111a) como

$$
F_{i}=a_{i} F_{e}^{0}+\left(1-a_{i}\right) F_{x}^{0}
$$

onde

$$
a_{1}=P_{H} P_{L}, \quad a_{2}=P_{H}\left(1-P_{L}\right), \quad a_{3}=1-P_{H}
$$

Levando em conta a condição de unitariedade da matriz de mistura e usando a Eq. (3.112) podemos encontrar o fluxo dos neutrinos eletrônicos que chegam à Terra como

$$
\begin{aligned}
F_{e} & =\sum_{i}\left|U_{e i}\right|^{2} F_{i} \\
& =\sum_{i}\left|U_{e i}\right|^{2} a_{i} F_{e}^{0}+\left(1-\sum_{i}\left|U_{e i}\right|^{2} a_{i}\right) F_{x}^{0}
\end{aligned}
$$

e é possível, novamente, modificar a expressão (3.114) para

$$
F_{e}=p F_{e}^{0}+(1-p) F_{x}^{0}
$$

sendo que

$$
p \equiv \sum_{i}\left|U_{e i}\right|^{2} a_{i}
$$

pode ser compreendido como a probabilidade de sobrevivência total do neutrino eletrônico.

Sabendo que o fluxo total de neutrinos das três espécies permanece constante, podemos determinar os fluxos de $\nu_{\mu}$ e $\nu_{\tau}$ que chegam ao planeta como

$$
\begin{aligned}
F_{e}^{0}+2 F_{x}^{0}=F_{e}+F_{\mu}+F_{\tau} & \\
F_{\mu}+F_{\tau} & =F_{e}^{0}+2 F_{x}^{0}-F_{e} \\
& =(1-p) F_{e}^{0}+(1+p) F_{x}^{0} .
\end{aligned}
$$

Chamamos a atenção para o fato da divergência dos pacotes de onda, isto é, qualquer coerência entre os auto-estados de massa é desfeita no trajeto para a Terra. Isto significa que os fluxos que chegam aqui não possuem qualquer ligação entre os auto-estados de massa [111]. 


\section{Antineutrinos}

Em densidades muito altas $\left(\rho \gg \rho_{H}, \rho_{L}\right)$ a mistura dos auto-estados de massa é fortemente suprimida, semelhante ao caso dos neutrinos. Então, observando novamente a Fig. 3.6a, mas agora pelo lado esquerdo, vemos que a produção dos antineutrinos se dá como

$$
\bar{\nu}_{e}=\bar{\nu}_{1}^{M} \quad, \quad \bar{\nu}_{\mu}=\bar{\nu}_{2}^{M} \quad, \quad \bar{\nu}_{\tau}=\bar{\nu}_{3}^{M},
$$

e os fluxos iniciais serão

$$
\bar{F}_{1}^{0(M)}=\bar{F}_{e}^{0} \quad, \quad \bar{F}_{2}^{0(M)}=\bar{F}_{x}^{0} \quad, \quad \bar{F}_{3}^{0(M)}=\bar{F}_{x}^{0} .
$$

Há uma forte supressão na matéria sobre o já diminuto ângulo $\theta_{13}$, fazendo com que a transição $\bar{\nu}_{1} \leftrightarrow \bar{\nu}_{3}$ seja desprezível (veja Fig. 3.6). Então o $\bar{\nu}_{3}^{M}$ propaga-se adiabaticamente, de modo que

$$
\bar{\nu}_{\tau}=\bar{\nu}_{3}^{M} \rightarrow \bar{\nu}_{3}=\bar{\nu}_{\tau}
$$

Do mesmo modo que fizemos para o caso dos neutrinos, vamos chamar de $\bar{P}_{H}$ e $\bar{P}_{L}$ as probabilidades de cruzamento dos auto-estados massivos dos antineutrinos em altas e baixas densidades, respectivamente. Também podemos utilizar o mesmo raciocínio para montarmos a expressão dos fluxos

$$
\bar{F}_{e}=\bar{p} \bar{F}_{e}^{0}+(1-\bar{p}) \bar{F}_{x}^{0},
$$

e

$$
\bar{F}_{\mu}+\bar{F}_{\tau}=(1-\bar{p}) \bar{F}_{e}^{0}+(1+\bar{p}) \bar{F}_{x}^{0}
$$

sendo $\bar{p}$ novamente a probabilidade de sobrevivência, agora para os antineutrinos. Sua forma é igual a da Eq. (3.116),

$$
\begin{aligned}
\bar{p} & \equiv \sum_{i}\left|U_{e i}\right|^{2} a_{i} \\
& =\left|U_{e 1}\right|^{2}\left(1-\bar{P}_{L}\right)+\left|U_{e 2}\right|^{2} \bar{P}_{L} .
\end{aligned}
$$

Como já mencionamos anteriormente, os neutrinos do múon e do tau reagem do mesmo modo, via corrente neutra. O mesmo vale para suas anti- 
partículas. Então, daqui em diante, vamos denotá-los apenas por $F_{x}$, sem a necessidade de distinguí-los. Agora vamos levar em consideração os casos de conversão.

\section{Efeitos da Conversão}

Os neutrinos produzidos pelo Sol, chamados de neutrinos solares, representam a conversão ressonante " $\nu_{e} \rightarrow \nu_{2}$ " que ocorre no interior da nossa estrela. Observando o lado esquerdo da Fig. 3.6a podemos ver que os antineutrinos não passam por nenhuma camada de ressonância, embora haja alguma transição $\bar{\nu}_{1} \leftrightarrow \bar{\nu}_{2}$ o que significa que a propagação se dá adiabaticamente $\left(\bar{P}_{L} \simeq 0\right)$. Da Eq. (3.123) podemos determinar a probabilidade de sobrevivência dos antineutrinos eletrônicos

$$
\bar{p} \simeq\left|U_{e 1}\right|^{2} \simeq \cos ^{2} \theta_{\odot},
$$

onde, na última igualdade, fizemos $\cos \theta_{13} \simeq 1$. Podemos obter o valor numérico para o resultado mostrado na Eq. (3.124) ao lembrarmos que $\sin ^{2} \theta_{\odot} \sim 0.31$ [57].

Considerando os valores atuais de $\Delta m_{\odot}^{2}$ e $\sin ^{2} 2 \theta_{\odot}$ [57], a probabilidade de sobrevivência dos neutrinos eletrônicos em baixas densidades é aproximadamente zero $\left(P_{L} \simeq 0\right)$, conforme podemos ver na Fig. 4 da Ref. [111]. Das Eqs. (3.116) e (3.113) temos

$$
\begin{aligned}
p & \simeq\left|U_{e 2}\right|^{2} P_{H}+\left|U_{e 3}\right|^{2}\left(1-P_{H}\right) \\
& =\left(\sin \theta_{e 2} \cos \theta_{e 3}\right)^{2} P_{H}+\left(\sin \theta_{e 3}\right)^{2}\left(1-P_{H}\right) \\
& =\sin ^{2} \theta_{\odot} P_{H}+\left|U_{e 3}\right|^{2}\left(1-P_{H}\right) .
\end{aligned}
$$

De acordo com os recentes resultados dos experimentos Daya Bay [114] e RENO [115] na observação do desaparecimento de antineutrinos eletrônicos de reatores, o valor de $\theta_{13}$ se enquadra na situação onde $\gamma \gg 1$, i.e., a evolução adiabática. Assim, temos que $P_{H} \simeq 0$ e a Eq. (3.125) fica

$$
p=\left|U_{e 3}\right|^{2} \quad,
$$


e o espectro que podemos esperar na Terra é

$$
\begin{aligned}
F_{e} & \approx\left|U_{e 3}\right|^{2} F_{e}^{0}+\left(1-\left|U_{e 3}\right|^{2}\right) F_{x}^{0} \approx F_{x}^{0}, \\
\bar{F}_{e} & \approx \cos ^{2} \theta_{\odot} \bar{F}_{e}^{0}+\sin ^{2} \theta_{\odot} F_{x}^{0}, \\
4 F_{x} & \approx F_{e}^{0}+\sin ^{2} \theta_{\odot} \bar{F}_{e}^{0}+\left(2+\cos ^{2} \theta_{\odot}\right) F_{x}^{0} .
\end{aligned}
$$

Destacamos alguns fatos relevantes: o canal $\nu_{e}$ é suprimido pelo ângulo de $\theta_{13}$, aparecendo no canal $\nu_{x}$; o espectro de $\bar{\nu}_{e}$ é uma mistura de seus originais $\left(\bar{\nu}_{e}\right)$ e do espectro de $\nu_{x}$; e o espectro de $\nu_{x}$ contém partes de todos os espectros originados no núcleo da estrela.

\subsection{2}

\section{Hierarquia Invertida}

Agora vamos considerar que o auto-estado de massa mais leve é o $\nu_{3}$ e os auto-estados $\nu_{1}$ e $\nu_{2}$ são muito mais pesados e degenerados. Na Fig. 3.6b vemos que temos a camada de alta ressonância $\left(\rho_{H}\right)$ no setor dos antineutrinos e a de baixa ressonância $\left(\rho_{L}\right)$ no de neutrinos.

\section{Neutrinos}

O local onde os neutrinos são gerados é de densidade muito alta $(\rho \gg$ $\left.\rho_{H}, \rho_{L}\right)$ o que suprime a mistura dos auto-estados de massa. Neste ponto temos

$$
\nu_{e}=\nu_{2}^{M} \quad, \quad \nu_{\mu}=\nu_{1}^{M} \quad, \quad \nu_{\tau}=\nu_{3}^{M}
$$

O neutrino eletrônico gerado no núcleo chega na camada de ressonância para baixas densidades ainda com seu auto-estado de massa em $\nu_{2}^{M}$, pois a camada de ressonância para altas densidades está no canal dos antineutrinos. Então, a probabilidade do auto-estado $\nu_{2}^{M}$ passar para $\nu_{1}^{M}$ será $P_{L}$ e de permanecer como $\nu_{2}^{M}$ será de $\left(1-P_{L}\right)$. Os fluxos originais podem ser descritos como

$$
F_{1}^{0(M)}=F_{x}^{0} \quad, \quad F_{2}^{0(M)}=F_{e}^{0} \quad, \quad F_{3}^{0(M)}=F_{x}^{0},
$$


e os fluxos totais dos neutrinos na superfície da estrela são

$$
\begin{aligned}
& F_{1}=P_{L} F_{e}^{0}+\left(1-P_{L}\right) F_{x}^{0}, \\
& F_{2}=\left(1-P_{L}\right) F_{e}^{0}+P_{L} F_{x}^{0}, \\
& F_{3}=F_{3}^{0} .
\end{aligned}
$$

Usando a Eq. (3.112) temos que

$$
a_{1}=P_{L} \quad \text { e } \quad a_{2}=\left(1-P_{L}\right),
$$

que nos leva à probabilidade de sobrevivência (p) do neutrino eletrônico na forma

$$
\begin{aligned}
p & \equiv \sum_{i}\left|U_{e i}\right|^{2} a_{i} \\
& =\left|U_{e 1}\right|^{2} P_{L}+\left|U_{e 2}\right|^{2}\left(1-P_{L}\right) .
\end{aligned}
$$

\section{Antineutrinos}

Para os antineutrinos temos que no local de criação os seus auto-estados são relacionados com os sabores como

$$
\bar{\nu}_{e}=\bar{\nu}_{3}^{M} \quad, \quad \bar{\nu}_{\mu}=\bar{\nu}_{2}^{M} \quad, \quad \bar{\nu}_{\tau}=\bar{\nu}_{1}^{M},
$$

de acordo com a Fig. 3.6b, e a relação dos fluxos iniciais é

$$
\bar{F}_{1}^{0(M)}=F_{x}^{0} \quad, \quad \bar{F}_{2}^{0(M)}=F_{x}^{0} \quad, \quad \bar{F}_{3}^{0(M)}=\bar{F}_{e}^{0} .
$$

Seguindo os mesmos passos utilizados para os neutrinos, podemos encontrar a probabilidade de sobrevivência do antineutrino eletrônico como

$$
\bar{p}=\left|U_{e 1}\right|^{2} \bar{P}_{H}\left(1-\bar{P}_{L}\right)+\left|U_{e 2}\right|^{2} \bar{P}_{H} \bar{P}_{L}+\left|U_{e 3}\right|^{2}\left(1-\bar{P}_{H}\right)
$$

\section{Efeitos da Conversão}

As transições dos neutrinos na camada de baixa densidade $\left(\rho_{L}\right)$ são adiabáticas $\left(P_{L} \simeq 0\right)$. Então a probabilidade de sobrevivência do neutrino 
eletrônico representado na Eq. (3.134) fica

$$
p \approx\left|U_{e 2}\right|^{2} \approx \sin ^{2} \theta_{\odot}
$$

e o espectro fica

$$
F_{e} \approx \sin ^{2} \theta_{\odot} F_{e}^{0}+\cos ^{2} \theta_{\odot} F_{x}^{0}
$$

As transições dos antineutrinos em baixa densidade também se dão adiabaticamente, fazendo $\bar{P}_{L} \simeq 0$. Na camada de alta densidade, $\rho_{H}$, o espectro do $\bar{\nu}_{e}$ depende da localização de $\left|U_{e 3}\right|^{2}$.

Então, analogamente ao caso da hierarquia normal, o valor de $\theta_{13}>10^{-3}$ implica em evolução adiabática $(\gamma \gg 1), \log o \bar{P}_{H} \simeq 0$. Desse modo podemos escrever a Eq. (3.137) na forma $\bar{p} \simeq\left|U_{e 3}\right|^{2}$ e como o valor de $\left|U_{e 3}\right|^{2}$ é muito pequeno podemos ver que o espectro do $\bar{\nu}_{e}$ é praticamente o espectro original do $\nu_{x}$, que pode ser escrito como

$$
\bar{F}_{e} \simeq F_{x}^{0}
$$

Uma característica interessante é o fato de que os espectros dos neutrinos são compostos, isto é, misturados pelas transições, mas o espectro dos antineutrinos eletrônicos é praticamente o original do $\nu_{x}$.

\section{9}

\section{Quantificando o Efeito de Matéria}

Devido aos recentes resultados de medida de $\theta_{13}$ mostrarem que o valor de $\sin ^{2} 2 \theta_{13} \simeq 0.1[114,115,116]$, o efeito de matéria da Terra só é efetivo para a hierarquia normal de massa e, nessa situação, os efeitos coletivos, ondas de choque entre outros são muito pequenos para $\bar{\nu}_{e}$.

Embora o impacto de $\theta_{13} \neq 0$ seja maior para a hierarquia invertida, i.e., afeta $\bar{p}(E)$ de modo mais significativo, o efeito de matéria da Terra não é grande.

Assim podemos escrever o fluxo de $\bar{\nu}_{e}$ que passaram pelo interior da Terra como

$$
F_{\bar{\nu}_{e}}^{\oplus}(E)=\bar{p}^{\oplus}(E) F_{\bar{\nu}_{e}}^{0}(E)+\left[1-\bar{p}^{\oplus}(E)\right] F_{\bar{\nu}_{x}}^{0}(E),
$$


onde

$$
\bar{p}^{\oplus}(E)=\frac{1}{\left|U_{e 2}\right|^{2}-\left|U_{e 1}\right|^{2}}\left[\left\{\left|U_{e 2}\right|^{2}-\bar{p}(E)\right\} \bar{p}_{1 e}^{\oplus}+\left\{\bar{p}(E)-\left|U_{e 1}\right|^{2}\right\} \bar{p}_{2 e}^{\oplus}\right],
$$

e $U_{e k}(k=1,2)$ são os elementos da matriz de mistura (Eq. 3.3) que relaciona os autoestados de massa e sabor. A probabilidade de um autoestado de massa penetrar na Terra como $\bar{\nu}_{k}$ e ser detectado, ao sair, como $\bar{\nu}_{e}$, após viajar uma distância $L$ no interior da Terra, é dado por

$$
\bar{p}_{k e}^{\oplus} \equiv P^{\oplus}\left(\bar{\nu}_{k} \rightarrow \bar{\nu}_{e}, L\right), \quad(k=1,2) .
$$

Agora podemos fazer a diferença entre os fluxos de neutrinos observados, i.e., os espectros com e sem efeito de matéria da Terra utilizando as Eqs. (3.121) e (3.141),

$$
\begin{aligned}
\Delta F_{\bar{\nu}_{e}} \equiv & F_{\bar{\nu}_{e}}^{\oplus}(E)-F_{\bar{\nu}_{e}}(E) \\
= & \frac{1}{\left|U_{e 1}\right|^{2}-\left|U_{e 2}\right|^{2}}\left\{F_{\bar{\nu}_{e}}^{0}(E)-F_{\bar{\nu}_{x}}^{0}(E)\right\} \times \\
& \left\{[2 \bar{p}(E)-1]\left(\left|U_{e 2}\right|^{2}-\bar{p}_{2 e}^{\oplus}\right)+\left|U_{e 3}\right|^{2}\left(\bar{p}(E)-\bar{p}_{2 e}^{\oplus}\right)\right\} \\
\simeq & \frac{1}{\cos 2 \theta_{12}}[2 \bar{p}(E)-1]\left(s_{12}^{2}-\bar{p}_{2 e}^{\oplus}\right)\left\{F_{\bar{\nu}_{e}}^{0}(E)-F_{\bar{\nu}_{x}}^{0}(E)\right\} \\
\simeq & \left(\bar{p}_{1 e}^{\oplus}-c_{12}^{2}\right)\left\{F_{\bar{\nu}_{e}}^{0}(E)-F_{\bar{\nu}_{x}}^{0}(E)\right\},
\end{aligned}
$$

onde $\bar{p}(E) \simeq c_{13}^{2}$ foi utilizado para obtermos a expressão final e o termo proporcional a $\left|U_{e 3}\right|^{2}$ pode ser descartado porque contribui apenas com $7 \%$ do primeiro termo. Para observar o efeito de matéria da Terra, Eq. (3.144), a diferença entre os espectros na origem de $F_{\bar{\nu}_{e}}^{0}(E)$ e $F_{\bar{\nu}_{x}}^{0}(E)$, precisa ser significativamente grande e o desvio de $\bar{p}_{1 e}^{\oplus}$ de $c_{12}^{2}$ também precisa ser suficiente grande. 


\section{4}

\section{Detector de Neutrinos no ANDES}

\section{1}

\section{Laboratórios Subterrâneos}

Os primeiros experimentos subterrâneos na física de neutrinos ocorreram na década de 60, para a detecção de neutrinos atmosféricos - produzidos por raios cósmicos ao interagirem com a atmosfera terrestre - e solares. Para os atmosféricos destacamos os experimentos realizados em minas de ouro: 1) na Índia [117], mina Kolar, a 2700 m de profundidade e, 2) na África do Sul [118], mina East Rand, a 3200 m abaixo do solo. Para detecção de neutrinos solares, R. Davis utilizou a mina de Homestake [119], no EUA.

A partir de então, os experimentos subterrâneos deixaram de ser apenas um "compartimento", para se tornarem verdadeiros laboratórios, com estrutura completa: circulação de ar, circulação de água, controle de temperatura e umidade, além de atender a requisitos de segurança em caso de sinistros e apoio na superfície. Neste sentido, o primeiro laboratório subterrâneo construído foi o Baksan Neutrino Observatory na região de Baksan, na extinta União Soviética.

No final da década de 70, a construção de um túnel duplo, sob a montanha de Gran Sasso, na Itália, para ligar Roma ao mar Adriático, fez com que o Instituto Nazionale di Fisica Nucleare sugerisse a construção de um complexo subterrâneo que abrangesse vários campos de pesquisa, inclusive neutrinos. A sugestão foi aprovada pelo governo italiano e no final da década de $80 \mathrm{o}$ laboratório foi entregue para a comunidade científica. Seu custo foi considerado baixo em comparação ao gasto total da obra.

Seguindo a tendência, o Japão utilizou o espaço da antiga mina de Kamioka para construir um detector de água Cherenkov na década de 80, abrangendo uma ampla gama de projetos. Com o sucesso do experimento, o detector foi ampliado na década seguinte para o Super-Kamiokande, que detectou o fenômeno de oscilação de neutrinos, em 1998. 
Inúmeros outros detectores de variadas dimensões foram construídos a partir de 1980 atendendo a comunidade científica de maneira bastante diversificada.

A necessidade de construirmos laboratórios a grandes profundidades varia de acordo com o experimento a ser feito, que difere muito de um caso para outro. O estudo de neutrinos de supernovas, geoneutrinos, ondas gravitacionais, ondas sísmicas, movimentos de placas e vida sob condições extremas são alguns exemplos de pesquisas realizadas centenas de metros abaixo da superfície. No caso do estudo de neutrinos de supernovas, por exemplo, o principal motivo é a diminuição de ruído devido a múons atmosféricos, criados na interação de raios cósmicos com a atmosfera.

As camadas de rocha que separam um laboratório subterrâneo da superfície agem como uma parede, diminuindo quase que exponencialmente o fluxo de múons com a profundidade. Como existem vários tipos de rochas, normalmente calcula-se o nível da blindagem em metros - ou quilômetros - de água-equivalente. Isto é, calculamos como se o detector estivesse no oceano e a água do mar agisse como protetor. Em primeira aproximação, podemos equiparar $300 \mathrm{~m}$ de rocha a $1 \mathrm{~km}$ de água-equivalente.

Na Fig. 4.1, retirada da Ref. [120], podemos observar o decaimento do fluxo de múons atmosféricos em função da blindagem proporcionada pelas rochas. Destaque para as minas de Kolar e East Rand, na Índia e África do Sul, respectivamente, que são muito profundas e possuem um fluxo muito baixo de múons e foram utilizadas na década de 60 para detecção de neutrinos atmosféricos. Dos laboratórios atuais, o de Sudbury é o que possui a melhor blindagem, $\sim 6000 \mathrm{~m}$ de água-equivalente.

Agora vamos fazer uma rápida análise dos laboratórios mais importantes atualmente mencioando suas principais características. A saber: Gran Sasso, Kamioka e SNOLAB.

\subsection{1}

\section{Gran Sasso}

O Laboratório Nacional de Gran Sasso (LNGS) é o maior laboratório subterrâneo do mundo em experimentos de física de partículas, astrofísica de partículas e astrofísica nuclear, envolvendo mais de mil cientistas de $\sim 30$ países todo ano. A Fig. 4.2 mostra o complexo do LNGS onde hoje há $\sim 15$ experimentos em atividade que necessitam de ambiente com baixo ruído. 


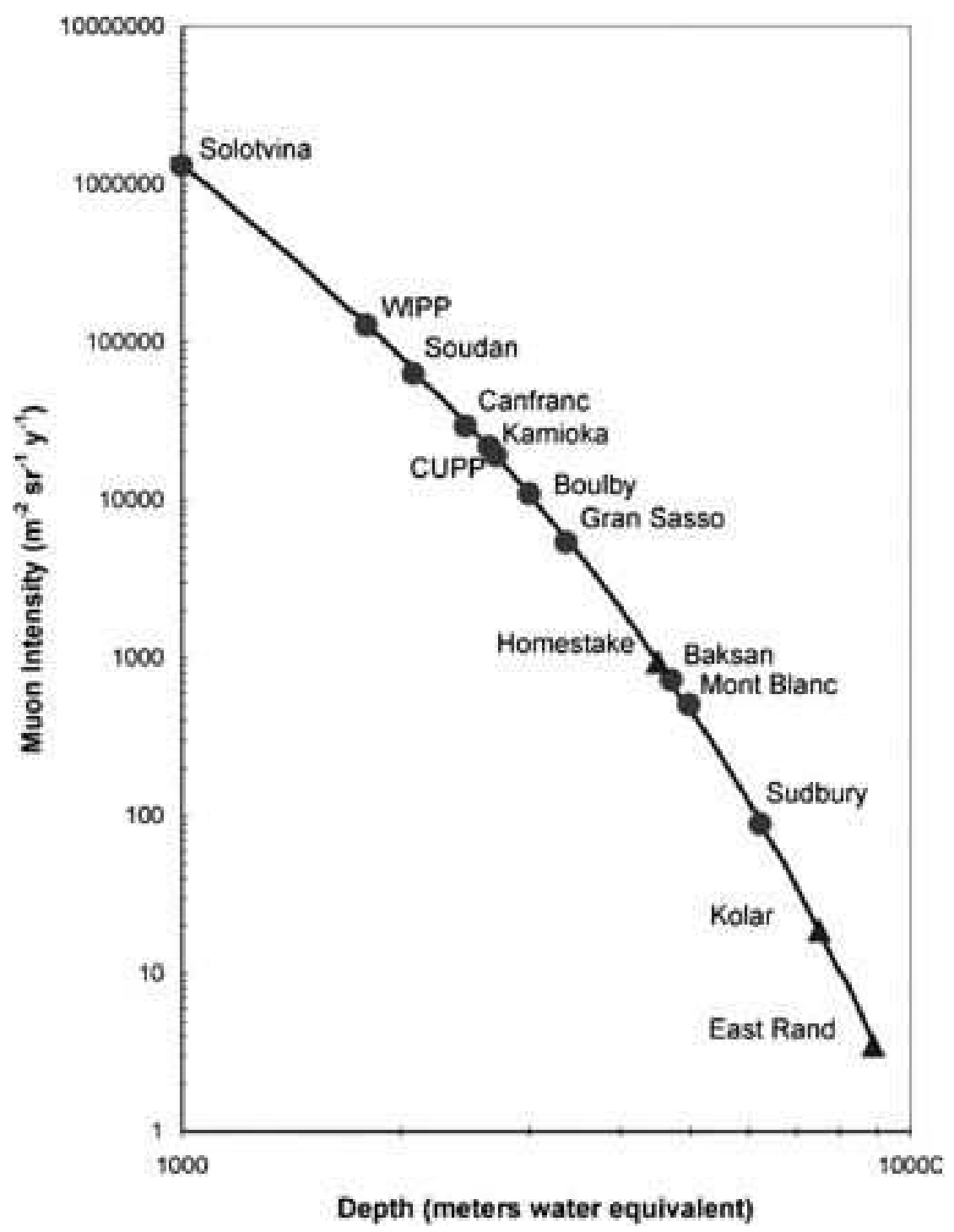

Figura 4.1: Fluxo de múons em função da profundidade em metros de águaequivalente para vários laboratórios subterrâneos e minas ao redor do mundo.

O laboratório oferece uma blindagem de $\sim 3500 \mathrm{~m}$ água-equivalente e foi construído entre as décadas de 70 e 80 quando da construção de um túnel através das montanhas por parte do governo italiano. Essa proteção com rochas pobres em Urânio e Tório reduz o fluxo de raios cósmicos em um milhão e o fluxo de nêutrons em milhares de vezes comparando com a superfície.

Algumas das áreas de pesquisas hospedadas pelo LNGS são: neutrinos solares, neutrinos de SN, oscilação de neutrinos através de feixes advindos do CERN, duplo decaimento beta sem neutrino, procura por matéria escura, reações nucleares e de interesse astrofísico. 


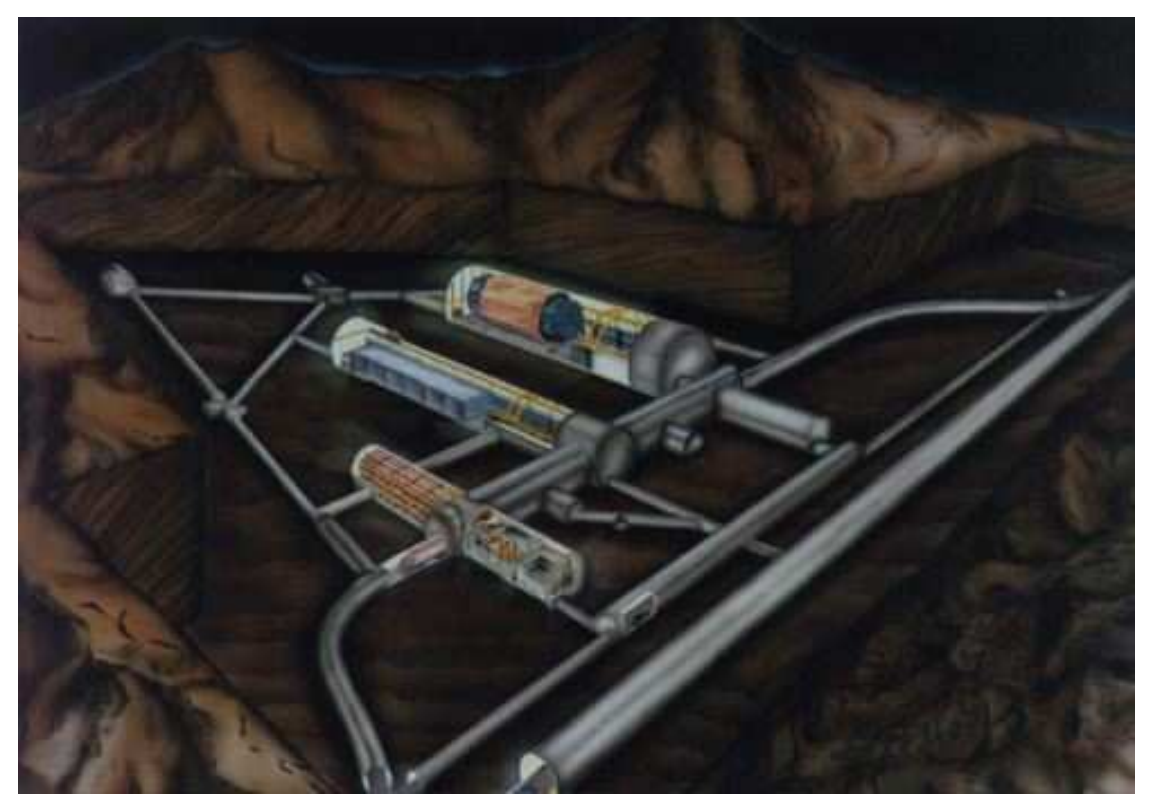

Figura 4.2: Desenho esquemático do Laboratório Nacional de Gran Sasso, na Itália.

\subsection{2}

\section{Kamioka}

O Observatório Subterrâneo de Kamioka começou a ser construído em 1982, sendo finalizado no início de 1983, na mina de Kamioka, no Japão. O objetivo era identificar o decaimento do próton, mas tornou-se famoso principalmente pelo registro dos eventos de neutrinos da SN1987A.

Em 1995, foi extinto o Observatório Subterrâneo de Kamioka e estabelecido o Observatório de Kamioka, com o intuito de avançar com a construção do experimento Super-Kamiokande, que posteriormente ajudou na comprovação da oscilação de neutrinos ao detectar neutrinos atmosféricos [21], que implica que neutrinos tem massa. Apesar de pertencer à Universidade de Tóquio, os experimentos tem a participação de institutos de diversas partes do mundo (EUA, Canadá, Europa, China e Coréia, entre outros). O Observatório de Kamioka é um laboratório essencialmente de física de neutrinos, com diversos experimentos atuando simultaneamente. A Fig. 4.3 mostra um panorama geral sobre os experimentos realizado no Observatório de Kamioka. 


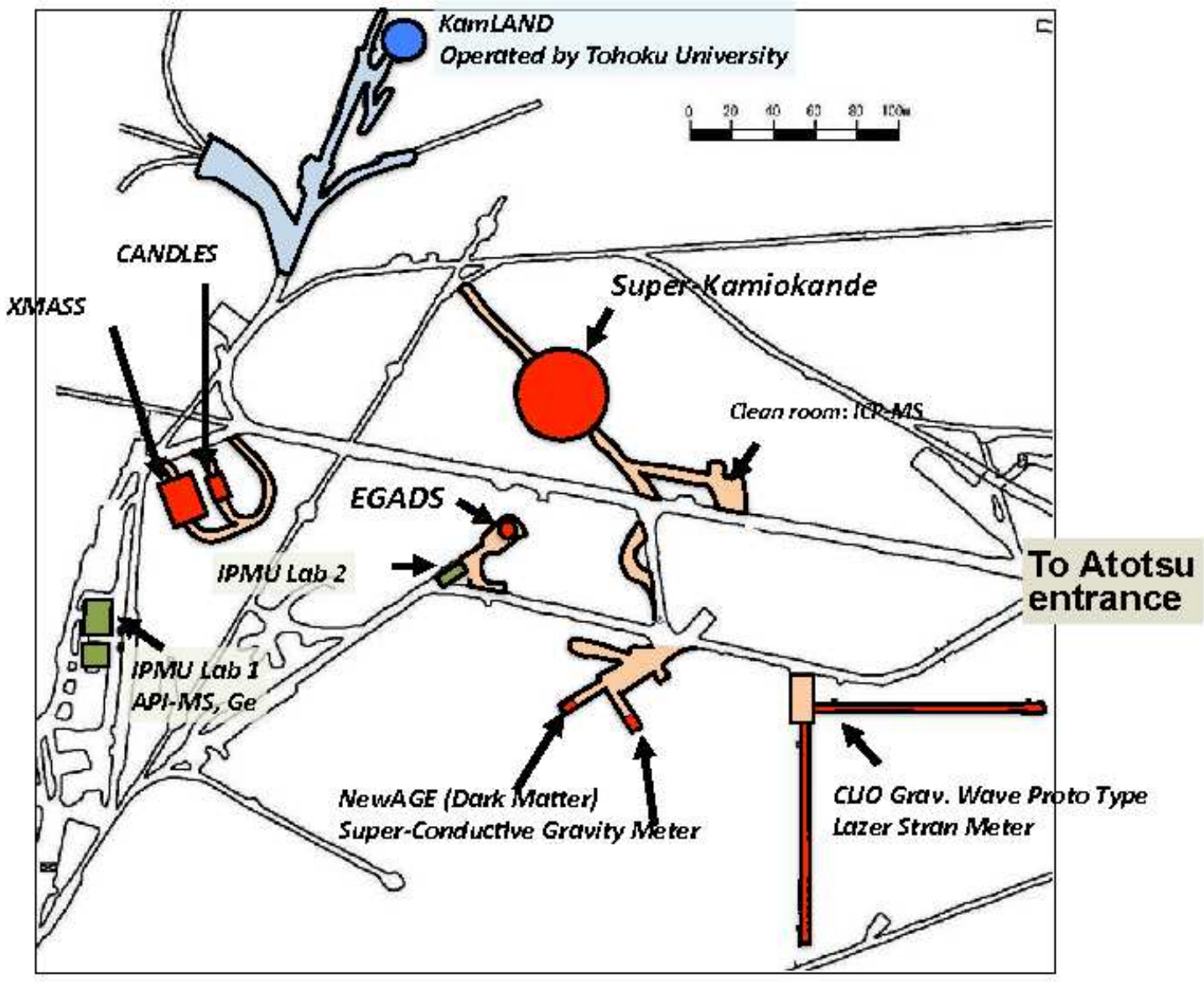

Figura 4.3: Desenho esquemático do Observatório de Kamioka, no Japão, que é administrado pela Universidade de Tóquio, com exceção do experimento KamLAND, que pertence à Universidade de Tohoku.

\subsection{3}

\section{Sudbury Neutrino Observatory}

O Sudbury Neutrino Observatory (SNO) é um observatório de neutrino localizado $\mathrm{a} \sim 2 \mathrm{~km}$ de profundidade ( $\sim 6 \mathrm{~km}$ água-equivalente) na mina Creighton, em Sudbury, no Canadá.

O laboratório consistia de um experimento, de mesmo nome, que operou entre 1999 e 2006 e obteve resultados importantes para solucionar o problema do fluxo de neutrinos solares [121].

Com o fim do projeto, o laboratório foi expandido e renomeado para SNOLAB para que possa receber mais experimentos, conforme mostra a Fig. 4.4, retirada do sítio online do experimento. O SNOLAB é o laboratório subterrâneo mais profundo em operação, tendo $\sim 5 \mathrm{mil} \mathrm{m}^{2}$ de área limpa 


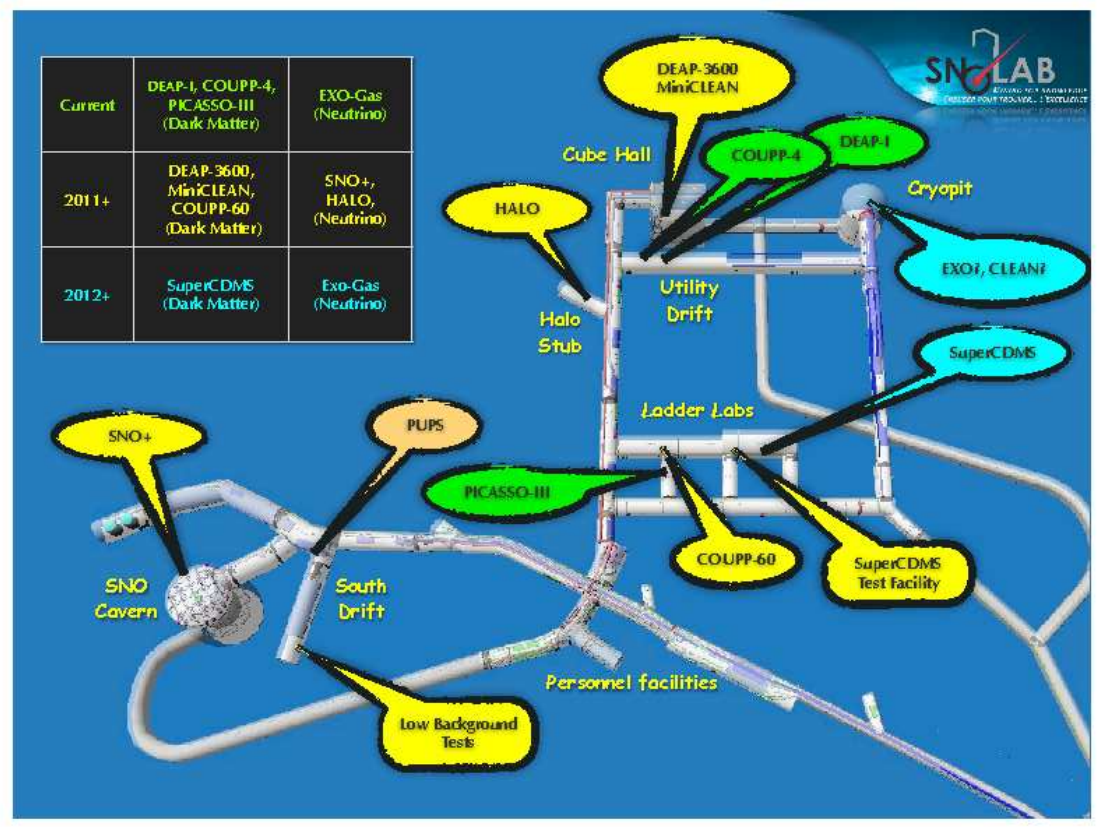

Figura 4.4: Desenho esquemático do laboratório SNOLAB, situado em Sudbury, no Canadá. É o laboratório subterrâneo mais profundo atualmente.

no subsolo. Há em operação, hoje, experimentos de procura por evidência por matéria escura e um experimento para neutrinos de SN que utiliza chumbo como meio de detecção. Há ainda experimentos que entrarão em operação em breve, como é o caso do $\mathrm{SNO}+$, do Enriched Xenon Observatory e as novas fases de experimentos atuais.

\subsection{4}

\section{ANDES}

A Cordilheira dos Andes é a maior cordilheira continental do mundo, alcançando mais de $7000 \mathrm{~km}$ de extensão e acima de $200 \mathrm{~km}$ de largura, ocupando a costa oeste da América do Sul e cruzando sete países. Tais dimensões trazem inúmeros problemas logísticos para o continente, tanto no comércio intra como intercontinental. Com a intenção de suplantar tal barreira, inúmeras vezes cogitou-se a construção de um túnel que interligasse o Oceano Pacífico ao Atlântico. A proposta de criação de um túnel cortando a cordilheira é praticamente tão antiga quanto o próprio Mercado Comum do Sul (MERCOSUL). Entretanto, por motivos diversos, somente em 2005 que começaram os primeiros estudos para averiguar a viabilidade de tal projeto e a partir de 2009 ele é assunto recorrente em encontros do MERCOSUL. Economicamente, a construção do túnel binacional é de suma importância 


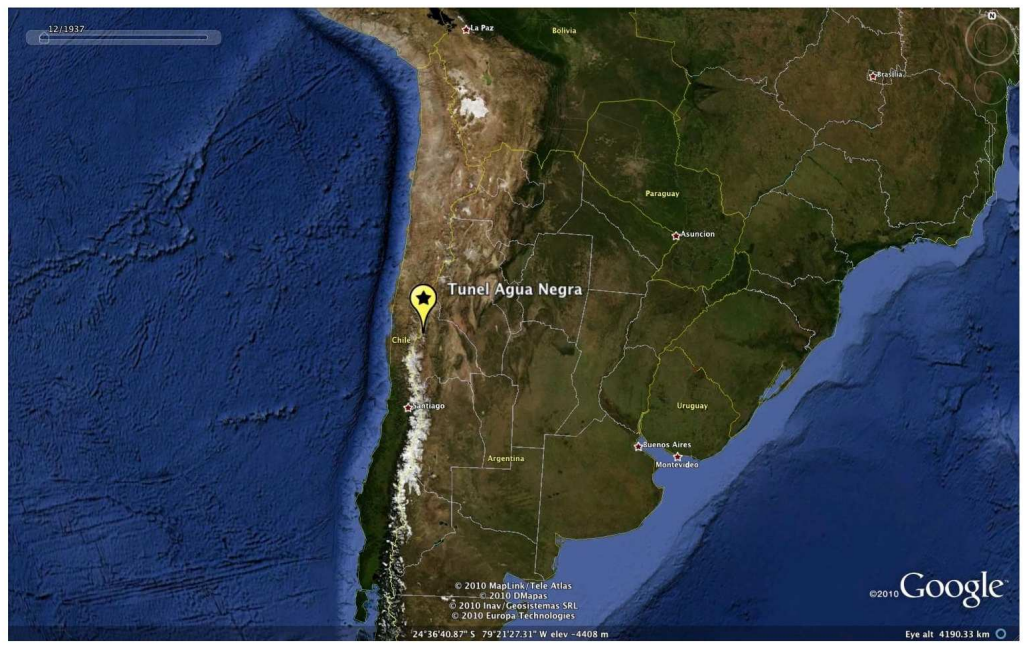

Figura 4.5: Local onde será construído o laboratório de ANDES.

para o escoamento de parte da produção do Brasil e da Argentina através do Chile, facilitando o comércio com a Ásia [24].

Pesquisas indicaram como melhor lugar para a construção a região de Agua Negra, que liga as províncias de San Juan, na Argentina e de Coquimbo, no Chile (Fig. 4.5). Daí o nome do experimento - Agua Negra Deep Experimental Site (ANDES). A previsão de início das obras é para o ano de 2013, tendo duração de sete anos até a conclusão total. Hodiernamente, o projeto prevê dois túneis de $14 \mathrm{~km}$ de extensão, com $12 \mathrm{~m}$ de diâmetro cada, separados por $60 \mathrm{~m}$ entre si, mas conectados a cada $500 \mathrm{~m}$ para acessos de emergências. Outra característica peculiar da obra é que os túneis não serão planos, mas sim em aclive (vindo do Chile), com a entrada no lado chileno situada a 3600 m acima do nível do mar e no lado argentino a saída estará a 4100 m de altitude. A parte mais profunda localizar-se-á próximo da fronteira entre o Chile e a Argentina ( $1750 \mathrm{~m})$, como pode ser visto na Fig. 4.6. Nesta região, a proteção das rochas seria $\geq 4 \mathrm{~km}$ de água-equivalente, uma das maiores da atualidade.

No anseio de aproveitar esta oportunidade ímpar, a comunidade científica da América do Sul, em especial do Brasil, Argentina e Chile, planeja incluir neste projeto a construção do primeiro laboratório subterrâneo no Hemisfério Sul. Para tal, o laboratório subterrâneo deve atender a uma ampla gama de programas científicos, tais como, pesquisa em neutrinos, geoneutrinos, matéria escura, etc.

Para isso, o ANDES terá de ser um complexo de variados laboratórios. O 


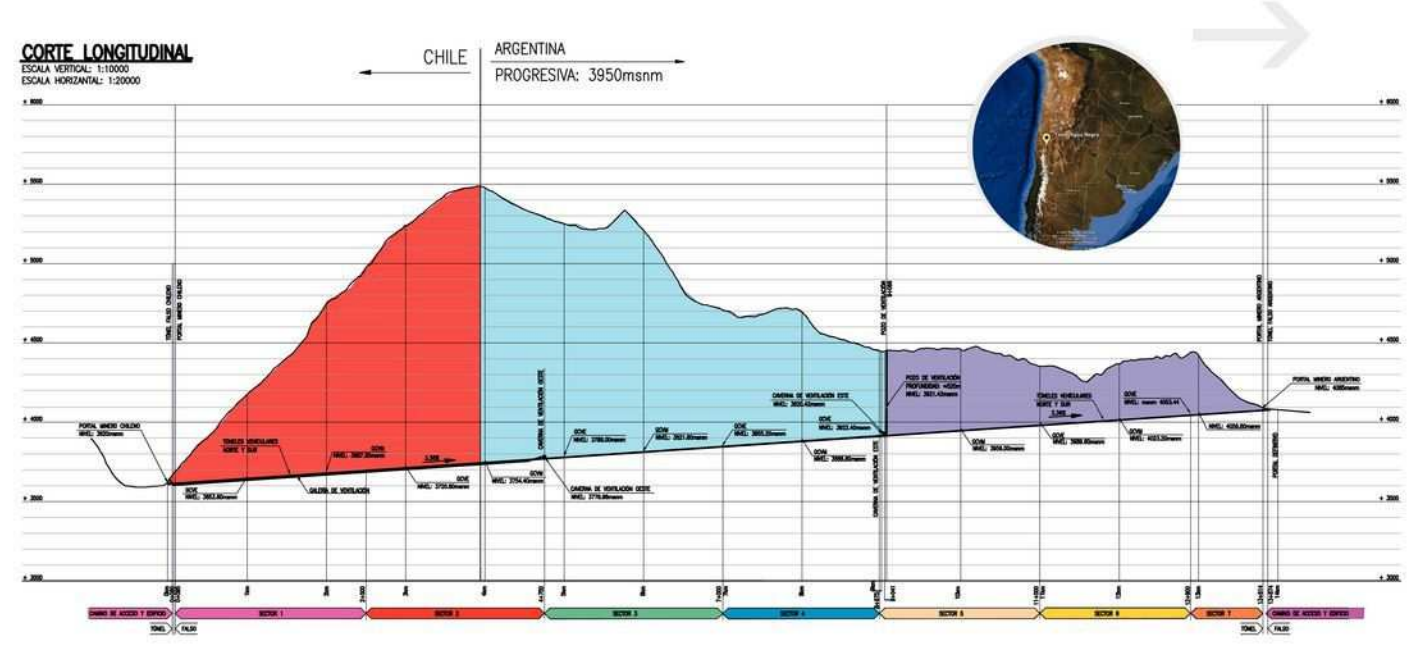

Figura 4.6: Figura esquemática mostrando a diferença de altitude ao longo dos $14 \mathrm{~km}$ de túnel. A fronteira entre o Chile e a Argentina é o melhor local para a construção do laboratório do ponto de vista de blindagem.

design atual do laboratório é mostrado na Fig. 4.7 [23], semelhante a estrutura do já existente LNGS, mas obviamente, com menor tamanho. Serão dois grandes átrios de dimensões 21 x 23 x $50 \mathrm{~m}^{3}$ e 16 x 14 x $40 \mathrm{~m}^{3}$, um átrio menor que atenderá a parte administrativa e experimentos multidisciplinares, cujo tamanho será $17 \times 15 \times 25 \mathrm{~m}^{3}$ e duas cavidades, uma pequena e outra grande. A primeira sediará estudos em radiação ultra-baixa e terá 8 metros de diâmetro e 9 metros de altura, enquanto que a segunda terá 30 metros de diâmetro e 30 metros de altura. Esta cavidade é a de maior interesse do ponto de vista da física de neutrinos porque possui um tamanho apropriado para a instalação de um detector a base de líquido cintilador.

\section{2}

\section{Detectores com Cintilador}

\subsection{1}

\section{Borexino}

O experimento Borexino está localizado no átrio $\mathrm{C}$ do Laboratório Nacional de Gran Sasso (LNGS), na Itália, de acordo com o design mostrado na Fig 4.8 [122].

O Borexino tem como objetivo principal o estudo de propriedades dos neutrinos e embora seu foco principal esteja na detecção de neutrinos solares, 


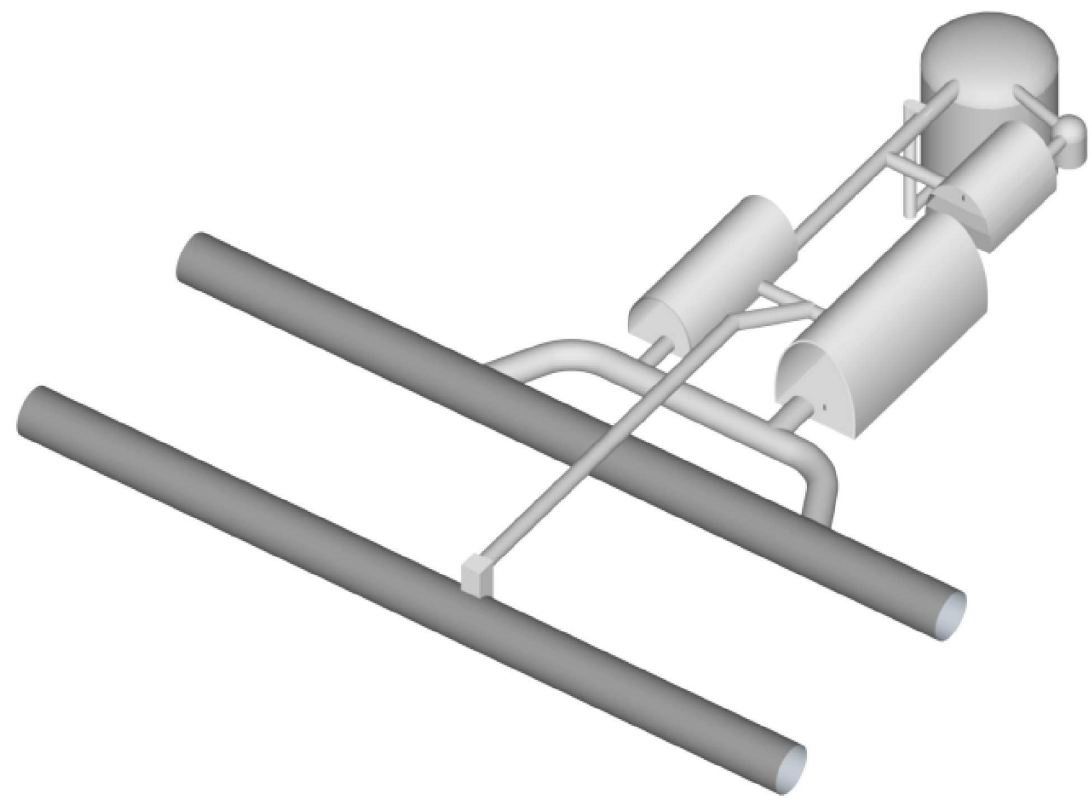

Figura 4.7: Design previsto para o laboratório ANDES. Os dois tubos paralelos são os túneis de tráfego. Após, os três átrios e os dois poços, grande e pequeno. $\mathrm{O}$ poço maior será usado para instalar um detector a base de líquido cintilador.

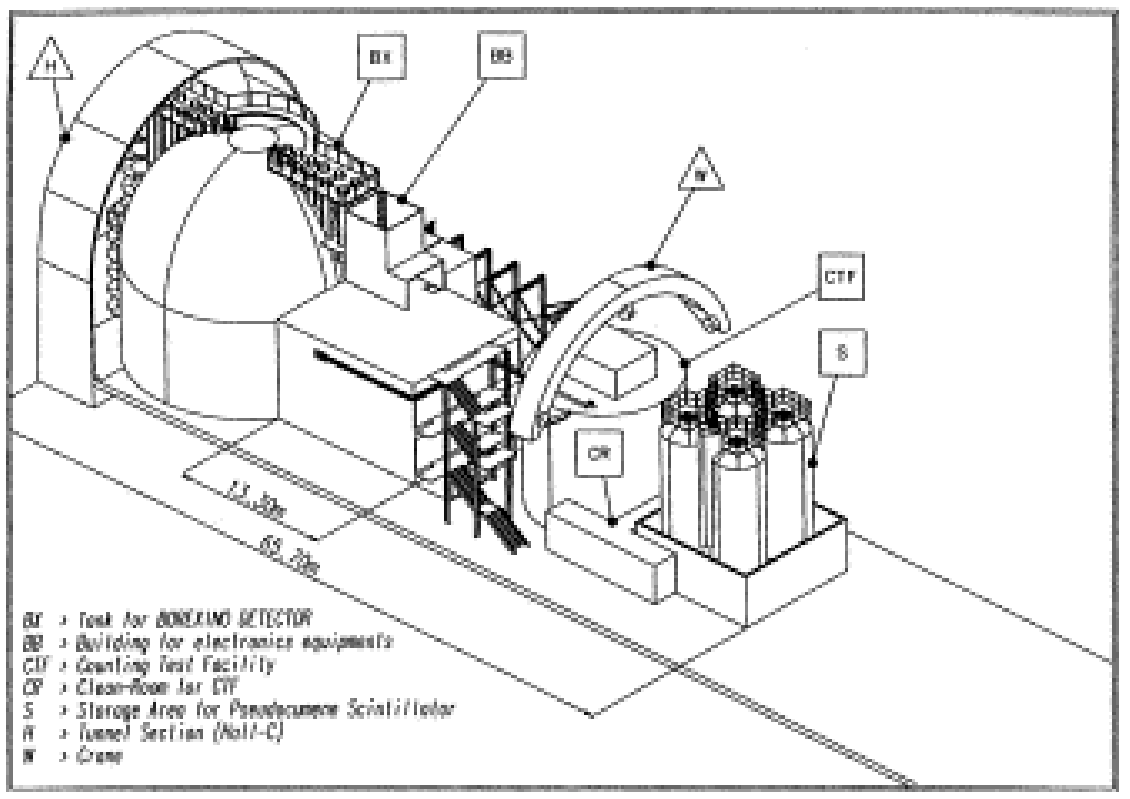

Figura 4.8: Design do experimento Borexino situado na cavidade C do LNGS.

o Borexino também tem capacidade de detecção de neutrinos de supernova.

Ele está em operação desde de 2007 e funciona como detector de tempo real usando o hidrocarboneto aromático 1,2,4-Trimetilbenzeno $\left(\mathrm{C}_{9} \mathrm{H}_{12}\right)$ diluído em óleo mineral como líquido cintilador. O detector tem formato esférico e o seu núcleo é uma esfera de aço inoxidável contendo 300 toneladas de líquido cintilador em um diâmetro de 13,5 m . Deste total, 100 toneladas são de massa 


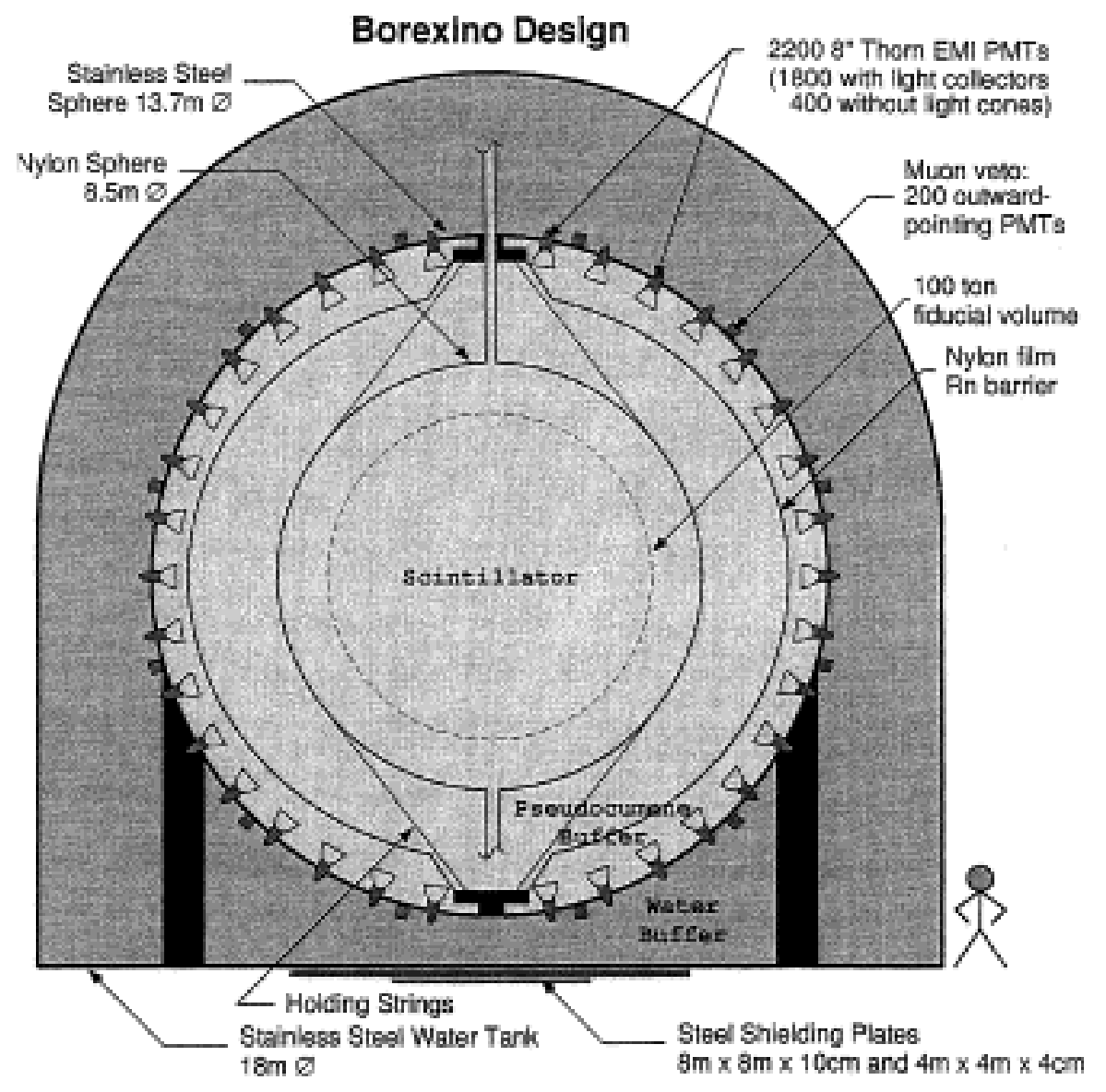

Figura 4.9: Esquete do detector Borexino contendo uma esfera de aço inoxidável, protegida por 1040 toneladas de água pura, para barrar múons atmosféricos.

fiducial $^{1}$ em um diâmetro de 8,5 m. Na esfera de aço estão instaladas 2200 fotomultiplicadoras (PMTs) para realizar a detecção. Para proteger esta esfera de múons atmosféricos, reduzindo ainda mais o ruído do experimento, há um tanque de 18 m de diâmetro preenchido com 1040 toneladas de água pura. Esta descrição pode ser vista na Fig. 4.9 [122].

Para a detecção de uma SN galáctica, o detector Borexino deve registrar $\sim 100$ eventos, a maioria pelo canal da reação de decaimento beta inverso e $\sim 30 \%$ dos eventos devido a interação dos neutrinos com o carbono do líquido cintilador, tanto por CC, como por CN [123].

${ }^{1}$ Massa fiducial é a quantidade de água ou de líquido cintilador que fica na região mais interna do detector onde a maior parte dos eventos de ruídos são excluídos, já que ocorrerão na parte externa do fluido. 


\subsection{2}

\section{KamLAND}

O Kamioka Liquid Scintillator Anti-Neutrino Detector (KamLAND) ocupa atualmente o local onde ficavam as instalações do detector Kamiokande [124], isto é, na mina de Kamioka, no Japão. Ele foi construído com o propósito de observar a oscilação de antineutrinos eletrônicos originados em reatores nucleares, distantes $\sim 180 \mathrm{~km}$, no intuito de solucionar o "problema dos neutrinos solares" ${ }^{2}$ [30].

O detector utiliza um composto de hidrocarbonetos na proporção de $80 \%$ de Dodecano $\left(\mathrm{C}_{12} \mathrm{H}_{26}\right)$ e $20 \%$ de 1,2,4-Trimetilbenzeno $\left(\mathrm{C}_{9} \mathrm{H}_{12}\right)$, totalizando um 1 kt de líquido cintilador.

A estrutura do detector de KamLAND é mostrada na Fig. 4.10 [20]. O líquido cintilador fica contido em uma espécie de balão, de diâmetro de 13 m. Este balão está imerso em um óleo de parafina e de 1,2,4-Trimetilbenzeno. Tudo isto é protegido por uma esfera de aço inoxidável cujo diâmetro é de $18 \mathrm{~m}$ e é onde estão instaladas 1879 PMTs. Este é o detector interno. A esfera de aço inoxidável, por sua vez, é envolta por 3,2 kt de água pura, que faz a blindagem devido a radiação de rochas, múons de raios cósmicos. Nas bordas deste tanque com água estão instaladas 225 PMTs, formando o detector externo. A detecção se dá através de radiação Cherenkov.

\section{2 .3}

$\mathrm{SNO}+$

O detector Sudbury Neutrino Observatory (SNO) foi construído para estudar propriedades de massa e mistura de neutrinos, em especial, o fenômeno de oscilação de neutrinos ao observar neutrinos solares - na tentativa de solucionar o problema dos neutrinos solares [125].

Localizado na mina Creighton, em Sudbury, no Canadá, o SNO era um detector baseado em radiação Cherenkov que usava $1 \mathrm{kt}$ de água pesada $\left(\mathrm{D}_{2} \mathrm{O}\right)$ ao invés de líquido cintilador como material de detecção. A preferência por água pesada em detrimento da água pura, utilizada em outros detectores é porque com aquela é possível detectar interações tanto de corrente neutra como de corrente carregada. A proteção contra múons de raios cósmicos oferecida

\footnotetext{
${ }^{2}$ Resumidamente, podemos dizer que era a diferença entre o fluxo de neutrinos previstos teoricamente e o observado experimentalmente.
} 


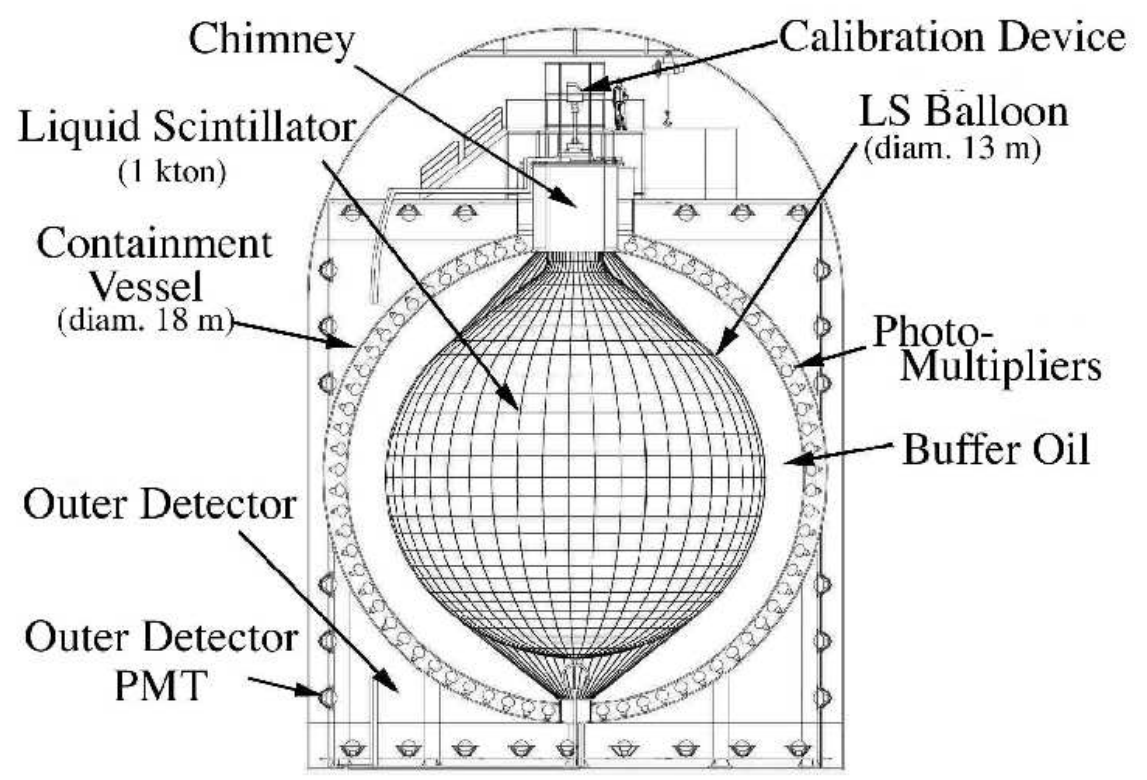

Figura 4.10: Diagrama esquemático do detector de KamLAND.

pela mina é uma das melhores no mundo, aproximadamente 6000 m de águaequivalente.

Na Fig. 4.11 está uma visão geral do laboratório de SNO, com o detector, sala de controle, locais de armazenamento e processamento de água pura e pesada, entre outros. O detector, em si, é mostrado na Fig. 4.12 [125] no local escavado especialmente para a montagem do dispositivo, com $22 \mathrm{~m}$ de diâmetro e $34 \mathrm{~m}$ de altura. Uma esfera de acrílico de $12 \mathrm{~m}$ de diâmetro continha $1 \mathrm{kt}$ de água pesada ultrapura. Concêntrica à esfera de acrílico, fez-se uma estrutura esférica de aço inoxidável, cujo diâmetro de 17,8 m armazenava água pura para proteger o detector interno. Nesta estrutura foram instaladas 9438 PMTs e o espaço externo também foi preenchido com água pura.

Como é de conhecimento popular, o experimento SNO foi de grande sucesso no seu objetivo principal, confirmando, de maneira independente, a oscilação de neutrinos e de que não há déficit no fluxo total de neutrinos solares [121, 126]. O experimento operou por um período de 7 anos e meio 1999 até 2006 - quando foi desativado.

Em 2010, o governo canadense aprovou o sucessor do experimento SNO, o SNO+. Ele usará as mesmas instalações de seu antecessor, mas haverá grandes mudanças para receber o novo experimento. Obviamente, o objetivo principal do novo experimento é outro. Agora, o desafio do SNO+ é a observação do duplo decaimento beta sem neutrinos $(0 \nu \beta \beta)$, que se 


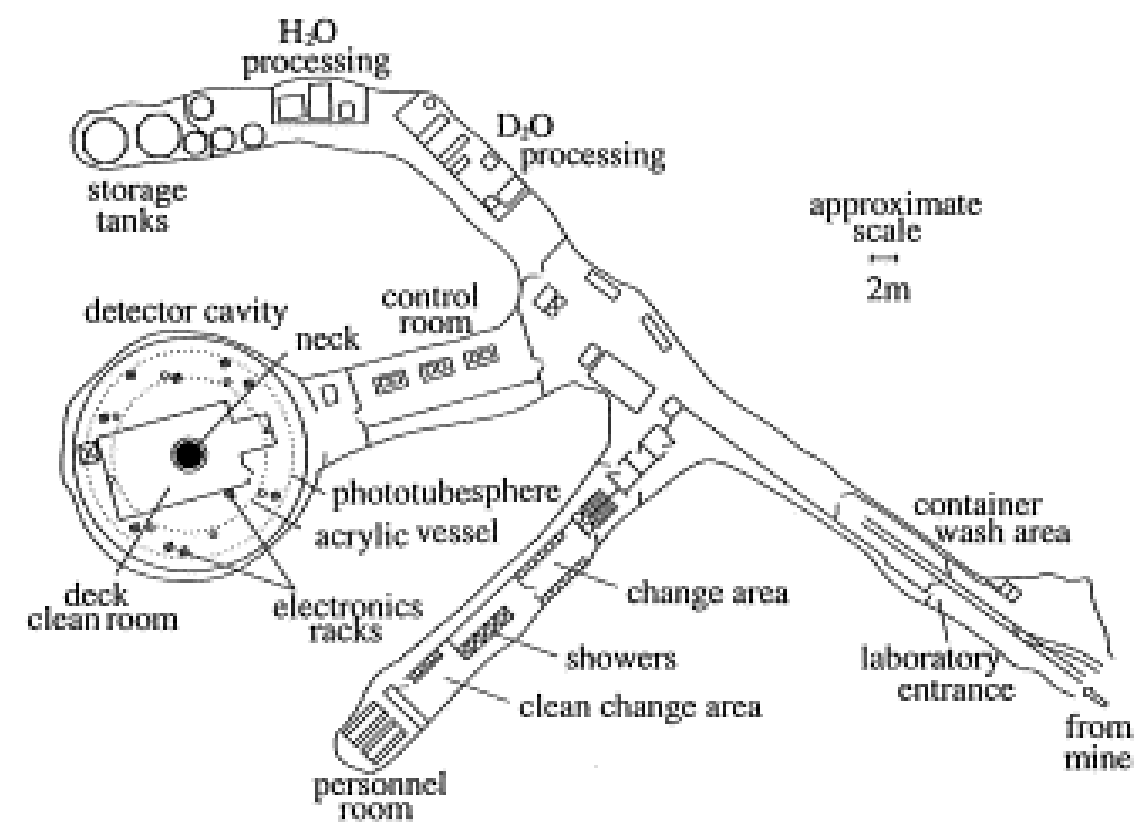

Figura 4.11: Panorama do laboratório SNO, situado há 2000m de profundidade na mina Creighton, em Sudbury, Canadá. Agora será a casa do experimento SNO+.

comprovada a existência, nos indica a natureza dos neutrinos como partículas de Majorana [127, 128]. Entretanto, o SNO+ também terá "olhos" para outras medidas envolvendo neutrinos, tais como: neutrinos solares de baixa energia (sub-MeV), geoneutrinos ${ }^{3}$, neutrinos de reatores e neutrinos de supernovas galácticas [128]. Nesta última, espera-se um total de $\sim 600$ eventos (CC e CN) para uma SN distante $10 \mathrm{kpc}$ da Terra.

Apesar de ser usada a mesma estrutura mostrada na Fig. 4.12, o SNO+ não usará água pesada como meio de detecção. Ao invés disto, usará 1 kt de líquido cintilador. E o escolhido foi o Alquilbenzeno Linear $\left(\mathrm{C}_{6} \mathrm{H}_{5} \mathrm{C}_{12} \mathrm{H}_{25}\right)$ devido ao seu baixo custo, taxa relativamente alta de produção de luz, compatibilidade com a esfera de acrílico e alta estabilidade ( $>2$ anos) [128].

\subsection{4}

\section{ANDES}

O detector de neutrinos do laboratório de ANDES pode ser instalado na maior cavidade do laboratório, conforme Fig. 4.7. Com tais dimensões, tornase inviável construir um detector a base de água. Embora seja mais barato que líquido cintilador, o ANDES teria um tamanho muito diminuto comparado aos

\footnotetext{
${ }^{3}$ Geoneutrinos são os neutrinos produzidos no interior da Terra devido ao decaimento beta que ocorre em elementos radioativos como Urânio, Tório e, em maior escala - mas menor energia - o Potássio. Ele responde por grande parte do calor emitido pela Terra.
} 


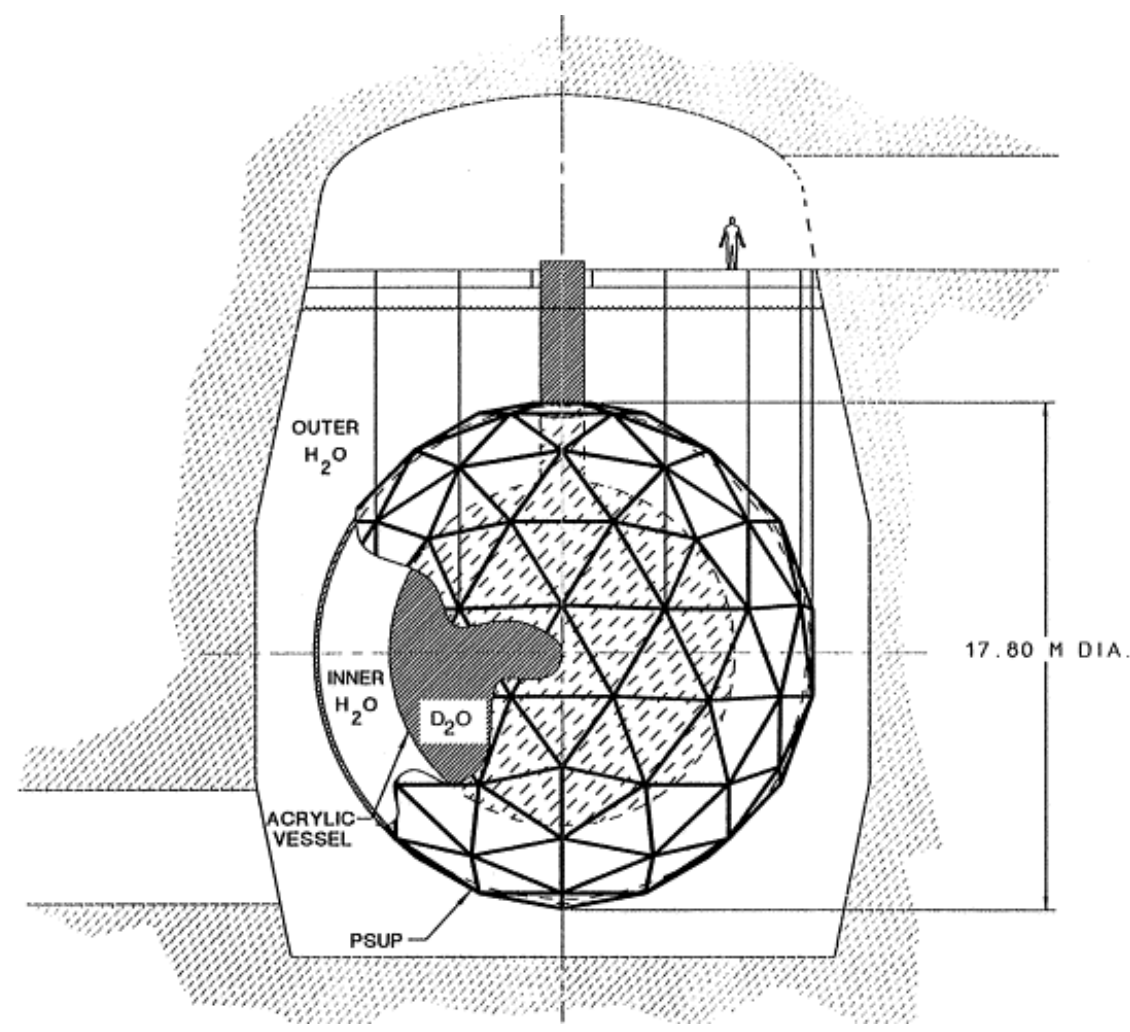

Figura 4.12: O detector do laboratório SNO localizado na cavidade feita exclusivamente para ele. Toda estrutura será reaproveitada pelo experimento $\mathrm{SNO}+$, que substituirá a água pesada por líquido cintilador como meio para detecção.

grandes detectores existentes a base de água, que utilizam radiação Cherenkov como detecção. Assim, a melhor hipótese é utilizar líquido cintilador, também fato de que este possui uma grande capacidade de observar antineutrinos eletrônicos através da reação de decaimento beta inverso $\left(\bar{\nu}_{e}+p \rightarrow n+e^{+}\right)$, excelente resolução energética e baixo limite inferior de energia.

$\mathrm{Na}$ escolha dos possíveis candidatos a líquido cintilador do detector de ANDES, escolhemos compostos atualmente usados ou planejados para detectores de tamanhos similares ao ANDES - KamLAND, Borexino e SNO+.

As principais reações, i.e., que mais produzem eventos, para um detector a base de líquido cintilador são: i) decaimento beta inverso e; ii) espalhamento elástico neutrino-próton. Vamos falar sucintamente como calcular o número de eventos em cada um destes dois canais de reação do neutrino.

\section{Decaimento beta inverso}

Podemos calcular o número de eventos do decaimento beta inverso, $\bar{\nu}_{e}+p \rightarrow n+e^{+}$, é dado por 


$$
N=N_{p} \int_{E_{\min }}^{\infty} d E F_{\bar{\nu}_{e}}(E) \sigma_{\bar{\nu}_{e} p}(E)
$$

onde $E_{\min }=1.806 \mathrm{MeV}$ é o limite inferior desta reação, $E$ é a energia observada e $N_{p}$ é o número de prótons livres do detector - no caso de ANDES com $3 \mathrm{kt}$ de cintilador Alquilbenzeno, é $2.2 \times 10^{32}$. A seção de choque do $\bar{\nu}_{e}$ com o próton depende da energia do neutrino e pode ser escrita como [129]

$$
\sigma_{\bar{\nu}_{e} p}(E) \approx p_{e} E_{e} E^{-0.07056+0.02018 \ln E-0.001953 \ln ^{3} E} \times 10^{-43}\left[\mathrm{~cm}^{2}\right]
$$

onde $E_{e}=E-\left(m_{n}-m_{p}\right) \simeq E-1.293 \mathrm{MeV}\left(m_{p}, m_{n}\right.$ são as massas do próton e do nêutron, respectivamente), e $p_{e}$ é o momento do pósitron. Todas as energias são dadas em MeV.

\section{Espalhamento elástico neutrino-próton}

Esta reação é válida para todos os sabores de neutrinos, o que significa que não há significância o efeito de oscilação dos neutrinos. A seção de choque diferencial do $\nu+p \rightarrow \nu+p$ é dada por

$$
\frac{d \sigma}{d T}=\frac{G_{F}^{2} m_{p}}{\pi}\left[\left(1-\frac{m_{p} T}{2 E^{2}}\right) c_{v}^{2}+\left(1+\frac{m_{p} T}{2 E^{2}}\right) c_{a}^{2}\right]
$$

onde $G_{F}$ é a constante de Fermi, $m_{p}$ é a massa do próton, $T$ é a energia cinética de recúo do próton, $E$ é a energia do neutrino, $c_{v}=0.04$ e $c_{a}=1.27 / 2$ são as constantes de acoplamento para corrente neutra. Levando em conta a perda de energia do próton, calculamos a energia de extinção do próton, $T^{\prime}$, através

$$
T^{\prime}(T)=\int_{0}^{T} \frac{d T}{1+k_{B}\langle d T / d x\rangle},
$$

onde $k_{B}$ é conhecido como constante de Birk. O valor numérico de $\langle d T / d x\rangle$ que descreve a perda de energia do próton no cintilador foi retirado da Ref. [130].

A distribuição do número de eventos em função da energia de extinção, $d N / d T^{\prime}$, é calculada como

$$
\frac{d N}{d T^{\prime}}=N_{p}\left(\frac{d T^{\prime}}{d T}\right)^{-1} \int_{E_{\min }}^{\infty} d E \frac{d F}{d E} \frac{d \sigma}{d T},
$$

onde $E_{\text {min }}$ é a energia mínima do neutrino que pode causar o recuo do próton com a energia cinética $T$. $E_{\text {min }}$ é dado por, 


$$
E_{\min }=\frac{1}{2}\left[T+\sqrt{T\left(T+2 m_{p}\right)}\right] \simeq \sqrt{\frac{m_{p} T}{2}} .
$$

Levando em conta a resolução energética para converter $d N / d T^{\prime}$ no valor que é realmente observado, $d N / d T_{\text {que }}$ fazemos

$$
\frac{d N}{d T_{\text {que }}}=\int_{0}^{\infty} d T^{\prime} \frac{d N}{d T^{\prime}} R\left(T_{\text {que }}, T^{\prime}\right),
$$

onde $R\left(T_{\text {que }}, T^{\prime}\right)$ é a função resolução, dada por

$$
R\left(T_{\text {que }}, T^{\prime}\right)=\frac{1}{\sqrt{2 \pi} \sigma} \exp \left[-\frac{\left(T_{\text {que }}-T^{\prime}\right)^{2}}{2 \sigma^{2}}\right]
$$

onde iremos assumir a resolução como $5 \% / \sqrt{T^{\prime} / \mathrm{MeV}}$ [131].

\section{3}

\section{Por que ANDES?}

A ideia do laboratório de ANDES, pela comunidade científica sulamericana, surgiu após a aprovação da construção do túnel de Água Negra, que ligará o Chile à Argentina sob a Cordilheira dos Andes. É a oportunidade ideal para a construção do primeiro laboratório subterrâneo do Hemisfério Sul.

Além de ser um marco, há a importância científica por trás deste anseio. A maioria dos laboratórios subterrâneos do Hemisfério Norte encontram-se em regiões onde também há muitas usinas nucleares, que produzem antineutrinos e são ruídos quando queremos observar neutrinos de outras fontes, como os geoneutrinos.

A América do Sul, de um modo geral, praticamente não possui reatores nucleares, o que torna a região livre de uma grande parte dos ruídos, propiciando excelente condição para detecção de geoneutrinos. Urânio e Tório são os principais produtores de geoneutrinos na faixa de energia possível de detectar $(\sim 1.8-3.5 \mathrm{MeV})$. Eles os produzem via decaimento $\beta$. E, como a maior concentração destes elementos está na crosta terrestre, quanto mais espessa é esta camada, maior é o fluxo. Na Fig. 4.13 [132] podemos ver o fluxo de geoneutrinos na Terra. Observe que as maiores taxas estão nas regiões montanhosas do planeta, incluindo a Cordilheira dos Andes. Assim, o laboratório de ANDES está em um local privilegiado para realizar este trabalho. Na Fig. 4.14 [22] podemos ver o fluxo esperado para o laboratório de ANDES separando a contribuição do manto e da crosta. Além disso, também há o fluxo esperado para outros lugares. Os pontos em preto indicam resultado de medidas de KamLAND e 


\begin{tabular}{|l|c|c|c||c|}
\hline Localização & Número devido U & Número devido Th & Total & TNU \\
\hline Gran Sasso & 53.8 & 14.7 & 68.5 & 45.5 \\
Kamioka & 45.7 & 12.4 & 58.1 & 32.9 \\
Hawaii & 18.5 & 5.0 & 23.5 & 13.0 \\
Sudbury & 63.2 & 17.2 & 80.4 & 45.5 \\
Pyhäsalmi & 66.1 & 18.0 & 84.1 & 47.6 \\
ANDES & 64.8 & 17.6 & 82.4 & 46.8 \\
\hline
\end{tabular}

Tabela 4.1: Número esperado de eventos de geoneutrinos para o detector de ANDES com 3 kt de líquido cintilador após um ano de operação com $80 \%$ de eficiência considerando diferentes lugares na Terra. TNU significa terrestrial neutrino units.

Borexino. O ponto em azul, refere-se ao fluxo medido esperado para ANDES após 5 anos de tomada de dados.

A tabela 4.1 [22] quantifica o número total de eventos de geoneutrinos em vários laboratórios separando a contribuição do Tório e do Urânio. Note que a Cordilheira dos Andes possui um dos maiores fluxos e, considerando a ausência de ruído devido a neutrinos de reatores, a probabilidade de detecção torna-se maior.

O laboratório de ANDES também pode contribuir na procura por matéria escura. Acredita-se que a interação de partículas de matéria escura possam interagir fracamente com a matéria usual. Um modo de observar esta interação é o espalhamento elástico com o núcleo de um átomo. Calculando a energia depositada pela partícula de matéria escura através do recuo do núcleo do átomo. Outra forma de procurar por evidência de matéria escura é a modulação do sinal considerando o movimento da Terra ao redor do Sol. Independente do método, é necessário levar estes experimentos para ambientes

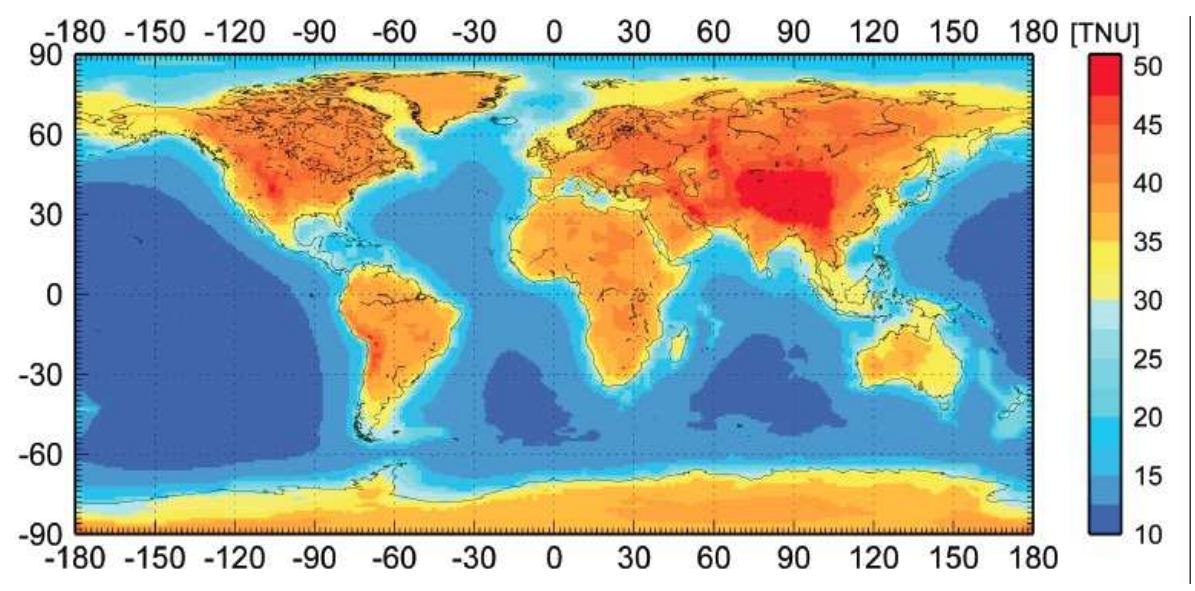

Figura 4.13: Distribuição do fluxo de geoneutrinos na Terra. 


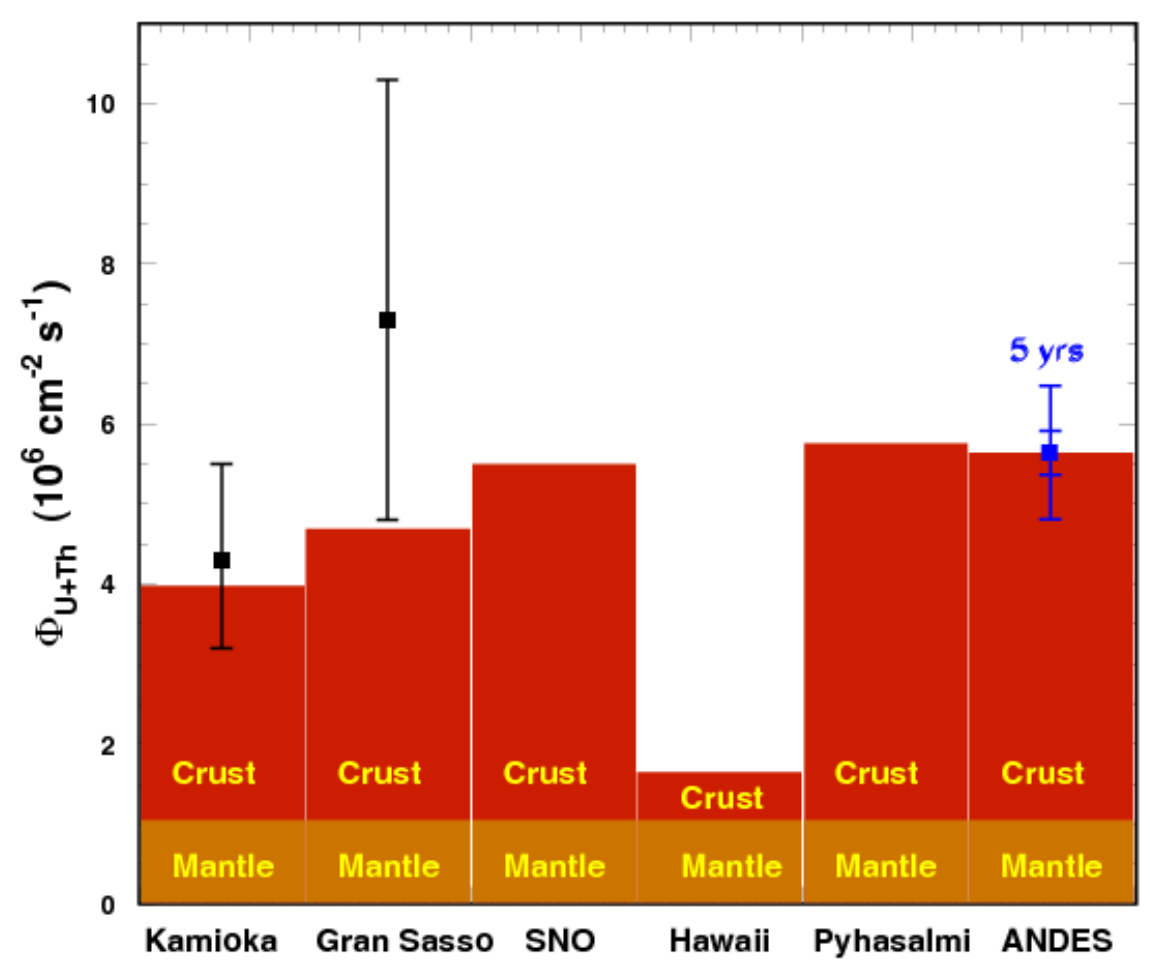

Figura 4.14: Fluxo total de geoneutrinos esperados em vários locais da Terra, separando a contribuição do manto e da crosta. O ponto em azul mostra o fluxo esperado para o detector de ANDES após 5 anos de tomada de dados com precisão de 1 e $3 \sigma$ de nível de confiança.

livres de qualquer ruído, já que são sinais muito fracos.

A próxima geração de interferômetros para a detecção de ondas gravitacionais poderá ir ao subsolo, com a supressão do ruído, tanto de fenômenos atmosféricos como humanos. Neste quesito o laboratório de ANDES também tem vantagem, já que a blindagem será uma das melhores existentes.

O estudo de fenômenos geodinâmicos em baixas frequências também só é possível em condições especiais, com pouco barulho. O estudo de microorganismos que podem sobreviver em condições extremas de pressão e temperatura, por exemplo, também tem lugar no laboratório de ANDES.

Finalmente, a observação de neutrinos vindos da próxima SN galáctica é possível com o ANDES. Como a ocorrência de SN locais são eventos raros ( $1-3$ por século), precisamos estar preparados para quando ocorrer a próxima explosão. O detector de ANDES ainda pode vir a ser membro do SuperNova Early Warning System (SNEWS) [133], que é uma rede 
internacional para avisar aos astrônomos quando da ocorrência de uma SN. Desse modo, os telescópios terrestres podem ser direcionados em tempo de observar o surgimento da curva de luz da explosão estelar. Vários detectores fazem parte do SNEWS: Super-Kamiokande, LVD, KamLAND, IceCube, Borexino entre outros. Como o foco do nosso trabalho é o potencial de detecção de neutrinos de SN e o que pode ser inferido, iremos apresentar os resultados de maneira mais detalhada no próximo capítulo. 


\section{Resultados}

Neste capítulo iremos apresentar as suposições feitas em relação a ocorrência de uma SN galáctica e os resultados do que pode ser observado no detector de ANDES.

\section{1 \\ Metodologia}

Hodiernamente estamos muito melhor preparados para observar a ocorrência de uma SN do que estávamos em 1987. Infelizmente a taxa de SN é baixa na Via Láctea, $\leq 3$ por século [134, 43, 135]. Assim, embora há grandes detectores, como o Super-Kamiokande [136] e o IceCube [137] em operação, é melhor que tenhamos o máximo de detectores possível, para registrarmos a maior quantidade de informações que pudermos sobre neutrinos de SN. Isto nos ajudará para uma compreensão melhor sobre o mecanismo de explosão e dinâmica de supernovas.

A existência de um número maior de detectores de neutrinos permitirá uma resposta melhor do sistema de alerta aos astrônomos sobre a ocorrência de uma SN antes da chegada dos fótons à Terra [133]. Este alarme permitirá que os telescópios ao redor do planeta registrem o início da curva de luz da SN, uma vez que eles já saberão onde houve a explosão.

$\mathrm{Na}$ realização deste trabalho, utilizamos a distribuição do grupo de Garching [138, 78, 139] para representar o espectro de energia dos neutrinos de SN quando da chegada destes à Terra, sem levar em conta a oscilação de neutrinos,

$$
F_{\nu_{\alpha}}^{0}(E)=\frac{1}{4 \pi D^{2}} \frac{\Phi_{\nu_{\alpha}}}{\left\langle E_{\nu_{\alpha}}\right\rangle} \frac{\beta_{\alpha}^{\beta_{\alpha}}}{\Gamma\left(\beta_{\alpha}\right)}\left[\frac{E}{\left\langle E_{\nu_{\alpha}}\right\rangle}\right]^{\beta_{\alpha}-1} \exp \left[-\beta_{\alpha} \frac{E}{\left\langle E_{\nu_{\alpha}}\right\rangle}\right]
$$

onde $\mathrm{D}$ é a distância da Terra à $\mathrm{SN}, \Phi_{\nu_{\alpha}}$ é o número total de neutrinos de 
sabor $\alpha$ emitidos, $\left\langle E_{\nu_{\alpha}}\right\rangle$ é a energia média do neutrino de sabor $\alpha, \Gamma\left(\beta_{\alpha}\right)$ é a função gama e $\beta_{\alpha}$ é um parâmetro para descrever o desvio em relação a um espectro térmico, cujo valor adotado neste trabalho foi $\beta=4$.

A energia emitida na forma de neutrinos em uma explosão de SN é $\sim 3 \times 10^{53}$ erg e consideramos que seja igualmente distribuída entre todos os sabores.

A distância adotada em nossos cálculos foi de $10 \mathrm{kpc}$, que é a distância típica adotada na literatura. Entretanto, a SN pode ocorrer mais próximo da Terra, oferecendo um fluxo maior de neutrinos.

Como sabemos, neutrinos oscilam [21, 121], assim o fluxo é diferente do mostrado na eq. (5.1). O fluxo do neutrino eletrônico que chegará na Terra após a oscilação dentro da SN pode ser expresso como vimos no cap. 3, eq. (3.121),

$$
F_{\bar{\nu}_{e}}(E)=\bar{p}(E) F_{\bar{\nu}_{e}}^{0}(E)+[1-\bar{p}(E)] F_{\bar{\nu}_{x}}^{0}(E)
$$

onde o índice 0 significa original, no local de criação (na neutrinosfera), antes de oscilar e o subíndice $x$ refere-se aos antineutrinos do múon e tau ou uma combinação linear destes, permitindo que façamos análises em 2 sabores. A função $\bar{p}(E)$ é a probabilidade de sobrevivência do antineutrino eletrônico. Ela depende da energia do neutrino e engloba todos os efeitos de oscilação que ocorrem dentro da SN: efeito MSW, efeitos coletivos, turbulências, etc [111, 140, 141, 142, 143].

Da eq. (5.2), podemos perceber que dado um $\bar{p}(E) \neq 1$, quanto maior for a diferença entre os fluxos $F_{\bar{\nu}_{e}}^{0}(E)$ e $F_{\bar{\nu}_{x}}^{0}(E)$, maior será o efeito observável na Terra. Além do caso de $\bar{p}(E)=1$, se $F_{\bar{\nu}_{e}}^{0}(E)=F_{\bar{\nu}_{x}}^{0}(E)$, também não é possível observarmos qualquer efeito.

Apesar de efeitos como oscilações coletivas ${ }^{1}$, ondas de choques e turbulências afetarem bastante a determinação de $\bar{p}(E)$ no caso da hierarquia invertida, alguns estudos [145, 146] indicam que efeitos coletivos são fortemente suprimidos devido a alta densidade da matéria, fazendo com que apenas o efeito MSW seja determinante no cálculo de $\bar{p}(E)$. Então, para a hierarquia normal a probabilidade de sobrevivência do antineutrino eletrônico é dada pela Eq. (3.124) e para a hierarquia invertida, a Eq. (3.137) resulta em $\bar{p} \simeq 0$. Dessa

\footnotetext{
${ }^{1}$ Oscilações coletivas são oscilações de neutrinos que podem ocorrer no interior das estrelas devido a interação neutrino-neutrino, uma vez que a densidade é elevada e os neutrinos estão "presos' . Veja, por exemplo, a Ref. [144] para uma revisão sobre o assunto.
} 


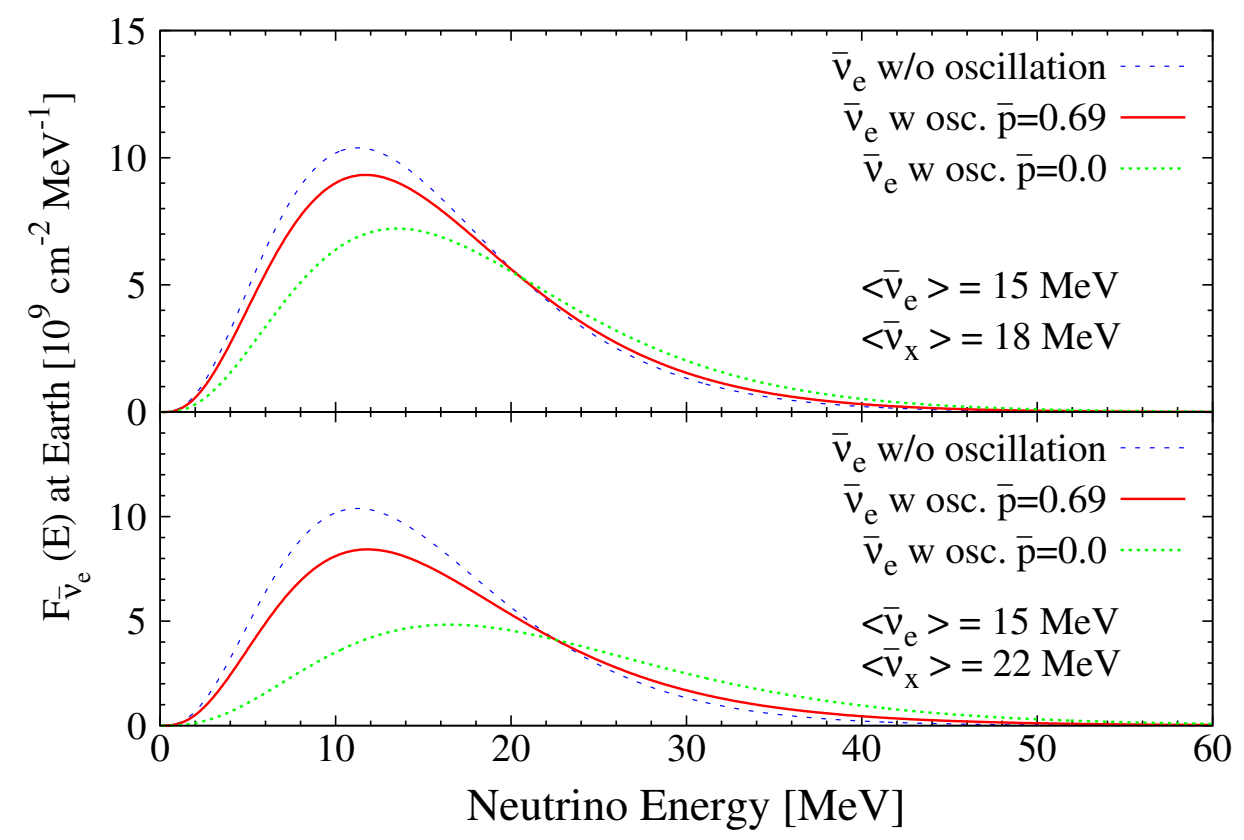

Figura 5.1: Fluxos de antineutrinos eletrônicos esperados na Terra para a ocorrência de um SN a $10 \mathrm{kpc}$ de distância. No painel superior, $\left\langle E_{\bar{\nu}_{x}}\right\rangle=18 \mathrm{MeV}$, enquanto que no inferior $\left\langle E_{\bar{\nu}_{x}}\right\rangle=22 \mathrm{MeV}$. Em ambos, $\left\langle E_{\bar{\nu}_{e}}\right\rangle=15 \mathrm{MeV}$.

forma, independentemente da hierarquia de massa e dos efeitos de correções levados em conta, temos

$$
0 \leq \bar{p}(E) \leq \cos ^{2} \theta_{12} \simeq 0.69
$$

Assim, o espectro do fluxo de neutrinos para uma SN que ocorra a $10 \mathrm{kpc}$ da Terra tem a forma mostrada na Fig. 5.1, onde usamos os valores típicos para as energias médias dos neutrinos, isto é, $\left\langle E_{\bar{\nu}_{e}}\right\rangle=15 \mathrm{MeV}$ e $\left\langle E_{\bar{\nu}_{x}}\right\rangle=18 \mathrm{MeV}$ e $22 \mathrm{MeV}$, nos painéis superior e inferior, respectivamente. Nesta figura, a linha vermelha indica o fluxo do $\bar{\nu}_{e}$ que seria observado na Terra para hierarquia normal, dado pela Eq. (5.2), enquanto que a linha verde refere-se a hierarquia invertida, onde $\bar{p}(E)$ é nulo. Ainda da Fig. 5.1, percebemos que quanto maior for a energia média, menor será o fluxo dos neutrinos, já que a energia total mantém-se igual.

Embora existam vários canais de reação dos neutrinos com a matéria, usaremos os dois principais: decaimento beta inverso $\left(\bar{\nu}_{e}+p \rightarrow n+e^{+}\right)$e o espalhamento elástico entre o próton e o neutrino $(\nu+p \rightarrow \nu+p)$. O primeiro tem a maior seção de choque para reações com neutrinos e o segundo é útil para determinarmos o espectro original de $\nu_{x}$. 


\begin{tabular}{c}
\hline Parâmetros adotados como referência para uma SN \\
Distância para a SN : D = 10 kpc \\
Parametrização do espectro de acordo com a Eq. $(5.1)$, \\
sendo $\beta_{\alpha}=4$ todos os sabores \\
$\left\langle E_{\nu_{e}}\right\rangle=12 \mathrm{MeV} \quad\left\langle E_{\bar{\nu}_{e}}\right\rangle=15 \mathrm{MeV} \quad\left\langle E_{\nu_{x}}\right\rangle=18 \mathrm{MeV}$ \\
$E_{\nu_{\alpha}}^{\text {tot }}=\left\langle E_{\nu_{\alpha}}\right\rangle \Phi_{\nu_{\alpha}}=5 \times 10^{52}$ erg para todos os sabores \\
\hline \hline
\end{tabular}

Tabela 5.1: Parâmetros de referência de SN usados ao longo deste trabalho, a menos que explicitamente declarado diferente.

\begin{tabular}{|c|c|c|c|c|}
\hline & \multicolumn{3}{|c|}{ Composição Química dos Cintiladores } & \multirow[b]{2}{*}{ Suposições } \\
\hline Reação & $\begin{array}{c}\text { (a) } \mathrm{C}_{12} \mathrm{H}_{26}+\mathrm{C}_{9} \mathrm{H}_{12} \\
(80 \%+20 \%)\end{array}$ & $\begin{array}{c}\text { (b) } \mathrm{C}_{9} \mathrm{H}_{12} \\
\text { pseudocumeno }\end{array}$ & $\begin{array}{l}\text { (c) } \mathrm{C}_{6} \mathrm{H}_{5} \mathrm{C}_{12} \mathrm{H}_{25} \\
\text { alquilbenzeno }\end{array}$ & \\
\hline $\bar{\nu}_{e}+p \rightarrow n+e^{+}$ & 873 & 630 & 762 & Sem Oscilação \\
\hline $\bar{\nu}_{e}+p \rightarrow n+e^{+}$ & 924 & 669 & 804 & $\begin{array}{c}\bar{p}=c_{12}^{2}=0.69(\mathrm{NH}), \\
\left\langle E_{\nu_{x}}\right\rangle=18 \mathrm{MeV}\end{array}$ \\
\hline $\bar{\nu}_{e}+p \rightarrow n+e^{+}$ & 1038 & 750 & 903 & $\begin{array}{c}\bar{p}=0.0(\mathrm{IH}) \\
\left\langle E_{\nu_{x}}\right\rangle=18 \mathrm{MeV}\end{array}$ \\
\hline $\bar{\nu}_{e}+p \rightarrow n+e^{+}$ & 957 & 690 & 834 & $\begin{array}{c}\bar{p}=c_{12}^{2}=0.69(\mathrm{NH}), \\
\left\langle E_{\nu_{x}}\right\rangle=20 \mathrm{MeV}\end{array}$ \\
\hline $\bar{\nu}_{e}+p \rightarrow n+e^{+}$ & 1140 & 825 & 993 & $\begin{array}{c}\bar{p}=0.0(\mathrm{IH}) \\
\left\langle E_{\nu_{x}}\right\rangle=20 \mathrm{MeV}\end{array}$ \\
\hline $\bar{\nu}_{e}+p \rightarrow n+e^{+}$ & 987 & 714 & 858 & $\begin{array}{c}\bar{p}=c_{12}^{2}=0.69(\mathrm{NH}), \\
\left\langle E_{\nu_{x}}\right\rangle=22 \mathrm{MeV}\end{array}$ \\
\hline $\bar{\nu}_{e}+p \rightarrow n+e^{+}$ & 1239 & 894 & 1080 & $\begin{array}{c}\bar{p}=0.0(\mathrm{IH}) \\
\left\langle E_{\nu_{x}}\right\rangle=22 \mathrm{MeV}\end{array}$ \\
\hline$\nu+p \rightarrow \nu+p$ & 294 & 318 & 453 & $\begin{array}{c}\text { all flavors } T_{\text {que }}>0.2 \\
\mathrm{MeV},\left\langle E_{\nu_{x}}\right\rangle=18 \mathrm{MeV}\end{array}$ \\
\hline$\nu+p \rightarrow \nu+p$ & 399 & 405 & 561 & $\begin{array}{c}\text { all flavors } T_{\text {que }}>0.2 \\
\mathrm{MeV},\left\langle E_{\nu_{x}}\right\rangle=20 \mathrm{MeV}\end{array}$ \\
\hline$\nu+p \rightarrow \nu+p$ & 510 & 492 & 663 & $\begin{array}{c}\text { all flavors } T_{\text {que }}>0.2 \\
\mathrm{MeV},\left\langle E_{\nu_{x}}\right\rangle=22 \mathrm{MeV}\end{array}$ \\
\hline
\end{tabular}

Tabela 5.2: Número esperado de eventos de neutrinos de SN observados através do decaimento beta inverso e pelo espalhamento elástico próton-neutrino para os 3 tipos de líquidos cintiladores com três kt e uma SN distante $10 \mathrm{kpc}$ da Terra. Eles são (a) $80 \%$ de $\mathrm{C}_{12} \mathrm{H}_{26}$ e $20 \%$ de $\mathrm{C}_{9} \mathrm{H}_{12}$ usado em KamLAND, (b) $\mathrm{C}_{9} \mathrm{H}_{12}$ (pseudocumeno) usado no Borexino, e (c) $\mathrm{C}_{6} \mathrm{H}_{5} \mathrm{C}_{12} \mathrm{H}_{25}$ (alquilbenzeno) para ser usado no detector $\mathrm{SNO}+. \mathrm{NH}$ e IH indicam, respectivamente, hierarquias normal e invertida. Para $\nu+p \rightarrow \nu+p$ nós consideramos a energia cinética devido ao recuo do próton, $T_{\text {que }}$, com energia superior a $0,2 \mathrm{MeV}$, de acordo com as referências citadas no texto.

A tabela 5.1 contém os parâmetros adotados para uma SN e que foram usados ao longo deste trabalho.

Utilizando os resultados obtidos na Fig. 5.1 para o fluxo, calculamos o número de eventos que o detector de neutrinos de ANDES, com massa de 3 kton, registraria levando-se em conta os três tipos de líquido cintilador mencionados no cap. 4, o canal de reação, a hierarquia de massa (normal ou invertida) e se há ou não oscilação. Os resultados obtidos estão apresentados na tabela 5.2. Para o espalhamento $\nu+p \rightarrow \nu+p$ levamos em conta a energia cinética de recuo do próton e a perda de energia para o meio [131, 147]. 


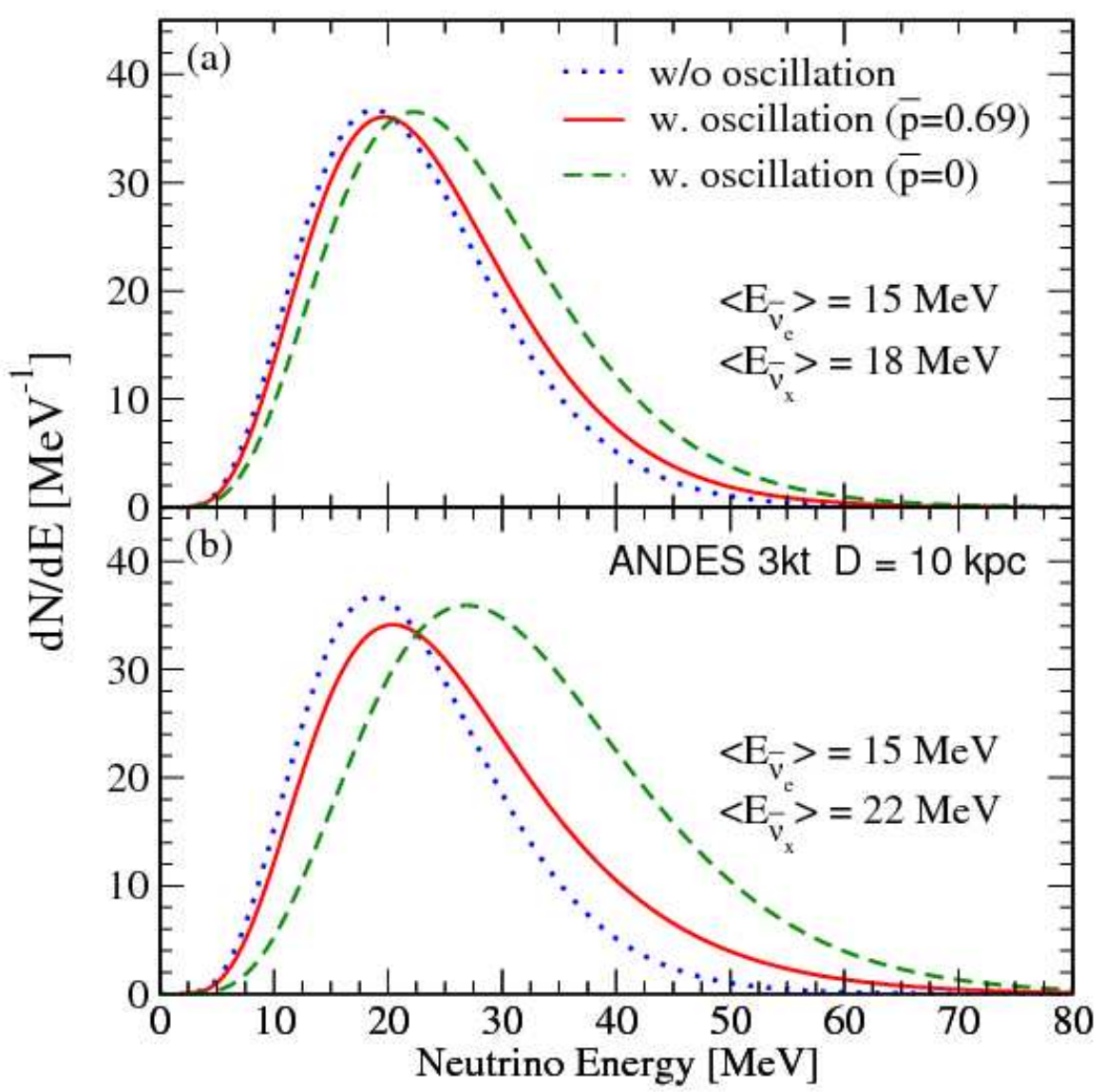

Figura 5.2: Distribuição de energia esperada de eventos de $\bar{\nu}_{e}$ para o detector de ANDES. Supomos $\left\langle E_{\bar{\nu}_{e}}\right\rangle=15 \mathrm{MeV}$ (curvas pontilhadas azuis) e $\left\langle E_{\bar{\nu}_{x}}\right\rangle=18 \mathrm{MeV}$ e 22 $\mathrm{MeV}$ para os gráficos superior e inferior, respectivamente (curvas verdes tracejadas).

\section{2}

\section{ANDES com Alquilbenzeno}

Nesta seção iremos considerar que o detector de neutrinos de ANDES usa o líquido cintilador 1,2,4-Alquilbenzeno, o mesmo que será empregado no detector $\mathrm{SNO}+[127]$.

Em relação as probabilidades de oscilação, iremos considerar os extremos de $\bar{p}$, isto é, $\bar{p}=0.0$ e $\bar{p}=0.69$ para atender as hierarquias invertida e normal, respectivamente. Outros efeitos, como turbulências e efeitos coletivos afetarão $\bar{p}$ de modo que seu valor ficará entre estes dois extremos. Assim, utilizando estes valores, calculamos a distribuição do número de eventos de energia esperado para antineutrinos eletrônicos vindos de uma SN distante $10 \mathrm{kpc}$ da Terra, $\frac{d N}{d E}$. Levamos em consideração os casos tanto da existência como da falta do fenômeno de oscilação de neutrinos. Os resultados são mostrados na Fig. 5.2. 
De acordo com a tabela 5.2 serão registrados entre 800 e 1000 eventos. Esta variação depende da hierarquia de massa e se há ou não oscilação. O efeito oscilatório faz com que a função $d N / d E$ seja deslocada para a direita no gráfico, uma vez que a seção de choque depende $\sim E_{\nu}^{2}$ que compensa a redução do fluxo devido as oscilações.

Como o espectro que chega à Terra é uma mistura dos espectros originais dos neutrinos, podemos tentar reconstruir o espectro original de luminosidade e energias médias de $\bar{\nu}_{e}$ e $\bar{\nu}_{x}$ baseando-nos nas detecções de $\bar{\nu}_{e}$ feitas pelos detectores terrestres [148, 149]. Entretanto, a possibilidade de haver degenerescências no parâmetros da SN e/ou a falta de registros de neutrinos de SN para reconhecermos como é uma SN padrão e, assim, reconhecer quando ocorrer uma explosão fora deste padrão, torna o processo de reconstrução em uma tarefa árdua [150, 151].

Entretanto, o uso de um cintilador permite que o detector de ANDES observe reações de corrente neutra tal como o espalhamento elástico neutrinopróton, $p+\nu \rightarrow p+\nu$, que é igual para todos os sabores. Assim, levando em conta a extinção de energia do próton via ionização, mostramos na Fig. 5.3 a distribuição do número de eventos esperados em função da energia de extinção considerando o detector de ANDES com massa de $3 \mathrm{kt}$ de cintilador e SN distante $10 \mathrm{kpc}$ da Terra, conforme a tabela 5.1. No nosso estudo consideramos apenas eventos onde $T_{\text {que }}>0.2 \mathrm{MeV}$ devido aos ruídos provenientes de decaimentos radioativos do próprio líquido cintilador e da vizinhança se tornarem mais intensos abaixo deste valor, assim como do decaimento beta do ${ }^{14} \mathrm{C}$.

Assim, com o baixo ruído para $T_{\text {que }}>0.2 \mathrm{MeV}$ e a possibilidade de mensurar o espectro de energia de extinção do recuo dos prótons pelo detector de ANDES, é possível reconstruir o fluxo de neutrinos $\left(d F_{\nu} / d E\right)$ em função da energia dos neutrinos onde $E_{\nu} \gtrsim 25 \mathrm{MeV}$, conforme mostrado em [131].

Na Fig. 5.4 mostramos o resultado para a reconstrução do fluxo de neutrinos com energia acima de $20 \mathrm{MeV}$ para uma SN ocorrida a $10 \mathrm{kpc}$ da Terra e detectada pelo detector de ANDES. A precisão na tentativa de reconstrução é de $15 \%$ para neutrinos com energia entre 20 e $40 \mathrm{MeV}$. A precisão esperada para o detector de ANDES no processo de reconstrução do fluxo de neutrinos é superior ao existente hoje nos detectores de tamanho similar, como o KamLAND e Borexino, ou no detector $\mathrm{SNO}+$, que entrará em operação em breve. 


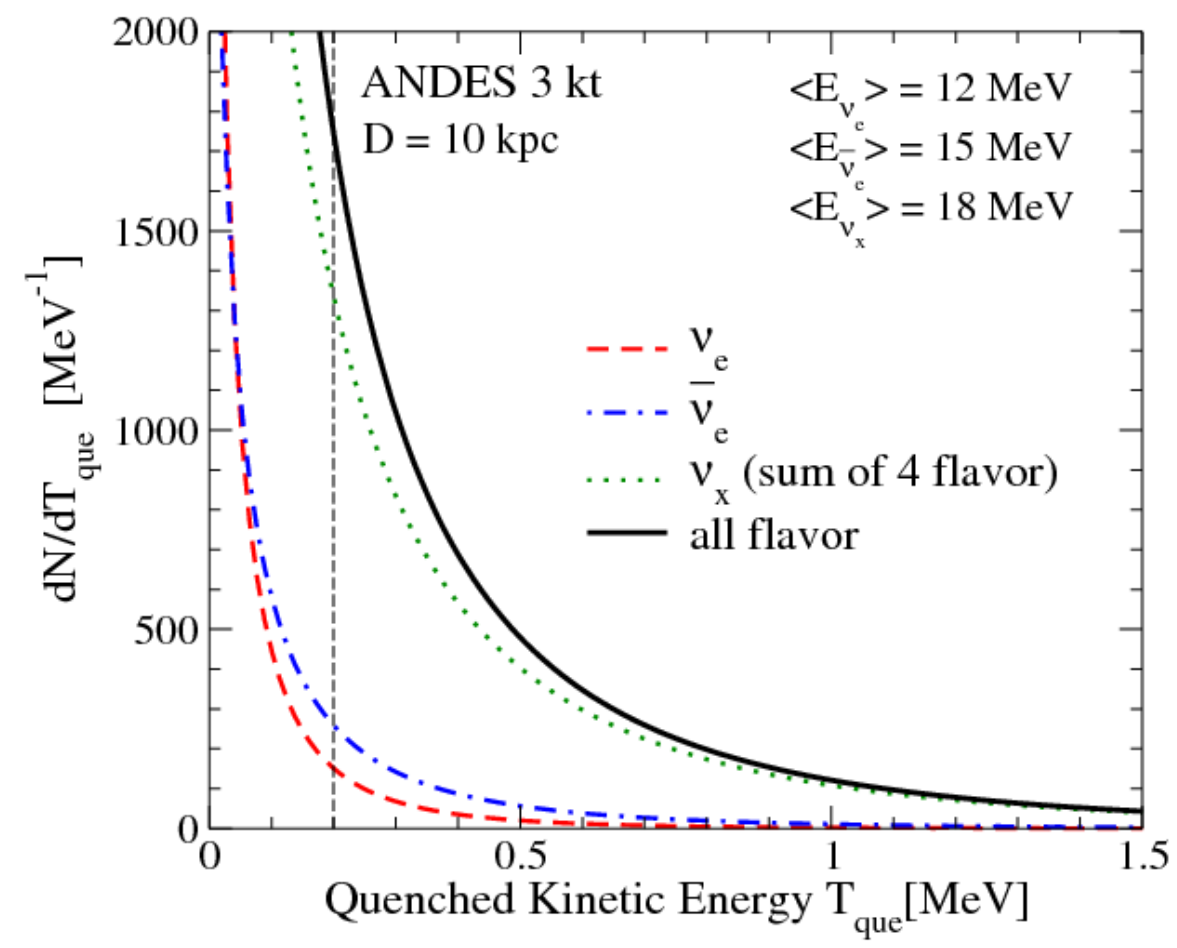

Figura 5.3: Distribuição de eventos em função da energia de extinção do próton para o detector de ANDES.

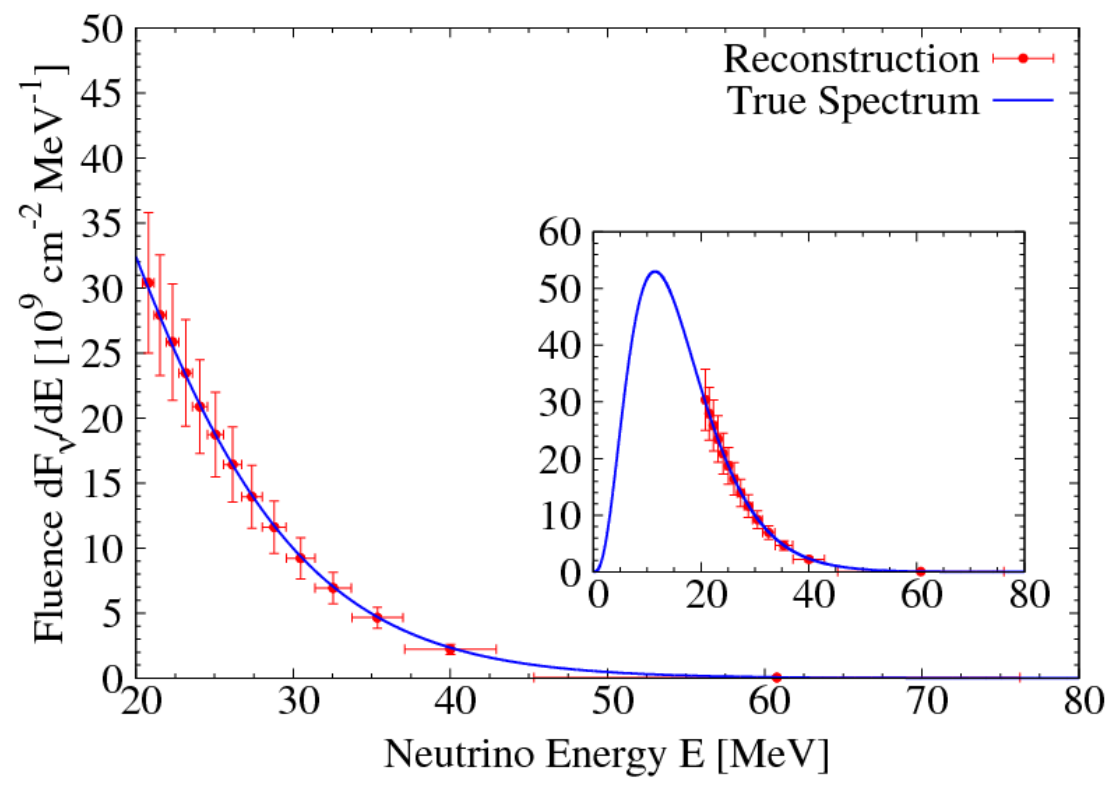

Figura 5.4: Os círculos vermelhos representam a reconstrução do fluxo original dos neutrinos provenientes de uma SN distante $10 \mathrm{kpc}$ da Terra para o detector de ANDES através do espalhamento elástico entre neutrino-próton. A barra de erro é equivalente a $1 \sigma$. 


\begin{tabular}{|l|c|c|c|}
\hline Nível de Confiança $\Rightarrow$ & $1 \sigma$ & $2 \sigma$ & $3 \sigma$ \\
\hline \hline$\left\langle E_{\nu_{x}}\right\rangle=15 \mathrm{MeV}$ & $8 \%$ & $13 \%$ & $18 \%$ \\
\hline$E_{\nu_{x}}^{\text {Tot }}=5 \times 10^{52} \mathrm{erg}$ & $30 \%$ & $53 \%$ & $>70 \%$ \\
\hline \hline$\left\langle E_{\nu_{x}}\right\rangle=18 \mathrm{MeV}$ & $6 \%$ & $9 \%$ & $13 \%$ \\
\hline$E_{\nu_{x}}^{\text {Tot }}=5 \times 10^{52} \mathrm{erg}$ & $17 \%$ & $28 \%$ & $40 \%$ \\
\hline \hline$\left\langle E_{\nu_{x}}\right\rangle=21 \mathrm{MeV}$ & $4 \%$ & $7 \%$ & $10 \%$ \\
\hline$E_{\nu_{x}}^{\text {Tot }}=5 \times 10^{52} \mathrm{erg}$ & $11 \%$ & $20 \%$ & $28 \%$ \\
\hline
\end{tabular}

Tabela 5.3: Precisão mostrada de acordo com nível de confiança para a determinação da $\left\langle E_{\nu_{x}}\right\rangle$ e da $E_{\nu_{x}}^{\text {Tot }}$ para o detector de ANDES devido à observação de uma SN conforme parâmetros da tabela 5.1. Os demais parâmetros foram mantido fixos. Na primeira coluna estão representados os valores de entrada.

Também estudamos com que precisão o detector de ANDES é capaz de determinar a energia média original $\left(\left\langle E_{\nu_{x}}\right\rangle\right)$ dos neutrinos/antineutrinos nãoeletrônicos $\left(\nu_{x}\right)$, i.e., a energia média de $\nu_{x}$ na neutrinosfera. Com este objetivo e utilizando os dados da nossa SN de referência dado pela tabela 5.1, analisamos o $\chi^{2}$, dado da forma

$$
\chi^{2}=\sum \frac{\left(N^{o b s}-N^{t e o}\right)^{2}}{N^{o b s}}
$$

considerando que os valores reais da energia média de $\nu_{x}$ e a energia total seriam $\left\langle E_{\nu_{x}}\right\rangle=15,18$ e $21 \mathrm{MeV}$ e $E_{\nu_{x}}^{\text {Tot }}=5 \times 10^{52} \mathrm{erg}$, respectivamente, onde $E_{\nu_{x}}^{T o t}$ é a energia total para um sabor de $\nu_{x}$ e o quádruplo deste valor equivale a energia total para todos os sabores de neutrinos não-eletrônicos. Os demais parâmetros foram mantido fixos para que pudéssemos perceber melhor o quão bem o detector de ANDES pode responder a este objetivo.

Assim, levando em conta apenas incertezas estatísticas, fizemos o gráfico de $E_{\nu_{x}}^{\text {Tot }}$ em função da $\left\langle E_{\nu_{x}}\right\rangle$ para os valores de 15,18 e $21 \mathrm{MeV}$. O resultado é mostrado na Fig. 5.5 onde podemos notar que a precisão torna-se melhor na medida que $\left\langle E_{\nu_{x}}\right\rangle$ afasta-se numericamente de $\left\langle E_{\bar{\nu}_{e}}\right\rangle$. A precisão com que o detector de ANDES pode determinar $\left\langle E_{\nu_{x}}\right\rangle$ e $E_{\nu_{x}}^{\text {Tot }}$ através da medida do espalhamento elástico neutrino-próton é mostrado na tabela 5.3.

\section{3}

\section{Comparando Eventos de Corrente Carregada e Corrente Neutra}

$\mathrm{Na}$ tentativa de verificar e mensurar - até certo ponto - o efeito de oscilação de neutrinos ou a probabilidade de sobrevivência $(\bar{p})$ dado pela Eq. 


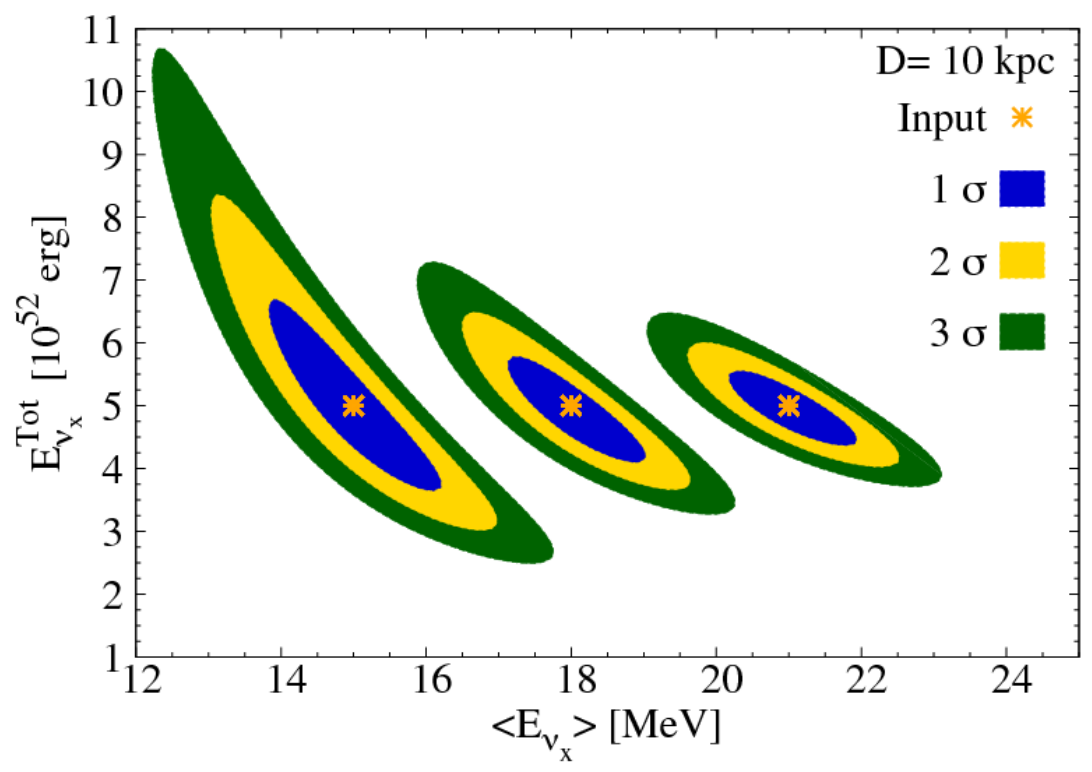

Figura 5.5: Sensitividade na determinação da $\left\langle E_{\nu_{x}}\right\rangle$ e da $E_{\nu_{x}}^{\text {Tot }}$ originais mostrados em 1, 2 e 3 níveis de confiança para o detector de ANDES através do número de eventos devido ao espalhamento elástico neutrino-próton. Consideramos 3 diferentes valores de entrada para $\left\langle E_{\nu_{x}}\right\rangle=15,18$ e $21 \mathrm{MeV}$ e a mesma $E_{\nu_{x}}^{T o t}=5 \times 10^{52} \mathrm{erg}$. Os valores de entrada estão indicados com um asterisco na figura.

(3.121) sem vincularmos o resultado a um modelo específico, comparamos os eventos de corrente carregada (CC) e os de corrente neutra $(\mathrm{CN})$, através da definição de

$$
R\left(N_{\bar{\nu}_{e} p} / N_{\nu p}\right) \equiv\left(\frac{N_{\bar{\nu}_{e} p}}{N_{\nu p}}\right)^{o b s}
$$

onde $\left(\frac{N_{\bar{\nu}_{e} p}}{N_{\nu p}}\right)^{o b s}$ é a razão entre o número de eventos de CC, via decaimento beta inverso, e de CN, via espalhamento elástico neutrino-próton. Note que isto é possível porque eventos de CN não dependem da oscilação de neutrinos, diferentemente dos eventos de CC.

Desta forma, podemos verificar qual é o comportamento de $R\left(N_{\bar{\nu}_{e} p} / N_{\nu p}\right)$ para diferentes valores de $\left\langle E_{\nu_{x}}\right\rangle$. Aqui, da mesma forma que fizemos anteriormente, os nossos resultados levam em conta apenas erros estatísticos. A Fig. 5.6 mostra o resultado para $\left\langle E_{\nu_{x}}\right\rangle$ no intervalo de 14 a $22 \mathrm{MeV}$ tanto para hierarquia normal $\bar{p}=0.69$ como para invertida $\bar{p}=0.0$ para o detector de ANDES. Na figura também testamos duas distâncias distintas para a SN: 5 e $10 \mathrm{kpc}$. Podemos observar que quanto maior for a diferença entre $\left\langle E_{\nu_{x}}\right\rangle$ e 


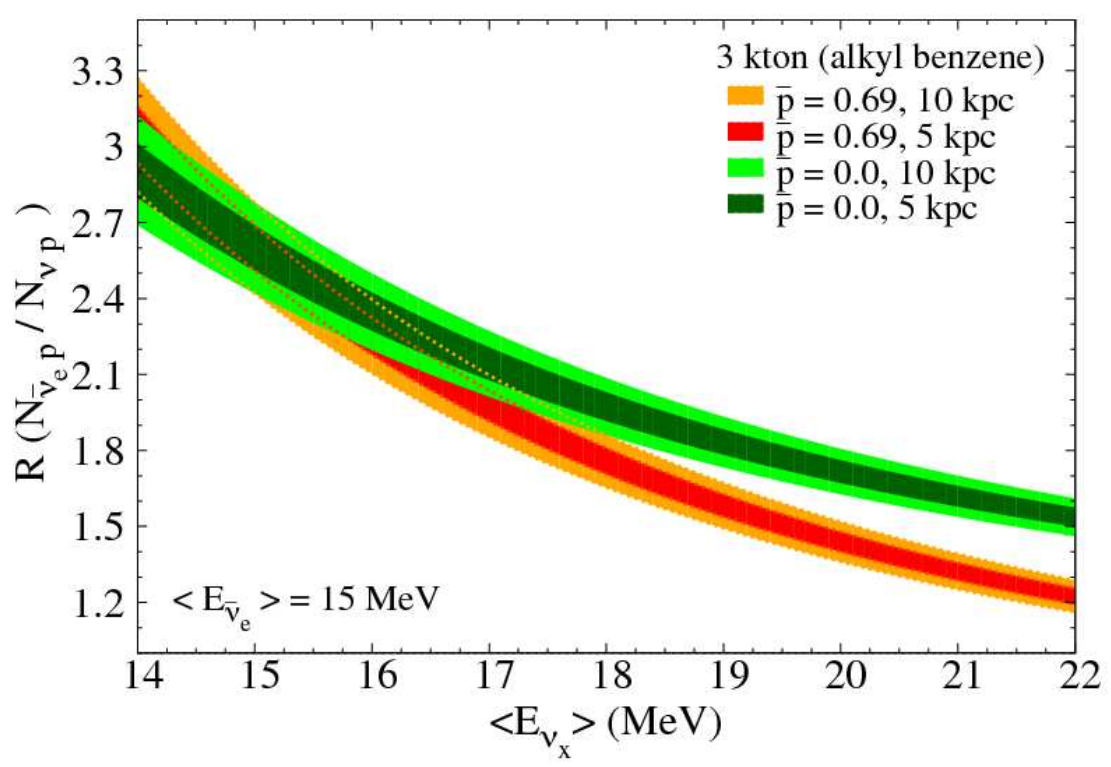

Figura 5.6: A razão $R\left(N_{\bar{\nu}_{e} p} / N_{\nu p}\right)$ em função de $\left\langle E_{\nu_{x}}\right\rangle$ para o detector de ANDES.

$\left\langle E_{\bar{\nu}_{e}}\right\rangle$, maior é a chance do detector de ANDES identificar a presença do efeito oscilatório pela dedução de $\bar{p}$. De acordo com recentes [152, 153, 154] simulações de explosão de SN, na fase de acreção podemos encontrar esta situação em que $\left\langle E_{\nu_{x}}\right\rangle$ e $\left\langle E_{\bar{\nu}_{e}}\right\rangle$ tornam-se significativamente diferentes. De fato, pode ocorrer que durante a fase de acreção os efeitos coletivos sejam fortemente suprimidos pela matéria e a conversão de sabores seja regida apenas pelo efeito MSW [145, 146]. Isto permitiria identificarmos os efeitos de oscilação mais facilmente, desde que tenhamos um número grande de eventos para esta fase.

Na Fig. 5.7 mantivemos $\left\langle E_{\nu_{x}}\right\rangle=18 \mathrm{MeV}$ constante e variamos $\left\langle E_{\bar{\nu}_{e}}\right\rangle$ para encontrar o seu valor verdadeiro. Novamente percebemos que quando $\left\langle E_{\bar{\nu}_{e}}\right\rangle$ aproxima-se de $\left\langle E_{\nu_{x}}\right\rangle$ torna-se difícil distinguir entre hierarquia normal $(\bar{p}=0.69)$ e hierarquia invertida $(\bar{p}=0.0)$. Se à época de ocorrência da próxima SN galáctica já conhecermos a hierarquia de massa dos neutrinos, podemos tentar encontrar os valores originais de $\left\langle E_{\nu_{x}}\right\rangle$ e $\left\langle E_{\bar{\nu}_{e}}\right\rangle$.

Estudando a razão entre o número de eventos de CC pelos de CN em função da probabilidade de sobrevivência, $0.0 \leq \bar{p} \leq 1.0$, e considerando $\left\langle E_{\nu_{x}}\right\rangle=18$ e $21 \mathrm{MeV}$ como os valores reais da $\left\langle E_{\nu_{x}}\right\rangle$, verificamos que o efeito de oscilação não pode ser distinguido a menos que $\left\langle E_{\nu_{x}}\right\rangle$ seja bastante diferente de $\left\langle E_{\bar{\nu}_{e}}\right\rangle$. Esta análise é mostrada na Fig. 5.8

Na Fig. 5.9 mostramos a razão do número de eventos $R\left(N_{\bar{\nu}_{e} p} / N_{\nu p}\right)$ em 


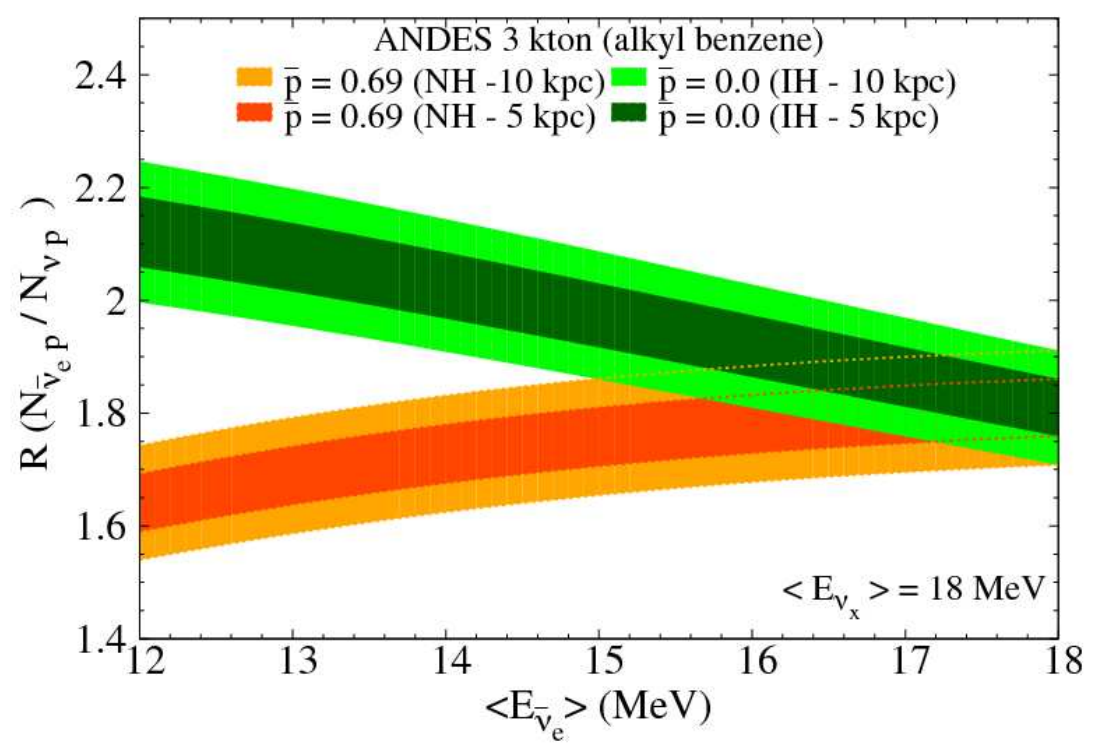

Figura 5.7: Agora razão $R\left(N_{\bar{\nu}_{e} p} / N_{\nu p}\right)$ em função de $\left\langle E_{\bar{\nu}_{e}}\right\rangle$ para o detector de ANDES.

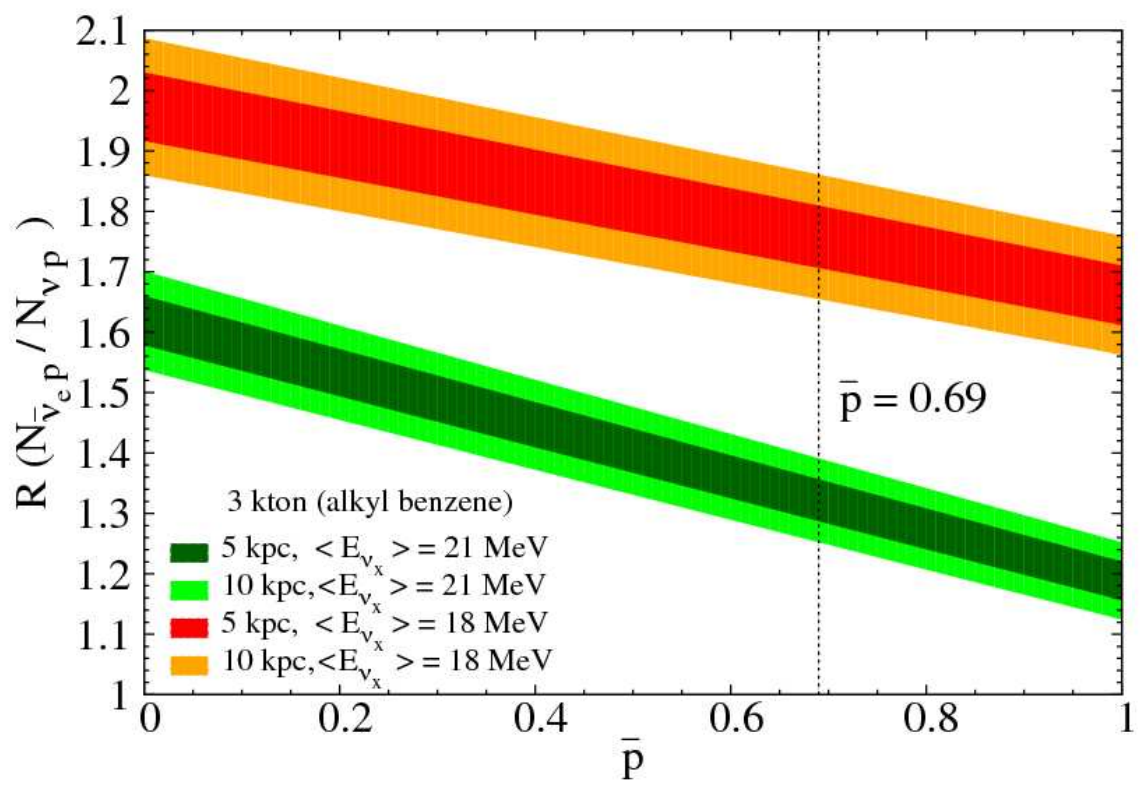

Figura 5.8: A razão $R\left(N_{\bar{\nu}_{e} p} / N_{\nu p}\right)$ em função de $\bar{p}$ para o detector de ANDES considerando $\left\langle E_{\nu_{x}}\right\rangle=18$ e $21 \mathrm{MeV}$.

função da razão das luminosidades $(L)$ de $\bar{\nu}_{e}$ e $\nu_{x}$, na forma

$$
\frac{L_{\bar{\nu}_{e}}}{L_{\nu_{x}}} \equiv \frac{\left(\left\langle E_{\bar{\nu}_{e}}\right\rangle \Phi_{\bar{\nu}_{e}}\right)}{\left(\left\langle E_{\nu_{x}}\right\rangle \Phi_{\nu_{x}}\right)}
$$




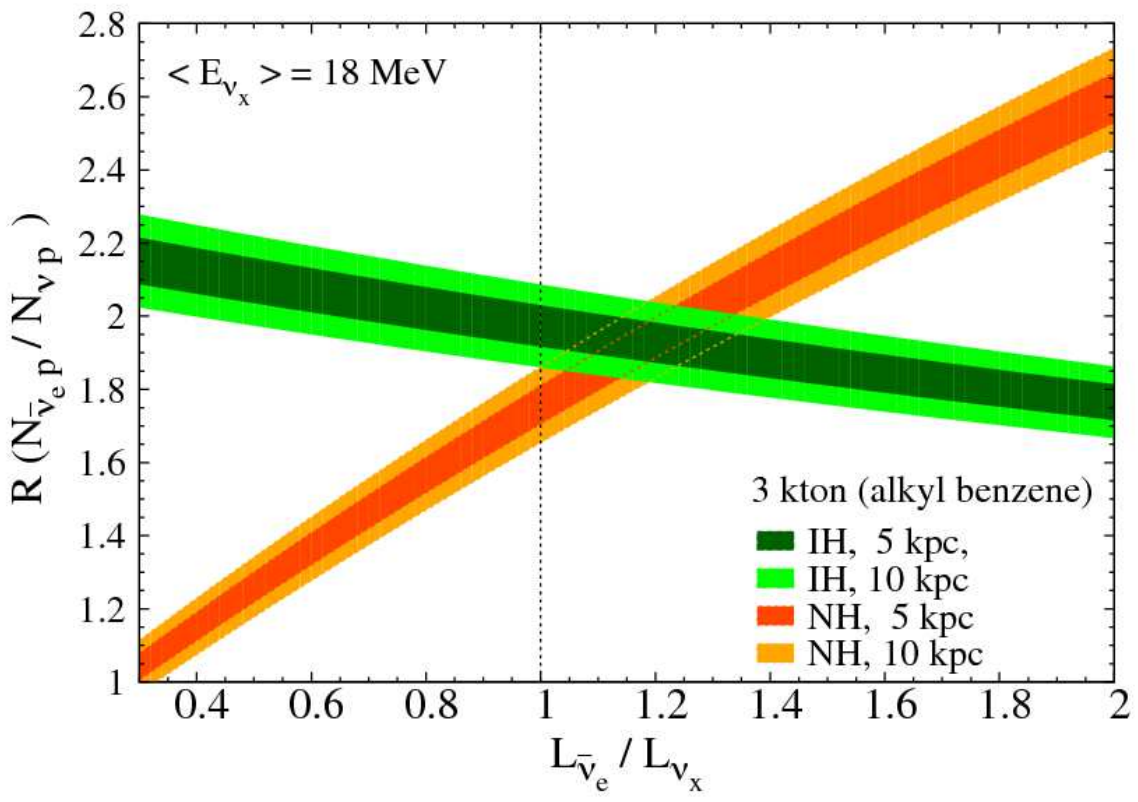

Figura 5.9: A razão $R\left(N_{\bar{\nu}_{e} p} / N_{\nu p}\right)$ em função da razão das luminosidades $L_{\bar{\nu}_{e}} / L_{\nu_{x}}$ para o caso onde $E_{\nu_{x}}=18 \mathrm{MeV}$ e os demais parâmetros são os mesmos da tabela 5.1.

Assim, o detector de ANDES pode distinguir a hierarquia de massa dos neutrinos se $L_{\bar{\nu}_{e}}$ for consideravelmente menor ou se for muito maior $(\sim 50 \%)$ que $L_{\nu_{x}}$. Se $L_{\bar{\nu}_{e}}$ for apenas $\sim 20 \%$ maior $L_{\nu_{x}}$, não é possível para o detector de ANDES fazer a distinção entre as hierarquias de massa. Se a hierarquia de massa já for conhecida quando da ocorrência da próxima SN galáctica, pode-se deduzir a diferença entre as luminosidades de $\bar{\nu}_{e}$ e $\nu_{x}$.

\section{4}

\section{Efeito de Matéria da Terra}

O detector de ANDES pode desempenhar papel importante na observação do efeito de matéria da Terra na detecção de neutrinos de uma SN Galáctica [22]. Através dele podemos determinar a hierarquia de massa dos neutrinos e propriedades do fluxo de neutrinos de SN [111, 155, 75, 156].

A ideia de utilizar mais de um detector de neutrinos para a observação do efeito de matéria da Terra consiste em comparar os resultados dos diversos detectores, sendo que em alguns a detecção é de neutrinos vindos diretamente da SN e em outros os neutrinos passam pelo interior da Terra. Estes últimos são os que sofrerão o efeito de matéria da Terra em comparação aos que chegaram diretamente da SN, sem cruzar o planeta. Obviamente, não podemos escolher qual detector receberá neutrinos diretamente da SN e qual receberá 
os neutrinos que cruzaram o interior terrestre. Isto depende do momento de chegada dos neutrinos e da posição da SN. Quando os neutrinos chegam ao detector após cruzar o planeta, dizemos que o detector foi "sombreado" pela Terra.

Apesar de existirem diversos locais onde estão instalados vários detectores de neutrinos ao redor do globo, nós utilizamos quatro para o nosso estudo de sombreamento. Dois no Hemisfério Norte e dois no Hemisfério Sul. São eles: Kamioka (Super-Kamiokande ou KamLAND), Polo Sul (IceCube), Sudbury $(\mathrm{SNO}+)$ e $A N D E S$.

Utilizando a distribuição de SN mostrada nas Fig. 2.9 e 2.10 e descrita nas eqs. (2.49) - (2.51) da seção 2.6 calculamos a probabilidade de sombreamento da Terra nos 4 detectores escolhidos para este trabalho. A última coluna da tabela 5.4 mostra o resultado considerando o sombreamento devido ao manto e ao núcleo terrestre, já que a densidade nestas duas regiões são distintas. Nesta tabela também estão indicadas as coordenadas geográficas dos nossos detectores de referência.

\begin{tabular}{|c|c|c|c|}
\hline Localização & Latitude & Longitude & $\begin{array}{c}\text { Prob. Sombreamento } \\
\text { Manto (Núcleo) }\end{array}$ \\
\hline Kamioka, Japão & $36.42^{\circ} \mathrm{N}$ & $137.3^{\circ} \mathrm{E}$ & $0.559(0.103)$ \\
Polo Sul & $90^{\circ} \mathrm{N}$ & - & $0.413(0.065)$ \\
ANDES & $30.25^{\circ} \mathrm{S}$ & $68.88^{\circ} \mathrm{W}$ & $0.449(0.067)$ \\
Sudbury, Canadá & $46.476^{\circ} \mathrm{N}$ & $81.20^{\circ} \mathrm{E}$ & $0.571(0.110)$ \\
\hline
\end{tabular}

Tabela 5.4: Coordenadas geográficas dos detectores considerados nesta tese. Na última coluna mostramos a probabilidade de neutrinos oriundos de uma SN galáctica cruzarem o interior da Terra, sendo através do Manto ou do conjunto Manto + Núcleo (indicado apenas como Núcleo).

O resultado para a probabilidade de sombreamento, tanto do Manto como do Núcleo, mostrado na tabela 5.4 é o caso mais simples, quando consideramos as probabilidades dos detectores individualmente. Podemos observar que os dois detectores localizados no Hemisfério Norte possuem uma maior chance de receber neutrinos que sofrem efeito de matéria da Terra em comparação aos localizados no Hemisfério Sul.

Em seguida, calculamos a probabilidade de sombreamento para um par de detectores, a saber, Kamioka e Polo Sul, para comparar com a Ref. [80]. Nesta situação, há 4 diferentes possibilidades: 1) ambos são sombreados pela Terra; 2) um é sombreado e o outro não; 3) oposto a situação $\underline{2}$; e 4) nenhum dos 
dois locais é sombreado pela Terra. Quando há o sombreamento, escrevemos "sim" e em caso negativo, "não". Os resultados são mostrados na tabela 5.5 e estão em concordância com o obtido em [80]. Podemos observar que temos uma probabilidade de $\sim 72 \%$ de que neutrinos provenientes de uma SN galáctica e tenham cruzado o interior do planeta sejam observados por somente um dos detectores. Para que os neutrinos cruzem o planeta pela região central, i.e., pelo núcleo, a probabilidade é de $\sim 17 \%$.

\begin{tabular}{|c|c|c|c|}
\hline & \multicolumn{2}{|c|}{ Efeito de Matéria da Terra } & \\
\hline Caso & Kamioka & Polo Sul & $\begin{array}{c}\text { Prob. Sombreamento } \\
\text { Manto (Núcleo) }\end{array}$ \\
\hline$(1)$ & Não & Não & $0.152(0.832)$ \\
$(2)$ & Sim & Não & $0.435(0.104)$ \\
$(3)$ & Não & Sim & $0.288(0.065)$ \\
$(4)$ & Sim & Sim & $0.125(0.000)$ \\
\hline
\end{tabular}

Tabela 5.5: Probabilidade de sombreamento da Terra para a associação de dois detectores localizados em Kamioka, no Japão, e no Polo Sul.

\begin{tabular}{|c|c|c|c|c|}
\hline & \multicolumn{2}{|c|}{ Efeito de Matéria da Terra } & \\
\hline Caso & Kamioka & Polo Sul & ANDES & $\begin{array}{c}\text { Prob. Sombreamento } \\
\text { Manto (Núcleo) }\end{array}$ \\
\hline$(1)$ & Não & Não & Não & $0.024(0.767)$ \\
\hline$(2)$ & Sim & Não & Não & $0.388(0.105)$ \\
$(3)$ & Não & Sim & Não & $0.034(0.061)$ \\
$(4)$ & Não & Não & Sim & $0.128(0.063)$ \\
\hline$(5)$ & Sim & Sim & Não & $0.106(0.000)$ \\
$(6)$ & Não & Sim & Sim & $0.254(0.003)$ \\
$(7)$ & Sim & Não & Sim & $0.047(0.000)$ \\
\hline$(8)$ & Sim & Sim & Sim & $0.020(0.000)$ \\
\hline
\end{tabular}

Tabela 5.6: Probabilidade de sombreamento pela Terra para o caso onde temos três detectores combinados: Super-Kamiokande, IceCube e ANDES.

Expandindo para três detectores, incluímos o detector de ANDES, objeto do nosso estudo. Neste caso, temos um total de 8 combinações possíveis que estão mostrados na tabela 5.6. Agora, podemos ver que a probabilidade de observar neutrinos que atravessam a Terra e são detectados por pelo menos 1 detector enquanto que ao menos um dos outros detectores recebam neutrinos diretamente da $\mathrm{SN}$, sobe para $\sim 96 \%$. Isso é um aumento de $30 \%$ sobre o valor para dois detectores (Kamioka e Polo Sul). Na verdade, a comparação 


\begin{tabular}{|c|c|c|c|c|c|}
\hline & \multicolumn{3}{|c|}{ Efeito de Matéria da Terra } & \\
\hline Caso & Kamioka & Polo Sul & ANDES & Sudbury & $\begin{array}{c}\text { Prob. Sombreamento } \\
\text { Manto (Núcleo) }\end{array}$ \\
\hline$(1)$ & Não & Não & Não & Não & $0.008(0.657)$ \\
\hline$(2)$ & Sim & Não & Não & Não & $0.206(0.105)$ \\
$(3)$ & Não & Sim & Não & Não & $0.034(0.061)$ \\
$(4)$ & Não & Não & Sim & Não & $0.001(0.063)$ \\
$(5)$ & Não & Não & Não & Sim & $0.016(0.111)$ \\
\hline$(6)$ & Sim & Sim & Não & Não & $0.205(0.000)$ \\
$(7)$ & Sim & Não & Sim & Não & $0.000(0.000)$ \\
$(8)$ & Sim & Não & Não & Sim & $0.282(0.000)$ \\
$(9)$ & Não & Sim & Sim & Não & $0.163(0.003)$ \\
$(10)$ & Não & Sim & Não & Sim & $0.000(0.000)$ \\
$(11)$ & Não & Não & Sim & Sim & $0.127(0.000)$ \\
\hline$(12)$ & Não & Sim & Sim & Sim & $0.091(0.000)$ \\
$(13)$ & Sim & Não & Sim & Sim & $0.047(0.000)$ \\
$(14)$ & Sim & Sim & Não & Sim & $0.011(0.000)$ \\
$(15)$ & Sim & Sim & Sim & Não & $0.012(0.000)$ \\
\hline$(16)$ & Sim & Sim & Sim & Sim & $0.008(0.000)$ \\
\hline
\end{tabular}

Tabela 5.7: Probabilidade de sombreamento da Terra para o caso com quatro detectores: Kamioka, Polo Sul, ANDES e Sudbury.

com qualquer combinação de dois detectores feita na Ref. [80], terá resultado favorável à combinação tripla. Naquele estudo, o melhor conjunto foi Pyhäsalmi e Polo Sul, com probabilidade de sombreamento de $\sim 87 \%$. A possibilidade de que os neutrinos cruzem o núcleo da Terra, nesta situação, é mensurada em $23 \%$.

Finalmente, fizemos a análise para a combinação de quatro detectores localizados em Kamioka, Polo Sul, Andes e Sudbury. Nesta nova configuração, temos 16 combinações possíveis e a probabilidade de que pelo menos um dos detectores observe neutrinos vindos através da Terra e, ao menos um dos outros três detectores registre neutrinos vindos diretamente da SN, sobe para 98\%. Também encontramos a probabilidade de $\sim 34 \%$ para que em pelo menos um destes locais receba neutrinos que cruzaram o núcleo da Terra, o que significa um aumento de $\sim 68 \%$ quando comparado com o caso de três detectores. Nossos resultados estão explicitados na tabela 5.7.

Na tentativa de quantificarmos o efeito de matéria da Terra como função da energia do neutrino e do ângulo de incidência da SN, mostramos na Fig. 5.10, os isocontornos de $P^{\oplus}\left(\bar{\nu}_{1} \rightarrow \bar{\nu}_{e}\right) / c_{12}^{2}$ no plano do ângulo nadiral $\left(\theta_{\text {nadir }}\right)$ 


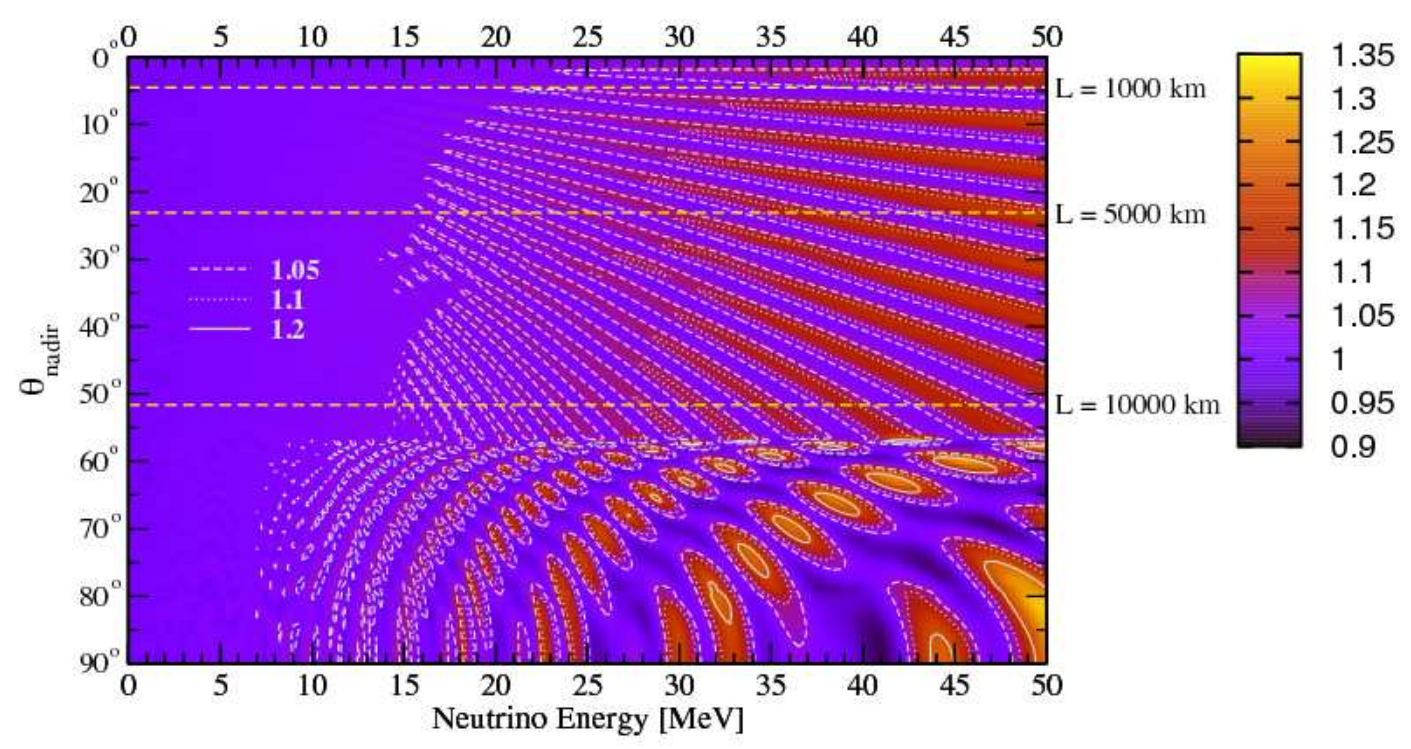

Figura 5.10: Um oscilograma de neutrino onde o isocontorno da razão $P^{\oplus}\left(\bar{\nu}_{1} \rightarrow\right.$ $\left.\bar{\nu}_{e}\right) / c_{12}^{2}$ é feito no plano do ângulo nadiral, $\theta_{\text {nadir }}$, e da energia do neutrino. O valor unitário no gráfico representa a inexistência (ausência) do efeito de matéria da Terra.

e a energia do neutrino, onde $P^{\oplus}\left(\bar{\nu}_{1} \rightarrow \bar{\nu}_{e}\right)$ foi obtido por integração numérica da equação de evolução dos neutrinos usando o perfil de densidade de matéria terrestre baseado no Modelo Preliminar de Referência da Terra (PREM, em inglês) [157]. Importante observar que qualquer valor diferente de um na quantidade $P^{\oplus}\left(\bar{\nu}_{1} \rightarrow \bar{\nu}_{e}\right)$ implica na presença de efeito de matéria da Terra.

Na Fig. 5.10 o ângulo nadiral $\left(\theta_{\text {nadir }}\right)$ está definido como $\theta_{\text {nadir }}=90^{\circ}$ para os neutrinos que alcançam o detector cruzando a Terra, i.e., é o oposto angular do zênite. $\mathrm{O}$ valor $\theta_{\text {nadir }}=0^{\circ}$ equivale aos neutrinos que vem da direção horizontal. Assim, obviamente, $\theta_{\text {nadir }}$ é uma medida local do detector e variará de um local para outro na análise de uma mesma SN. Ainda desta figura, podemos observar que o efeito de matéria da Terra é mais evidente (forte) quando os neutrinos cruzam o núcleo terrestre $\left(\theta_{\text {nadir }} \gtrsim 57^{\circ}\right)$ e com maior energia. Este gráfico é similar ao mostrado nas Refs. [158, 159] usado para o estudo de neutrinos atmosféricos e foi chamado de "oscilograma de neutrino".

Depois, com os valores dados pela eq. 3.144 podemos analisar a diferença fracional do fluxo quando há ou não efeito de matéria da Terra. Fizemos $\Delta F_{\bar{\nu}_{e}} / F_{\bar{\nu}_{e}}$ para três diferentes percursos no interior terrestre: $L=1000 \mathrm{~km}$ (linha contínua), $L=5000 \mathrm{~km}$ (linha pontilhada) e $L=10000 \mathrm{~km}$ (linha tracejada). Pode-se observar que o efeito de matéria da Terra é mais forte para neutrinos de maior energia. Porém, a quantidade de eventos é inversamente 


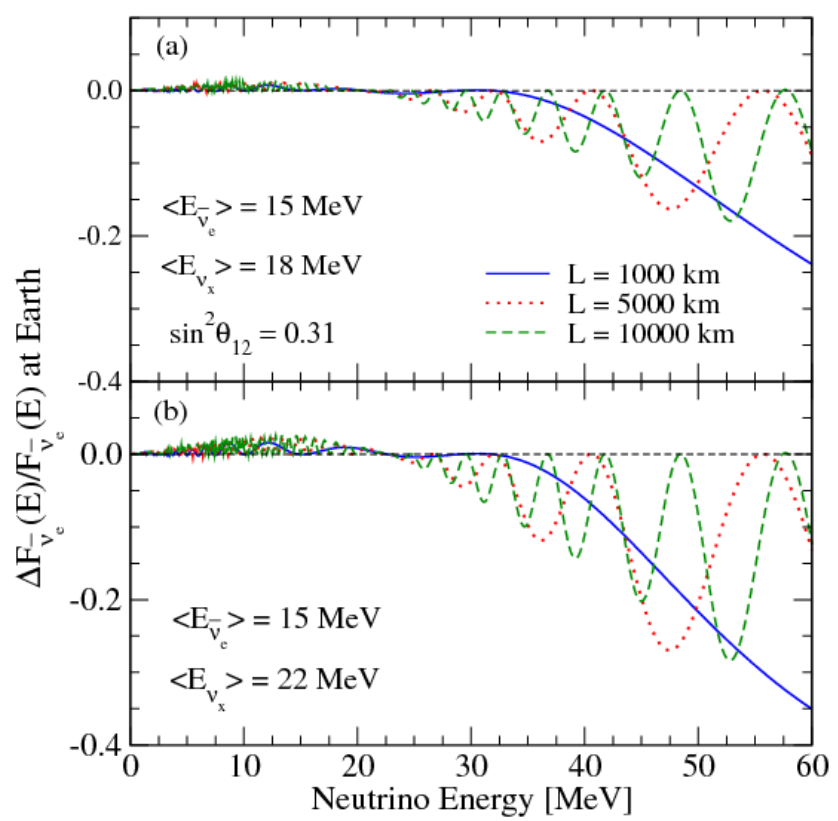

Figura 5.11: Diferença fracional no espectro do fluxo de neutrinos com e sem o efeito de matéria da Terra, $\Delta F_{\bar{\nu}_{e}} / F_{\bar{\nu}_{e}}$.

proporcional a energia, i.e., quanto maior a energia, menor será o número de eventos. Assim, para observar o efeito de matéria, tanto ele próprio como o número de eventos na faixa de energia adequada precisam ser grandes o suficiente.

Devido a impossibilidade de compararmos, no mesmo detector, o número de eventos com e sem o efeito de matéria da Terra, precisamos utilizar outro detector. Assim, um detector registrará eventos vindo diretamente da SN e o outro receberá os neutrinos que cruzaram o interior terrestre antes de chegarem ao detector, sofrendo deste jeito, o efeito de matéria. Os detectores Super-Kamiokande e ANDES podem fazer um bom par. Consideramos que o laboratório situado no Hemisfério Sul será sombreado enquanto que o do Hemisfério Norte não. Então, dentro de erros estatísticos e sistemáticos usuais, pode-se inferir o fluxo de neutrinos de uma SN galáctica no SK a partir dos resultados do detector de ANDES, como se o SK também tivesse sido sombreado pela Terra. Evidentemente, o oposto também pode ser executado. Isso é válido porque se ambos os detectores não sofrerem sombreamento da Terra, em teoria, devem registrar o mesmo espectro. Se há o efeito de matéria, os espectros devem diferir entre si, digo, não deve haver coincidência de espectros. 
Com a combinação de ANDES e Super-Kamiokande é possível distinguir a distribuição de eventos devido ao vácuo e à matéria. Na tabela 5.8 estão os resultados para nosso estudo para o número esperado de eventos no SuperKamiokande para quatro distintas faixas de energia considerando vácuo e matéria, onde o percurso no interior da Terra seja $L=1000 \mathrm{~km}$ e diferentes parâmetros de SN. Conjecturando que o espectro de distribuição de eventos de neutrinos, sombreados pela Terra, que chega no Super-Kamiokande seja fornecido pelo detector de ANDES com uma incerteza estatística para cada faixa de energia equivalente ao do detector sul-americano, podemos estimar em qual dos casos está presente o efeito de matéria

De acordo com a Fig. 5.11 não há sensibilidade para detectar efeito de matéria da Terra na primeira faixa de energia $(E<30 \mathrm{MeV})$, então podemos utilizá-la como faixa controladora para normalizar a distribuição fornecida pelos dados do detector de ANDES que tem efeito de matéria. Devido a necessidade de um grande número de eventos no detector de ANDES, consideramos que a SN ocorre a uma distância de 5 kpc da Terra.

Finalmente, testamos a hipótese para a distribuição no vácuo para cada modelo de SN listado na sexta coluna da tabela 5.8 com o intuito de ter uma ideia quantitativa do poder de discriminação entre as duas possibilidades. Comparamos o número de eventos no SK com e sem o efeito de matéria onde para a primeira situação (com) fizemos a extrapolação do número de eventos do detector de ANDES, que conjecturamos receber os neutrinos após estes viajarem $1000 \mathrm{~km}$ pelo interior da Terra. Utilizamos as duas últimas faixas de energia para estimar o desvio do vácuo em termos dos desvios padrões e os resultados são mostrados na última coluna da tabela 5.8. Na maioria dos casos, o efeito de matéria pode ser identificado com mais de $2 \sigma$. Outrossim, testamos que se $\left\langle E_{\nu_{x}}\right\rangle \lesssim 18 \mathrm{MeV}$ não podemos distinguir o efeito de matéria para qualquer valor dos parâmetros utilizados na tabela 5.8, ao menos para $L_{\overline{\nu_{e}}} / L_{\nu_{x}}=1$.

\section{5}

\section{Triangulação}

Nesta parte do trabalho levamos em conta a distribuição de SN mostrada na seção 2.6 e estudamos a contribuição do laboratório de ANDES para determinar a localização de uma SN galáctica utilizando apenas neutrinos, uma vez que eles chegarão na Terra antes dos fótons. A importância desta ação é 


\begin{tabular}{|c|c|c|c|c|c|c|}
\hline & $E<30 \mathrm{MeV}$ & $30<E / \mathrm{MeV}<40$ & $40<E / \mathrm{MeV}<50$ & $E>50 \mathrm{MeV}$ & Caso & Comp. \\
\hline $\begin{array}{c}\text { Vácuo (o) } \\
1000 \mathrm{~km}(\mathrm{p})\end{array}$ & $\begin{array}{l}18159 \pm 135 \\
18132 \pm 374\end{array}$ & $\begin{array}{l}4973 \pm 71 \\
5065 \pm 198\end{array}$ & $\begin{array}{l}2032 \pm 45 \\
1908 \pm 121\end{array}$ & $\begin{array}{l}889 \pm 30 \\
700 \pm 74\end{array}$ & $\begin{array}{c}\left\langle E_{\nu_{x}}\right\rangle=22 \mathrm{MeV} \\
\beta_{x}=\beta_{e}=4\end{array}$ & $3.1 \sigma$ \\
\hline $\begin{array}{c}\text { Vácuo (o) } \\
1000 \mathrm{~km}(\mathrm{p})\end{array}$ & $\begin{array}{l}17395 \pm 132 \\
17370 \pm 367\end{array}$ & $\begin{array}{l}5785 \pm 76 \\
5858 \pm 213\end{array}$ & $\begin{array}{l}2583 \pm 51 \\
2483 \pm 139\end{array}$ & $\begin{array}{r}1147 \pm 34 \\
988 \pm 87\end{array}$ & $\begin{array}{c}\left\langle E_{\nu_{x}}\right\rangle=22 \mathrm{MeV} \\
\beta_{x}=4 \beta_{e}=3\end{array}$ & $2.2 \sigma$ \\
\hline $\begin{array}{c}\text { Vácuo (o) } \\
1000 \mathrm{~km}(\mathrm{p})\end{array}$ & $\begin{array}{l}16031 \pm 127 \\
16011 \pm 352\end{array}$ & $\begin{array}{l}6674 \pm 82 \\
6728 \pm 228\end{array}$ & $\begin{array}{l}3594 \pm 60 \\
3541 \pm 166\end{array}$ & $\begin{array}{l}1978 \pm 45 \\
1917 \pm 122\end{array}$ & $\begin{array}{c}\left\langle E_{\nu_{x}}\right\rangle=22 \mathrm{MeV} \\
\beta_{x}=4 \beta_{e}=2\end{array}$ & $0.7 \sigma$ \\
\hline $\begin{array}{l}\text { Vácuo (o) } \\
1000 \mathrm{~km}(\mathrm{p})\end{array}$ & $\begin{array}{l}16863 \pm 130 \\
16837 \pm 361\end{array}$ & $\begin{array}{l}5722 \pm 76 \\
5787 \pm 212\end{array}$ & $\begin{array}{l}2864 \pm 54 \\
2731 \pm 145\end{array}$ & $\begin{array}{l}1604 \pm 40 \\
1321 \pm 101\end{array}$ & $\begin{array}{c}\left\langle E_{\nu_{x}}\right\rangle=22 \mathrm{MeV} \\
\beta_{x}=\beta_{e}=3\end{array}$ & $3.2 \sigma$ \\
\hline $\begin{array}{l}\text { Vácuo (o) } \\
1000 \mathrm{~km} \mathrm{(p)}\end{array}$ & $\begin{array}{l}15499 \pm 125 \\
15479 \pm 346\end{array}$ & $\begin{array}{l}6611 \pm 81 \\
6657 \pm 227\end{array}$ & $\begin{array}{l}3875 \pm 62 \\
3789 \pm 171\end{array}$ & $\begin{array}{l}2434 \pm 49 \\
2250 \pm 132\end{array}$ & $\begin{array}{c}\left\langle E_{\nu_{x}}\right\rangle=22 \mathrm{MeV} \\
\beta_{x}=3 \beta_{e}=2\end{array}$ & $1.7 \sigma$ \\
\hline $\begin{array}{c}\text { Vácuo (o) } \\
1000 \mathrm{~km} \mathrm{(p)}\end{array}$ & $\begin{array}{l}14790 \pm 122 \\
14766 \pm 338\end{array}$ & $\begin{array}{l}6388 \pm 80 \\
6419 \pm 223\end{array}$ & $\begin{array}{l}4089 \pm 64 \\
3971 \pm 175\end{array}$ & $\begin{array}{l}3059 \pm 55 \\
2701 \pm 145\end{array}$ & $\begin{array}{c}\left\langle E_{\nu_{x}}\right\rangle=22 \mathrm{MeV} \\
\beta_{x}=\beta_{e}=2\end{array}$ & $2.8 \sigma$ \\
\hline 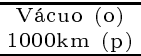 & $\begin{array}{l}17686 \pm 133 \\
17655 \pm 370\end{array}$ & $\begin{array}{l}5240 \pm 72 \\
5343 \pm 203\end{array}$ & $\begin{array}{l}2439 \pm 49 \\
2272 \pm 133\end{array}$ & $\begin{array}{c}1285 \pm 36 \\
990 \pm 88\end{array}$ & $\begin{array}{c}\left\langle E_{\nu_{x}}\right\rangle=24 \mathrm{MeV} \\
\beta_{x}=\beta_{e}=4\end{array}$ & $4.3 \sigma$ \\
\hline $\begin{array}{l}\text { Vácuo (o) } \\
1000 \mathrm{~km}(\mathrm{p})\end{array}$ & $\begin{array}{l}16922 \pm 130 \\
16892 \pm 362\end{array}$ & $\begin{array}{l}6052 \pm 78 \\
6136 \pm 218\end{array}$ & $\begin{array}{l}2990 \pm 55 \\
2847 \pm 148\end{array}$ & $\begin{array}{l}1543 \pm 39 \\
1278 \pm 100\end{array}$ & $\begin{array}{c}\left\langle E_{\nu_{x}}\right\rangle=24 \mathrm{MeV} \\
\beta_{x}=4 \beta_{e}=3\end{array}$ & $3.1 \sigma$ \\
\hline $\begin{array}{l}\text { Vácuo (o) } \\
1000 \mathrm{~km}(\mathrm{p})\end{array}$ & $\begin{array}{l}15557 \pm 125 \\
15533 \pm 347\end{array}$ & $\begin{array}{l}6941 \pm 83 \\
7006 \pm 233\end{array}$ & $\begin{array}{l}4001 \pm 63 \\
3905 \pm 174\end{array}$ & $\begin{array}{l}2374 \pm 49 \\
2207 \pm 131\end{array}$ & $\begin{array}{c}\left\langle E_{\nu_{x}}\right\rangle=24 \mathrm{MeV} \\
\beta_{x}=4 \beta_{e}=2\end{array}$ & $1.7 \sigma$ \\
\hline $\begin{array}{c}\text { Vácuo(o) } \\
1000 \mathrm{~km}(\mathrm{p})\end{array}$ & $\begin{array}{l}16441 \pm 128 \\
16409 \pm 356\end{array}$ & $\begin{array}{l}5858 \pm 77 \\
5928 \pm 214\end{array}$ & $\begin{array}{l}3174 \pm 56 \\
3007 \pm 153\end{array}$ & $\begin{array}{l}2022 \pm 45 \\
1625 \pm 112\end{array}$ & $\begin{array}{c}\left\langle E_{\nu_{x}}\right\rangle=24 \mathrm{MeV} \\
\beta_{x}=\beta_{e}=3\end{array}$ & $4.0 \sigma$ \\
\hline 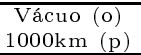 & $\begin{array}{l}15077 \pm 123 \\
15051 \pm 341\end{array}$ & $\begin{array}{l}6746 \pm 82 \\
6797 \pm 229 \\
\end{array}$ & $\begin{array}{l}4185 \pm 65 \\
4065 \pm 177\end{array}$ & $\begin{array}{l}2853 \pm 53 \\
2554 \pm 141 \\
\end{array}$ & $\begin{array}{c}\left\langle E_{\nu_{x}}\right\rangle=24 \mathrm{MeV} \\
\beta_{x}=3 \beta_{e}=2\end{array}$ & $2.5 \sigma$ \\
\hline 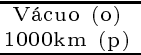 & $\begin{array}{l}14439 \pm 120 \\
14410 \pm 334\end{array}$ & $\begin{array}{l}6400 \pm 80 \\
6430 \pm 223\end{array}$ & $\begin{array}{l}4248 \pm 65 \\
4116 \pm 179\end{array}$ & $\begin{array}{l}3402 \pm 58 \\
2948 \pm 151\end{array}$ & $\begin{array}{c}\left\langle E_{\nu_{x}}\right\rangle=24 \mathrm{MeV} \\
\beta_{x}=\beta_{e}=2\end{array}$ & $3.3 \sigma$ \\
\hline
\end{tabular}

Tabela 5.8: Número de eventos esperados de decaimento $\beta$ inverso no detector de Super-Kamiokande $\left(1.7 \times 10^{33}\right.$ prótons livres $)$ para uma SN distante $5 \mathrm{kpc}$ da Terra para $E<30 \mathrm{MeV}, 30<E / \mathrm{MeV}<40,40<E / \mathrm{MeV}<50$ e $E>50 \mathrm{MeV}$ para o caso de vácuo e efeito de matéria com uma distância de $1000 \mathrm{~km}$ e diversos parâmetros de SN. Nós supomos $L_{\overline{\nu_{e}}} / L_{\nu_{x}}=1$ and $\left\langle E_{\bar{\nu}_{e}}\right\rangle=15 \mathrm{MeV}$, assim como que o detector de Super-Kamiokande recebe neutrinos sem o efeito de matéria da Terra enquanto que o detector de ANDES recebe os neutrinos após os mesmos terem viajado $1000 \mathrm{~km}$ dentro do planeta. Os números na linha indicada como "vácuo" são aqueles a serem observados pelo detector SK, que precisa compará-los com a previsão teórica para o próprio SK, calculada do número de eventos observados no detector de ANDES. Na última coluna nós mostramos em quantos $\sigma$ a observação em $1000 \mathrm{~km}$ é distinguível do vácuo, e Comp. é a abreviação para "Compatibilidade". Na primeira coluna, ㅇ significa "observado" e $\underline{\mathrm{p}}$, "previsto".

permitir que a comunidade astronômica registre a chegada dos primeiros fótons ao planeta, i.e., o início da curva de luz do espectro da SN.

Assim, seja um detector qualquer cujo vetor posição é dado por

$$
\mathbf{x}_{i}=\left(x_{i}, y_{i}, z_{i}\right)
$$

para relacionar a posição do detector com a posição da SN, utilizamos o sistema de coordenadas equatoriais, onde a Terra está localizada na origem e o Polo Norte terrestre coincide com o polo norte equatorial. Neste sistema, as coordenadas utilizadas são a ascensão reta $(\alpha)$ e a declinação $(\delta)$ e as unidades são expressadas em hora sideral e graus, respectivamente. O vetor posição da eq. 5.7 pode ser reescrito em função das coordenadas equatoriais da seguinte 
forma:

$$
\begin{aligned}
x_{i}(t) & =R_{\oplus} \cos \phi_{i}(t) \sin \theta_{i} \\
y_{i}(t) & =R_{\oplus} \sin \phi_{i}(t) \sin \theta_{i} \\
z_{i}(t) & =R_{\oplus} \cos \theta_{i},
\end{aligned}
$$

onde $R_{\oplus}$ é o raio da Terra e $\theta_{i}$ é a latitude correspondente à posição do detector. Uma vez que o nosso planeta possui sistema de rotação e translação, o ângulo $\phi_{i}(t)$ possui a seguinte dependência temporal

$$
\phi_{i}(t)=\phi_{i}(0)+\omega t-\Omega T-\pi,
$$

onde o primeiro termo do lado direito é a longitude correspondente a posição inicial do detector, o segundo termo possui a velocidade angular de rotação da Terra $(\omega)$ e o tempo $t(0 \leq t \leq 24 \mathrm{~h})$ ao instante no dia em que a SN ocorreu, dado em UTC. O terceiro termo leva em conta a velocidade de translação do planeta $(\Omega)$ em torno do Sol e o tempo $T$ é o dia do ano em que ocorre a SN, mais especificamente, é o tempo decorrido após o ponto vernal quando o detector registra os neutrinos da SN. Este tempo é comum a todos os detectores. Por simplicidade de cálculo, escolhemos como data para a chegada dos neutrinos de SN à Terra o dia 20 de março de 2000, às 12:00 UTC. A mudança para qualquer outra data e horário é simples e direta.

A posição da SN é dado pelo vetor $\boldsymbol{n}$ e a direção de propagação dos seus neutrinos é

$$
\mathbf{n}_{\mathbf{0}}=\left(n_{0 x}, n_{0 y}, n_{0 z}\right),
$$

onde

$$
\begin{aligned}
& n_{0 x}=-\cos \alpha \sin \delta \\
& n_{0 y}=-\sin \alpha \sin \delta \\
& n_{0 y}=-\cos \delta .
\end{aligned}
$$

Assim, dado dois detectores quaisquer, com vetores posição $\boldsymbol{x}_{\boldsymbol{i}}$ e $\boldsymbol{x}_{\boldsymbol{j}}$, é possível definir o vetor deslocamento como $\boldsymbol{d}_{\boldsymbol{i}} \equiv \boldsymbol{x}_{\boldsymbol{i}}-\boldsymbol{x}_{\boldsymbol{j}}$. Com estes parâmetros claros, podemos encontrar a diferença no tempo de chegada dos 
neutrinos entre estes dois detectores $\left(\Delta t_{i j} \equiv t_{i}-t_{j}\right)$ como

$$
\Delta t_{i j}=\boldsymbol{d}_{i j} \cdot \boldsymbol{n} / c
$$

onde $c$ é a velocidade da luz no vácuo ${ }^{2}$.

Então a diferença do tempo de chegada entre os detectores 1 e 2 quaisquer pode ser reescrita como

$$
\begin{aligned}
\Delta t_{12} & =\left(R_{\oplus} / c\right)\left[\left(\cos \phi_{1}(t) \sin \theta_{1}-\cos \phi_{2}(t) \sin \theta_{2}\right) n_{0 x}\right. \\
& +\left(\sin \phi_{1}(t) \sin \theta_{1}-\sin \phi_{2}(t) \sin \theta_{2}\right) n_{0 x} \\
& \left.+\left(\cos \theta_{1}-\cos \theta_{2}\right) n_{0 z}\right]
\end{aligned}
$$

Este resultado restringe os possíveis valores para $\alpha$ e $\delta$.

Neste estudo utilizamos os seguintes locais: Kamioka(K), Polo Sul(SP), Pyhäsalmi(P) e ANDES(A). Os quatro lugares estão bem separados geograficamente, sendo três no Hemisfério Norte e um no Hemisfério Sul.

Primeiramente, trabalhamos com os detectores formando pares: Kamioka e Polo Sul, Pyhäsalmi e Polo Sul, ANDES e Polo Sul, Kamioka e Pyhäsalmi, Kamioka e ANDES e, Pyhäsalmi e ANDES. Cada par consegue restringir o possível local da ocorrência SN, mas não há podem, individualmente, dizer o local. Considerando uma explosão no centro da nossa Galáxia, i.e., $\alpha=$ $17 \mathrm{~h} 42 \mathrm{~m} 27 \mathrm{~s}$ and $\delta=-28^{\circ} 55^{\prime}$, as possíveis soluções para a eq. (5.13) são mostradas na Fig. 5.12. Deste resultado, podemos perceber que para uma combinação de dois locais, a determinação da SN é restringida à uma curva fechada no céu. Se utilizarmos três detectores em locais distintos, i.e., dois conjuntos mostrados na Fig. 5.12, a possibilidade de determinar a direção da SN diminui para apenas duas posições no céu: uma verdadeira e outra falsa. Ao considerarmos quatro diferentes detectores (três pares quaisquer da Fig. 5.12), conseguimos eliminar a opção falsa e, consequentemente, encontrar a verdadeira.

$\mathrm{Na}$ prática, devido a incerteza na medição do tempo de chegada, é possível apenas apontar a direção da SN dentro de um limite. A precisão na determinação do ângulo entre a direção da SN e o eixo que liga dois detectores

\footnotetext{
${ }^{2}$ Neste trabalho não levamos em conta o possível atraso temporal devido a massa do neutrino.
} 


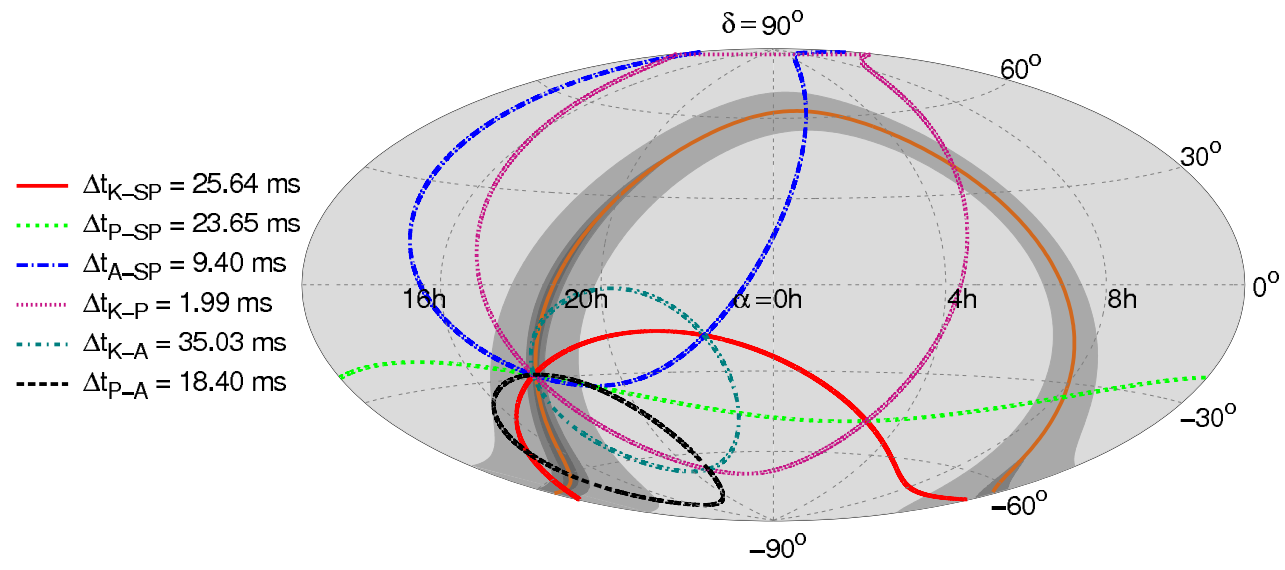

Figura 5.12: Soluções possíveis para apontar o local de ocorrência de uma SN que seja consistente com a diferença de tempo de chegada dado pela combinação de detectores organizados par a par. Coordenada correta para este exemplo é $\alpha=$ $17^{h} 42^{m} 27^{s}$ e $\delta=-28^{\circ} 55^{\prime}$.

quaisquer, $\theta$, pode ser estimada como

$$
\delta(\cos \theta) \sim \frac{c \delta\left(\Delta t_{i j}\right)}{d_{i j}}
$$

Seguindo a Ref. [160] para determinar esta precisão temporal, vamos considerar a situação onde a taxa de eventos, $N(t)$, em um dado detector, que é proporcional ao fluxo de $\nu$, aumenta (diminui) antes (depois) $t=t_{0}$ de maneira exponencial

$$
N(t)=\left\{\begin{array}{cc}
\propto \exp \left[+\frac{\left(t-t_{0}\right)}{\tau_{1}}\right] & \left(t<t_{0}\right) \\
\propto \exp \left[-\frac{\left(t-t_{0}\right)}{\tau_{2}}\right] & \left(t>t_{0}\right)
\end{array},\right.
$$

onde definimos que $\tau_{1}=30 \mathrm{~ms}$ e $\tau_{2}=3 \mathrm{~s}$, representando o tempo de subida (descida) do fluxo de neutrinos, respectivamente e, $t_{0}$ corresponde ao pico da taxa de eventos.

Seguindo esta ideia, a precisão na determinação do tempo de chegada dos neutrinos à um detector aleatório, $\delta t_{\text {arrival }}$ pode ser estimado como

$$
\delta t_{\text {arrival }} \sim \frac{\tau_{1} \tau_{2}}{\sqrt{N}} \sim \frac{\tau_{1}}{\sqrt{N_{1}}}
$$

onde $N_{1}$ é o número de eventos na parte de subida do expectro e é escrito como 


\begin{tabular}{lcccc}
\hline Detector & Massa fiducial (kt) & $N$ & $N_{1}$ & $\delta t_{\text {arrival }}(\mathrm{ms})$ \\
\hline \hline Super-K & 32 & $8.0 \times 10^{3}$ & 80 & 3.4 \\
Hyper-K & 740 & $1.9 \times 10^{5}$ & $1.9 \times 10^{3}$ & 0.7 \\
SNO+ & 0.8 & 400 & 4 & 15 \\
LENA & 44 & $1.8 \times 10^{4}$ & $1.8 \times 10^{2}$ & 2.7 \\
ANDES & 3 & $1.2 \times 10^{3}$ & 12 & 8.7 \\
IceCube & $\sim 10^{3}$ & $\sim 10^{6}$ & $\sim 10^{4}$ & 0.3 \\
\hline
\end{tabular}

Tabela 5.9: Número de eventos estimados par uma SN a $10 \mathrm{kpc}$ da Terra, assim como a precisão na determinação do tempo de chegada dos neutrinos.

$N_{1} \sim N\left(\tau_{1} / \tau_{2}\right)$ e $N$ é o número total de eventos. Observamos que quando a taxa de eventos, caracterizada pela eq. 5.15, com $\tau_{1} \ll \tau_{2}$, a fração relevante para a determinação de $\delta t_{\text {arrival }}$ é de apenas alguns $\%$.

Então, utilizando as equações fornecidas na seção 4.2 .4 calculamos o número total de eventos $N$ para a ocorrência de uma SN a uma distância típica. Para o detector de IceCube utilizamos a Ref. [137] para sabermos o número de eventos. Calculamos $N_{1}$ e a incerteza esperada no tempo de chegada, $\delta t_{\text {arrival }}$. Nossos resultados são mostrados na tabela 5.9, onde os detectores de SuperKamiokande e Hyper-Kamiokande localizam-se em Kamioka; IceCube no Polo Sul; SNO+ no Canadá; LENA em Pyhäsalmi e o laboratório de ANDES. Para os cálculos, consideramos hierarquia normal e decaimento beta inverso $\bar{\nu}_{e}+p \rightarrow$ $n+e^{+}$para todos os detectores. Adicionalmente, levamos em conta também o espalhamento elástico $\nu_{\alpha}+e^{-} \rightarrow \nu_{\alpha}+e^{-}$para os detectores Cherenkov (SuperKamiokande e Hyper-Kamiokande) e o espalhamento elástico neutrino-próton $\nu_{\alpha}+p \rightarrow \nu_{\alpha}+p$. para os cintiladores (SNO,+ LENA e ANDES).

Os resultados que encontramos para o IceCube podem ser comparados com a Ref. [161], que foi baseado em estudos de Monte Carlo. Em [161], a incerteza no tempo de chegada foi $\delta t_{\text {arrival }}=1.7 \mathrm{~ms}$ com $1 \sigma$ de nível de confiança e considerando $\tau_{1}=50 \mathrm{~ms}$. A diferença nos resultados obtidos por [161] e por nós pode ser explicado pela diferença do tempo de aumento do fluxo de neutrinos e também pelo número de eventos nos primeiros $30 \mathrm{~ms}$, que é menor em [161] $\left(\sim 6 \times 10^{3}\right)$ do que consideramos neste trabalho. Se adotarmos $\tau_{1}=50 \mathrm{~ms}$ e $N_{1} \sim 6000$, obtemos uma incerteza de $\delta t_{\text {arrival }}=0,6 \mathrm{~ms}$, que é significativamente menor que o obtido por [161]. Por isto, acreditamos que nossos resultados apresentados na tabela 5.9 podem variar por um fator de até duas vezes.

Se o tempo de queda do fluxo de neutrinos é nulo ou muito agudo, 
podemos dizer, grosseiramente, que equivale ao caso de SN Falhas, quando ocorre a formação de um buraco negro. Nesta situação, há um grande número de eventos antes do corte, em um tempo diminuto, e a incerteza no tempo de chegada é dado pelo inverso do número de eventos, antes de acontecer o corte no sinal da $\mathrm{SN}$, ou seja,

$$
\delta t_{\text {arrival }}^{\mathrm{BH}} \sim \frac{\tau}{N}
$$

onde $\tau$ é a duração do sinal e $N$ é o número total de eventos observados antes do corte no fluxo. Acredita-se [162] que a duração do sinal da SN antes da formação do buraco negro, isto é, antes do corte, seja $~ O(1)$ s. Considerando esta situação, para todos os detectores listados na tabela 5.9, com exceção do detector $\mathrm{SNO}+$, o tempo $\delta t_{\text {arrival }}$ é menor que $1 \mathrm{~ms}$. Para o $\mathrm{SNO}+$ encontramos $2.5 \mathrm{~ms}$. Entretanto, devido a incerteza na formação do buraco negro, que deve ser em torno de $0.5 \mathrm{~ms}$ [163], $\delta t_{\text {arrival }}^{B H}$ não pode ser inferior a $0.5 \mathrm{~ms}$.

Como dito acima, ao combinarmos três detectores é possível reduzir a região do céu de uma curva fechada para apenas dois pontos. O objetivo aqui é mostrar a precisão na determinação do local de ocorrência da SN. Definindo o $\chi^{2}$ como

$$
\chi^{2}=\sum_{i, j}\left[\frac{\Delta t_{i j}^{\mathrm{obs}}\left(\alpha_{0}, \delta_{0}\right)-\Delta t_{i j}^{\mathrm{theo}}(\alpha, \delta)}{\sigma_{\Delta t}}\right]^{2}
$$

onde $\Delta t_{i j}^{\text {obs }}\left(\alpha_{0}, \delta_{0}\right)$ é a diferença no tempo de chegada dos neutrinos da SN que são observados. Estes, obviamente, são as coordenadas corretas, i.e., o nosso input é $\left(\alpha_{0}, \delta_{0}\right)$ para a combinação do i-ésimo e j-ésimo detectores enquanto $\Delta t_{i j}^{\text {theo }}(\alpha, \delta)$ é a diferença de tempo de chegada teoricamente esperada para uma dada localização de SN $(\alpha, \delta)$; e $\sigma_{\Delta t}$ é a incerteza temporal estimada para $\Delta t$. Assim, logicamente, os melhores resultados ("best fit") obtidos por nosso $\chi^{2}$ é a solução da eq. (5.12) para um valor de $\Delta t_{i j}^{\text {obs }}$ como entrada.

Então, para uma SN que ocorra no centro da Via Láctea, a análise do $\chi^{2}$ considerando três detectores é mostrado na Fig. 5.13. Por simplicidade, supomos que a diferença na resolução do tempo de chegada dos neutrinos entre dois detectores quaisquer da combinação seja de $\pm 4( \pm 2)$ ms para os gráficos da coluna da esquerda (direita) na Fig. 5.13.

Conforme esperado, obtivemos sempre duas soluções possíveis para cada 

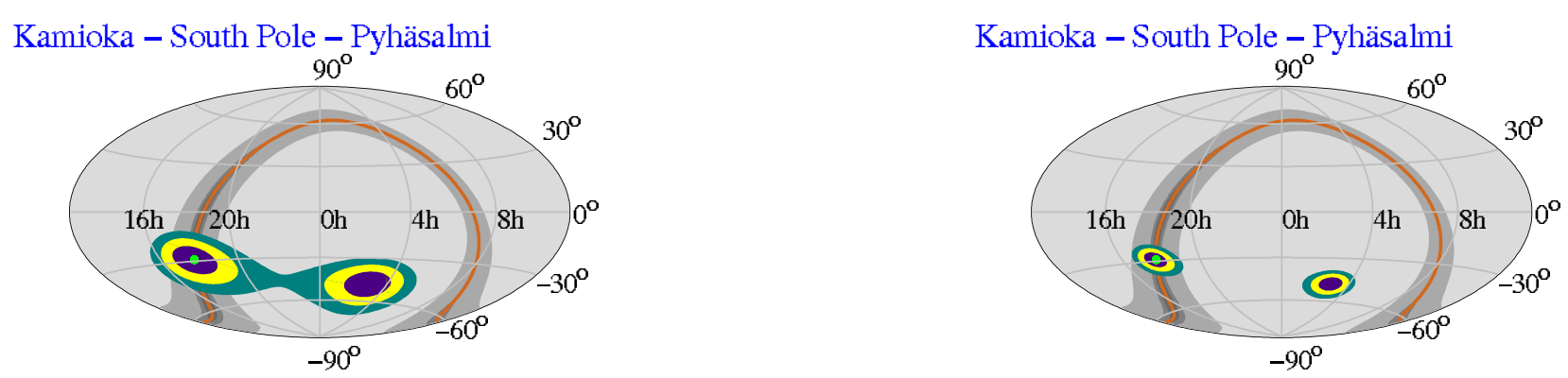

Kamioka - South Pole - Andes

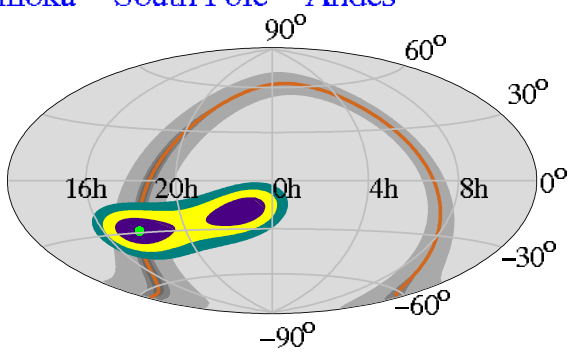

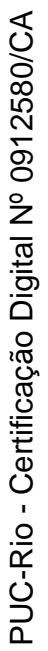

Kamioka - Pyhäsalmi - Andes

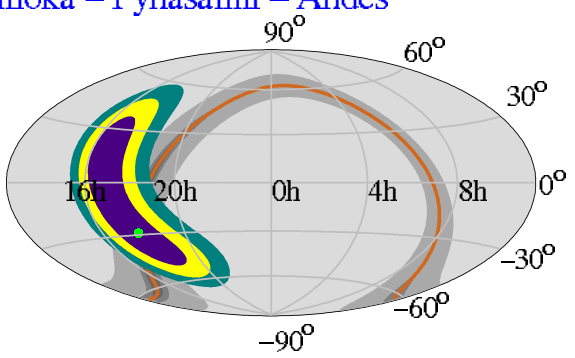

South Pole - Pyhäsalmi - Andes

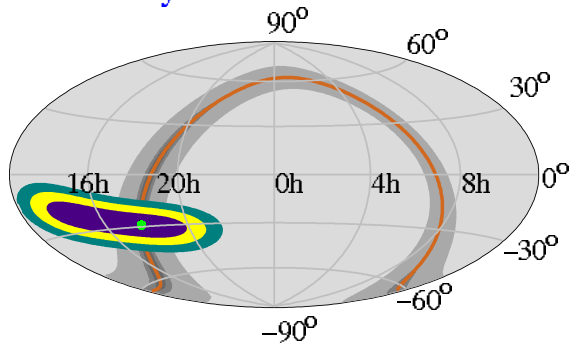

Kamioka - South Pole - Andes

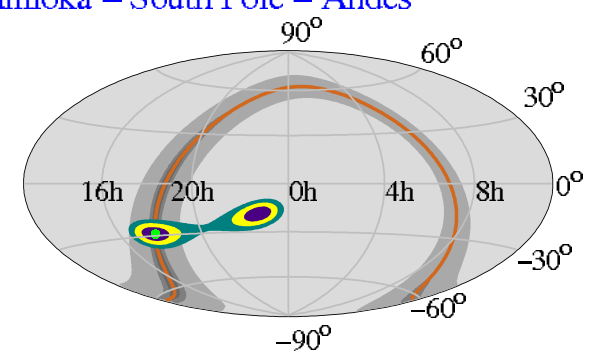

Kamioka - Pyhäsalmi - Andes

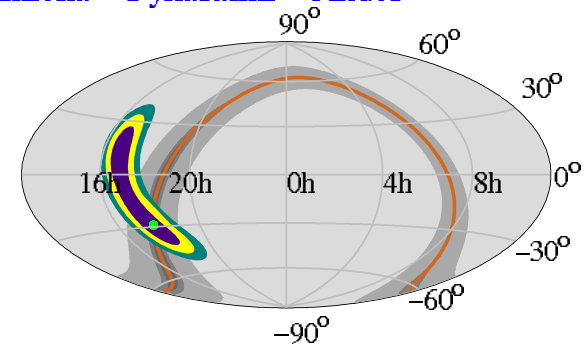

South Pole - Pyhäsalmi - Andes

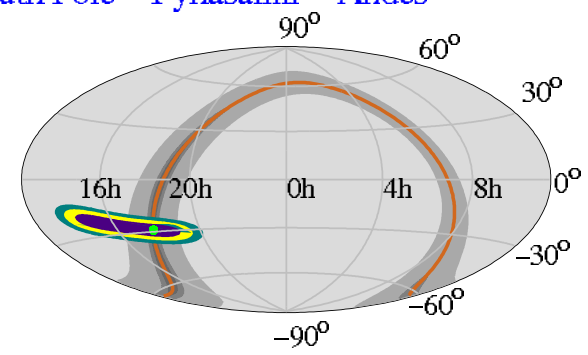

Figura 5.13: Situação onde os quatro detectores utilizados nesta tese são agrupados três a três. Cada linha é uma combinação diferente. As cores roxo, amarelo e verde indicam, respectivamente, níveis de confiança de $1 \sigma, 2 \sigma$ e $3 \sigma$. Supomos que a incerteza na medida da diferença temporal entre dois detectores é $\pm 4 \mathrm{~ms}$ para a coluna da esquerda e $\pm 2 \mathrm{~ms}$ para a da direita.

combinação de três detectores, ou seja, uma posição no céu é a verdadeira e outra é falsa. Observamos que as opções verdadeira e falsa são conectadas dentro de $1 \sigma$ de nível de confiança para as combinações Kamioka - Pyhäsalmi 

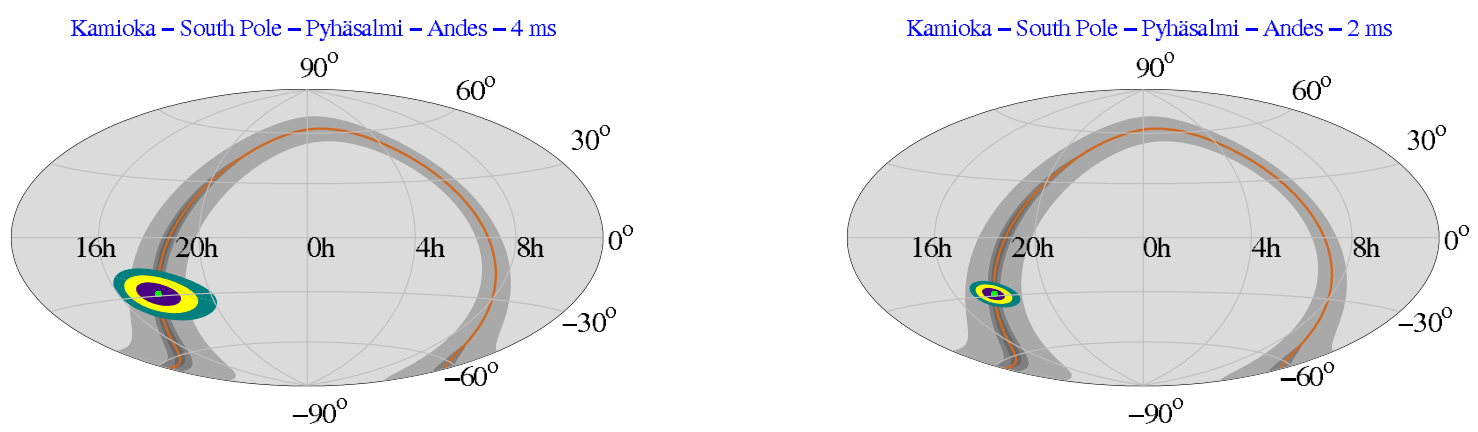

Figura 5.14: Situação onde os quatro detectores utilizados nesta tese são agrupados três a três. Cada linha é uma combinação diferente. As cores roxo, amarelo e verde indicam, respectivamente, níveis de confiança de $1 \sigma, 2 \sigma$ e $3 \sigma$. Supomos que a incerteza na medida da diferença temporal entre dois detectores é $\pm 4 \mathrm{~ms}$ para a coluna da esquerda e \pm 2 ms para a da direita.

- ANDES e Polo Sul - Pyhäsalmi - ANDES, respectivamente, terceira e quarta linhas da Fig. 5.13. Embora não saibamos qual é a verdadeira posição da SN, sabemos que a mais provável é a que recai no Disco Galáctico.

Ao fazermos a combinação com quatro detectores situados em posições geográficas distintas, eliminamos a opção falsa. A Fig. 5.14 mostra o nosso resultado considerando a ocorrência de uma SN no centro do Disco Galáctico e a resolução temporal de $\pm 4 \mathrm{~ms}$ (esquerda) e $\pm 2 \mathrm{~ms}$ (direita).

Na Fig. 5.15 é possível observar o resultado do $\chi^{2}$ considerando o lado oposto do centro da Galáxia como posição da explosão da SN, i.e., $\alpha=$ $5^{\mathrm{h}} 42^{\mathrm{m}} 27^{\mathrm{s}}$ e $\delta=28^{\circ} 55^{\prime}$. De ambas as figuras podemos inferir uma precisão de $\Delta(\alpha) \sim 15(8)^{\circ}$ e $\Delta(\delta) \simeq 10(5)^{\circ}$ para a resolução temporal de $\pm 4 \mathrm{~ms}$ (2 $\mathrm{ms})$.

Em suma, vemos que utilizando somente dois detectores, há uma vasta região no céu onde pode ter ocorrido a SN, tornando muito improvável o seu encontro antes da chegada dos fótons. Isto pode ser visto na Fig. 5.12, embora nesta figura não levamos em conta a incerteza no tempo de chegada, $\delta t_{\text {arrival }}=0$. Esta área é significativamente reduzida ao adicionarmos um terceiro detector (Fig. 5.13). E o introduzirmos um quarto detector (Figs. 5.14 e 5.15), a região permitida sofre uma redução adicional de $\sim 50 \%$.

Embora estes resultados de resolução angular ainda sejam muito aquém daqueles obtidos com o uso do telescópio ótico, lembramos que muitas das SN ocorridas dentro da Via Láctea não são detectadas oticamente devido a poeira interestelar, por exemplo, que enfraquecem muito o sinal dos fótons que 

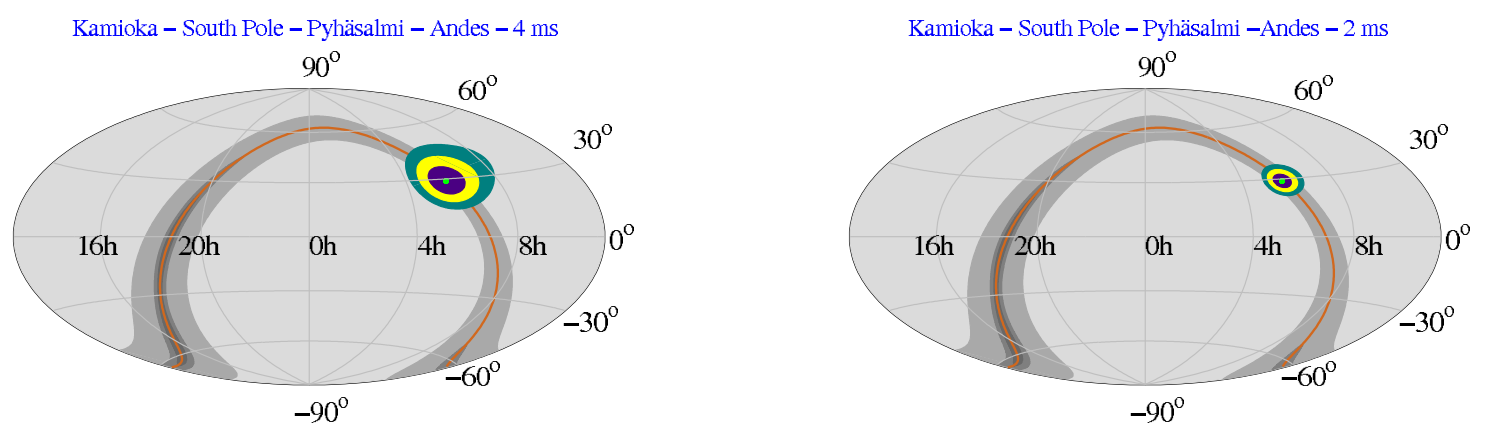

Figura 5.15: Idem a Fig. 5.15, mas para a explosão de SN localizada na posição oposta ao centro da Via Láctea.

chegam à Terra, ou ainda no caso de SN Falhas, quando possivelmente não há sinal ótico. Assim, chamamos a atenção para a possibilidade da obtenção da posição de uma SN galáctica utilizando apenas os dados registrados pelos seus neutrinos e permitindo que tal informação seja repassada à comunidade astronômica em tempo de ser observada o surgimento do espectro da SN. 


\section{6}

\section{Conclusão}

Este tese teve por objetivo mostrar a importância científica da construção de um detector de neutrinos na Cordilheira dos Andes para o estudo e detecção de neutrinos oriundos de uma SN galáctica, que propiciará uma melhor compreensão dos mecanismos de explosão de uma SN e/ou elucidará algumas das propriedades dos neutrinos que ainda são desconhecidas da comunidade científica hodierna.

O laboratório subterrâneo de ANDES, se construído, será o primeiro desta categoria no Hemisfério Sul e, em especial, da América do Sul. A Cordilheira dos Andes fornece uma blindagem natural de 4,5 km de águaequivalente, uma das melhores possíveis, que é excelente para a execução de vários experimentos que necessitam de proteção contra raios cósmicos ou de neutrons provenientes de espalhamentos de raios cósmicos. Com ele, será possível investigar eventos e decaimentos raros, onde é necessário um ambiente com baixíssimo ruído natural, assim como interações fracas (matéria escura ou com neutrinos). Neste quesito, o estudo de neutrinos solares é interessante já que há indícios da possibilidade de detecção com cintiladores; e propostas na procura de sinais indiretos de matéria escura através da detecção de neutrinos que provém da aniquilação da matéria escura.

Além da blindagem oferecida pela Cordilheira, o laboratório de ANDES tem em sua localização geográfica uma característica muito importante: a quase ausência de reatores nucleares na proximidade, proporcionando um baixo ruído devido a neutrinos de reatores. Esta é uma excelente condição para o estudo/detecção de geoneutrinos, i.e., neutrinos produzidos no interior na Terra devido ao decaimento beta de elementos radioativos como Tório e Urânio. Outras áreas da ciência também poderão ser alocadas no laboratório de ANDES como, por exemplo, astrofísica nuclear, estudo de vida/biosistemas sob condições extremas, geologia, geofísica, etc. 
Em específico, neste trabalho estudamos o potencial de um detector de neutrinos utilizando líquido cintilador com uma massa fiducial de $3 \mathrm{kt}$ para a observação de neutrinos oriundos da próxima SN galáctica. Devido a baixa ocorrência de SN locais, é importante que tenhamos o máximo possível de detectores em funcionamento para que sejam coletados o maior número possível de eventos. Considerando a distância de $10 \mathrm{kpc}$, obtivemos um total de eventos de $\sim 750$ para o detector de ANDES para o decaimento beta inverso e sem oscilação. Levando em conta o fenômeno oscilatório, a previsão para a mesma reação é de registro de 800 - 1000 eventos, dependendo da hierarquia de massa e energia média dos neutrinos não-eletrônicos. O detector de ANDES também pode observar uma faixa entre $\sim 450-650$ eventos para o espalhamento elástico próton neutrino com energia cinética de recúo do próton acima de $0.2 \mathrm{MeV}$. Como esta é uma reação de corrente neutra, a variação no número de eventos deve-se a diferentes estimativas para $\left\langle E_{\nu_{x}}\right\rangle$.

Estudamos a possibilidade de determinação do fluxo original de neutrinos através da reconstrução do fluxo utilizando os dados obtidos através do espalhamento elástico entre o neutrino e o próton. Nosso resultado foi de precisão de $15 \%$ para neutrinos com energia $\sim 20-40 \mathrm{MeV}$.

Depois, verificamos com que precisão podemos determinar $\left\langle E_{\nu_{x}}\right\rangle$. Obtivemos que quanto mais distante $\left\langle E_{\nu_{x}}\right\rangle$ for $\left\langle E_{\bar{\nu}_{e}}\right\rangle$, melhor será a precisão na medida. Por exemplo, para $\left\langle E_{\nu_{x}}\right\rangle=18 \mathrm{MeV}$ e $E_{\nu_{x}}^{\text {Tot }}=5 \times 10^{52} \mathrm{erg}$, a precisão foi de $6 \%(13 \%)$ e $17 \%$ (40\%), respectivamente, para 1 (3) $\sigma$.

Na estimativa da razão entre corrente carregada e corrente neutra, i.e., $R\left(N_{\bar{\nu}_{e} p} / N_{\nu p}\right)$, em função da $\left\langle E_{\nu_{x}}\right\rangle$ ou $\left\langle E_{\bar{\nu}_{e}}\right\rangle$ verificamos que é possível distinguir entre hierarquia normal e invertida se $\left\langle E_{\nu_{x}}\right\rangle$ e $\left\langle E_{\bar{\nu}_{e}}\right\rangle$ tiverem uma diferença significativa. Para $R\left(N_{\bar{\nu}_{e} p} / N_{\nu p}\right)$ em função da probabilidade de sobrevivência, $\bar{p}$, é mais fácil distinguir $\bar{p}$ se a hierarquia for invertida. E comparando com a razão entre as luminosidades, é possível identificar a hierarquia de massa caso $L_{\bar{\nu}_{e}} / L_{\nu_{x}}$ for abaixo de $\sim 0.7$ ou acima de $\sim 1.5$.

Em particular, o detector de ANDES aumenta significativamente as chances de observação do efeito de matéria da Terra ao combinar seus dados com os dos detectores localizados no Hemisfério Norte. Obtivemos que a probabilidade de sombreamento da Terra, passando pelo manto, em ao menos um detector do conjunto quando combinamos detectores é de $\sim 72 \%, \sim 96 \%$ e ultrapassa $98 \%$ quando temos 2, 3 e 4 detectores em locais distintos. Para o manto, o conjunto de 4 detectores, incluindo o detector de ANDES, chega a 
consideráveis $34 \%$.

O detector de ANDES também pode vir a ser integrante do sistema SNEWS, a rede internacional de alerta para a ocorrência de SN, já que há a possibilidade da utilização do detector, em conjunto com outros detectores ao redor do globo, na localização de uma SN galáctica apenas através da observação de seus neutrinos. A determinação da posição da SN pode ser alcançada através da técnica de triangulação ao compararmos o tempo de chegada dos neutrinos nos diferentes detectores. A combinação dos tempos de chegada de quatro detectores é suficiente para a determinação do local da SN e a resolução temporal é comandada pelo menor detector, já que esta incerteza está diretamente ligada a quantidade de eventos observados. Dentro de uma incerteza temporal de $\sim 2$ (4) ms, utilizando os detectores localizados em Kamioka (SuperKamiokande) e Polo Sul (IceCube) - já existentes - e de Pyhäsalmi (LENA, GLACIER ou MEMPHIS) e ANDES - ambos propostos para um futuro próximo - estabelecemos uma precisão angular de $\sim 5(10)^{\circ}$ e $8(15)^{\circ}$ na declinação e ascenção reta, respectivamente. Isto pode ser particularmente importante no caso de "Supernovas Falhas", que são as SN de grande massa e formam buracos negros ao colapsarem antes da ocorrência da explosão propriamente dita, uma vez que neste tipo de SN não há a emissão de fótons, logo não há como ter observação astronômica. 


\section{Referências Bibliográficas}

[1] J. Chadwick. Verh. d. deutschen Phys. Ges., 16:383, 1914. 1

[2] O. von Baeyer and O. Hahn. Phys. Zs., 11:488, 1910. 1

[3] O. Hahn and L. Meitner. Phys. Zs., 9:321, 1908. 1

[4] O. Hahn and L. Meitner. Phys. Zs., 9:697, 1908. 1

[5] C. D. Ellis and W. A. Wooster. Proc. Roy. Soc. A, 117:109, 1927. 1

[6] N. Bohr, H. A. Kramers and J. C. Slater. Über die Quantentheoire der Strahlung. Z. Physik, 24:69-87, 1924. 1

[7] W. Pauli. Offener Brief an die Gruppe der Radioaktiven bei der Gauvereins -Tagung zu Tübingen. 1930. 1

[8] Chadwick, J. Possible Existence of a Neutron. Nature, 129:312, 1932. 1

[9] Laboratoire d'Annecy-le-Vieux de Physique des Particules. http://lappweb.in2p3.fr/neutrinos/anhistory.html. 1

[10] Reines, F. and Cowan, C. L. Detection of the free neutrino. Phys. Rev., 92:830-831, 1953. 1

[11] Cowan, C. L., Reines, F., Harrison, F. B., Kruse, H. W., and McGuire, A. D. Detection of the free neutrino: A Confirmation. Science, 124:103-104, 1956. 1

[12] Konopinski, E. J. and Mahmoud, H. M. The Universal Fermi interaction. Phys. Rev., 92:1045-1049, 1953. 1

[13] Danby, G. and others. Observation of High-Energy Neutrino Reactions and the Existence of Two Kinds of Neutrinos. Phys. Rev. Lett., 9:36-44, 1962. 1 
[14] Perl, Martin L. and others. Evidence for anomalous lepton production in e+ e- annihilation. Phys. Rev. Lett., 35:1489-1492, 1975. 1

[15] K. Kodama and et. al. for DONUT Collaboration. Observation of tauneutrino interactions. Phys. Lett., B504:218-224, 2001. 1

[16] Cleveland, B. T. and others. Measurement of the solar electron neutrino flux with the Homestake chlorine detector. Astrophys. J., 496:505-526, 1998. 1

[17] Hirata, K. S. and others. Observation of a small atmospheric $\nu_{\mu} / \nu_{e}$ ratio in Kamiokande. Phys. Lett., B280:146-152, 1992. 1

[18] Becker-Szendy, R. and others. A Search for muon-neutrino oscillations with the IMB detector. Phys. Rev. Lett., 69:1010-1013, 1992. 1

[19] Becker-Szendy, R. and others. Neutrino measurements with the IMB detector. Nucl. Phys. Proc. Suppl., 38:331-336, 1995. 1

[20] K. Eguchi et. al. for KamLAND Collaboration. First results from KamLAND: Evidence for reactor antineutrino disappearance. Phys. Rev. Lett., 90:021802, 2003. 1, 4.2.2

[21] Fukuda, Y. and others. Evidence for oscillation of atmospheric neutrinos. Phys. Rev. Lett., 81:1562-1567, 1998. 1, 4.1.2, 5.1

[22] P. A. N. Machado, T. Mühlbeier, H. Nunokawa, and R. Zukanovich Funchal. Potential of a neutrino detector in the andes underground laboratory for geophysics and astrophysics of neutrinos. Phys. Rev. D, 86:125001, 2012. 1, 4.3, 5.4

[23] X. Bertou. Third ANDES workshop. In Third ANDES Workshop, Valparaiso - Chile, 2012. 1, 4.1.4

[24] X. Bertou. The ANDES underground laboratory. The European Physical Journal Plus, 127:1-6, 2012. 1, 4.1.4

[25] Laurence A. Marschall. The Supernova Story. Plenum, 233 Spring Street, New York, N.Y. 10013, 1988. 2.1 
[26] Jacco Vink, Johan Bleeker, Kurt van der Heyden, Andrei Bykov, Aya Bamba, and Ryo Yamazaki. The x-ray synchrotron emission of RCW 86 and the implications for its age. The Astrophysical Journal Letters, 648:L33, 2006. 2.1

[27] Wheeler, J. Craig. Observations and theory of supernovae. astroph/0209514v1, 2002. 2.1

[28] Acero, F. et al. and HESS collaboration. First detection of VHE s from SN1006 by HESS. Astronomy and Astrophysics, 516:A62, 2010. 2.1

[29] Una Hwang, Anne Decourchelle, Stephen S. Holt, and Robert Petre. Thermal and nonthermal emission from the forward shock in tycho's supernova remnant. Astrophys.J., 581:1101-1115, 2002. 2.1

[30] Suzuki, Hideyuki. Physics and Astrophysics of Neutrinos. Springer Verlag, 1994. 2.1, 2.3, 2.3, 2.4.2, 4.2.2

[31] Bethe, Hans Albrecht . Supernova mechanisms. Review of Modern Physics, 62:66, 1990. 2.1, 2.4.2, 2.4.3, 2.4.3, 2.4.3

[32] Baade, W. and Qwicky, F. Remarks on super-novae and cosmic rays. Phys. Rev., 46:2, 1934. 2.1

[33] H. Guerlac and M. Jacob. Bentley, newton and providence (the boyle lectures once more). Journal of the History of Ideas, 30:307-319, 1969. 2.2 .1

[34] N. Murray. Star formation efficiencies and lifetimes of giant molecular clouds in the milky way. The Astrophysical Journal, 729:133, 2011. 3

[35] Zeilik, M, Gregory, S. A., and Smith, P. Introductory Astronomy and Astrophysics. Saunders College Publishing, 1992. 2.2.1, 2.2.3

[36] Stefano Lecchini. How Dwarfs Become Giants: The Discovery of the Mass-Luminosity Relation. Bern Studies, 2007. 2.2.2

[37] Maurizio Salaris and Santi Cassisi. Evolution of Stars and Stellar Populations. Wiley, 2005. 2.2.2

[38] Giunti, C. and Kim, Chung W. Fundamentals of Neutrino Physics and Astrophysics. Oxford University Press, 2007. 2.3, 2.3, 2.4.5, 2.7, 3.4.1 
[39] Shapiro, Stuart L. and Teulolsky, Saul A. Black Holes, White Dwarfs and Neutron Stars. Wiley-Interscience Publication, 1983. 2.3, 2.3

[40] Chandrasekhar, S. An Introduction to the Study of Stellar Structure. University of Chicago Press, 1938. 2.3

[41] Nomoto, K. et. al. Frontiers of neutrino astrophysics. In Proceedings of the International Symposium on Neutrino Astrophysics, page 235, 1993. 2.3

[42] Wheeler, J. C., Harkness, R. P., and Cappellaro, E. World Scientific, Singapure, 1987. 2.3

[43] Berg, S. van den and Tammann, G. A. Galactic and extragalactic supernova rates. Ann. Rev. Astr. Astrophys., 29:363-407, 1991. 2.3, 5.1

[44] Montes, M. J. Supernova taxonomy. http://rsdwww.nrl.navy.mil/7212/montes/snetax.html. 10

[45] Cappellaro, E. and Turatto, M. The influence of binaries on stellar population studies. astro-ph/0012455, 2000. 10

[46] Heger, A., Fryer, C. L., Woosley, S. E., Langer, N., and Hartmann, D. H. How massive single stars end their life. Astrophys. J., 591:288, 2003. $2.3,2.4 .2$

[47] Woosley, S. E. and Weaver, T. A. The Physics of Supernova Explosions. Ann. Rev. Astron. Astrophys., 24:205-253, 1986. 2.4.1, 2.4.2

[48] A. J. T. Poelarends, F. Herwig, N. Langer, and A. Heger. The supernova channel of super-agb stars. The Astrophysical Journal, 675:614, 2008. 2.4 .2

[49] S. Wanajo, K. Nomoto, H.-T. Janka, F. S. Kitaura, and B. Müller. Nucleosynthesis in electron capture supernovae of asymptotic giant branch stars. The Astrophysical Journal, 695:208, 2009. 2.4.2

[50] Shinya Wanajo, Hans-Thomas Janka, and Bernhard Müller. Electroncapture supernovae as the origin of elements beyond iron. The Astrophysical Journal Letters, 726:L15, 2011. 2.4.2 
[51] S.E. Woosley, A. Heger, and T.A. Weaver. The evolution and explosion of massive stars. Rev.Mod.Phys., 74:1015-1071, 2002. 2.4.2

[52] Freedman, D. Z. Phys. Rev. D, 9:1389, 1974. 2.4.3

[53] Sato, K. Neutrino degeneracy in supernova cores and neutral current of weak interaction. Prog. Theor. Phys., 53:595-597, 1975. 2.4.3

[54] Sato, K. Supernova Explosion and Neutral Currents of Weak Interaction. Prog. Theor. Phys., 54:1325-1338, 1975. 2.4.3

[55] Mazurek, T. J. Chemical potential effects on neutrino diffusion in supernovae. Astrophys. Sp. Sci., 35:117-135, 1975. 2.4.3

[56] Burrows, Adam, Reddy, Sanjay, and Thompson, Todd A. Neutrino opacities in nuclear matter. Nuclear Physics A, 777:356 - 394, 2006. Special Isseu on Nuclear Astrophysics. 2.4.3

[57] Beringer, J. et al. Particle Data Group. Phys. Rev. D, 86:010001, 2012. $11,3.2 .1,3.8 .1$

[58] Kotake, K., Sato, K., and Takahashi, K. Explosion mechanism, neutrino burst and gravitational wave in core-collapse supernovae. Rep. Prog. Phys., 69:971-1143, 2006. 2.4.3, 2.4.5

[59] W. D. Arnett. Astrophys. J., 218:215, 1977. 2.4.4

[60] Goldreich, P. and Weber, S. V. Homologously Collapsing Stellar Cores. Astrophys. J., 238:991-997, 1980. 2.4.4

[61] Yahil, A. and Lattimer, J. M. D. Reidel Publishing Company, Dordrecht, 1982. 2.4.4

[62] Baron, E. A., Cooperstein, J., and Kahana, S. Phys. Rev. Lett., 55:126, 1985. 2.4 .4

[63] Baron, E. A., Cooperstein, J., and Kahana, S. Nucl. Phys. A, 440:744, 1985. 2.4 .4

[64] S. W. Bruenn. Astrophys. J. Suppl., 58:771-841, 1985. 2.4.4

[65] Arnett, W. D. Neutrino escape, nuclear dissociation, and core collapse and/or explosion. Astrophys. J., 263:55-57, 1983. 2.4.5 
[66] Hillbrandt, W. et al. Supernova explosions of massive stars - The mass range 8 to 10 solar masses. Astron. Astrophys., 133:175, 1984. 2.4.5

[67] Burrows, A. and Thompson, T. A. The mechanism of core-collapse supernova explosions: A status report. astro-ph/0212469v1, 2002. 2.4.5

[68] Wilson, J. R. Numerical Astrophysics. Jones \& Barlettt, Boston, 1985. 2.4 .5

[69] Rampp, Markus and Janka, H. Thomas. Spherically symmetric simulation with Boltzmann neutrino transport of core collapse and post-bounce evolution of a 15 solar mass star. Astrophys. J., 539:L33-L36, 2000. 2.4.5

[70] Janka, H. T. Core-collapse supernovae: Successes, problems, and perspectives. Nucl. Phys., A663:119-131, 2000. 2.4.5

[71] Liebendoerfer, Matthias and others. Probing the gravitational well: No supernova explosion in spherical symmetry with general relativistic Boltzmann neutrino transport. Phys. Rev., D63:103004, 2001. 2.4.5

[72] Burrows, Adam, Livne, Eli, Dessart, Luc, Ott, Christian, and Murphy, Jeremiah. A New Mechanism for Core-Collapse Supernova Explosions. Astrophys. J., 640:878-890, 2006. 2.4.5

[73] Bruenn, S. W. and others. Modeling core collapse supernovae in 2 and 3 dimensions with spectral neutrino transport. J. Phys. Conf. Ser., 46:393402, 2006. 2.4.5

[74] Janka, H. and Hillebrandt, W. Neutrino emission from type II supernovae - an analysis of the spectra. Astron. Astroph., 224:49-56, 1989. 2.5

[75] Dighe, Amol S., Keil, Mathias T., and Raffelt, Georg G. Detecting the neutrino mass hierarchy with a supernova at IceCube. JCAP, 0306:005, 2003. $2.5,5.4$

[76] Keil, Mathias Th., Raffelt, Georg G., and Janka, Hans-Thomas. Monte Carlo study of supernova neutrino spectra formation. Astrophys. J., 590:971-991, 2003. 2.5

[77] M.T. Keil, G.G. Raffelt and H.T. Janka. Astrophys. J., 590:971, 2003. 2.5 
[78] Mathias Th. Keil. Supernova Neutrino Spectra and Applications to Flavor Oscillations. PhD thesis, Max-Planck-Institut f. Physik, Muenchen, Germany, 2003. 2.5, 5.1

[79] R. Buras, H.T. Janka, M.T. Keil, G.G. Raffelt, and M. Rampp. Astrophys. J., 587:320, 2003. 2.5

[80] A. Mirizzi, G.G. Raffelt, and P.D. Serpico. Earth matter effects in supernova neutrinos: optimal detector locations. Journal of Cosmology and Astroparticle Physics, 2006:012, 2006. 2.6, 2.6, 5.4, 5.4

[81] I. Yusifov and I. Küçük. Revisiting the radial distribution of pulsars in the galaxy. Astron. Astroph., 422:545-553, 2004. 2.6

[82] T. Mühlbeier, H. Nunokawa, and R. Zukanovich Funchal. Revisiting the triangulation method for pointing to supernova and failed supernova with neutrinos. Phys. Rev. D, 88:085010, 2013. 2.6

[83] International Astronomical Union. Circular No. 4317. 1987. 2.7

[84] Middleditch, J., Kristian, J. A., Kunkel, W. E., Hill, K. M., Watson, R. M., Luicio, R., Imamura, J. N., Steiman-Cameron, T. Y., Shearer, A., Redfern, M., Butler, R., and Danks, A. C. A 2.14 ms candidate optical pulsar in SN1987A. astro-ph/0010044. 2.7

[85] Hirata, K. S., Kajita, T., Koshiba, M., Nakahata, M., Oyama, Y., Sato, N., Suzuki, A., Takita, M., Totsuka, Y., Kifune, T., Suda, T., Takahashi, K., Tanimori, T., Miyano, K., Yamada, M., Beier, E. W., Feldscher, L. R., Frati, W., Kim, S. B., Mann, A. K., Newcomer, F. M., Van Berg, R., Zhang, W., and Cortez, B. G. Observation in the kamiokande-ii detector of the neutrino burst from supernova sn1987a. Phys. Rev. D, 38:448-458, 1988. 2.7

[86] Hirata, K., Kajita, T., Koshiba, M., Nakahata, M., Oyama, Y., Sato, N., Suzuki, A., Takita, M., Totsuka, Y., Kifune, T., Suda, T., Takahashi, K., Tanimori, T., Miyano, K., Yamada, M., Beier, E. W., Feldscher, L. R., Kim, S. B., Mann, A. K., Newcomer, F. M., Van, R., Zhang, W., and Cortez, B. G. Observation of a neutrino burst from the supernova sn1987a. Phys. Rev. Lett., 58:1490-1493, 1987. 2.7 
[87] Bionta, R. M., Blewitt, G., Bratton, C. B., Casper, D., Ciocio, A., Claus, R., Cortez, B., Crouch, M., Dye, S. T., Errede, S., Foster, G. W., Gajewski, W., Ganezer, K. S., Goldhaber, M., Haines, T. J., Jones, T. W., Kielczewska, D., Kropp, W. R., Learned, J. G., LoSecco, J. M., Matthews, J., Miller, R., Mudan, M. S., Park, H. S., Price, L. R., Reines, F., and Schultz, J. . Observation of a neutrino burst in coincidence with supernova 1987a in the large magellanic cloud. Phys. Rev. Lett., 58:14941496, 1987. 2.7

[88] Bratton, C. B., Casper, D., Ciocio, A., Claus, R., Crouch, M., Dye, S. T., Errede, S., Gajewski, W., Goldhaber, M., Haines, T. J., Jones, T. W., Kielczewska, D., Kropp, W. R., Learned, J. G., LoSecco, J. M., Matthews, J., Miller, R., Mudan, M., Price, L. R., Reines, F., Schultz, J., Seidel, S., Sinclair, D., Sobel, H. W., Stone, J. L., Sulak, L., and Svoboda, R. Angular distribution of events from sn1987a. Phys. Rev. D, 37:3361-3363, 1988. 2.7

[89] Alexeyev, E. N. and Alexeyeva, L. N. and Krivosheina, I. V. and Volchenko, V. I. Detection of the neutrino signal from SN1987A in the LMC using the INR Baksan underground scintillation telescope. Physics Letters B, 205:209 - 214, 1988. 2.7

[90] Dadykin, V. L. and et al. JETP Lett., 45:593-595, 1987. 2.7

[91] Loredo, Thomas J. and Lamb, Donald Q. Bayesian analysis of neutrinos observed from supernova SN1987A. Phys. Rev. D, 65:063002, 2002. 2.7

[92] Pontecorvo, B. Sov. Phys. JETP, 6:429, 1957. 3

[93] Pontecorvo, B. Sov. Phys. JETP, 7:172-173, 1958. 3

[94] Maki, Z., Nakagawa, M., and Sakata, S. Remarks on the unified model of elementary particles. Prog. Theor. Phys., 28:870, 1962. 3

[95] Eliezer, S. and Swift, A. R. Nucl. Phys. B, 105:45, 1976. 3

[96] Fritzsch, H. and Minkowski, P. Phys. Lett. B, 62:72, 1976. 3

[97] Bilenky, S. M. and Pontecorvo, B. Sov. J. Nucl. Phys., 24:316-319, 1976. 
[98] Kobayashi, Makoto and Maskawa, Toshihide. CP Violation in the Renormalizable Theory of Weak Interaction. Prog. Theor. Phys., 49:652657, 1973. 3.1

[99] Halzen, F. and Martin, B. R. Quarks and Leptons: an Introductory Course in Modern Particle Physics. John Wiley \& Sons, 1984. 3.1

[100] Martin, B. R. and Shaw, G. Particle Physics. John Wiley \& Sons, 1992. 3.1

[101] Griffiths, D. J. Introduction to Elementary Particles. John Wiley \& Sons, 1987. 3.1

[102] King, S. F. Neutrino mass models. REPT.PROG.PHYS., 67:107, 2004. 3.2 .1

[103] Samoil M. Bilenky, J. Hosek, and S.T. Petcov. On Oscillations of Neutrinos with Dirac and Majorana Masses. Phys.Lett., B94:495, 1980. 3.2 .1

[104] M. Doi, T. Kotani, H. Nishiura, K. Okuda, and E. Takasugi. Majorana Neutrinos and mu Decay. Prog.Theor.Phys., 67:281, 1982. 3.2.1

[105] P. Langacker, S.T. Petcov, G. Steigman, and S. Toshev. On the MikheevSmirnov-Wolfenstein (MSW) Mechanism of Amplification of Neutrino Oscillations in Matter. Nucl.Phys., B282:589, 1987. 3.2.1

[106] Wolfenstein, L. Neutrino oscillations in matter. Phys. Rev. D, 17:23692374, 1978. 3.4, 3.4.1, 3.6

[107] Langacker, P., Petcov, S. T., Steigman, G., and Toshev, S. Nucl. Phys.B, 282:589, 1987. 3.5

[108] Bilenky, S. M., Hosek, J., and Petcov, S. T. Phys. Lett. B, 94:495, 1980. 3.5

[109] Doi, M., Kotani, T., Nishiura, H., Okuda, K., and Takasugi, E. Phys. Lett. B, 102:323, 1981. 3.5

[110] Mikheev, S. P. and Smirnov, A. Yu. Resonance enhancement of oscillations in matter and solar neutrino spectroscopy. Sov. J. Nucl. Phys., 42:913-917, 1985. 3.6 
[111] Dighe, Amol S. and Smirnov, Alexei Yu. Identifying the neutrino mass spectrum from the neutrino burst from a supernova. Phys. Rev.D, 62:033007, 2000. 3.7.1, 3.8, 3.8.1, 3.8.1, 5.1, 5.4

[112] Landau, L. Phys. Z. Sowjetunion, 2:235, 1932. 3.7.2

[113] Zener, C. Proc R. Soc. London, volume 137 of Ser. A. 1932. 3.7.2

[114] F. P. An et. al. for Daya Bay Collaboration. Observation of electronantineutrino disappearance at daya bay. Phys. Rev. Lett., 108:171803, 2012. 3.8.1, 3.9

[115] J. K. Ahn et. al. for the RENO Collaboration. Observation of reactor electron antineutrinos disappearance in the reno experiment. Phys. Rev. Lett., 108:191802, 2012. 3.8.1, 3.9

[116] Y. Abe et. al. Indication of reactor $\bar{\nu}_{e}$ disappearance in the double chooz experiment. Phys. Rev. Lett., 108:131801, 2012. 3.9

[117] C.V. Achar et. al. Detection of muons produced by cosmic ray neutrinos deep underground. Physics Letters, 18:196 - 199, 1965. 4.1

[118] F. Reines, M.F. Crouch, T.L. Jenkins, W.R. Kropp, H.S. Gurr, et al. Evidence for high-energy cosmic ray neutrino interactions. Phys.Rev.Lett., 15:429-433, 1965. 4.1

[119] R. Davis. Solar neutrinos. II: Experimental. Phys.Rev.Lett., 12:303-305, 1964. 4.1

[120] Joseph A. Formaggio and C. J. Martoff. Backgrounds to sensitive experiments underground. Annual Review of Nuclear and Particle Science, 54:361-412, 2004. 4.1

[121] Q. R. Ahmad et. al. Direct evidence for neutrino flavor transformation from neutral-current interactions in the sudbury neutrino observatory. Phys. Rev. Lett., 89:011301, 2002. 4.1.3, 4.2.3, 5.1

[122] G. Alimonti et. al. for the Borexino Collaboration. Science and technology of Borexino: a real-time detector for low energy solar neutrinos. Astroparticle Physics, 16:205 - 234, 2002. 4.2.1, 4.2.1 
[123] L Cadonati and F.P Calaprice and M.C Chen. Supernova neutrino detection in Borexino. Astroparticle Physics, 16:361 - 372, 2002. 4.2.1

[124] K. S. Hirata et. al. Observation in the Kamiokande-II detector of the neutrino burst from supernova SN1987A. Phys. Rev. D, 38:448-458, 1988. 4.2 .2

[125] J. Boger et. al. for the SNO Collaboration. The Sudbury Neutrino Observatory. Nuclear Instruments and Methods in Physics Research Section A: Accelerators, Spectrometers, Detectors and Associated Equipment, $449: 172-207,2000.4 .2 .3$

[126] Q. R. Ahmad et. al. for SNO Collaboration. Measurement of Day and Night Neutrino Energy Spectra at SNO and Constraints on Neutrino Mixing Parameters. Phys. Rev. Lett., 89:011302, 2002. 4.2.3

[127] C. Kraus. SNO with liquid scintillator: $\mathrm{SNO}+$. Progress in Particle and Nuclear Physics, 57:150 - 152, 2006. 4.2.3, 5.2

[128] Christine Kraus and Simon J.M. Peeters. The rich neutrino programme of the SNO+ experiment. Progress in Particle and Nuclear Physics, $64: 273$ - $277,2010.4 .2 .3$

[129] Alessandro Strumia and Francesco Vissani. Precise quasielastic neutrino/nucleon cross-section. Physics Letters B, 564:42 - 54, 2003. 4.2.4

[130] NIST PSTAR. Database: http://physics.nist.gov/physrefdata/star/text/. 4.2 .4

[131] Basudeb Dasgupta and John F. Beacom. Reconstruction of supernova $\nu_{\mu}, \nu_{\tau}, \bar{\nu}_{\mu}$, and $\bar{\nu}_{\tau}$ neutrino spectra at scintillator detectors. Phys. Rev. D, 83:113006, 2011. 4.2.4, 5.1, 5.2

[132] F. Mantovani R. L. Rudnick Yu Huang, V. Chubakov and W. F. McDonough. A reference Earth model for the heat producing elements and associated geoneutrino flux. arXiv:1301.0365v2. 4.3

[133] Pietro Antonioli et. al. SNEWS: the Supernova Early Warning System. New Journal of Physics, 6:114, 2004. 4.3, 5.1 
[134] S V Bergh and G A Tammann. Galactic and extragalactic supernova rates. Annual Review of Astronomy and Astrophysics, 29:363-407, 1991. 5.1

[135] R. Diehl et. al. Nature (London), 439:45-47, 2006. 5.1

[136] M. Ikeda et. al. for The SuperKamiokande Collaboration. Search for supernova neutrino bursts at super-kamiokande. The Astrophysical Journal, 669:519, 2007. 5.1

[137] Abbasi, R. et. al. for the IceCube Collaboration. Icecube sensitivity for low-energy neutrinos from nearby supernovae. Astron. Astrophys., 535:A109, 2011. 5.1, 5.5

[138] Mathias Th. Keil, Georg G. Raffelt, and Hans-Thomas Janka. Monte carlo study of supernova neutrino spectra formation. The Astrophysical Journal, 590:971, 2003. 5.1

[139] Robert Buras, Hans-Thomas Janka, Mathias Th. Keil, Georg G. Raffelt, and Markus Rampp. Electron neutrino pair annihilation: A new source for muon and tau neutrinos in supernovae. The Astrophysical Journal, 587:320, 2003. 5.1

[140] Huaiyu Duan, George M. Fuller, and Yong-Zhong Qian. Collective neutrino flavor transformation in supernovae. Phys. Rev. D, 74:123004, 2006. 5.1

[141] K. Takahashi, K. Sato, H.E. Dalhed, and J.R. Wilson. Shock propagation and neutrino oscillation in supernova. Astroparticle Physics, 20:189193, 2003. 5.1

[142] Gianluigi Fogli, Eligio Lisi, Alessandro Mirizzi, and Daniele Montanino. Damping of supernova neutrino transitions in stochastic shock-wave density profiles. Journal of Cosmology and Astroparticle Physics, 2006:012, 2006. 5.1

[143] A. Friedland and A. Gruzinov. Neutrinos signatures of supernova turbulence. arXiv: astro-ph/0607244, 2006. 5.1

[144] Huaiyu Duan, George M. Fuller, and Yong-Zhong Qian. Collective Neutrino Oscillations. Ann.Rev.Nucl.Part.Sci., 60:569-594, 2010. 1 
[145] Sovan Chakraborty, Tobias Fischer, Alessandro Mirizzi, Ninetta Saviano, and Ricard Tomàs. No collective neutrino flavor conversions during the supernova accretion phase. Phys. Rev. Lett., 107:151101, 2011. 5.1, 5.3

[146] Srdjan Sarikas, Georg G. Raffelt, Lorenz Hüdepohl, and Hans-Thomas Janka. Suppression of self-induced flavor conversion in the supernova accretion phase. Phys. Rev. Lett., 108:061101, 2012. 5.1, 5.3

[147] John F. Beacom, Will M. Farr, and Petr Vogel. Detection of supernova neutrinos by neutrino-proton elastic scattering. Phys. Rev. D, 66:033001, 2002. 5.1

[148] Hisakazu Minakata, Hiroshi Nunokawa, Ricard Tomàs, and J.W.F. Valle. Probing supernova physics with neutrino oscillations. Physics Letters B, $542: 239-244,2002.5 .2$

[149] Solveig Skadhauge and Renata Zukanovich Funchal. Determining neutrino and supernova parameters with a galactic supernova. Journal of Cosmology and Astroparticle Physics, 2007:014, 2007. 5.2

[150] Abe, K. and et. al. Letter of Intent: The Hyper-Kamiokande Experiment Detector Design and Physics Potential. arXiv:1109.3262. 5.2

[151] Hisakazu Minakata, Hiroshi Nunokawa, Ricard Tomàs, and Jose W F Valle. Parameter degeneracy in flavor-dependent reconstruction of supernova neutrino fluxes. Journal of Cosmology and Astroparticle Physics, 2008:006, 2008. 5.2

[152] T. Fischer, S. C. Whitehouse, A. Mezzacappa, F.-K. Thielemann, and M. Liebendörfer. Protoneutron star evolution and the neutrino-driven wind in general relativistic neutrino radiation hydrodynamics simulations. Astronomy and Astrophysics, 517, 2010. 5.3

[153] L. Hüdepohl, B. Müller, H.-T. Janka, A. Marek, and G. G. Raffelt. Neutrino signal of electron-capture supernovae from core collapse to cooling. Phys. Rev. Lett., 104:251101, 2010. 5.3

[154] T. Fischer, G. Martínez-Pinedo, M. Hempel, and M. Liebendörfer. Neutrino spectra evolution during protoneutron star deleptonization. Phys. Rev. D, 85:083003, 2012. 5.3 
[155] C. Lunardini and A.Yu. Smirnov. Supernova neutrinos: Earth matter effects and neutrino mass spectrum. Nuclear Physics B, 616:307 - 348, 2001. 5.4

[156] A.S. Dighe, M. Kachelrieß, G.G. Raffelt, and R. Tomàs. Signatures of supernova neutrino oscillations in the Earth mantle and core. Journal of Cosmology and Astroparticle Physics, 2004:004, 2004. 5.4

[157] Adam M. Dziewonski and Don L. Anderson. Preliminary reference Earth model. Physics of the Earth and Planetary Interiors, 25:297 - 356, 1981. 5.4

[158] Evgeny Kh. Akhmedov, Michele Maltoni, and Alexei Yu. Smirnov. 1-3 leptonic mixing and the neutrino oscillograms of the earth. Journal of High Energy Physics, 2007(05):077, 2007. 5.4

[159] Evgeny Kh. Akhmedov, Michele Maltoni, and Alexei Yu. Smirnov. Neutrino oscillograms of the Earth: effects of 1-2 mixing and CPviolation. Journal of High Energy Physics, 2008(06):072, 2008. 5.4

[160] John F. Beacom and P. Vogel. Can a supernova be located by its neutrinos? Phys.Rev., D60:033007, 1999. 5.5

[161] Francis Halzen and Georg G. Raffelt. Reconstructing the supernova bounce time with neutrinos in icecube. Phys. Rev. D, 80:087301, 2009. 5.5

[162] K. Sumiyoshi, S. Yamada, H. Suzuki, and S. Chiba. Neutrino signals from the formation of a black hole: A probe of the equation of state of dense matter. Phys. Rev. Lett., 97:091101, 2006. 5.5

[163] Beacom, J. F., Boyd, R. N., and Mezzacappa, A. Black Hole Formation in Core-Collapse Supernovae and Time-of-Flight Measurements of the Neutrino Masses. Phys. Rev. D, 63:073011, 2001. 5.5 